\title{
Models of Care That Include Primary Care for Adult Survivors of Childhood Cancer: A Realist Review
}

Prepared for:

Agency for Healthcare Research and Quality

U.S. Department of Health and Human Services

5600 Fishers Lane

Rockville, MD 20857

www.ahrq.gov

Contract No. 75Q80120D00003

Prepared by:

The Johns Hopkins University Evidence-based Practice Center

Baltimore, MD

Investigators:

Claire Snyder, Ph.D.

Christina T. Yuan, Ph.D.

Renee F. Wilson, M.S.

Katherine Smith, Ph.D.

Youngjee Choi, M.D.

Paul C. Nathan, M.D., M.Sc.

Allen Zhang, B.S.

Karen A. Robinson, Ph.D.

AHRQ Publication No. 22-EHC003

February 2022 
This report is based on research conducted by the Johns Hopkins University Evidence-based Practice Center (EPC) under contract to the Agency for Healthcare Research and Quality (AHRQ), Rockville, MD (Contract No. 75Q80120D00003). The National Cancer Institute (NCI) of the National Institutes of Health (NIH) funded the report. The findings and conclusions in this document are those of the authors, who are responsible for its contents; the findings and conclusions do not necessarily represent the views of AHRQ or NCI/NIH. Therefore, no statement in this report should be construed as an official position of AHRQ, NCI/NIH, or the U.S. Department of Health and Human Services.

\section{None of the investigators have any affiliations or financial involvement that conflicts with the material presented in this report.}

The information in this report is intended to help healthcare decision makers - patients and clinicians, health system leaders, and policymakers, among others - make well-informed decisions and thereby improve the quality of healthcare services. This report is not intended to be a substitute for the application of clinical judgment. Anyone who makes decisions concerning the provision of clinical care should consider this report in the same way as any medical reference and in conjunction with all other pertinent information, i.e., in the context of available resources and circumstances presented by individual patients.

This report is made available to the public under the terms of a licensing agreement between the author and the Agency for Healthcare Research and Quality. This report may be used and reprinted without permission except those copyrighted materials that are clearly noted in the report. Further reproduction of those copyrighted materials is prohibited without the express permission of copyright holders.

AHRQ or U.S. Department of Health and Human Services endorsement of any derivative products that may be developed from this report, such as clinical practice guidelines, other quality enhancement tools, or reimbursement or coverage policies, may not be stated or implied.

AHRQ appreciates appropriate acknowledgment and citation of its work. Suggested language for acknowledgment: This work was based on an evidence report, Models of Care That Include Primary Care for Adult Survivors of Childhood Cancer: A Realist Review, by the Evidencebased Practice Center Program at the Agency for Healthcare Research and Quality (AHRQ).

Suggested citation: Snyder C, Yuan CT, Wilson RF, Smith K, Choi Y, Nathan PC, Zhang A, Robinson KA. Models of Care That Include Primary Care for Adult Survivors of Childhood Cancer: A Realist Review. Evidence Report. (Prepared by the Johns Hopkins University Evidence-based Practice Center under Contract No. 75Q80120D00003.) AHRQ Publication No. 22-EHC003. Rockville, MD: Agency for Healthcare Research and Quality; February 2022. DOI: 10.23970/AHRQEPCREALISTMODELSOFCARE. Posted final reports are located on the Effective Health Care Program search page. 


\section{Preface}

The Agency for Healthcare Research and Quality (AHRQ), through its Evidence-based Practice Centers (EPCs), sponsors the development of evidence reports and technology assessments to assist public- and private-sector organizations in their efforts to improve the quality of healthcare in the United States.

The reports and assessments provide organizations with comprehensive, evidence-based information on common medical conditions and new healthcare technologies and strategies. They also identify research gaps in the selected scientific area, identify methodological and scientific weaknesses, suggest research needs, and move the field forward through an unbiased, evidence-based assessment of the available literature. The EPCs systematically review the relevant scientific literature on topics assigned to them by AHRQ and conduct additional analyses when appropriate prior to developing their reports and assessments.

This report from the EPC Program at AHRQ is one of several efforts underway across the U.S. Department of Health and Human Services to implement provisions of the Childhood Cancer Survivorship, Treatment, Access, and Research (STAR) Act of 2018 (Public Law No: 115-180). The National Cancer Institute of the National Institutes of Health funded this report from the EPC Program at AHRQ. AHRQ assigned this report to the following EPC: The Johns Hopkins University Evidence-based Practice Center (Contract No. 75Q80120D00003).

To bring the broadest range of experts into the development of evidence reports and health technology assessments, AHRQ encourages the EPCs to form partnerships and enter into collaborations with other medical and research organizations. The EPCs work with these partner organizations to ensure that the evidence reports and technology assessments they produce will become building blocks for healthcare quality improvement projects throughout the Nation. The reports undergo peer review and public comment prior to their release as a final report.

AHRQ expects that the EPC evidence reports and technology assessments, when appropriate, will inform individual health plans, providers, and purchasers as well as the healthcare system as a whole by providing important information to help improve healthcare quality.

If you have comments on this evidence report, they may be sent by mail to the Task Order Officer named below at: Agency for Healthcare Research and Quality, 5600 Fishers Lane, Rockville, MD 20857, or by email to epc@ahrq.hhs.gov.

David Meyers, M.D.

Acting Director

Agency for Healthcare Research and Quality

Craig A. Umscheid, M.D., M.S.

Director

Evidence-based Practice Center Program

Center for Evidence and Practice Improvement

Agency for Healthcare Research and Quality
Arlene S. Bierman, M.D., M.S.

Director

Center for Evidence and Practice

Improvement

Agency for Healthcare Research and Quality

Suchitra Iyer, Ph.D.

Task Order Officer

Center for Evidence and Practice

Improvement

Agency for Healthcare Research and Quality 


\section{Acknowledgments}

The authors gratefully acknowledge Christine Chang, M.D, for providing guidance throughout the topic refinement and review process, and Jeanette Edelstein, M.A., for copy editing the draft report.

\section{Stakeholders}

During the evidence review the EPC consulted with Stakeholders who represent the end-users of research, as well as technical and content experts. The EPC sought Stakeholder input on the priority areas for research and synthesis. Broad expertise and perspectives were sought. Divergent and conflicting opinions are common and perceived as healthy scientific discourse that results in a thoughtful, relevant systematic review. Stakeholders are not involved in the analysis of the evidence or the writing of the report. Therefore, in the end, study questions, design, methodological approaches, and/or conclusions do not necessarily represent the views of individual Stakeholders.

Stakeholders must disclose any financial conflicts of interest greater than $\$ 5,000$ and any other relevant business or professional conflicts of interest. Because of their role as end-users or their unique clinical or content expertise, individuals with potential conflicts may be retained. The Task Order Officer (TOO) and the EPC work to balance, manage, or mitigate any conflicts of interest.

The list of Stakeholders who provided input to this report follows:

Anne Blaes, M.D.

University of Minnesota School of Medicine

Minneapolis, MN

Joanne Greenhalgh, Ph.D.*

University of Leeds

Leeds, UK

Melissa Hudson, M.D.*

St. Jude's Research Hospital

Memphis, TN

Shelley Fuld Nasso

National Coalition for Cancer Survivorship

Silver Spring, MD

Larissa Nekhlyudov, M.D., M.P.H.*

Brigham and Women's Hospital

Boston, MA

Kevin Oeffinger, M.D.*

Duke University School of Medicine

Durham, NC 
Carol Rosenberg, M.D.

University of Chicago Pritzker School of Medicine

Chicago, IL

Rosann Tucci, M.S., A.N.P.

Memorial Sloan Kettering Cancer Center

New York, NY

Susan Weiner, Ph.D.

Children's Cancer Cause

Washington, DC

*Provided input on Draft Report.

\section{Peer Reviewers}

Prior to publication of the final evidence report, EPCs sought input from independent Peer Reviewers without financial conflicts of interest. However, the conclusions and synthesis of the scientific literature presented in this report do not necessarily represent the views of individual reviewers. AHRQ may also seek comments from other Federal agencies when appropriate.

Peer Reviewers must disclose any financial conflicts of interest greater than $\$ 5,000$ and any other relevant business or professional conflicts of interest. Because of their unique clinical or content expertise, individuals with potential nonfinancial conflicts may be retained. The TOO and the EPC work to balance, manage, or mitigate any potential nonfinancial conflicts of interest identified.

The list of Peer Reviewers follows:

Linda Overholser, M.D., M.P.H.

University of Colorado, Anschutz Medical Campus

Denver, $\mathrm{CO}$

Mark Pearson, Ph.D.

Hull York Medical School

University of Hull

Hull, UK

Talya Salz, Ph.D.

Memorial Sloan Kettering Cancer Center

New York, NY 


\section{Models of Care That Include Primary Care for Adult Survivors of Childhood Cancer: A Realist Review}

\section{Structured Abstract}

Objectives. We had two aims: (1) identify and analyze models of survivorship care for adult survivors of childhood cancer that include primary care, and (2) identify available tools, training, and other resources for adult survivors of childhood cancer.

Methods. For each aim, we used realist synthesis to provide insights on how and for whom, in what contexts, and via what mechanisms the models of care and resources we identified can be effective for adult survivors of childhood cancer. We developed an initial program theory through searches of the literature and discussions with Stakeholders. We then identified and summarized quantitative evidence that supported or refuted the theory and developed specific hypotheses about how contexts and mechanisms may interact to produce outcomes (i.e., "CMO" hypotheses). The final program theory and CMO hypotheses were presented to Stakeholders for feedback.

Results. Our final refined theory describes how, within the overall environment, survivor and provider characteristics and facilitators/barriers interact to produce intermediate and final outcomes. We focus on the role of models of care and resources (e.g., care plans) in these interactions. The program theory variables seen most consistently in the literature include oncology care versus primary care, survivor and provider knowledge (i.e., survivor risks and needs), provider comfort treating childhood cancer survivors, communication and coordination between and among providers and survivors, and delivery/receipt of prevention and surveillance of late effects of original cancer treatment. In turn, these variables played the most prominent role in the seven CMO hypotheses ( 4 focused on survivors and 3 focused on providers) regarding what works for whom and in what circumstances.

Conclusions. To enable models of care that include primary care for adult survivors of childhood cancer, there needs to be communication of knowledge to both survivors and primary care providers. Our program theory provides guidance on the ways this knowledge could be shared, including the role of resources in doing so, and our CMO hypotheses suggest how the relationships illustrated in our theory could be associated with survivors living longer and feeling better through high-value care. 


\section{Contents}

Evidence Summary ..................................................................................................................................... 1

Introduction ................................................................................................................................ 1

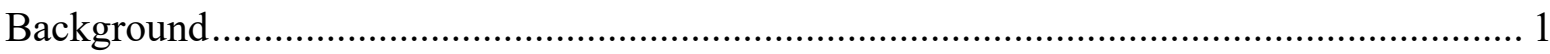

Purpose and Scope of the Review ............................................................................. 1

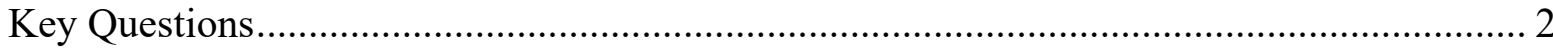

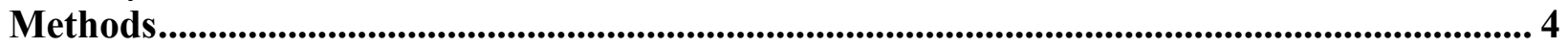

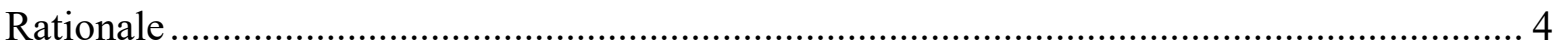

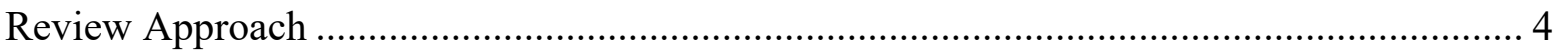

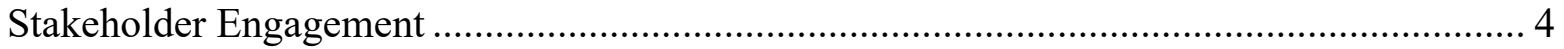

Development of Initial Program Theory ............................................................................ 5

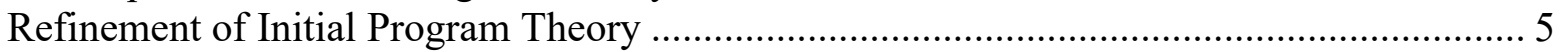

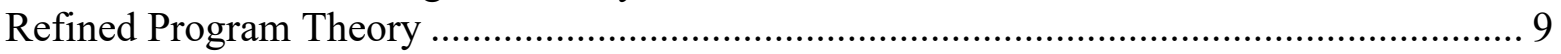

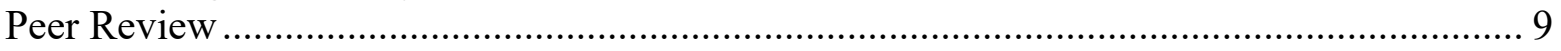

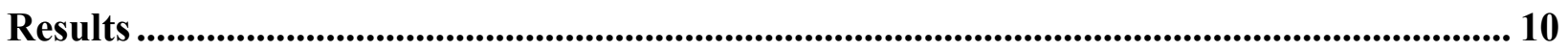

Results of Searching ………………........................................................................ 10

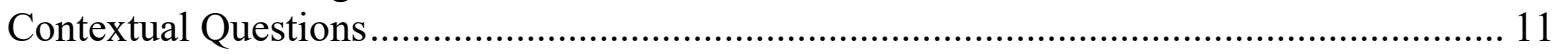

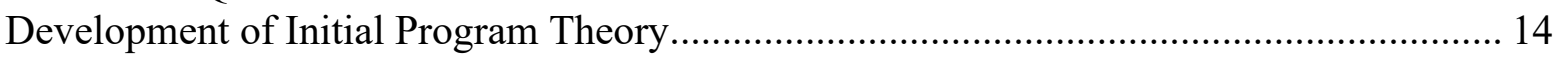

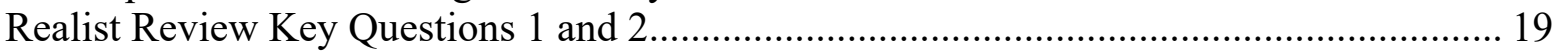

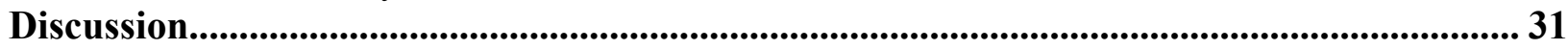

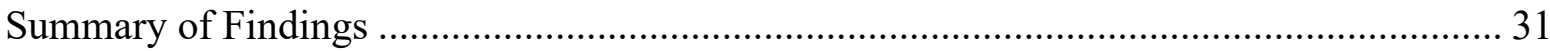

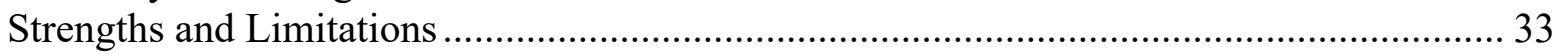

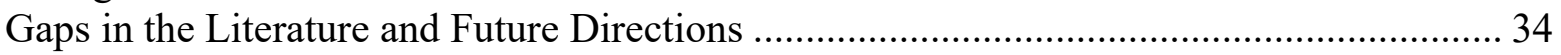

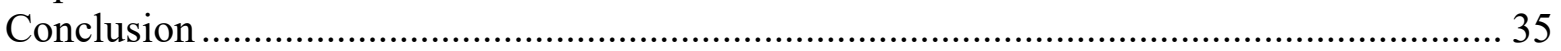

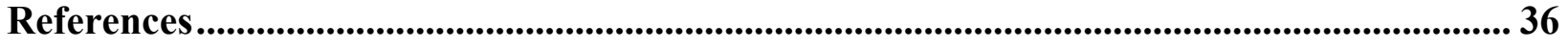

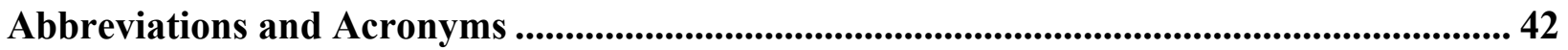

\section{Tables}

Table A. CMO hypotheses regarding how models of care that include primary care could be effective, focusing on the survivor intermediate outcome.......................................................... Table B. CMO hypotheses regarding how models of care that include primary care could be effective, focusing on the primary care provider intermediate outcome .....................................

Table 1. Eight models of cancer survivorship care as described by ASCO. ................................12

Table 2. CMO hypotheses regarding how models of care that include primary care could be

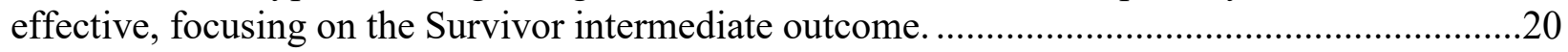

Table 3. CMO hypotheses regarding how models of care that include primary care could be effective, focusing on the primary care provider intermediate outcome .....................................25

\section{Figures}

Figure A. Refined Program Theory .....

Figure 1. Steps from development of the Initial Program Theory to Refined Program Theory......6

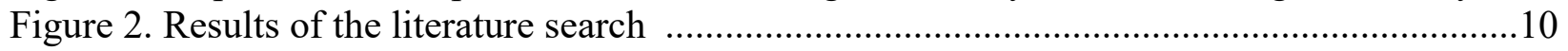

Figure 3. Refined Program Theory ……………………............................................................ 17

\section{Appendixes}

Appendix A. Search Strategies

Appendix B. Example Data Extraction Table Content 
Appendix C. Summary of Data Abstraction

Appendix D. List of References Used To Inform the Contextual Questions and Initial Program Theory

Appendix E. List of Survivorship Resources Available to Cancer Survivors, Their Families, and Their Medical Care Providers

Appendix F. Program Theory Variable List

Appendix G. Initial Program Theory 


\section{Evidence Summary}

\section{Main Points}

- Our program theory describes how survivor and provider characteristics and facilitators/barriers may interact to produce intermediate and final outcomes and the potential role of models and resources in these interactions.

- The program theory variables seen most consistently in the literature include oncology care versus primary care, survivor and provider knowledge (e.g., survivor risks and needs), provider comfort treating childhood cancer survivors, communication and coordination between and among providers and survivors, and delivery/receipt of prevention and surveillance of late effects of original cancer treatment.

- We developed seven hypotheses about the relationships between context, mechanism, and outcomes (CMO) that could be associated with effective survivorship care models that include primary care.

- Care delivered outside of the specialty setting needs to include communication of knowledge to both survivors and primary care providers; our program theory provides guidance on the ways this knowledge could be shared.

\section{Background and Purpose}

Childhood cancer survivors are at increased risk for life-long chronic morbidities owing to their cancer and its treatment. There is no practical taxonomy for survivorship models of care. There is also a lack of clarity about which models are appropriate for whom and in what circumstances, as well as how resources (e.g., education, support groups, care plans, and care management processes) can support quality survivorship care.

The key decisional dilemmas for providers, survivors, caregivers, and health systems are (1) what models of childhood cancer survivorship care that include primary care may improve shortand long-term outcomes, for which survivors, and under what circumstances, and (2) what tools, trainings, processes, and other resources can promote quality survivorship care across the various models?

\section{Methods}

We addressed Contextual Questions about the different types of models of care and resources through review of literature and discussions with Stakeholders. For the purposes of this project, the term "resources" includes long-term followup guidelines; educational materials; trainings; survivor care documents (e.g., survivorship care plans); survivorship care management processes (e.g., expedited routes of contact for consultation); and survivor supportive tools and services (e.g., support groups).

We addressed the decisional dilemmas by conducting a realist review. We interviewed Stakeholders (survivor advocates, providers, researchers) and conducted targeted and iterative searching to (1) identify models of survivorship care and analyze the program theories (underlying ideas and assumptions) about how they are intended to work; and (2) identify available tools, training, and other resources for childhood cancer survivorship care and analyze the program theories underlying how they are intended to be implemented. Finally, we refined 
the identified program theory and developed hypotheses about context, mechanism, and outcomes through review of quantitative evidence and discussions with Stakeholders.

\section{Results}

There are many models of survivorship care with no practical taxonomy. We identified four differentiating factors across models: (1) the inclusion of survivorship expertise (whether via a specialized primary care provider or oncologist; or a physician, nurse practitioner/physician assistant, or multidisciplinary team); (2) the role of the primary care provider (e.g., main provider of survivorship care, provides survivorship care under the guidance of survivorship expert, provides primary care with no particular attention to survivorship); (3) degree of access to academic/cancer center support for survivors and/or providers; and (4) delivery of consultative versus longitudinal care. In practice, what is seen in the literature (and experienced by survivors) are more often patterns of care that occur not by design but owing to circumstance. We identified 40 resources freely available to both survivors and providers: 23 survivor-specific, 12 provider/researcher-specific, 5 for both. Discussions with Stakeholders suggested that resources are most helpful if they are easy to access, user-friendly, known to survivors and providers, from trustworthy sources, and valued by survivors and their families.

At the most basic level, models of care that include primary care need to ensure that survivors and providers have the necessary information to obtain/deliver appropriate care, and resources are a key mechanism in providing this information. The program theory variables seen most consistently in the literature include oncology care versus primary care, survivor and provider knowledge (i.e., survivor risks and needs), provider comfort treating childhood cancer survivors, communication and coordination between and among providers and survivors, and delivery/receipt of prevention and surveillance of late effects of the original cancer treatment. In turn, these variables played the most prominent role in hypothesizing what works for whom and in what circumstances. The provider health practices and survivor health services use/behaviors represent the intermediate outcomes for survivors and providers as described by the Stakeholders: "Survivors feel confident about sharing their history, know their risks, recognize symptoms and problems, understand the care they need, are aware of the resources available to help them, and can access relevant care and services" and "PCPs understand a survivor's history, know the survivor's risks, recognize symptoms and problems, understand the care survivors need, are aware of the resources available to help them, and can access relevant care and services." Our program theory illustrates how, within an environment, survivor and provider characteristics and facilitators/barriers, may connect through models of care and resources to achieve these intermediate outcomes for survivors and providers, ultimately leading to survivors living longer and feeling better (Figure A).

We developed seven hypotheses about the relationships of context, mechanisms, and outcomes (Tables A and B) for how models of care that include primary care, and resources, could be effective in providing care to adult survivors of childhood cancer.

We hypothesized that four mechanisms would be associated with higher levels of the survivor intermediate outcome:

1. Linking resources with information that survivors can themselves use and also share with their primary care provider (PCP),

2. Identifying perceived/actually healthier survivors who have perceived/actually lower needs for survivorship-specific care, 
3. Connecting survivors' engagement in the health system with increased knowledge about survivorship care, and

4. Suggesting that survivors with greater confidence in their PCPs would be more willing to transition their care.

We hypothesized that three mechanisms would be associated with higher levels of the provider intermediate outcome:

1. Linking resources with information to guide the PCP in delivering survivorship care,

2. Identifying the shared care model as a way to obtain the needed support from oncologists, and

3. Suggesting that PCPs with more experience caring for childhood cancer survivors would have greater comfort and expertise in doing so.

\section{Limitations}

Evidence on adult survivors of childhood cancer was limited leading us to consider studies of adult survivors of adult cancer. There was a lack of formal evaluations of models of care and data on final outcomes, particularly mortality, are sparse. A challenge was conducting a realist review of multiple ill-defined patterns of care rather than one intervention or model of care. Our review was also limited by the relatively short amount of time available, precluding the depth and number of iterative searches, syntheses, and refinement of theory typical in a realist review.

\section{Implications and Conclusions}

If care for adult survivors of childhood cancer is to be delivered outside of the specialty setting, there needs to be communication of knowledge about survivorship care to both survivors and primary care providers. Our program theory provides guidance on the ways this knowledge could be shared. Our context, mechanism, and outcome hypotheses suggest how the relationships illustrated in our theory could be associated with survivors living longer and feeling better through high-value care.

Future research is needed to address two essential research questions related to this review: (1) who needs to be seen in specialty care and who can be followed in their own community; and (2) for those followed in the community, how can the knowledge that survivors and PCPs need to receive/deliver quality care be effectively transferred? 
Figure A. Refined program theory

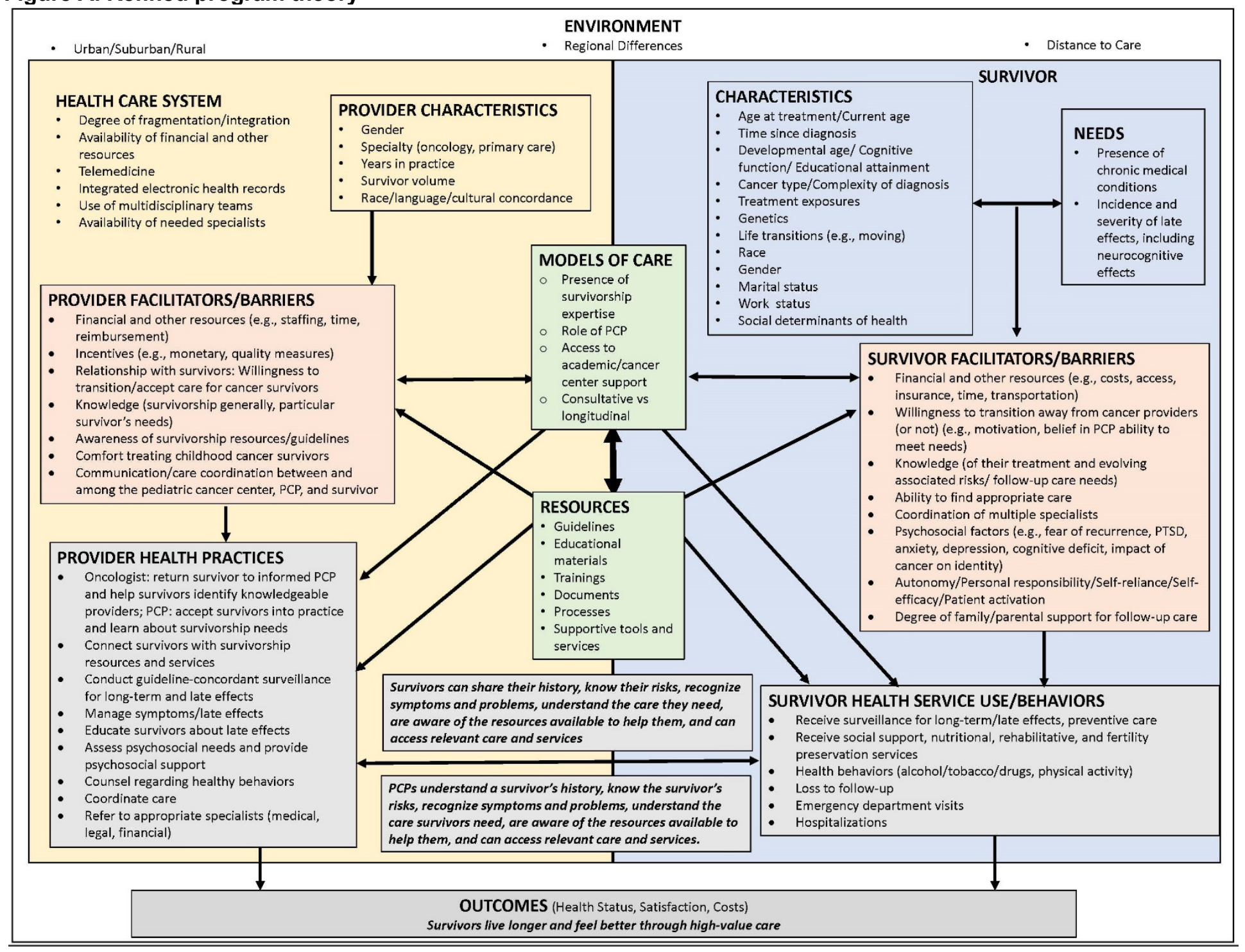

$\mathrm{PCP}=$ primary care provider; $\mathrm{PTSD}=$ post-traumatic stress disorder 
Table A. Context-mechanism-outcome hypotheses regarding how models of care that include primary care could be effective, focusing on intermediate outcomes for the survivor

\begin{tabular}{|c|c|c|c|}
\hline In the CONTEXT of & This MECHANISM ... Produces & $\begin{array}{l}\text { OUTCOME } \\
\text { (Intermediate) }\end{array}$ & $\begin{array}{l}\text { OUTCOME } \\
\text { (Final) }\end{array}$ \\
\hline $\begin{array}{l}\text { A) the availability of } \\
\text { survivorship care plans, } \\
\text { guidelines, and other } \\
\text { resources }\end{array}$ & $\begin{array}{l}\text { A1) improved survivor knowledge } \\
\text { A2) information available to } \\
\text { share with PCP to inform delivery } \\
\text { of survivorship-related care }\end{array}$ & $\begin{array}{l}\text { Survivors can share } \\
\text { their history, know their } \\
\text { risks, recognize } \\
\text { symptoms and } \\
\text { problems, understand } \\
\text { the care they need, are } \\
\text { aware of the resources } \\
\text { available to help them, } \\
\text { and can access relevant } \\
\text { care and services. }\end{array}$ & $\begin{array}{l}\text { Survivors live } \\
\text { longer and feel } \\
\text { better through } \\
\text { high-value care. }\end{array}$ \\
\hline $\begin{array}{l}\text { B) healthier survivors } \\
\text { (perceived or actual) }\end{array}$ & $\begin{array}{l}\text { B) less perceived/actual need for } \\
\text { survivorship-related care }\end{array}$ & $\begin{array}{l}\text { Survivors can share } \\
\text { their history, know their } \\
\text { risks, recognize } \\
\text { symptoms and } \\
\text { problems, understand } \\
\text { the care they need, are } \\
\text { aware of the resources } \\
\text { available to help them, } \\
\text { and can access relevant } \\
\text { care and services. }\end{array}$ & $\begin{array}{l}\text { Survivors live } \\
\text { longer and feel } \\
\text { better through } \\
\text { high-value care. }\end{array}$ \\
\hline $\begin{array}{l}\text { C) survivors engaged in } \\
\text { healthcare system }\end{array}$ & C) improved knowledge & $\begin{array}{l}\text { Survivors can share } \\
\text { their history, know their } \\
\text { risks, recognize } \\
\text { symptoms and } \\
\text { problems, understand } \\
\text { the care they need, are } \\
\text { aware of the resources } \\
\text { available to help them, } \\
\text { and can access relevant } \\
\text { care and services. }\end{array}$ & $\begin{array}{l}\text { Survivors live } \\
\text { longer and feel } \\
\text { better through } \\
\text { high-value care. }\end{array}$ \\
\hline $\begin{array}{l}\text { D) survivor confidence in } \\
\text { PCPs }\end{array}$ & D) willingness to transition care & $\begin{array}{l}\text { Survivors can share } \\
\text { their history, know their } \\
\text { risks, recognize } \\
\text { symptoms and } \\
\text { problems, understand } \\
\text { the care they need, are } \\
\text { aware of the resources } \\
\text { available to help them, } \\
\text { and can access relevant } \\
\text { care and services. }\end{array}$ & $\begin{array}{l}\text { Survivors live } \\
\text { longer and feel } \\
\text { better through } \\
\text { high-value care. }\end{array}$ \\
\hline
\end{tabular}

$\mathrm{PCP}=$ primary care provider 
Table B. Context-mechanism-outcome hypotheses regarding how models of care that include primary care could be effective, focusing on intermediate outcomes for the primary care provider

\begin{tabular}{|c|c|c|c|}
\hline In the CONTEXT of & $\begin{array}{l}\text { This MECHANISM ... } \\
\text { Produces }\end{array}$ & $\begin{array}{l}\text { OUTCOME } \\
\text { (Intermediate) }\end{array}$ & $\begin{array}{l}\text { OUTCOME } \\
\text { (Final) }\end{array}$ \\
\hline $\begin{array}{l}\text { A) the availability of } \\
\text { survivorship care plans, } \\
\text { guidelines, and other } \\
\text { resources }\end{array}$ & $\begin{array}{l}\text { A) information available to } \\
\text { guide the PCP in delivering } \\
\text { survivorship-related care }\end{array}$ & $\begin{array}{l}\text { PCPs understand a } \\
\text { survivor's history, know } \\
\text { the survivor's risks, } \\
\text { recognize symptoms } \\
\text { and problems, } \\
\text { understand the care } \\
\text { survivors need, are } \\
\text { aware of the resources } \\
\text { available to help them, } \\
\text { and can access relevant } \\
\text { care and services. }\end{array}$ & $\begin{array}{l}\text { Survivors live } \\
\text { longer and feel } \\
\text { better through high- } \\
\text { value care. }\end{array}$ \\
\hline $\begin{array}{l}\text { B) PCP shared-care with } \\
\text { oncologist }\end{array}$ & $\begin{array}{l}\text { B) support from the } \\
\text { oncologist to aid the PCP in } \\
\text { delivering survivorship-related } \\
\text { care }\end{array}$ & $\begin{array}{l}\text { PCPs understand a } \\
\text { survivor's history, know } \\
\text { the survivor's risks, } \\
\text { recognize symptoms } \\
\text { and problems, } \\
\text { understand the care } \\
\text { survivors need, are } \\
\text { aware of the resources } \\
\text { available to help them, } \\
\text { and can access relevant } \\
\text { care and services. }\end{array}$ & $\begin{array}{l}\text { Survivors live } \\
\text { longer and feel } \\
\text { better through high- } \\
\text { value care. }\end{array}$ \\
\hline $\begin{array}{l}\text { C) more experience } \\
\text { caring for childhood } \\
\text { cancer survivors }\end{array}$ & $\begin{array}{l}\text { C) greater comfort caring for } \\
\text { childhood care survivors }\end{array}$ & $\begin{array}{l}\text { PCPs understand a } \\
\text { survivor's history, know } \\
\text { the survivor's risks, } \\
\text { recognize symptoms } \\
\text { and problems, } \\
\text { understand the care } \\
\text { survivors need, are } \\
\text { aware of the resources } \\
\text { available to help them, } \\
\text { and can access relevant } \\
\text { care and services. }\end{array}$ & $\begin{array}{l}\text { Survivors live } \\
\text { longer and feel } \\
\text { better through high- } \\
\text { value care. }\end{array}$ \\
\hline
\end{tabular}

$\mathrm{PCP}=$ primary care provider 


\section{Introduction}

\section{Background}

The American Cancer Society estimates that there were over 110,000 cancer survivors ages 0-19 as of January 1, 2019; when added to adult survivors of childhood (ages 0-14) and adolescent (ages 15-19) cancer, the total number of survivors approaches 400,000. ${ }^{1}$ The growth in the number of survivors reflects new and more effective therapies, better risk stratification, and progress in supportive care that have improved treatment outcomes over the past decades, with 5-year relative survival increasing from 58 percent (1975-1977) to 84 percent (2008-2014) for children and from 68 percent (1975-1977) to 85 percent (2008-2014) for adolescents with cancer. ${ }^{1}$ Unfortunately, owing to their cancer and its treatment, the majority of adult survivors experience life-long, chronic morbidities, such as cardiomyopathy, metabolic syndrome, and subsequent malignant neoplasms. ${ }^{2-6}$ However, the impacts of cancer and its treatment are not uniform, with different risks related to the specific type and location of previous cancer, its therapy, genetic predispositions, lifestyle behaviors, and comorbid health conditions. ${ }^{7}$ Thus, the National Academy of Medicine (NAM; formerly the Institute of Medicine) recommends lifelong followup based on these factors. ${ }^{8}$

There is a lack of clarity regarding the appropriate models of survivorship care. Models of care range from specialized survivorship followup to general oncology followup to primary care followup. There are multiple barriers that preclude many childhood cancer survivors from receiving specialized long-term followup care, but there are also barriers that preclude those survivors from receiving quality survivorship care in primary care. ${ }^{9,10}$

\section{Purpose and Scope of the Review}

Given the growing number of childhood cancer survivors (those diagnosed before age 21), the extensive morbidity and mortality experienced by these survivors, the need for risk-based survivorship care, and the lack of clarity regarding the appropriate models of childhood survivorship care, the key decisional dilemmas are (1) what models of childhood cancer survivorship care improve short- and long-term outcomes, for which survivors, and under what circumstances and (2) what tools, trainings, processes, and other resources can promote quality survivorship care across the various models? The National Cancer Institute (NCI) requested a realist review as part of a series of projects for The Childhood Cancer Survivorship, Treatment, Access, and Research (STAR) Act to better understand the state of the science and ultimately improve the care and quality of life for childhood and adolescent cancer survivors. This and the other requested reports are intended to inform decision making regarding NCI's future investments in research.

We conducted a realist review to address two aims: (1) to identify and analyze models of survivorship care for adult survivors of childhood cancer that include primary care using realist synthesis and (2) to identify available tools, training, and other survivorship resources for adult survivors of childhood cancer and use realist synthesis to understand how and why they produce effects on health outcomes.

A realist review differs from a systematic review in multiple ways. For example, a realist review does not summarize existing evidence of whether an intervention works, but investigates theories regarding how interventions are intended to work and how different contexts affect the mechanisms by which they work to achieve the intended outcomes. Also, whereas systematic 
reviews have strict inclusion/exclusion criteria on studies to review and synthesize, realist reviews may draw from literature ranging from studies directly applicable to the topic to studies on related topics in different disease areas or populations. Further, the outputs of systematic reviews are syntheses of the evidence, whereas the outputs of realist reviews are theories and hypotheses regarding how interventions are intended to work.

The aim of this realist review was to address the question of how models of care that include primary care, and related resources, could be effective - not whether they are effective. The review focused specifically on survivorship care, not the overall care of adult survivors of childhood cancer (e.g., addressing unrelated comorbid conditions, general preventive care). The review was not intended to explore whether models of survivorship care that include primary care are more or less effective than models of care that do not include primary care, but to describe for whom and in what circumstances models of survivorship care that include primary care could be effective.

For the purposes of this review, we included survivors of cancer diagnosed before age 21; for simplicity, we do not distinguish between childhood and adolescent survivors and refer to childhood cancer survivors throughout. Our review focused on survivorship care, not cancer treatment. We included studies examining care by physicians, nurse practitioners, and physician assistants, and used whatever definitions of primary care were applied in the included studies.

\section{Key Questions}

\section{Contextual Questions (CQs)}

CQ1. How is effectiveness defined and measured for survivorship care models for adult survivors of childhood cancer (cancer diagnosed prior to age 21 years old)?

CQ2. What are the models of survivorship care for adult survivors of childhood cancer?

a. Which of these models include primary care?

i. What is the evidence of effectiveness of the different models that include primary care?

CQ3. What survivorship care resources are available for adult survivors of childhood cancer and their families?

a. What are the intended outcomes of the different resources available for adult survivors of childhood cancer and their families?

b. What is the evidence of effectiveness of the different resources available for adult survivors of childhood cancer and their families?

c. What are the monetary costs to access these resources?

CQ4. What survivorship care resources are available to providers who care for adult survivors of childhood cancer?

a. What are the intended outcomes of the different resources available to care providers? 

b. What is the evidence of effectiveness of the different resources available to care providers?
c. What are the monetary costs to access these resources?

\section{Key Questions (KQs) for the Realist Review}

KQ1. For whom and under what circumstances could different survivorship care models for adult survivors of childhood cancer (cancer diagnosed prior to age 21 years old) that include primary care be effective?

a. What are the key mechanisms by which these models could be effective?

b. What are important contexts that determine whether different mechanisms could be effective?

KQ2. For whom and under what circumstances could different survivorship care resources for adult survivors of childhood cancer be effective in achieving their intended outcomes?
a. For survivors and their families
i. What are the key mechanisms by which these resources could lead to their intended outcome?
ii. What are important contexts that determine whether different mechanisms could lead to outcomes?
b. For care providers
i. What are the key mechanisms by which these resources could lead to their intended outcome?
ii. What are important contexts that determine whether different mechanisms could lead to outcomes?

For the purposes of this project, the term "resources" includes-

- Long-term followup guidelines;

- Educational materials directed at either survivor/family or care providers, regardless of media (i.e., electronic, hard copy);

- In-person or virtual trainings (i.e., workshops, conferences, continuing medical education courses) directed at either survivor/family or care providers;

- Survivor care documents (i.e., survivor-specific standardized letters, treatment summaries, survivorship care plans);

- Survivorship care management processes (i.e., expedited routes of contact for consultation, re-referral, support services; methods for digitizing and securely distributing health records; and other provider-to-provider and provider-to-survivor communications); and

- Survivor supportive tools and services (in-person or digital), such as text messaging/peer navigator programs, support groups (in-person, telephone-based, or online), and professional psychosocial counselors (in-person, telephone-based, or online). 


\section{Methods}

\section{Rationale}

A realist synthesis or review addresses the question, "What works, how, why, for whom, to what extent and in what circumstances, in what respect and over what duration?" 11 In a realist review, the underlying ideas and assumptions about how an intervention works are first identified and expressed in an initial program theory. The initial program theory is then tested and refined.

Realist synthesis also seeks to describe context-mechanism-outcome (CMO) hypotheses around this program theory. These $\mathrm{CMO}$ hypotheses describe how contextual features $(\mathrm{C})$ can influence mechanisms $(\mathrm{M})$ through which an intervention or strategy are purported to work to produce outcomes $(\mathrm{O})$. These CMO hypotheses explore factors such as the way the intervention is designed, how it is implemented and in what settings, and the different characteristics of recipients. To refine the initial program theory, we sought and synthesized quantitative evidence to identify how different contexts trigger different mechanisms that result in different intended and unintended outcomes (CMO hypotheses).

In this realist review, we first identified models of survivorship care and available tools, training, and other resources for childhood cancer survivorship care. We then developed an initial program theory about how these models of care and resources were intended to achieve intermediate and final outcomes. We tested and refined the initial program theory by reviewing quantitative evidence evaluating the use of models and resources in practice. We also developed CMO hypotheses to explain how different contexts shape the mechanisms through which the models of care and resources work.

\section{Review Approach}

Questions were initially identified by the National Cancer Institute (NCI) and the Agency for Healthcare Research and Quality (AHRQ) and focused through a topic refinement process. We organized the questions as Contextual Questions (CQs) and realist review questions [or Key Questions, (KQs)]. Both the CQs and KQs were addressed through interviews with Stakeholders and literature review. The answers to the CQs describe the dimensions of models of care and resources for adult survivors of childhood cancer and their families and providers. The theory and $\mathrm{CMO}$ development process address the KQs by describing how models of care that include primary care and relevant resources could be effective. In practice, the theory and CMO hypotheses are the "answers" to the KQs.

Our protocol was posted for public comment by AHRQ, and we have followed the Realist And Meta-narrative Evidence Syntheses: Evolving Standards (RAMESES) guidelines in reporting our review. ${ }^{12}$

\section{Stakeholder Engagement}

We identified nine Stakeholders with a diverse range of perspectives, including clinicians with expertise in cancer survivorship and survivorship care, clinicians with expertise in cancer survivorship research, and childhood cancer survivorship patient advocates and caregivers. We convened small-group meetings of the Stakeholders at three stages throughout the project to help clarify the relevant concepts related to models of care and resources (at theory development); to present an initial program theory and elicit feedback; and to elicit feedback on the refined 
program theory and $\mathrm{CMO}$ hypotheses. We engaged a realist synthesis expert throughout the project, as needed, to provide guidance on realist methods.

\section{Development of Initial Program Theory}

As with all realist reviews, our methods were iterative and included multiple searches of the literature and consultations with the Stakeholders. The objective was to develop an initial program theory and then refine it as our understanding grew based on the literature and our Stakeholders" input. For clarity, we use the term "model" to refer to "model of care" and "theory" to refer to program theory and existing theories.

We developed an initial program theory by engaging Stakeholders, considering existing theories applicable to the topic, and reviewing literature that described expectations for models of care and resources. ${ }^{13,14}$ This stage of our review focused on conceptual and "thought" pieces regarding how models of care and resources are intended to work, for whom, and in what contexts. During this stage we sought evidence from a range of sources (Figure 1, Steps 1 and 2):

- Information from the team regarding their knowledge of mid-range theories related to access to care, knowledge specialization, coordination across the continuum of care, and uptake and use of resources.

- Opinion pieces, editorials, commentaries, and qualitative and mixed-methods studies about how models of care and resources are intended to work.

- Stakeholders' input on the initial program theory regarding how it did (or did not) reflect their experience and understanding of the issues, and on additional theories that may be useful for integration. ${ }^{15}$

\section{Refinement of Initial Program Theory}

\section{Selection of Documents}

We conducted additional searches to refine our program theory. In these searches we sought quantitative evidence that supported or refuted our initial program theory. Where applicable, we also incorporated the quantitative findings from mixed-methods studies used in the initial program theory development (Figure 1, Step 3).

We identified relevant quantitative studies by:

- Searching three databases (PubMed, CINAHL, PsycInfo),

- Posting a notice seeking information in the federal register, ${ }^{16}$

- Conducting a general search of Google Scholar to identify documents relevant to 'childhood cancer' (or related terms) and survivorship,

- Using snowballing (searching references of studies) and berry picking (finding information bit by bit using a range of sources including expert input) to identify additional studies. ${ }^{15,17,18}$

The initial program theory informed search terms for the databases. We did not limit our searches by study design or location of study. See Appendix A for the search strategies. 
Figure 1. Steps from development of the initial program theory to refined program theory

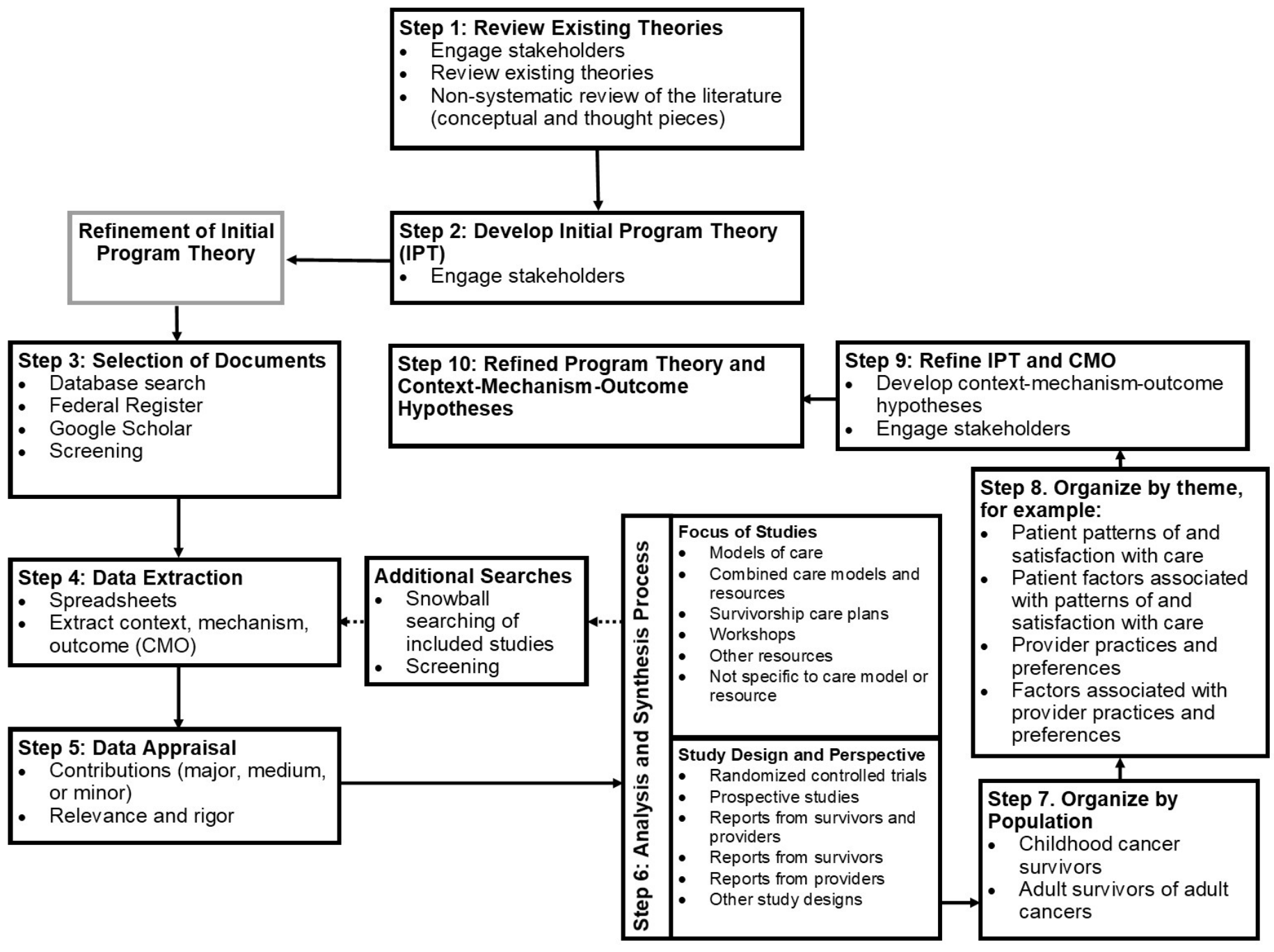


To screen abstracts and full-text articles, we followed methods described by Carrieri. ${ }^{19}$ Each abstract was screened by one of the team members. A random sample of the screened abstracts was checked by another team member for consistency.

\section{Data Extraction and Appraisal}

For program theory refinement, we focused on extracting evidence to support or refine fragments of the initial program theory or specific CMO hypotheses (Appendix B). We extracted study design and purpose; population of interest; and specific cohort, if applicable; as well as details on the models and /or resources under investigation. We identified the study variables, as defined in the initial program theory; variables with associations; and any other findings as they related to the initial program theory. We also drew from study authors' impressions to inform possible CMO associations. Data extraction for each study was completed by one team member (Figure 1, Steps 4 and 5).

Included studies were classified as those making major, medium, or minor contributions in informing and testing the program theory. ${ }^{19}$ Our criteria for classifying studies were adapted from the categorization criteria from Carrieri et al., 2018: ${ }^{19}$

- Major: Evaluates a model of care or resource for adult survivors of childhood cancer

- Medium:

- Provides insights regarding CMO connections in adult survivors of childhood or adult cancer and/or

- Describes, but does not evaluate, a model of care or resource

- Minor: Conducted in non-cancer contexts but mechanisms could plausibly operate in the context of childhood cancer survivorship

Notably, we found so few studies classified as "major" that, in practice, we prioritized our evaluation of the literature by primarily drawing from the literature focused on childhood cancer survivors (both during childhood/adolescence/young adulthood and adulthood) and supplementing with evidence from adult survivors of adult cancers where relevant and informative.

Studies were also assessed as to whether (1) the data were relevant to the initial program theory (relevance) and (2) the research methods supported the conclusions (rigor).

Considerations of rigor were informed by a set of questions derived from the Critical Appraisal Skills Programme (CASP) Checklist for Qualitative Research. ${ }^{20}$

The following questions guided consideration of rigor-

- Are the data credible and trustworthy?

- Are the methods appropriate to address the research goal?

- Was the research design justified?

- Was the data collected to address the research issue?

The following questions guided consideration of relevance-

- Is the study population specific to adult survivors of childhood cancer?

- Is the setting within or outside of the U.S.?

- Are the findings generalizable to our initial program theory?

Rather than being used to determine inclusion or exclusion, these considerations informed how we interpreted the findings and incorporated them into our thinking about CMO hypotheses. For example, we included studies of adult survivors of adult cancers, but we gave more weight to 
studies in our target population. For the question of generalizability to our initial program theory, we considered the extent to which the study addressed the variables in our initial program theory and how they relate to one another.

\section{Analysis and Synthesis Process}

We reviewed data extractions during team meetings, first based on the spreadsheet (Appendix B) and later using two sets of summary documents. The first set of documents summarized findings by study ("Study" documents). The second set of documents summarized findings by theme ("Theme" documents).

The first version of the Study document (Appendix C) was organized by study and included the study identification number, design, population (including sample size), and setting. Then, for each study, the document summarized the findings from the study related to the initial program theory, specifying how variables from the initial program theory were evaluated.

In addition to summarizing the study findings with labels of relevant initial program theory variables, the Study document also included in italics, speculations by the abstractor regarding how the findings related to potential CMO hypotheses (Figure 1, Step 6-9).

The first version of the Study document organized the studies by focus:

- Models of care,

- Combined care models and resources,

- Survivorship care plans,

- Workshops,

- Other resources, and

- Not specific to care model or resource

and study design and perspective:

- Randomized controlled trials,

- Prospective studies,

- Reports from survivors and providers,

- Reports from survivors,

- Reports from providers, and

- Other study design.

The second version of the Study document was organized with all studies related to childhood cancer survivors first, followed by studies from other populations (Appendix C). It was this version of the document that was primarily used by the team to develop the CMO hypotheses.

We then created a Theme document that organized the findings across studies by topic. For example, under Models of Care, we grouped findings related to the following topics:

- Patient patterns of and satisfaction with care,

- Patient factors associated with patterns of and satisfaction with care,

- Provider practices and preferences,

- Factors associated with provider practices and preferences. 
In the second version of the Theme document, we "boiled down" the topics into relevant themes, such as "who gets care where," "what care is received," and "provider preference and comfort." In the third version of the Theme document, we documented impressions based on the findings. For example, for the theme of "who gets care where," we noted the lack of data regarding who is being seen where and that there is no consistency in how this is measured or reported. We then developed overall themes across topics, such as "childhood cancer survivors and primary care providers want oncologists involved in care, though healthier childhood cancer survivors might be different in this regard." The final version of the Theme document added relevant findings from the literature regarding adult survivors of adult cancers, using the second version of the Study document.

Through this engagement with the study findings in the various iterations of the Study and Theme documents, the team discussed the implications of our findings for (1) refining the program theory, (2) updating the list of variables in the program theory, and (3) developing the CMO hypotheses. ${ }^{19}$

\section{Refined Program Theory}

We conducted a final meeting with our Stakeholders during which we presented our revised program theory, including updates to the list of variables, and proposed CMO hypotheses. We elicited feedback regarding whether our findings reflected our Stakeholders' understanding and experience. Based on this feedback, we made final revisions to the refined program theory, variables, and CMO hypotheses (Figure 1, Step 10).

\section{Peer Review}

Experts in the fields of cancer survivorship, primary care, oncology, and realist review methodology, as well as caregivers, were invited to provide external peer review of the KQs and protocol prior to the review. AHRQ and representatives from the NCI also provided comments. The draft report was sent to peer reviewers and the Stakeholders, while simultaneously posted on the AHRQ website for 4 weeks for public comment. 


\section{Results}

\section{Results of Searching}

The results of the literature searches are detailed in Figure 2. We identified 899 potentially relevant documents in the iterative search for program theory refinement. Of these, 135 met inclusion criteria and were included in the final review.

\section{Figure 2. Results of the literature search}

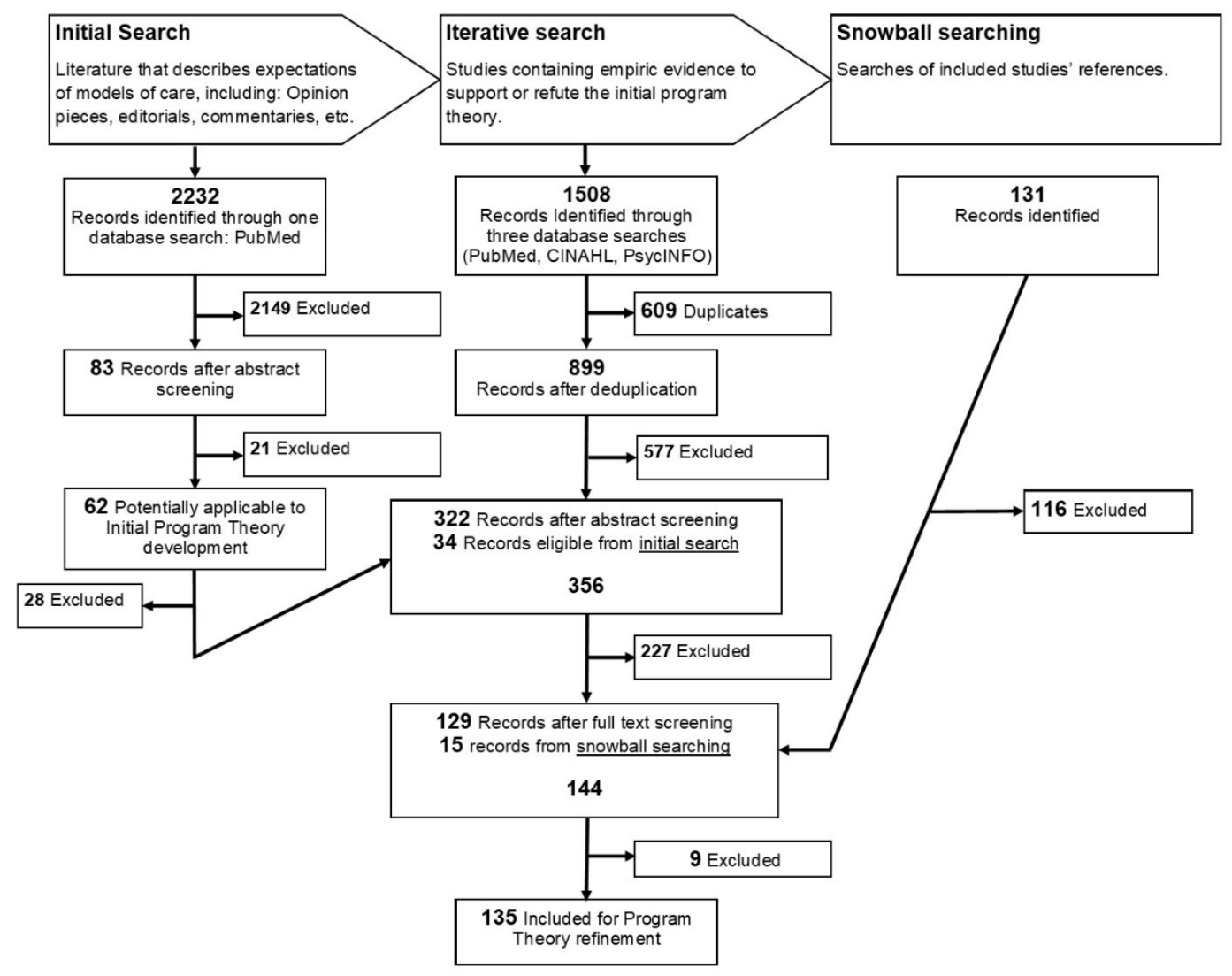




\section{Contextual Questions}

The Contextual Questions (CQs) were addressed by existing reviews and opinion pieces and through stakeholder input. A list of articles used to address the CQs can be found in Appendix D. The effectiveness questions for models and resources are addressed as part of the realist review (KQs 1 and 2) where we describe the evidence suggesting in what contexts and via what mechanisms models of care that include primary care and resources can be effective.

\section{CQ1. How is effectiveness defined and measured for survivorship care models for adult survivors of childhood cancer?}

We identified, through Stakeholder engagement, a number of ways that effectiveness of survivorship care is measured:

- Loss to followup

- Surveillance for long-term and late effects of original cancer treatment

- Concordance with guidelines

- Communication, transfer of information, and care coordination

- Survivor returned to informed primary care providers; survivors able to identify knowledgeable providers

- Informed survivors who know what they need

- Survivors connected with needed resources and services

- Quality of life

- Morbidity

- Costs

- Mortality

CQ2. What are the models of survivorship care for adult survivors of childhood cancer?

\section{a. Which of these models include primary care?}

i. What is the evidence of effectiveness of the different models that include primary care?

There is no consistent taxonomy for survivorship models of care, and models of care are rarely specifically selected in practice. The literature identified in this review generally provided evidence regarding who gets seen where and what care they receive (patterns of care) rather than formal evaluations of specific models of care.

For example, the American Society of Clinical Oncology (ASCO) describes eight different models of care, classified primarily based on provider(s) and setting: oncology specialist care, multi-disciplinary survivorship clinics, disease/treatment-specific survivorship clinics, general survivorship clinics, consultative survivorship clinics, integrated survivorship clinics, community generalist model, and shared-care (Table 1). ${ }^{21}$ All eight of these models may incorporate primary care provider (PCP) involvement. This listing is not specific to childhood cancer survivors, but it applies to this population.

However, the models as described by ASCO are rarely reflected in practice and even more rarely formally evaluated in research. Rather, what is seen in the literature (and experienced by survivors) are more often patterns of care that occur not by design but owing to circumstance. Research studies generally have data on patterns of receipt of care for classification of 
Table 1. Eight models of cancer survivorship care as described by ASCO

\begin{tabular}{|c|c|}
\hline Model & Description \\
\hline $\begin{array}{l}\text { Oncology Specialist } \\
\text { Care }\end{array}$ & $\begin{array}{l}\text { - Followup care occurs in the oncology setting with treating oncologist } \\
\text { - Can be implemented in private practice, community hospitals or cancer centers } \\
\text { - Can be implemented in disease-specific clinics or modality-specific practices } \\
\text { - Communication and coordination with PCP }\end{array}$ \\
\hline $\begin{array}{l}\text { Multi-Disciplinary } \\
\text { Survivorship Clinic }\end{array}$ & $\begin{array}{l}\text { - Care provided by a specialized team (e.g., oncologist, psychologist, cardiologist) in a } \\
\text { separate clinical area } \\
\text { - Can be implemented at cancer centers and other facilities with extensive resources } \\
\text { - Ideal patient populations include: pediatric cancer survivors, AYA survivors and adult } \\
\text { survivors of pediatric cancer* } \\
\text { - Communication and coordination with PCP }\end{array}$ \\
\hline $\begin{array}{l}\text { Disease/Treatment- } \\
\text { Specific Survivor } \\
\text { Clinic }\end{array}$ & $\begin{array}{l}\text { - Care can be provided by a physician, NP, PA, or multispecialty team } \\
\text { - Care provided in oncology setting } \\
\text { - Can be developed for a common diagnosis, such as breast cancer, or treatment } \\
\text { modality, such as stem cell transplant } \\
\text { - Can be developed in private practice, community hospital, or cancer center } \\
\text { - Communication and coordination with PCP }\end{array}$ \\
\hline $\begin{array}{l}\text { General Survivorship } \\
\text { Clinic }\end{array}$ & $\begin{array}{l}\text { - Care can be provided by MD, NP, or PA (not multispecialty) } \\
\text { - Can be implemented at a cancer center, community hospital, or private practice } \\
\text { - Communication and coordination with PCP }\end{array}$ \\
\hline $\begin{array}{l}\text { Consultative } \\
\text { Survivorship Clinic }\end{array}$ & $\begin{array}{l}\text { - Care can be provided by MD, NP, or PA } \\
\text { - One-time visit with no followup, but has potential for another visit } \\
\text { - Ideal for providing treatment summary and care plan } \\
\text { - Can be implemented at a cancer center, community hospital, or private practice } \\
\text { - Communication and coordination with PCP }\end{array}$ \\
\hline $\begin{array}{l}\text { Integrated } \\
\text { Survivorship Clinic }\end{array}$ & $\begin{array}{l}\text { - Embedded in the treatment focused oncology setting } \\
\text { - Care can be provided by an MD, NP, or PA } \\
\text { - Care is ongoing } \\
\text { - Can be implemented at a cancer center, community hospital, or private practice } \\
\text { - Provider communicates and coordinates care with PCP and specialists as needed* }\end{array}$ \\
\hline $\begin{array}{l}\text { Community } \\
\text { Generalist Model }\end{array}$ & $\begin{array}{l}\text { - Survivorship care is provided by } \mathrm{PCP}, \mathrm{NP} \text {, or PA } \\
\text { - Care setting can be at a healthcare system or private practice } \\
\text { - Communication and coordination with PCP }\end{array}$ \\
\hline $\begin{array}{l}\text { Shared-Care of } \\
\text { Survivor }\end{array}$ & $\begin{array}{l}\text { - Care for all survivors is coordinated between oncology specialist, and PCP generalists* } \\
\text { Without Transition } \\
\text { - Followup care occurs in oncology setting } \\
\text { - Can be implemented at a cancer center, community hospital, or private practice } \\
\text { With Transition } \\
\text { - Consultation with the oncology specialists occurs as needed } \\
\text { - At a predetermined time, care is transitioned to the PCP only }\end{array}$ \\
\hline
\end{tabular}

${ }^{*}$ related to childhood cancer survivors

ASCO = American Society of Clinical Oncology; AYA = adolescent/young adult; $\mathrm{MD}=$ medical doctor; NP = nurse practitioner; $\mathrm{PA}=$ physician's assistant; $\mathrm{PCP}=$ primary care provider. 
models (rather than studying formal models), and these studies tend to use broader categories. For example, using data from the Childhood Cancer Survivor Study, Nathan et al. organized receipt of healthcare as follows: no healthcare, general medical care, general survivor-focused care, and risk-based survivor-focused care. ${ }^{7}$ Oeffinger et al. focused on healthcare system interactions: general contact, general physical examination, cancer-related medical visit, and cancer center visit. ${ }^{22}$ Mueller et al. organized care by provider type seen: PCP, specialty care physician, nurse practitioner/physician's assistant, and survivorship clinic team. ${ }^{23}$ Surveys of pediatric oncologists, family physicians, and general internists have asked about categories such as continued care in pediatric oncology, referral to primary care, shared-care with primary care, and followup through a specialized long-term followup program. ${ }^{24-26}$ One systematic review simply categorized models as general practitioner (GP) led versus shared care between a GP and pediatric oncology or a late-effects clinic. ${ }^{27}$ Thus, the taxonomy as proposed by ASCO is more theoretical than practical or evaluable.

Therefore, in this review, we aimed to identify the defining characteristics of models of survivorship care, based on how they occur in practice and how they are presented in the literature. In discussions, Stakeholders described several aspects defining different models of care, including:

- Longitudinal versus one-time clinics

- Location where care is delivered (e.g., cancer center clinics vs. community care)

- Who delivers the care (e.g., doctor, advance practice provider, nurse, or combination thereof)

- Degree of specialized survivorship knowledge - applies to both PCPs and oncologists

- Role of PCPs who specialize in care of cancers survivors and may be integrated in a cancer center, located at a community hospital, or in a private practice

- Either solo or part of an internal medicine-based survivorship clinic

Using the information from the literature and discussion with Stakeholders, we defined four dimensions describing models of survivorship care:

- Particular expertise in survivorship? [yes/no/unclear]

○ Is the survivorship expert trained in oncology, primary care, other, unclear?

Is the survivorship expertise MD, NP/PA, multidisciplinary, unclear?

- Role of PCP [main provider of survivorship care, provides survivorship care under the guidance of survivorship expert, provides primary care with no particular attention to survivorship, unclear]

- Access to academic/cancer center support for survivors and/or providers [yes/no/unclear]

- Consultative versus longitudinal or unclear 
CQs 3 and 4. What survivorship care resources are available for adult survivors of childhood cancer and their families or to providers who care for adult survivors of childhood cancer?

\section{a. What are the intended outcomes of the different resources available for adult survivors of childhood cancer and their families or their care providers? \\ b. What is the evidence of effectiveness of the different resources available for adult survivors of childhood cancer and their families or their care providers?}

\section{c. What are the monetary costs to access these resources?}

In contrast to the taxonomy for the models of survivorship care, the taxonomy for the tools/training/processes/other resources (hereinafter "resources") is more straightforward. Surveys conducted by Nathan et al. and Suh et al. have asked about the value of options such as survivor-specific standardized letters; long-term followup guidelines; expedited routes of contact for consultation, re-referral, and support services; and websites, medical education, and pamphlets. ${ }^{25,26}$ A systematic review by Singer et al. identified a well-organized transition, a treatment summary, a survivorship care plan (SCP), the education of generalist providers, and guidelines as components of successful followup. ${ }^{27}$

We identified 40 resources available to both survivors and providers (see Appendix E for full listing and description of each resource identified). Of these, 23 resources are survivor-specific, 12 are provider/researcher-specific, and 5 are meant for use by both. In addition, we identified nine U.S. guidelines ( 8 physician-specific, and 1 survivor-specific), and 6 international guidelines ( 5 physician/researcher-specific, and 1 meant for both). Based on feedback from the Stakeholders we identified resources that were free to both survivors and providers. According to our Stakeholders, resources are most helpful if they are easy to access, user-friendly, known to survivors and providers, from trustworthy sources, and valued by survivors and their families (e.g., asking for updates to treatment summary).

\section{Development of Initial Program Theory}

We developed an initial program theory based on input from the Stakeholders and a review of the literature. The final products from this initial process are a variable list and initial program theory, which are shown in Appendixes F and G and Figure 3, respectively. Below, we describe the iterative process that produced these final products. In our discussions with Stakeholders, we used the term 'patient' but use the term 'survivor' throughout the report.

\section{Discussion With Stakeholders}

In our first round of discussions, the Stakeholders provided their input on (1) what models of care and resources are available to support survivorship care for childhood cancer survivors and (2) how effectiveness is defined for these models and resources. Based on this initial round of discussions, we also determined that this realist review would focus on adult survivors of childhood cancer only, as the models of care and resources for survivors while still children or adolescents are substantially different.

The Stakeholders identified a number of factors that influence the delivery of survivorship care to childhood cancer survivors. The fractured U.S. healthcare system and availability of 
financial and other resources were identified as key variables at the system, provider, and survivor level. In addition, coordination of care - among primary care and specialty providers, and between providers and survivors - influences the care that is delivered/received and whether and when transition to primary care occurs. The Stakeholders also noted the importance of survivor-specific characteristics, such as developmental age and time since diagnosis, complexity of the diagnosis and treatment(s), current effects of treatment, and risk for late effects.

In terms of effectiveness, the Stakeholders identified mortality, morbidity, quality of life, and costs as the key final outcomes of interest, summarized as "Survivors live longer and feel better through high-value care." They also noted a number of intermediate outcomes, including loss to followup; surveillance for late effects of original cancer treatment and concordance with guidelines; communication, transfer of information, and care coordination; returning survivors to an informed PCP/helping survivors identify knowledgeable providers; ensuring survivors know what they need; and connecting survivors with resources and services. Some additional factors related to resource effectiveness include that they be easy to access, user-friendly, known, and trusted. In combination, the intended effects of the models of care and resources were summarized as "Survivors feel confident about sharing their history, know their risks, recognize symptoms and problems, understand the care they need, are aware of the resources available to help them, and can access relevant care and services."

\section{Literature Review}

We searched the literature for existing mid-range theories that could inform our understanding of the relationships among the key factors identified by the Stakeholders. We also reviewed editorials, commentaries, qualitative, and mixed methods studies to further elucidate our understanding of the key factors, as well as the purported mechanisms and outcomes, for childhood cancer survivorship models of care and resources.

The Andersen Behavioral Model of Health Services $\mathrm{Use}^{28}$ best fit the identified factors from the initial discussions with Stakeholders. In particular, this version of the Behavioral Model of Health Services Use describes how environmental factors (healthcare system, external environment) relate to population characteristics (predisposing characteristics, enabling resources, need) that influence health behavior (personal health practices, use of health services) that lead to outcomes (perceived health status, evaluated health status, consumer satisfaction).

With minor modifications, we were able to fit the key factors identified by the Stakeholders into the Andersen model categories. Modifications included referring to "individual characteristics" rather than "population characteristics," renaming "enabling resources" as "facilitators/barriers," separating survivor and provider health behaviors, and adding "cost" as an outcome.

We then organized our quantitative literature review according to the categories from the modified Andersen model (e.g., environment, individual characteristics, health behaviors, and outcomes). The goal was to identify variables in each of these categories that describe and affect the contexts, mechanisms, and outcomes of childhood cancer survivorship models of care and resources.

\section{Developing Initial Program Theory}

As shown in Appendixes F and G (initial program theory iterations) and in Figure 3 (refined program theory), we have separated the health system/provider factors (shown in yellow) from the patient factors (shown in blue), with the overall environment (shown in white) as the 
background. The models of care and resources sit at the intersection of health system/provider factors and patient factors and are shown in green. We describe the healthcare system attributes and provider factors in yellow and the patient characteristics and needs in blue. The modifiable facilitators/barriers on both the health system/provider side and survivor side are shown in peach. Intermediate outcome and process measures (provider health practices; survivor health service use/behaviors) are displayed in light gray, with the final outcomes displayed in dark gray. Each of these aspects of the program theory are described in more detail below.

On the health system/provider side, healthcare system factors (e.g., degree of fragmentation and integration, availability of financial and other resources, availability of needed specialists) set the stage for provider factors, including characteristics, facilitators/barriers, and practices. Key provider characteristics include specialty (e.g., oncology, primary care) and years in practice. The provider facilitators and barriers of survivorship care include financial and other resources (e.g., staffing, time, reimbursement); providers' relationships with survivors; willingness to transition/accept care for cancer survivors; knowledge about survivorship, generally, and particular survivor's needs; awareness of survivorship resources/guidelines; comfort with treating childhood cancer survivors; and communication/care coordination between and among providers and survivors. Provider health practices include returning survivors to an informed PCP/helping survivors identify knowledgeable providers (and PCPs accepting survivors into their practice and learning about their survivorship needs), connecting survivors with resources and services, conducting guideline-concordant surveillance for long-term and late effects, managing symptoms/late effects, educating survivors about late effects, assessing psychosocial needs and providing psychosocial support, counseling survivors on healthy behaviors, coordinating care, and referring to appropriate specialists (medical, legal, financial).

On the survivor side, key characteristics identified include age at treatment and developmental age, time since and complexity of diagnosis and treatment, race and gender, life transitions (e.g., moving), and genetics. Key needs are the presence of chronic medical conditions and the risk for/severity of late effects. Survivor facilitators/barriers of survivorship care include financial and other resources (e.g., insurance, time, transportation), willingness to transition away from cancer providers, knowledge about their treatment and followup care needs, psychosocial factors (e.g., fear of recurrence, anxiety, depression), autonomy/personal responsibility/self-reliance, and degree of family/parental/partner support for followup care. Relevant survivor health services use includes receiving surveillance for long-term/late effects of original cancer treatment and preventive care; receiving social support, nutritional, rehabilitative, and fertility preservation services; emergency department visits and hospitalizations; and whether the survivor is lost to followup. Health behaviors include use of alcohol/tobacco/drugs and physical activity.

The provider health practices and survivor health services use/behaviors represent the intermediate outcomes as described by the Stakeholders "Survivors feel confident about sharing their history, know their risks, recognize symptoms and problems, understand the care they need, are aware of the resources available to help them, and can access relevant care and services." These outcomes lead to the final outcomes of health status, satisfaction, and costs, or as described by the Stakeholders, "Survivors live longer and feel better through high-value care."

This initial program theory was shared with the Stakeholders in a second round of discussions. The Stakeholders broadly supported the theory and felt that it accurately and comprehensively described the influence of models of care and resources for childhood cancer survivors. They suggested minor additions to the list of variables. For example, on the health- 
system/provider side, they noted the impact of crisis events such as the COVID-19 pandemic, and how these can influence care delivery in important ways. They suggested that concordance of race, language, and culture between providers and survivors could be important and recommended that the general category of survivor social determinants of health be added. Regarding financial aspects, they noted that incentives (both financial and related to quality programs) could play a role on the provider side and that financial costs on the survivor side include reimbursement and ability to pay their co-pays. For survivors, coordination of multiple specialists was identified as a particular challenge. Other comments primarily related to the refinement of how variables were listed.

\section{Refinement of Initial Program Theory}

To refine the initial program theory, we sought quantitative evidence and evaluated whether the evidence supported, refuted, or suggested revisions to our initial program theory. Here, we describe how we updated the variable list (Appendix F) and revised the initial program theory (Appendix G) based on the quantitative literature to develop our refined program theory (Figure 3).

There was at least some evidence addressing almost all of the variables in our initial list. The variables most commonly found in the literature we reviewed included oncology versus primary care specialty; cancer type and complexity of diagnosis; survivor age, race, and gender; survivor and provider financial and other resources; survivor and provider knowledge about childhood cancer survivors' needs generally, and about particular survivor's needs; provider comfort with treating childhood cancer survivors; communication and coordination between and among providers and survivors; delivery/receipt of prevention and surveillance of long-term and late effects of original cancer treatment; and quality of life/health status and satisfaction. Variables that were seen less frequently include crisis events, genetics, emergency department visits, hospitalizations, and costs.

The literature also identified some factors that we added to our list of variables. Under Environment, we added regional differences. Under Provider Characteristics, we added gender and survivor volume. Under Survivor Characteristics, we added cancer type, current age, marital status, and work status; we also clarified that time since diagnosis represents both longitudinal (5 years vs. 10 years from diagnosis) and calendar (e.g., diagnosed in 1985 vs. 1999) time. Under Survivor Facilitators/Barriers, we added awareness and availability of (culturally appropriate) resources.

The most important substantive change made to the initial program theory was the addition of an intermediate outcome focused on PCPs. Specifically, our initial steps had identified as the intermediate outcome that "Survivors feel confident about sharing their history, know their risks, recognize symptoms and problems, understand the care they need, are aware of the resources available to help them, and can access relevant care and services." The evidence in the literature demonstrated that there is also an important provider component as an intermediate outcome, which is 'PCPs understand a survivor's history, know the survivor's risks, recognize symptoms and problems, understand the care survivors need, are aware of the resources available to help them, and can access relevant care and services."

In the final round of Stakeholder discussions, we reviewed these findings with the Stakeholders, who reported that our results were consistent with their understanding based on their experiences with and expertise in childhood cancer survivorship care. 
Figure 3. Refined program theory

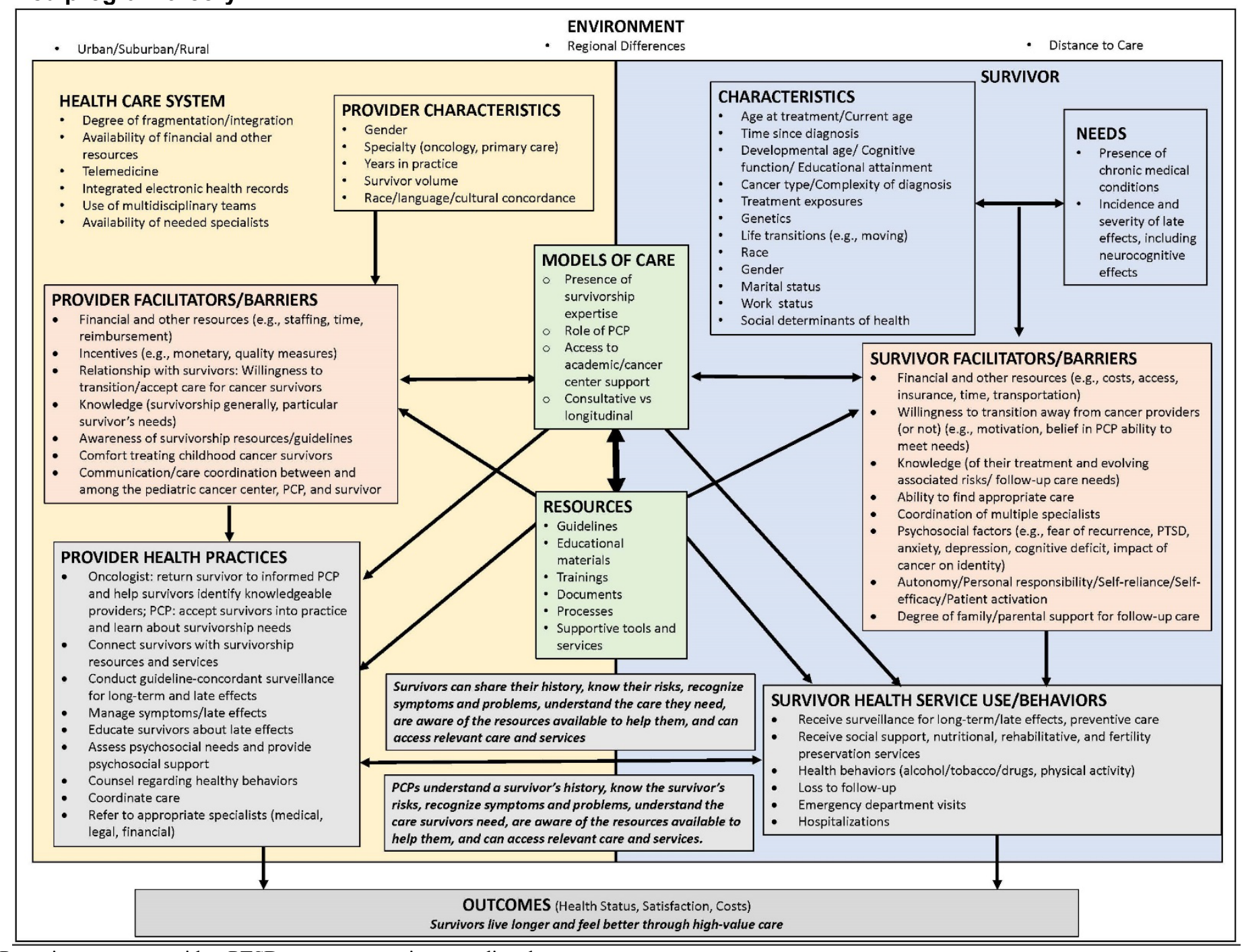

$\mathrm{PCP}=$ primary care provider; $\mathrm{PTSD}=$ post-traumatic stress disorder. 


\section{Realist Review Key Questions 1 and 2}

Key Question (KQ) 1. For whom and under what circumstances could different survivorship care models for adult survivors of childhood cancer (cancer diagnosed prior to age 21 years old) that include primary care be effective?

a. What are the key mechanisms by which these models could be effective?

b. What are important contexts that determine whether different mechanisms could be effective?

KQ2. For whom and under what circumstances could different survivorship care resources for adult survivors of childhood cancer be effective in achieving their intended outcomes?

a. For survivors and their families

i. What are the key mechanisms by which these resources could lead to their intended outcome?

ii. What are important contexts that determine whether different mechanisms could lead to outcomes?

b. For care providers

i. What are the key mechanisms by which these resources could lead to their intended outcome?

ii. What are important contexts that determine whether different mechanisms could lead to outcomes?

\section{Development of Context-Mechanism-Outcome Hypotheses}

To address the realist review KQs related to the contexts and mechanisms associated with effective models of care that include primary care (KQ1) and of resources (KQ2) used to achieve outcomes, we abstracted information supporting or refuting our program theory from the quantitative literature and developed seven context-mechanism-outcome (CMO) hypotheses. Because the CMOs related to the models of care and resources were closely linked, we addressed these KQs jointly. We also noted that the realist review KQ regarding models of care was not whether models of care that include primary care are effective for adult survivors of childhood cancer but, rather, in what contexts and via what mechanisms they could be effective.

These CMO hypotheses were primarily developed based on evidence from literature describing childhood cancer survivors (both during childhood/adolescence/young adulthood and 
adulthood), though we also drew from evidence regarding adult survivors of adult cancers where relevant and informative. For each $\mathrm{CMO}$, we provide example evidence from the literature that supports, and in some cases, refutes, the hypothesis. As with all realist reviews, the evidence is provided for illustrative purposes and is not intended to be a comprehensive summary.

\section{Context-Mechanism-Outcome Hypotheses Focused on Survivor Intermediate Outcomes}

Four CMO hypotheses focus on the survivor intermediate outcome of "Survivors feel confident about sharing their history, know their risks, recognize symptoms and problems, understand the care they need, are aware of the resources available to help them, and can access relevant care and services," as shown in Table 2.

Table 2. Context-mechanism-outcome hypotheses regarding how models of care that include primary care could be effective, focusing on intermediate outcomes for the survivor

\begin{tabular}{|c|c|c|c|}
\hline In the CONTEXT of & This MECHANISM ... Produces & $\begin{array}{l}\text { OUTCOME } \\
\text { (Intermediate) }\end{array}$ & $\begin{array}{l}\text { OUTCOME } \\
\text { (Final) }\end{array}$ \\
\hline $\begin{array}{l}\text { A) the availability of } \\
\text { survivorship care plans, } \\
\text { guidelines, and other } \\
\text { resources }\end{array}$ & $\begin{array}{l}\text { A1) improved survivor knowledge } \\
\text { A2) information available to } \\
\text { share with PCP to inform delivery } \\
\text { of survivorship-related care }\end{array}$ & $\begin{array}{l}\text { Survivors can share } \\
\text { their history, know their } \\
\text { risks, recognize } \\
\text { symptoms and } \\
\text { problems, understand } \\
\text { the care they need, are } \\
\text { aware of the resources } \\
\text { available to help them, } \\
\text { and can access relevant } \\
\text { care and services. }\end{array}$ & $\begin{array}{l}\text { Survivors live } \\
\text { longer and feel } \\
\text { better through } \\
\text { high-value care. }\end{array}$ \\
\hline $\begin{array}{l}\text { B) healthier survivors } \\
\text { (perceived or actual) }\end{array}$ & $\begin{array}{l}\text { B) less perceived/actual need for } \\
\text { survivorship-related care }\end{array}$ & $\begin{array}{l}\text { Survivors can share } \\
\text { their history, know their } \\
\text { risks, recognize } \\
\text { symptoms and } \\
\text { problems, understand } \\
\text { the care they need, are } \\
\text { aware of the resources } \\
\text { available to help them, } \\
\text { and can access relevant } \\
\text { care and services. }\end{array}$ & $\begin{array}{l}\text { Survivors live } \\
\text { longer and feel } \\
\text { better through } \\
\text { high-value care. }\end{array}$ \\
\hline $\begin{array}{l}\text { C) survivors engaged in } \\
\text { healthcare system }\end{array}$ & C) improved knowledge & $\begin{array}{l}\text { Survivors can share } \\
\text { their history, know their } \\
\text { risks, recognize } \\
\text { symptoms and } \\
\text { problems, understand } \\
\text { the care they need, are } \\
\text { aware of the resources } \\
\text { available to help them, } \\
\text { and can access relevant } \\
\text { care and services. }\end{array}$ & $\begin{array}{l}\text { Survivors live } \\
\text { longer and feel } \\
\text { better through } \\
\text { high-value care. }\end{array}$ \\
\hline $\begin{array}{l}\text { D) survivor confidence in } \\
\text { PCPs }\end{array}$ & D) willingness to transition care & $\begin{array}{l}\text { Survivors can share } \\
\text { their history, know their } \\
\text { risks, recognize } \\
\text { symptoms and } \\
\text { problems, understand } \\
\text { the care they need, are } \\
\text { aware of the resources } \\
\text { available to help them, } \\
\text { and can access relevant } \\
\text { care and services. }\end{array}$ & $\begin{array}{l}\text { Survivors live } \\
\text { longer and feel } \\
\text { better through } \\
\text { high-value care. }\end{array}$ \\
\hline
\end{tabular}

$\mathrm{PCP}=$ primary care provider. 


\section{Context A: SCPs, Guidelines, and Other Resources}

In the literature, we found that the availability of SCPs, guidelines, and other resources led to the survivor-focused intermediate outcome via two mechanisms: (1) survivors have improved knowledge regarding their care needs and (2) survivors have information to share with their PCPs to inform delivery of survivorship-related care.

We also identified four sub-themes related to the sharing of information through these resources: (1) improved knowledge can be both beneficial and detrimental, (2) resources have bigger impacts on survivors with lower knowledge at baseline, (3) issues regarding resource "dose," and (4) gender differences in engagement with and impact of resources.

\section{Mechanism A1: Improved Knowledge for Survivors}

Regarding the first mechanism, in a survey of 1,395 adolescent/young adult survivors, Shay et al. found that SCPs and/or followup care instructions were significantly associated with lower odds of survivors reporting unmet needs around information about topics such as late effects of treatment, fertility, and recurrence concerns. ${ }^{29}$ As another example, childhood cancer survivors 2-years post-treatment who were given a passport card describing diagnosis, treatment, risks, and recommended followup were more likely to demonstrate improved knowledge versus survivors without a passport. ${ }^{30}$ A third study, conducted by Yan et al., found that possession of an SCP among high-risk survivors was associated with increased adherence to breast, skin, and cardiac surveillance. ${ }^{31}$ These studies support the role of SCPs, guidelines, and other resources in improved knowledge for survivors.

\section{Mechanism A2: Transfer of Information to PCPs}

Regarding the second mechanism, there was also evidence that survivors valued the information from SCPs and other resources to share with their other providers. For example, in a study of 111 adult survivors of pediatric and young adult cancer, 95 percent reported understanding everything on the treatment summary, 83 percent found it extremely or moderately valuable, and 95 percent found the SCP helpful in understanding the plan for their care. ${ }^{32}$ In addition, of 30 respondents who had visited an outside provider since getting the SCP, one-third gave the provider a copy of the form and 44 percent gave a copy to someone in their personal circle. However, intentions to share the SCP do not always translate into action. In a cohort study of 20 families of a child with acute lymphoblastic leukemia, 95.75 percent of parents reported intending to share the SCP with someone, but only 60.9 percent had done so at the third followup, and only 35 percent of those with a PCP had shared it with them by Time $3 .{ }^{33}$

In a prospective one-arm study assessing SCP and information provision in 62 childhood cancer survivors at increased risk for late effects, nearly half of those who had seen a physician since SCP receipt had shared it; most who had not seen a physician planned to share the SCP when they did. ${ }^{34}$ Further, the investigators found that PCPs seemed too busy to be involved in the study or to utilize the resources offered to them directly, but they ordered tests when the survivor presented them with information on risks. In a study of 5,661 adult survivors of childhood cancer, Steele et al. found that discussing cancer-related risks with a doctor is the strongest predictor of getting screened for late effects and that the physician's access to the survivor's cancer treatment summary significantly predicted screening for relevant health risks. ${ }^{35}$ Based on these findings, the authors discuss the importance of communication between survivors and providers regarding receipt of appropriate care and the role of resources, such as treatment summaries, in promoting this communication. 
Key points that emerge from this literature are that (1) survivors see value in the information, and intend to share it, but do not always do so and (2) providers have limited capacity to engage with resources but, when information is shared with them in an effective way, it can lead to improved care delivery.

Context A, Subtheme 1: Benefits and harms of information. An important theme in the literature about resources and the information they provide is that they can be both beneficial and detrimental. For example, the Spain et al. study of adult survivors of pediatric and young adult cancer found that 14 percent reported being concerned by the SCP as a whole, and 28 percent were concerned by potential late effects. ${ }^{32}$

A randomized controlled trial (RCT) of SCPs in gynecologic cancer survivors (not childhood cancer survivors) investigated this issue in greater depth. In this Dutch study of an electronic medical record-generated SCP for survivors of ovarian and endometrial cancer, endometrial cancer survivors in the SCP arm reported greater concern about their illness, more emotional effects, and more symptoms. ${ }^{36}$ Similarly, the ovarian cancer survivors in the SCP arm reported less trust that the treatment would cure their disease. ${ }^{37}$ The authors noted that these negative outcomes are not necessarily bad. For example, the endometrial cancer survivors had more cancer-related contact with their PCPs, which the authors speculated relates to the survivors' greater awareness of cancer-related symptoms and the possibility that having the SCPs empowered them to seek out the necessary support. ${ }^{36}$ They also suggest that healthcare providers may be reluctant to share information about potential late effects to avoid such negative consequences, but that avoidance of the issue may limit the survivor's awareness and empowerment. For the ovarian cancer survivors, the authors note that the decreased belief in the potential for cure gleaned from the SCP may be more realistic, but that it is an issue providers may be reluctant to address. ${ }^{37}$

In an analysis of 7,917 cancer survivors (unspecified if childhood or adult cancer survivors) who participated in the Cancer Survivorship Module of the 2010 Behavioral Risk Factor Surveillance System, Jabson et al. found a similar association. Specifically, they found that survivors who had received a treatment summary, followup care instructions, or both were more likely to report pain. ${ }^{38}$ The authors note that the documents might heighten cancer survivors' awareness and reporting of cancer-related pain; alternatively, it could be that patients who undergo more extensive treatment are more likely to both experience pain and receive a treatment summary and followup care instructions. A different analysis by Jabson, this one in 3,541 cancer survivors who participated in a 2010 LIVESTRONG online survey, had different findings. ${ }^{39}$ In this population, they found that survivors who received followup care instructions reported 25 percent fewer late effects. In this paper, they hypothesized that cancer survivors who receive followup care instructions become more aware of the symptoms of late effects sooner, leading them to obtain care earlier and resulting in them reporting fewer problems.

The authors of the Dutch RCT in gynecologic cancer survivors conducted a followup analysis that examined how different preferences for information affected the impact of SCPs. ${ }^{40}$ They compared "monitors," who desire information about their disease to "blunters," who avoid information. SCPs were more beneficial to monitors across the board, but particularly those who did not have easy access to other information sources, such as the internet. For blunters, those in the SCP arm reported a greater impact of the disease on life and more concerns about the illness compared with blunters in the control arm.

These studies raise two Key Questions: (1) when harms are found, is it because the information is causing unnecessary concern, or leading to a more realistic understanding of the 
implications of the cancer and (2) to what extent should delivery of information be tailored to the preference of the survivor?

Context A, Subtheme 2: Resources have greater impact for survivors with lower baseline knowledge. Several studies have found that resources improved knowledge more in survivors who knew less. For example, in one study of adult survivors of childhood cancer, new patients were more likely to report learning new information from the SCP compared with return patients. ${ }^{32}$ In a different study, which tested a survivorship clinic visit intervention in 369 adult survivors of childhood cancer, survivors with the lowest knowledge of therapy and therapyrelated health risk at baseline had the greatest gains. ${ }^{41}$

Papers with similar findings in adult survivors of adult cancers have posited that the failure of resources to demonstrate impacts may be due in part to high levels of knowledge and/or few or no needs in the populations being studied. ${ }^{42,43}$

These findings raise the question of whether resources should be targeted to survivors who have information deficits or needs and as described above, for whom the resource will be beneficial rather than detrimental.

Context A, Subtheme 3: Resource "dose." An important consideration in implementing resources is that they deliver a sufficient "dose" to be effective. Several studies noted that failure to find an effect of the studied resource may have been related to an insufficient dose. For example, Hudson et al. found that a brief, broad-based risk counseling intervention did not achieve a substantial long-term change in knowledge, health perceptions, or health practices in childhood cancer survivors. ${ }^{44}$ Similarly, Steele et al. found that their "relatively weak intervention dose" of a targeted (not tailored) page of information in a newsletter did not lead to increased medical followup in at-risk pediatric cancer survivors. They also noted that tailored information is more consistently effective, though effects are small. ${ }^{35}$

As an example from studies of adult survivors of adult cancers, Turner et al. suggested that timing or insufficient "dose" may have led to the null findings in their trial of a Head and Neck Cancer Survivor Self-Management Care Plan. ${ }^{45}$

More evidence is needed to confirm whether more intensive interventions lead to greater resource effectiveness. However, even if confirmed, as discussed below regarding providers, practical considerations limit how much time and effort a resource can involve.

Context A, Subtheme 4: Gender differences. One final subtheme identified in the literature was differences in gender regarding engagement with and impact of resources. For example, Hudson et al. found that female childhood cancer survivors had a statistically significantly greater improvement in knowledge following a multi-component risk-counseling intervention versus males, though the difference was small. ${ }^{44}$ Similarly, in the Steele et al. study in childhood cancer survivors comparing two approaches for sharing information via a newsletter, more women ( 72 percent) than men (59 percent) reported reading the newsletter. ${ }^{35}$ In the Oeffinger et al. single-arm study of high-risk childhood cancer survivors, more women than men visited the study website that provided survivorship resources. ${ }^{34}$

These results raise questions regarding whether resources should be tailored to different groups (e.g., males vs. females) based on how they engage and use them.

\section{Context B: Survivors Who Perceive Themselves To Be, or Are Actually, Healthier}

A different context in which models of care that include primary care could be effective for childhood cancer survivors is when survivors perceive themselves to be - or are actually - 
healthier. This CMO hypothesis is consistent with the National Academy of Medicine (NAM) report's recommendation for risk-adjusted followup care. ${ }^{8}$

In one analysis of 6,176 survivors from the Childhood Cancer Survivor Study, survivors who reported no morbidity at baseline were less likely to report receiving care at followup, whereas survivors who reported any chronic health condition at baseline were more likely to report care at followup. ${ }^{46}$ Another analysis of the Childhood Cancer Survivor Study found that survivors who received more intensive therapy; those with a severe, life-threatening, or disabling chronic condition; and those who reported worse cancer-related pain were more likely to have received care. ${ }^{7}$ Klosky et al. found that one factor associated with non-attendance at the St. Jude followup clinic for childhood cancer survivors was having had no additional cancer event. ${ }^{47}$

In a survey of 160 Swiss adolescent/young adult survivors, non-attenders of followup were more likely to rate models of care involving a GP or via telephone/questionnaire higher than attenders. ${ }^{48}$ Because non-attenders were also less likely to report late effects than attenders of followup, we believe this finding provides support to the hypothesis that models of care that include primary care could be effective for survivors who are healthier. In another study of childhood cancer survivors, low perceived need for care was a key factor highlighted for not engaging in care. ${ }^{49}$ Guilcher et al. conducted a survey of 16 pediatric hematology/oncology programs regarding long-term followup care programs for childhood cancer survivors in Canada. They found that the key factors associated with followup in a formal adult late effects program were existing late effects, higher risk for adult onset late effects, and time from completion of therapy; ${ }^{.0}$ one would expect the converse factors to be associated with followup in primary care (or, perhaps, no followup).

This evidence raises two issues regarding the risk-based followup care recommended by the NAM. First, attendance at clinic is not equivalent to need for care. Some low-risk survivors may be unwilling to transfer care if, for example, they do not have confidence in their PCP to provide care (as described below). Second, non-attendance at clinic is not equivalent to no need for care. Some of the survivors classified as non-attenders may not have been fully aware of their risk for late effects. This theory is supported by an RCT that investigated a tailored counseling intervention in 472 Childhood Cancer Survivor Study participants at-risk for cardiomyopathy. ${ }^{51}$ While more survivors (52.2 percent) in the intervention arm completed cardiomyopathy screening compared with controls (22.3 percent), one of the reasons cited for not getting screened was not perceiving it as important. Notably, screening detected cardiomyopathy or other abnormalities consistent with evolving cardiac dysfunction in over 20 percent of those screened.

The key points that emerge from these findings is that survivors who are healthier may be more comfortable with followup in primary care. However, perceived health need is not the same as actual health need, and survivors who do not understand their risks for long-term and lateeffects may not be receiving recommended screening and needed care.

\section{Context C: Survivors Who Are Engaged in the Healthcare System}

Based on the literature, being engaged in the healthcare system (e.g., physician visits) provides another context in which survivors can gain the knowledge they need and receive appropriate care, though certainly not universally. For example, among 106 young adult survivors of childhood cancer, 63.2 percent reported that, during their last clinic visit, a provider discussed any symptoms related to their cancer, with less than half reporting that they promoted adherence to care recommendations, interest in reproduction, social support, and mental health 
issues. ${ }^{52}$ A Swedish study of 213 young adult survivors of childhood acute lymphoblastic leukemia found that respondents who had no regular contact with healthcare services were more likely to report that they had not received knowledge, treatment strategies, or guidance for coping with physical changes. ${ }^{53}$ In addition, satisfaction with level of contact was associated with reporting receipt of knowledge and treatment strategies regarding physical changes - those who were more satisfied were more likely to report receipt. Nevertheless, even among the respondents who were satisfied with the level of contact, only a minority reported receiving sufficient knowledge and treatment strategies about physical changes. Regarding receipt of care, Yan et al.'s study of childhood cancer survivors found that having a cancer-related check-up in the past 2 years was associated with increased surveillance for breast, skin, and cardiac late effects; visiting a doctor more than five times in the past 2 years was associated with increased surveillance for skin and cardiac late effects. ${ }^{31}$ Importantly, the sociodemographic factors consistently associated with childhood cancer survivors not being engaged in care include male sex, lack of insurance, lower income, race (non-White or other), and less education. ${ }^{7,46,47,52}$

These findings provide insights regarding the pathways to appropriate receipt of care; it seems that survivors who are engaged with the healthcare system learn more about their care needs and are more likely to receive appropriate care.

\section{Context D: Survivors Who Have Greater Confidence in Their PCPs}

A final context describes how survivors who are confident in their PCPs might be more willing to transition their care. In the Stakeholder discussions, one of the Stakeholders noted that it is important that the survivor's "emotional/physical escorts" (e.g., their parents or partners) also be confident in primary care for the transition to occur.

The evidence regarding this CMO hypothesis actually supports the converse: survivors are not confident in PCPs and prefer models of care that include cancer specialists. For example, in an Australian survey of 633 parents of childhood cancer survivors, adolescent/young adult survivors, and older survivors of childhood cancer, it was found that hospital-based survivorship care-involving an oncologist or other clinic doctor, cancer survivorship nurse, or team-was the first choice of 97 percent of parents, 88 percent of adolescent/young adults, and 86 percent of older survivors. ${ }^{54}$ They had lower confidence in PCPs. Similar findings were seen in a survey of 160 Swiss adolescent/young adult survivors, who rated medical oncologist involvement most highly for survivorship care. ${ }^{48}$ The authors noted that, even though the respondents were surveyed at least 5 years after diagnosis, their biggest concerns were cancer relapse and occurrence of late effects. They speculate that survivors may perceive medical oncologists as best suited to deal with these issues. An alternate perspective is provided from a survey in the Netherlands of 133 adult survivors of childhood cancer. ${ }^{55}$ In this study, 88 percent of survivors were satisfied with the care given by local family doctors, and only 14 percent thought their local family doctor's knowledge of their medical history was inadequate.

The limited evidence regarding this $\mathrm{CMO}$ hypothesis suggests that work is required to increase the confidence of cancer survivors in PCPs to facilitate models of care that include primary care.

\section{Context-Mechanism-Outcome Hypotheses Focused on Provider Intermediate Outcomes}

Three of our CMO hypotheses focus on the provider intermediate outcome of "PCPs understand a survivor's history, know the survivor's risks, recognize symptoms and problems, understand 
the care survivors need, are aware of the resources available to help them, and can access relevant care and services," as shown in Table 3. Several Stakeholders commented that all three CMO hypotheses relate to PCPs obtaining the knowledge needed to appropriately care for childhood care survivors - either through SCPs, guidelines, and resources; shared-care with an oncologist; or greater experience caring for childhood cancer survivors.

Table 3. Context-mechanism-outcome hypotheses regarding how models of care that include primary care could be effective, focusing on intermediate outcomes for the primary care provider

\begin{tabular}{|c|c|c|c|}
\hline In the CONTEXT of & $\begin{array}{l}\text { This MECHANISM ... } \\
\text { Produces }\end{array}$ & $\begin{array}{l}\text { OUTCOME } \\
\text { (Intermediate) }\end{array}$ & $\begin{array}{l}\text { OUTCOME } \\
\text { (Final) }\end{array}$ \\
\hline $\begin{array}{l}\text { A) the availability of } \\
\text { survivorship care plans, } \\
\text { guidelines, and other } \\
\text { resources }\end{array}$ & $\begin{array}{l}\text { A) information available to } \\
\text { guide the PCP in delivering } \\
\text { survivorship-related care }\end{array}$ & $\begin{array}{l}\text { PCPs understand a } \\
\text { survivor's history, know } \\
\text { the survivor's risks, } \\
\text { recognize symptoms } \\
\text { and problems, } \\
\text { understand the care } \\
\text { survivors need, are } \\
\text { aware of the resources } \\
\text { available to help them, } \\
\text { and can access relevant } \\
\text { care and services. }\end{array}$ & $\begin{array}{l}\text { Survivors live } \\
\text { longer and feel } \\
\text { better through high- } \\
\text { value care. }\end{array}$ \\
\hline $\begin{array}{l}\text { B) PCP shared-care with } \\
\text { oncologist }\end{array}$ & $\begin{array}{l}\text { B) support from the } \\
\text { oncologist to aid the PCP in } \\
\text { delivering survivorship-related } \\
\text { care }\end{array}$ & $\begin{array}{l}\text { PCPs understand a } \\
\text { survivor's history, know } \\
\text { the survivor's risks, } \\
\text { recognize symptoms } \\
\text { and problems, } \\
\text { understand the care } \\
\text { survivors need, are } \\
\text { aware of the resources } \\
\text { available to help them, } \\
\text { and can access relevant } \\
\text { care and services. }\end{array}$ & $\begin{array}{l}\text { Survivors live } \\
\text { longer and feel } \\
\text { better through high- } \\
\text { value care. }\end{array}$ \\
\hline $\begin{array}{l}\text { C) more experience } \\
\text { caring for childhood } \\
\text { cancer survivors }\end{array}$ & $\begin{array}{l}\text { C) greater comfort caring for } \\
\text { childhood care survivors }\end{array}$ & $\begin{array}{l}\text { PCPs understand a } \\
\text { survivor's history, know } \\
\text { the survivor's risks, } \\
\text { recognize symptoms } \\
\text { and problems, } \\
\text { understand the care } \\
\text { survivors need, are } \\
\text { aware of the resources } \\
\text { available to help them, } \\
\text { and can access relevant } \\
\text { care and services. }\end{array}$ & $\begin{array}{l}\text { Survivors live } \\
\text { longer and feel } \\
\text { better through high- } \\
\text { value care. }\end{array}$ \\
\hline
\end{tabular}

$\mathrm{PCP}=$ primary care provider

\section{Context A: SCPs, Guidelines, and Other Resources}

For providers, we found that the availability of SCPs, guidelines, and other resources work through the mechanism of having information available to guide the PCP in delivering survivorship-related care.

Multiple studies have documented the value that PCPs place on resources to support their delivery of survivorship care. In one survey, internists $(n=1,110)$ and family practitioners $(n=1,024)$ rated highly long-term followup guidelines and survivor-specific standardized letters from specialists with followup recommendations for supporting their delivery of survivorship care to childhood cancer survivors. ${ }^{25,26}$ Similarly, another survey of 351 general internal medicine and family practice providers found that more than 90 percent thought it would be useful to receive a treatment summary or SCP, and 86 percent agreed they would follow 
guidelines when caring for adult survivors of childhood cancer. ${ }^{56}$ In a cross-sectional survey of 27 medical oncologists, 13 pediatric oncologists, 122 GPs, and 21 pediatricians in Switzerland, all groups reported a need for standardized protocols ( 85 to 91 percent) and specialized training ( 55 to 73 percent) for caring for childhood cancer survivors. ${ }^{57}$ These resources are also endorsed as a key enabling factor for shared care. Among 233 GPs in the Netherlands who had taken a postgraduate course on late effects of childhood cancer treatment, two of the main requirements for participation in shared followup care were availability of guidelines (64 percent) and sufficient information about the survivor's medical history (37 percent) ${ }^{58}$

There is also some evidence that PCPs use these resources and that the resources promote quality survivorship care. Among PCPs caring for childhood cancer survivors who recalled receiving an SCP as part of a research study, 75 percent reported often or always reviewing the plan, and 42 percent reported discussing the SCP with the survivor; further, 48 percent found the treatment summary very helpful, and 56 percent found the followup surveillance recommendations helpful. ${ }^{59}$ Yan et al. found that PCP possession of an SCP was associated with increased adherence to breast and colorectal screening among childhood cancer survivors at average risk, though less than half of high-risk survivors received recommended surveillance. ${ }^{31}$

Despite the potential value of these resources, there is also evidence that they currently have limited reach and effectiveness. In a survey of 351 US general internal medicine and family practice providers regarding care for adult survivors of childhood cancer, respondents endorsed the value of SCPs and guidelines, but approximately 85 percent reported never receiving a cancer treatment summary or SCP, and 93 percent reported never using the Children's Oncology Group (COG) late effects guidelines. ${ }^{56}$ Further, only 40 percent of providers were aware of the guidelines, and less than 40 percent felt their training was adequate to recognize late effects. However, Yan et al.'s Childhood Cancer Survivor Study analysis reported substantial improvements in adherence to the COG guidelines between 2003 and 2016 and speculated that physician awareness of COG guidelines may be growing. ${ }^{31}$ In a study of SCPs for childhood cancer survivors published in 2017, a survey of 134 PCPs found that only 31 percent of the PCPs felt very comfortable providing the surveillance recommended on the SCP, 19 percent felt very comfortable using the SCP to provide recommended surveillance for neurocognitive late effects, and 3 percent felt very comfortable providing all aspects of survivorship care. ${ }^{59}$ In discussing these results, the authors noted the limitations of passive SCP distribution and the need to investigate ways to deliver information to PCPs to better address their knowledge needs.

This evidence suggests that there is potential value for SCPs, guidelines, and other resources in promoting effective childhood cancer survivorship care, but that their potential has yet to be fully realized. As relates to this point, we identified three sub-themes: 1) lack of awareness of the resources; 2) role of the electronic health record (EHR) in managing information; and 3) tension between resources delivering a sufficient "dose" and practical considerations regarding what PCPs can take the time to do.

Context A, Subtheme 1: Lack of awareness of resources. An interesting finding from the SCP study of Iyer et al. in childhood cancer survivors was that, even though all PCPs were sent an SCP as part of the study, only 51 percent recalled receiving it. ${ }^{59}$

Donahue et al. obtained the perspectives of 81 PCPs seeing adult breast cancer survivors enrolled in a survivorship clinical trial. ${ }^{60}$ PCPs reported that the main barriers to SCP use were not knowing a plan existed ( 83 percent), not knowing how to find the plan (75 percent), not being able to locate the plan in the patient chart (71 percent), and not knowing to look for the plan (72 percent). Notably, these barriers were reported even though the SCPs are standardly 
housed in the EHR problem list within their institution. Potential facilitators identified by the PCPs included awareness of plan existence ( 85 percent), a standardized location within medical records ( 89 percent), and consistent provision of care plans for all patients ( 81 percent). The PCPs noted that an SCP designed specifically for them, rather than one designed to serve both survivors and PCPs, may be more helpful. In discussing the findings, the investigators speculated that their institution may have yet to reach a "critical mass" of SCP provision that would facilitate PCPs' awareness of and ability to use them. They suggest the need for "primary carecentered design of SCP format and content, location in the EHR, and the ability to 'push' relevant or needed survivorship information to primary care at the right time." Another study that included 21 physicians or dentists of adult survivors of adult cancer found that only 34 percent recalled receiving the SCP or could locate it. ${ }^{61}$ The authors of this study compared the SCP to "a needle in a haystack" for healthcare professionals across institutions who use different EHRs or paper charts.

Knowing that an SCP exists is a critical first step in being able to use it in practice, and EHRs can both help and hinder that process. Below, we discuss the role of EHRs in creating SCPs.

Context A, Subtheme 2: Role of EHRs in adult survivors of adult cancers. A number of studies commented on or highlighted the role of the EHR in creating SCPs. The SCPs used in the Netherlands RCT in gynecologic cancer were auto-populated from the EHR, ${ }^{36,37,62}$ as were the SCPs used in a study of hematopoietic stem cell transplantation survivors. ${ }^{63}$ Morken et al. describe the advantages of using EHRs to create and provide SCPs, including reduced time and resources to compile the information, producing a document that is electronically searchable, and facilitating updates to the plan when needed ${ }^{63}$ However, they also noted that, for EHRs to support the effective use of SCPs, discrete data capture is required, organization policies and technologies must be designed to support clinician needs, and survivorship-related tasks need to be clearly assigned. Perhaps because of these barriers, in surveys of cancer programs and cancer care providers, Birken et al. found that EHR systems used to create SCPs were lacking and/or underused. ${ }^{64,65}$

The potential role of EHRs to promote the creation, delivery, and data management of SCPs requires further exploration.

Context A, Subtheme 3: Resource "dose." Similar to the subtheme described above regarding survivors, there is also a tension between resources delivering a sufficient "dose" to providers and practical considerations regarding what PCPs can take the time to do. For example, as part of a survivorship study, Oeffinger et al. developed a virtual information center for childhood cancer survivors and their providers, but none of the PCPs who agreed to participate in the study visited the website. ${ }^{34}$ In a different study, Costello et al. aimed to implement a telemedicine transition visit with a PCP and childhood cancer survivors joined by a pediatric survivorship clinic team member ${ }^{66}$ However, only 24 of 46 eligible dyads agreed to participate, due in part to PCPs' hesitation with using the study-provided telemedicine equipment. From the adult survivorship literature, there is some evidence that providers prefer more targeted information, such as SCPs that are shorter and directed to PCPs' needs, rather than information directed to both PCPs and survivors. ${ }^{60}$ One Stakeholder raised the issue of whether providers might be more willing to undertake these activities if reimbursement were sufficient.

Similar to what was found with survivors, there is a delicate balance between providing a sufficient "dose" of information to be effective and being realistic regarding the time and effort providers can invest. 


\section{Context B: Shared-Care With Oncologist}

Similar to the reports from survivors above regarding preferences for models of care that include cancer specialists, providers highly endorse shared-care models. Among 1,110 internists and 1,024 family practitioners, 84 percent and 85 percent, respectively, prefer working in collaboration with a cancer center-based physician or long-term followup clinic for childhood cancer survivorship care. ${ }^{25,26}$ Among the 134 PCPs interviewed in another study about childhood cancer survivorship care, 46 percent selected specialty survivorship clinic as the most preferred model of care, followed by 26 percent preferring shared care ${ }^{59}$ In a questionnaire completed by 233 Dutch GPs who had taken a postgraduate course on late effects of childhood cancer treatment, 97 percent were willing to participate in a shared-care model for followup of adult survivors. ${ }^{58}$ Among these respondents, in addition to endorsing the importance of guidelines and relevant information about the survivor's medical history (described above), they also noted the importance of easy access to specialists to support shared care. In a separate study, these authors conducted a survey of 133 adult survivors of childhood cancer and 115 family doctors of adult survivors of childhood cancer regarding shared care and found that 82 percent of participating local family doctors were satisfied with the shared care collaboration and thought the information they had received from the long-term followup clinic was adequate. ${ }^{55}$ In this study, all family doctors were provided information on the survivor's history, health risks, and required tests. ${ }^{55}$ Other studies have also noted the importance of regular communication and close collaboration between pediatric oncology and PCPs, as well as documents such as SCPs, so that PCPs are aware of and can address the unique health risks of childhood cancer survivors. ${ }^{48,51}$ However, there is some evidence that current levels of communication are inadequate. In a Swiss survey of providers, 94 percent of GPs reported wanting more support from oncologists regarding followup for childhood cancer survivors. ${ }^{57}$

Two studies from the literature related to adult survivors of adult cancer provide additional insights. In a description of a Primary Care for Cancer Survivor Clinic, Choi et al. report how the high quality of communication between the clinic and oncology providers is valued. ${ }^{67}$ Another study looked at the degree to which providers shared patients in a cohort of 8,661 cancer survivors and found that a greater degree of patient sharing among providers was associated with higher quality care on some measures and lower costs. ${ }^{68}$

When implemented effectively, shared-care models provide close connections and quality communication so that PCPs have the information they need from cancer specialists to deliver appropriate survivorship care.

\section{Context C: More Experience Caring for Childhood Cancer Survivors}

There is limited evidence suggesting that the more experience PCPs have caring for cancer survivors, the more comfortable they feel doing so and the better they adhere to guidelines. In a survey of 1,110 internists, those who saw at least one childhood cancer survivor in the last 5 years reported being more comfortable seeing childhood cancer survivors and were more likely to promote appropriate breast cancer surveillance. ${ }^{26}$ Findings in a survey of 1,024 family practitioners were similar. ${ }^{25}$ Family physicians who had seen at least one cancer survivor in the past 5 years reported more comfort in doing so and were more likely to correctly identify appropriate surveillance strategies.

However, most PCPs treat few if any childhood cancer survivors. Based on data from two surveys, only 51 percent of internists and 58 percent of family practitioners report having cared for one or more childhood cancer survivors in the past 5 years. ${ }^{25,}{ }^{26}$ In another survey of PCPs, 40 
percent reported they had never cared for one. ${ }^{56}$ Among the 134 PCPs in the Iyer et al. study, the average number of childhood cancer survivors cared for in the past 5 years was one, with 84 percent reporting having cared for only one. ${ }^{59}$ They also had little exposure to late effects, with only 34 percent having seen at least 5 late effects and 45 percent reporting seeing at least one late effect of grade 3 or higher. In Switzerland, Michel et al. discuss the possibility of having one specialized PCP for a certain catchment area, although the possibility of translating this idea to the U.S. context is unclear. ${ }^{57}$

These themes were echoed in a study of 86 PCPs regarding care for adult survivors of hematologic malignancies and hematopoietic cell transplantation. ${ }^{69}$ PCPs who had cared for more survivors felt more confident and perceived fewer barriers to doing so. They also were more likely to report that they discussed screening and late effects with patients.

While there is some evidence that having a greater concentration of childhood cancer survivors in a PCP's practice could promote greater provider comfort and lead to more appropriate care, opportunities to operationalize this approach in the U.S. may be limited. 


\section{Discussion}

\section{Summary of Findings}

This realist review addresses how models of survivorship care that include primary care, and resources for adult survivors of childhood cancer and their families and providers, can be effective. Undergirding this review were Contextual Questions (CQs) that:

- Define effectiveness for the models and resources;

- Describe models of care, highlighting those that include primary care; and

- Identify resources directed to childhood cancer survivors and their families and to care providers.

Effectiveness for both resources and models is defined by survivors living longer and feeling better through high-value care. Intermediate measures of effectiveness evaluate the extent to which:

- Survivors feel confident about sharing their history, know their risks, recognize symptoms and problems, understand the care they need, are aware of the resources available to help them, and can access relevant care and services; and

- Primary care providers (PCPs) understand a survivor's history, know the survivor's risks, recognize symptoms and problems, understand the care survivors need, are aware of the resources available to help them, and can access relevant care and services.

Specific outcomes that are assessed include:

- Loss to followup;

- Surveillance for long-term and late effects of original cancer treatment;

- Concordance with guidelines;

- Communication, transfer of information, and care coordination;

- Morbidity and quality of life;

- Mortality; and

- Costs.

In terms of models of care, we noted that the taxonomies such as that proposed by the American Society of Clinical Oncologists (ASCO) do not necessarily reflect real-world practice, nor the data presented in research studies. Both research and practice more likely reflect patterns of care that occur not by design but owing to circumstance. We therefore proposed four differentiating factors to define models of care:

1. The inclusion of survivorship expertise (whether via a specialized PCP or oncologist; an MD, nurse practitioner/physician's assistant, or multidisciplinary team);

2. The role of the PCP (e.g., main provider of survivorship care, provides survivorship care under the guidance of survivorship expert, provides primary care with no particular attention to survivorship);

3. Degree of access to academic/cancer center support for survivors and/or providers; and

4. Delivery of consultative versus longitudinal care.

For the purposes of this project, the term "resources" included:

- Long-term followup guidelines; 
- Educational materials;

- Trainings;

- Survivor care documents (e.g., survivorship care plans);

- Survivorship care management processes (i.e., expedited routes of contact for consultation); and

- Survivor supportive tools and services (e.g., support groups).

We identified 40 resources freely available to both survivors and providers: 23 survivorspecific, 12 provider/researcher-specific, 5 for both. We also identified 15 guidelines. Resources are most helpful if they are

- Easy to access,

- User-friendly,

- Known to survivors and providers,

- From trustworthy sources, and

- Valued by survivors and their families.

With the answers to these CQs as a foundation, we explored how models of care that include primary care, as well as resources, could be effective for adult survivors of childhood cancer. To begin to understand the relationships among the factors related to childhood cancer survivorship care, we developed an initial program theory and then refined it. This program theory describes how, nested in the overall environment, survivor and provider characteristics and facilitators/barriers interact to produce intermediate and then final outcomes. As shown in Figure 3 , the models of care and resources intersect with these factors, leading to survivors' and PCPs' ability to obtain/deliver appropriate care as the intermediate outcome, and survivors living longer and feeling better through high-value care as the final outcome.

Building on this program theory, to address the realist review KQs (KQs 1 and 2), we developed seven context-mechanism-outcome (CMO) hypotheses, four focused on the survivor intermediate outcome and three focused on the provider intermediate outcome. Because the models of care that include primary care (Key Question [KQ] 1) and resources (KQ2) were closely linked, we addressed these realist review KQs jointly.

For the CMO hypotheses for survivors, we hypothesized that four mechanisms would be associated with higher levels of the survivor intermediate outcome:

1. Linking resources with information that survivors can themselves use and also share with their PCP,

2. Identifying perceived/actually healthier survivors who have perceived/actually lower needs for survivorship-specific care,

3. Connecting survivors' engagement in the health system with increased knowledge about survivorship care, and

4. Suggesting that survivors with greater confidence in their PCPs would be more willing to transition their care.

For the CMO hypotheses for PCPs, we hypothesized that three mechanisms would be associated with higher levels of the provider intermediate outcome:

1. Linking resources with information to guide the PCP in delivering survivorship care,

2. Identifying the shared care model as a way to obtain the needed support from oncologists, and 
3. Suggesting that PCPs with more experience caring for childhood cancer survivors would have greater comfort and expertise in doing so.

As described in the intermediate outcomes, at the most basic level, the models of care and the resources seek to provide information to survivors and/or PCPs to enable them to obtain/deliver appropriate care. Thus, it is unsurprising that the variables from our program theory that were seen most consistently in the literature include oncology care versus primary care, survivor and provider knowledge, provider comfort in treating childhood cancer survivors, communication and coordination between and among providers and survivors, and delivery/receipt of prevention and surveillance of late effects of the original cancer treatment. In turn, these were the variables that played the most prominent role in our CMO hypotheses.

Notably, our discussion of the CMO hypotheses also describe why they may not be effective in achieving the desired outcomes. For example, we hypothesize that information from resources is a key mechanism for achieving the intermediate and final outcomes for both survivors and providers. However, for survivors, we also discuss the evidence regarding how the information may be both beneficial and harmful, how the information may be more effective for some populations than others (e.g., survivors with lower baseline knowledge may benefit more; female survivors may engage with resources more), and the challenges of delivering the appropriate "dose" of information to be effective. Similarly, for providers, we note that their lack of awareness of resources, the possibilities of and problems with information in the electronic health record (her), and, again, balancing the "dose" of information so that it is useful without requiring undue burden. In this way, our CMOs describe both how various mechanisms could be effective, as well as why they may not be.

In combination, the answers to the CQs, the program theory, and the CMO hypotheses provide valuable insights into the how and for whom, in what contexts, and via what mechanisms models of care that include primary care and resources could be effective for adult survivors of childhood cancer. It therefore addresses its purpose of informing future research investment by the National Cancer Institute. More generally, this review can inform future research and health system planning to promote improved care and outcomes for this important patient population.

\section{Strengths and Limitations}

It is important to consider these findings in the context of the strengths and limitations of this realist review. First, we were charged with answering the question of how models of survivorship care that include primary care could be effective - not whether they are effective. While not the focus of this review, we did find evidence that oncology specialty care is more effective in providing survivorship care for childhood cancer survivors. ${ }^{7,9,10,25,26,31,48,54,57,59,70-}$ 72 This review focused specifically on survivorship care models, not the overall care of adult survivors of childhood cancer (e.g., unrelated comorbid conditions, general preventive care) where the role of the primary care provider is more clear.

It is also worth noting that, while this realist review aimed to address "models of care," as described in the CQs, specific models of care are not clearly delineated and are rarely purposefully selected. Rather, the literature generally only provides evidence regarding "patterns of care" (i.e., who got seen where and received what), not evaluations of formal models of care. This limitation of the literature further complicated our realist review, which would ideally focus on a "well-defined program." ${ }^{, 73}$ In summary, rather than conducting a realist review of one well- 
defined model of care, we faced the challenge of conducting a realist review of multiple illdefined patterns of care.

Strengths of our literature review and abstraction approach include the investigation of a wide range of papers, in multiple contexts, internationally. For the development of the initial program theory, we focused on commentaries, editorials, and qualitative and mixed-methods papers that described intended operations and outcomes of models of care and resources. During refinement of the program theory, we focused on quantitative studies that could inform our program theory revisions and $\mathrm{CMO}$ hypothesis development. We not only included studies examining models of care and resources for adult survivors of childhood cancer, but also for child/adolescent survivors, as well as adult survivors of adult cancer. While the data from other populations added insights informing our CMOs, the generalizability of these findings to adult survivors of childhood cancer requires further exploration. We did not restrict our literature review to studies in the U.S., although the applicability of studies from other countries with different health systems may be limited. Given the limited time available to conduct the literature review and abstraction, we could not go through as many iterations of developing theories and conducting additional searches to explore these theories further, as has been done in some other realist reviews. Rather, we largely conducted the review and abstraction in one large effort and then prioritized which studies we focused on in developing our CMO hypotheses. Specifically, our CMOs were developed primarily based on the literature for adult/adolescent/childhood survivors of childhood cancer, although we drew on the literature from adult survivors of adult cancer where it added explanatory value. With realist reviews, the goal of the literature abstraction is to be informative and illustrative, rather than comprehensive and confirmatory.

A final strength of our realist review is the multidisciplinary expertise and extensive experience of our research team and Stakeholders. The research team included expertise in childhood cancer survivorship clinical care, survivorship research, oncology care, primary care, health services research, qualitative methods, and systematic reviews. We also received valuable input from our Stakeholders. Eight individuals provided a range of expertise and experience in childhood cancer survivorship research, advocacy, and clinical care; one expert advised on realist review methods. We consulted with these Stakeholders at three timepoints during this process: at project initiation, after developing the first draft of the initial program theory, and after revising the program theory and developing the CMO hypotheses.

\section{Gaps in the Literature and Future Directions}

Several gaps in the literature are noteworthy and should inform future directions in both qualitative and quantitative research in this area. First, there is a lack of formal evaluations of models of care, particularly models that include primary care. As described above, the lack of a practical taxonomy of models of care contributes to this problem, with the ASCO taxonomy reflecting more theoretical models as opposed to the frequently unplanned patterns of care that is experienced by survivors and studied in the literature. In addition, data regarding final outcomes, particularly mortality, are sparse (e.g., are survivors who are more adherent to recommended surveillance more likely to live longer?). Other outcomes that were examined less frequently in the literature include impact of models on resource utilization (e.g., emergency department visits, hospitalizations) and costs. Other more specific issues mentioned in the Results, such as the possible need to tailor resources to different groups (e.g., males vs. females), should also be explored. 
During the conduct of this review, the world experienced the transforming effects of the COVID-19 global pandemic. This factor is reflected in our program theory variable list as "crisis events," but the literature has not even begun to reflect how medical care in general, and cancer survivorship care in particular, may be changed. For example, where the use of telemedicine was relatively limited in 2019, it became commonplace - and in some cases dominant, in $2020{ }^{74}$

As described by one of our Stakeholders, the pandemic further emphasized two research questions related to this review: (1) who needs to be seen in specialty care and who can be followed in their own community; and (2) for those followed in the community, how can the knowledge that survivors and PCPs need to receive/deliver quality care be effectively transferred? These questions represent the crux of the issues that require further research.

\section{Conclusion}

In summary, care for adult survivors of childhood cancer is complex, and the appropriate models for delivering this care are unclear. In fact, the various models of care are not welldefined, and what is seen in the literature (and experienced by survivors) are more often patterns of care that occur not by design but owing to circumstance.

While there is evidence that suggests that care delivered in a specialty setting is superior, various barriers outlined in our program theory (e.g., availability of specialized care, distance to care) describe why it may not be universally available. Our charge in conducting this realist review was to describe for whom and under what circumstances models of care that include primary care could be effective for adult survivors of childhood cancer. A common theme across the CMO hypotheses developed as part of this realist review is that, if care is going to be delivered outside of the specialty setting, there has to be knowledge transfer to survivors and PCPs.

This realist review identified a number of ways this knowledge could be shared, including a range of resources (e.g., guidelines, survivorship care plans [SCPs]) and contexts (e.g., survivor confidence in PCPs, shared care with oncologists). The resulting program theory and related hypotheses elucidate some of the key CMO relationships that could be associated with effective survivorship care models that include primary care. Further research is required to explore whether these CMO relationships can be effectively actualized. 


\section{References}

1. Cancer Treatment \& Survivorship Facts \& Figures 2019-2021 American Cancer Society. Atlanta, GA: 2019.

2. Hudson MM, Ness KK, Gurney JG, et al. Clinical ascertainment of health outcomes among adults treated for childhood cancer. Jama. 2013 Jun 12;309(22):2371-81. doi: 10.1001/jama.2013.6296. PMID: 23757085.

3. Bhakta N, Liu Q, Ness KK, et al. The cumulative burden of surviving childhood cancer: an initial report from the St Jude Lifetime Cohort Study (SJLIFE). Lancet (London, England). 2017 Dec 9;390(10112):2569-82. doi: 10.1016/s0140-6736(17)31610-0. PMID: 28890157.

4. Armstrong GT, Kawashima T, Leisenring W, et al. Aging and risk of severe, disabling, lifethreatening, and fatal events in the childhood cancer survivor study. Journal of clinical oncology : official journal of the American Society of Clinical Oncology. 2014 Apr 20;32(12):1218-27. doi: 10.1200/jco.2013.51.1055. PMID: 24638000 .

5. Meacham LR, Chow EJ, Ness KK, et al. Cardiovascular risk factors in adult survivors of pediatric cancer--a report from the childhood cancer survivor study. Cancer epidemiology, biomarkers \& prevention : a publication of the American Association for Cancer Research, cosponsored by the American Society of Preventive Oncology. 2010 Jan;19(1):170-81. doi: 10.1158/10559965.epi-09-0555. PMID: 20056636.

6. Geenen MM, Cardous-Ubbink MC, Kremer LC, et al. Medical assessment of adverse health outcomes in long-term survivors of childhood cancer. Jama. 2007 Jun 27;297(24):2705-15. doi: 10.1001/jama.297.24.2705. PMID: 17595271.
7. Nathan PC, Greenberg ML, Ness KK, et al. Medical care in long-term survivors of childhood cancer: a report from the childhood cancer survivor study. Journal of clinical oncology : official journal of the American Society of Clinical Oncology. 2008 Sep 20;26(27):4401-9. doi: 10.1200/jco.2008.16.9607. PMID: 18802152 .

8. Institute of M, National Research Council National Cancer Policy B. In: Hewitt M, Weiner SL, Simone JV, eds. Childhood Cancer Survivorship: Improving Care and Quality of Life. Washington (DC): National Academies Press (US)

Copyright 2003 by the National Academy of Sciences. All rights reserved.; 2003.

9. Hudson MM. A model for care across the cancer continuum. Cancer. 2005 Dec 1;104(11 Suppl):2638-42. doi: 10.1002/cncr.21250. PMID: 16258932.

10. Nathan PC, Ford JS, Henderson TO, et al. Health behaviors, medical care, and interventions to promote healthy living in the Childhood Cancer Survivor Study cohort. Journal of clinical oncology : official journal of the American Society of Clinical Oncology. 2009 May 10;27(14):2363-73. doi: 10.1200/jco.2008.21.1441. PMID: 19255308 .

11. Pawson R, Greenhalgh T, Harvey G, et al. Realist review--a new method of systematic review designed for complex policy interventions. Journal of health services research \& policy. 2005 Jul;10 Suppl 1:21-34. doi: 10.1258/1355819054308530. PMID: 16053581.

12. The RAMESES Projects: Standards and Training Materials. 2020. https://ramesesproject.org/Standards and $\mathrm{Tr}$ aining_materials.php\#rs_training. Accessed on June 102020. 
13. Abrams R, Wong G, Mahtani KR, et al. Understanding the impact of delegated home visiting services accessed via general practice by community-dwelling patients: a realist review protocol. BMJ open. 2018 Nov 13;8(11):e024876. doi: 10.1136/bmjopen-2018-024876. PMID: 30429150 .

14. Greenhalgh J, Pawson R, Wright J, et al. Functionality and feedback: a protocol for a realist synthesis of the collation, interpretation and utilisation of PROMs data to improve patient care. BMJ open. $2014 \mathrm{Jul}$ 22;4(7):e005601. doi: 10.1136/bmjopen2014-005601. PMID: 25052175.

15. Booth A, Briscoe S, Wright JM. The "realist search": A systematic scoping review of current practice and reporting. Research synthesis methods. 2020 Jan;11(1):14-35. doi: 10.1002/jrsm.1386. PMID: 31714016.

16. Supplemental Evidence and Data Request on Models of Care That Include Primary Care for Adult Survivors of Childhood Cancer. Federal Register; 2020. https://www.govinfo.gov/content/pkg/FR2020-11-25/pdf/2020-26041.pdf. Accessed on May 2021.

17. Booth A, Harris J, Croot E, et al. Towards a methodology for cluster searching to provide conceptual and contextual "richness" for systematic reviews of complex interventions: case study (CLUSTER). BMC medical research methodology. 2013 Sep 28;13:118. doi: 10.1186/1471-2288-13-118. PMID: 24073615.

18. Doing Realist Research. 2020/06/17. 55 City Road, London: SAGE Publications Ltd. 2018. Available from: https://methods.sagepub.com/book/doingrealist-research. 10.4135/9781526451729.

19. Carrieri D, Briscoe S, Jackson M, et al. 'Care Under Pressure': a realist review of interventions to tackle doctors' mental illhealth and its impacts on the clinical workforce and patient care. BMJ open. 2018 Feb 2;8(2):e021273. doi: 10.1136/bmjopen2017-021273. PMID: 29420234.
20. CASP. Qualitative Research Checklist. Secondary Qualitative Research Checklist. 2013.

http://media.wix.com/ugd/dded87_29c5b002 d99342f788c6ac670e49f274.pdf.

21. American Society of Clinical Oncology. Models of long-term follow-up care. 2020. https://www.asco.org/practicepolicy/cancer-careinitiatives/preventionsurvivorship/survivorsh ip/survivorship-3. Accessed on June 10 2020.

22. Oeffinger KC, Mertens AC, Hudson MM, et al. Health care of young adult survivors of childhood cancer: a report from the Childhood Cancer Survivor Study. Annals of family medicine. $2004 \mathrm{Jan}-\mathrm{Feb}$;2(1):6170. doi: 10.1370/afm.26. PMID: 15053285.

23. Mueller EL, Park ER, Kirchhoff AC, et al. Insurance, chronic health conditions, and utilization of primary and specialty outpatient services: a Childhood Cancer Survivor Study report. Journal of cancer survivorship : research and practice. 2018 Oct;12(5):639-46. doi: 10.1007/s11764-0180700-1. PMID: 29943170.

24. Henderson TO, Hlubocky FJ, Wroblewski KE, et al. Physician preferences and knowledge gaps regarding the care of childhood cancer survivors: a mailed survey of pediatric oncologists. Journal of clinical oncology : official journal of the American Society of Clinical Oncology. 2010 Feb 10;28(5):87883. doi: 10.1200/jco.2009.25.6107. PMID: 20038717.

25. Nathan PC, Daugherty CK, Wroblewski KE, et al. Family physician preferences and knowledge gaps regarding the care of adolescent and young adult survivors of childhood cancer. Journal of cancer survivorship : research and practice. 2013 Sep;7(3):275-82. doi: 10.1007/s11764-0130271-0. PMID: 23471729.

26. Suh E, Daugherty CK, Wroblewski K, et al. General internists' preferences and knowledge about the care of adult survivors of childhood cancer: a cross-sectional survey. Annals of internal medicine. 2014 Jan 7;160(1):11-7. doi: 10.7326/m13-1941. PMID: 24573662. 
27. Singer S, Gianinazzi ME, Hohn A, et al. General practitioner involvement in follow-up of childhood cancer survivors: a systematic review. Pediatric blood \& cancer. 2013 Oct;60(10):1565-73. doi: 10.1002/pbc.24586. PMID: 23813795.

28. Andersen RM. Revisiting the behavioral model and access to medical care: does it matter? Journal of health and social behavior. 1995 Mar;36(1):1-10. PMID: 7738325.

29. Shay LA, Parsons HM, Vernon SW. Survivorship Care Planning and Unmet Information and Service Needs Among Adolescent and Young Adult Cancer Survivors. Journal of adolescent and young adult oncology. 2017 Jun;6(2):327-32. doi: 10.1089/jayao.2016.0053. PMID: 28103126.

30. Murphy P, Levine A, Lerma T, et al. A portable survivorship care plan: a tool that helps educate and improve knowledge in childhood cancer survivors. Supportive care in cancer : official journal of the Multinational Association of Supportive Care in Cancer. 2021 Jan;29(1):169-77. doi: 10.1007/s00520-020-05422-z. PMID: 32328773.

31. Yan AP, Chen Y, Henderson TO, et al. Adherence to Surveillance for Second Malignant Neoplasms and Cardiac Dysfunction in Childhood Cancer Survivors: A Childhood Cancer Survivor Study. Journal of Clinical Oncology. 2020;38(15):1711-22. doi: 10.1200/JCO.19.01825. PMID: 143226932. Language: English. Entry Date: In Process. Revision Date: 20201113. Publication Type: journal article. Journal Subset: Biomedical.

32. Spain PD, Oeffinger KC, Candela J, et al. Response to a treatment summary and care plan among adult survivors of pediatric and young adult cancer. Journal of oncology practice. 2012 May;8(3):196-202. doi: 10.1200/jop.2011.000345. PMID: 22942816.

33. Pannier ST, Mann K, Warner EL, et al. Survivorship care plan experiences among childhood acute lymphoblastic leukemia patients and their families. BMC pediatrics. 2019 Apr 13;19(1):111. doi: 10.1186/s12887-019-1464-0. PMID: 30979365 .
34. Oeffinger KC, Hudson MM, Mertens AC, et al. Increasing rates of breast cancer and cardiac surveillance among high-risk survivors of childhood Hodgkin lymphoma following a mailed, one-page survivorship care plan. Pediatric blood \& cancer. 2011 May;56(5):818-24. doi: 10.1002/pbc.22696. PMID: 21370417.

35. Steele JR, Wall M, Salkowski N, et al. Predictors of risk-based medical follow-up: a report from the Childhood Cancer Survivor Study. Journal of cancer survivorship : research and practice. $2013 \mathrm{Sep}$ 7(3):379-91. doi: 10.1007/s11764-013-0280-z. PMID: 23568405.

36. Nicolaije KA, Ezendam NP, Vos MC, et al. Impact of an Automatically Generated Cancer Survivorship Care Plan on PatientReported Outcomes in Routine Clinical Practice: Longitudinal Outcomes of a Pragmatic, Cluster Randomized Trial. Journal of clinical oncology : official journal of the American Society of Clinical Oncology. 2015 Nov 1;33(31):3550-9. doi: 10.1200/jco.2014.60.3399. PMID: 26304900.

37. de Rooij BH, Ezendam NPM, Nicolaije KAH, et al. Effects of Survivorship Care Plans on patient reported outcomes in ovarian cancer during 2-year follow-up - The ROGY care trial. Gynecologic oncology. 2017 May;145(2):319-28. doi: 10.1016/j.ygyno.2017.02.041. PMID: 28283195.

38. Jabson JM, Bowen DJ. How do follow-up care instructions and treatment summaries relate to cancer survivors' cancer-related pain? Journal of pain and symptom management. 2014 Dec;48(6):1247-53. doi: 10.1016/j.jpainsymman.2014.03.004. PMID: 24780182.

39. Jabson JM. Follow-up care instructions, treatment summaries, and cancer survivors' receipt of follow-up health care and late/long term effects. Supportive care in cancer : official journal of the Multinational Association of Supportive Care in Cancer. 2015 Jul;23(7):1851-6. doi: 10.1007/s00520-0142532-5. PMID: 25471181. 
40. de Rooij BH, Ezendam NPM, Vos MC, et al. Patients' information coping styles influence the benefit of a survivorship care plan in the ROGY Care Trial: New insights for tailored delivery. Cancer. 2019 Mar 1;125(5):78897. doi: 10.1002/cncr.31844. PMID: 30500067.

41. Landier W, Chen Y, Namdar G, et al. Impact of Tailored Education on Awareness of Personal Risk for Therapy-Related Complications Among Childhood Cancer Survivors. Journal of clinical oncology : official journal of the American Society of Clinical Oncology. 2015 Nov 20;33(33):3887-93. doi: 10.1200/jco.2015.62.7562. PMID: 26324371.

42. de Rooij BH, Park ER, Perez GK, et al. Cluster Analysis Demonstrates the Need to Individualize Care for Cancer Survivors. The oncologist. 2018 Dec;23(12):1474-81. doi: 10.1634/theoncologist.2017-0558. PMID: 29739897.

43. Tevaarwerk AJ, Hocking WG, Buhr KA, et al. A randomized trial of immediate versus delayed survivorship care plan receipt on patient satisfaction and knowledge of diagnosis and treatment. Cancer. 2019 Mar 15;125(6):1000-7. doi: 10.1002/cncr.31875. PMID: 30690714.

44. Hudson MM, Tyc VL, Srivastava DK, et al. Multi-component behavioral intervention to promote health protective behaviors in childhood cancer survivors: the protect study. Medical and pediatric oncology. 2002 Jul;39(1):2-1; discussion 2. doi: 10.1002/mpo.10071. PMID: 12116072.

45. Turner J, Yates P, Kenny L, et al. The ENHANCES study: a randomised controlled trial of a nurse-led survivorship intervention for patients treated for head and neck cancer. Supportive care in cancer : official journal of the Multinational Association of Supportive Care in Cancer. 2019 Dec;27(12):4627-37. doi: 10.1007/s00520019-04748-7. PMID: 30941580.
46. Casillas J, Oeffinger KC, Hudson MM, et al. Identifying Predictors of Longitudinal Decline in the Level of Medical Care Received by Adult Survivors of Childhood Cancer: A Report from the Childhood Cancer Survivor Study. Health services research. 2015 Aug;50(4):1021-42. doi: 10.1111/1475-6773.12282. PMID: 25600956.

47. Klosky JL, Cash DK, Buscemi J, et al. Factors influencing long-term follow-up clinic attendance among survivors of childhood cancer. Journal of cancer survivorship : research and practice. $2008 \mathrm{Dec}$;2(4):22532. doi: 10.1007/s11764-008-0063-0. PMID: 18787958.

48. Christen S, Vetsch J, Mader L, et al. Preferences for the organization of long-term follow-up in adolescent and young adult cancer survivors. Supportive care in cancer : official journal of the Multinational Association of Supportive Care in Cancer. 2016 Aug;24(8):3425-36. doi: 10.1007/s00520-016-3157-7. PMID: 26988228.

49. Signorelli C, Wakefield CE, Johnston KA, et al. Re-Engage: A Novel Nurse-Led Program for Survivors of Childhood Cancer Who Are Disengaged From Cancer-Related Care. Journal of the National Comprehensive Cancer Network : JNCCN. 2020 Aug;18(8):1067-74. doi: 10.6004/jncen.2020.7552. PMID: 32755982.

50. Guilcher GM, Fitzgerald C, Pritchard S. A questionnaire based review of long-term follow-up programs for survivors of childhood cancer in Canada. Pediatric blood \& cancer. 2009 Jan;52(1):113-5. doi: 10.1002/pbc.21701. PMID: 18821577.

51. Hudson MM, Leisenring W, Stratton KK, et al. Increasing cardiomyopathy screening in atrisk adult survivors of pediatric malignancies: a randomized controlled trial. Journal of clinical oncology : official journal of the American Society of Clinical Oncology. 2014 Dec 10;32(35):3974-81. doi: 10.1200/jco.2014.57.3493. PMID: 25366684. 
52. Berg CJ, Stratton E, Esiashvili N, et al. Young Adult Cancer Survivors' Experience with Cancer Treatment and Follow-Up Care and Perceptions of Barriers to Engaging in Recommended Care. Journal of cancer education : the official journal of the American Association for Cancer Education. 2016 Sep;31(3):430-42. doi: 10.1007/s13187-015-0853-9. PMID: 25948413.

53. Svedberg P, Einberg EL, Wärnestål P, et al. Support from healthcare services during transition to adulthood - Experiences of young adult survivors of pediatric cancer. European journal of oncology nursing : the official journal of European Oncology Nursing Society. 2016 Apr;21:105-12. doi: 10.1016/j.ejon.2016.02.008. PMID: 26952685 .

54. Signorelli C, Wakefield C, McLoone JK, et al. Childhood cancer survivorship: barriers and preferences. BMJ supportive \& palliative care. 2019 Nov 11. doi: 10.1136/bmjspcare2019-002001. PMID: 31712388.

55. Blaauwbroek R, Tuinier W, Meyboom-de Jong $\mathrm{B}$, et al. Shared care by paediatric oncologists and family doctors for long-term follow-up of adult childhood cancer survivors: a pilot study. The Lancet Oncology. 2008 Mar;9(3):232-8. doi: 10.1016/s1470-2045(08)70034-2. PMID: 18282804 .

56. Sima JL, Perkins SM, Haggstrom DA. Primary care physician perceptions of adult survivors of childhood cancer. Journal of pediatric hematology/oncology. 2014 Mar;36(2):11824. doi: $10.1097 / \mathrm{mph} .0000000000000061$. PMID: 24309612.

57. Michel G, Gianinazzi ME, Vetsch J, et al. Physicians' experience with follow-up care of childhood cancer survivors - challenges and needs. Swiss medical weekly.

2017;147:w14457. doi:

10.4414/smw.2017.14457. PMID: 28722079 .

58. Blaauwbroek R, Zwart N, Bouma M, et al. The willingness of general practitioners to be involved in the follow-up of adult survivors of childhood cancer. Journal of cancer survivorship : research and practice. 2007 Dec;1(4):292-7. doi: 10.1007/s11764-0070032-z. PMID: 18648964.
59. Iyer NS, Mitchell HR, Zheng DJ, et al. Experiences with the survivorship care plan in primary care providers of childhood cancer survivors: a mixed methods approach. Supportive care in cancer : official journal of the Multinational Association of Supportive Care in Cancer. 2017 May;25(5):1547-55. doi: 10.1007/s00520-016-3544-0. PMID: 28050709.

60. Donohue S, Sesto ME, Hahn DL, et al. Evaluating primary care providers' views on survivorship care plans generated by an electronic health record system. Journal of oncology practice. 2015 May;11(3):e329-35. doi: 10.1200/jop.2014.003335. PMID: 25804989.

61. Campbell BH, Massey BL, Myers KB. Survivorship care plans for patients with head and neck cancer. Archives of otolaryngology--head \& neck surgery. 2012 Dec;138(12):1116-9. doi: 10.1001/jamaoto.2013.683. PMID: 23247230 .

62. Nicolaije KA, Ezendam NP, Vos MC, et al. Oncology providers' evaluation of the use of an automatically generated cancer survivorship care plan: longitudinal results from the ROGY Care trial. Journal of cancer survivorship : research and practice. 2014 Jun;8(2):248-59. doi: 10.1007/s11764-0130327-1. PMID: 24357226.

63. Morken CM, Tevaarwerk AJ, Swiecichowski AK, et al. Survivor and Clinician Assessment of Survivorship Care Plans for Hematopoietic Stem Cell Transplantation Patients: An Engineering, Primary Care, and Oncology Collaborative for Survivorship Health. Biology of blood and marrow transplantation : journal of the American Society for Blood and Marrow Transplantation. 2019 Jun;25(6):1240-6. doi: 10.1016/j.bbmt.2019.02.003. PMID: 30763727.

64. Birken SA, Mayer DK, Weiner BJ. Survivorship care plans: prevalence and barriers to use. Journal of cancer education : the official journal of the American Association for Cancer Education. 2013 Jun;28(2):290-6. doi: 10.1007/s13187-013-0469-x. PMID: 23526552 . 
65. Birken SA, Raskin S, Zhang Y, et al. Survivorship care plan implementation in US cancer programs: A national survey of cancer care providers. Journal of Cancer Education. 2019;34(3):614-22. doi: 10.1007/s13187-018-1374-0. PMID: 201936497-028.

66. Costello AG, Nugent BD, Conover N, et al. Shared Care of Childhood Cancer Survivors: A Telemedicine Feasibility Study. Journal of Adolescent \& Young Adult Oncology. 2017;6(4):535-41. doi: 10.1089/jayao.2017.0013. PMID: 126600837. Language: English. Entry Date: 20171213. Revision Date: 20181203. Publication Type: Article.

67. Choi Y, Radhakrishnan A, Mahabare D, et al. The Johns Hopkins Primary Care for Cancer Survivor Clinic: lessons learned in our first 4 years. Journal of cancer survivorship : research and practice. $2020 \mathrm{Feb} ; 14(1): 19-25$. doi: 10.1007/s11764-019-00816-3. PMID: 31650473 .

68. Pollack CE, Frick KD, Herbert RJ, et al. It's who you know: patient-sharing, quality, and costs of cancer survivorship care. Journal of cancer survivorship : research and practice. 2014 Jun;8(2):156-66. doi: 10.1007/s11764014-0349-3. PMID: 24578154.

69. Mani S, Khera N, Rybicki L, et al. Primary Care Physician Perspectives on Caring for Adult Survivors of Hematologic Malignancies and Hematopoietic Cell Transplantation. Clinical lymphoma, myeloma \& leukemia. 2020 Feb;20(2):70-7. doi: 10.1016/j.clml.2019.11.008. PMID: 31810888 .
70. Kadan-Lottick NS, Ross WL, Mitchell HR, et al. Randomized Trial of the Impact of Empowering Childhood Cancer Survivors With Survivorship Care Plans. Journal of the National Cancer Institute. $2018 \mathrm{Dec}$ 1;110(12):1352-9. doi: 10.1093/jnci/djy057. PMID: 29771337.

71. Sutradhar R, Agha M, Pole JD, et al. Specialized survivor clinic attendance is associated with decreased rates of emergency department visits in adult survivors of childhood cancer. Cancer. 2015 Dec 15;121(24):4389-97. doi: 10.1002/cncr.29679. PMID: 26406998.

72. Marr KC, Agha M, Sutradhar R, et al. Specialized survivor clinic attendance increases adherence to cardiomyopathy screening guidelines in adult survivors of childhood cancer. Journal of cancer survivorship : research and practice. 2017 Oct;11(5):614-23. doi: 10.1007/s11764-0170634-z. PMID: 28785871.

73. Shaw J, Gray CS, Baker GR, et al. Mechanisms, contexts and points of contention: operationalizing realist-informed research for complex health interventions. BMC medical research methodology. $2018 \mathrm{Dec}$ 27;18(1):178. doi: 10.1186/s12874-0180641-4. PMID: 30587138.

74. Weiner JP, Bandeian S, Hatef E, et al. In-Person and Telehealth Ambulatory Contacts and Costs in a Large US Insured Cohort Before and During the COVID-19 Pandemic. JAMA network open. 2021 Mar 1;4(3):e212618. doi: 10.1001/jamanetworkopen.2021.2618. PMID: 33755167. 


\section{Abbreviations and Acronyms}

\begin{tabular}{|l|l|}
\hline Acronym & Definition \\
\hline AHRQ & Agency for Healthcare Research and Quality \\
\hline ASCO & American Society of Clinical Oncology \\
\hline AYA & adolescent/young adult \\
\hline CASP & Critical Appraisal Skills Programme \\
\hline CMO & context-mechanism-outcome hypotheses \\
\hline COG & Children's Oncology Group \\
\hline CQ & Contextual Question \\
\hline EHR & electronic health record \\
\hline EPC & Evidence-based Practice Center \\
\hline GP & general practitioner \\
\hline KQ & Key Question \\
\hline MD & medical doctor \\
\hline NAM & National Academy of Medicine \\
\hline NCI & National Cancer Institute \\
\hline NP & nurse practitioner \\
\hline PA & physician's assistant \\
\hline PCP & primary care provider \\
\hline RAMESES & Realist And Meta-narrative Evidence Syntheses: Evolving Standards \\
\hline RCT & randomized controlled trial \\
\hline SCP & survivorship care plan \\
\hline STAR & The Childhood Cancer Survivorship, Treatment, Access, and Research (STAR) Act \\
\hline TOO & Task Order Officer \\
\hline
\end{tabular}




\section{Appendix A. Search Strategies}

Table A-1. PubMed search strategies

\begin{tabular}{|c|c|}
\hline \# & Term \\
\hline 1 & "Cancer Survivors"[Mesh] \\
\hline 2 & Cancer[tiab] \\
\hline 3 & survivorship[Mesh] \\
\hline 4 & Survivors[mh] \\
\hline 5 & survivorship[tiab] \\
\hline 6 & survivors[tiab] \\
\hline 7 & survivor[tiab] \\
\hline 8 & 1 OR 2 AND (3 OR 4 OR 5 OR 6 OR 7)) \\
\hline 9 & "health planning"[tiab] \\
\hline 10 & "health care planning"[tiab] \\
\hline 11 & "patient care planning"[tiab] \\
\hline 12 & "patient care bundles"[tiab] \\
\hline 13 & "patient care bundle"[tiab] \\
\hline 14 & "patient care documents"[tiab] \\
\hline 15 & "patient care document"[tiab] \\
\hline 16 & "patient care guidelines"[tiab] \\
\hline 17 & "educational materials"[tiab] \\
\hline 18 & "education materials"[tiab] \\
\hline 19 & Workshop[tiab] \\
\hline 20 & Workshops[tiab] \\
\hline 21 & "training program"[tiab] \\
\hline 22 & "training programs"[tiab] \\
\hline 23 & "management processes"[tiab] \\
\hline 24 & "management process"[tiab] \\
\hline 25 & "management plans"[tiab] \\
\hline 26 & "management plan"[tiab] \\
\hline 27 & "care plan"[tiab] \\
\hline 28 & "care plans"[tiab] \\
\hline 29 & "care model"[tiab] \\
\hline 30 & "Care models"[tiab] \\
\hline 31 & "Health Planning Guidelines"[Mesh] \\
\hline 32 & "Patient Care Planning"[Mesh] \\
\hline 33 & "Patient Care Bundles"[Mesh] \\
\hline 34 & $\begin{array}{l}9 \text { OR } 10 \text { OR } 11 \text { OR } 12 \text { OR } 13 \text { OR } 14 \text { OR } 15 \text { OR } 16 \text { OR } 17 \text { OR } 18 \text { OR } 19 \text { OR } 20 \text { OR } 21 \text { OR } 22 \text { OR } 23 \text { OR } \\
24 \text { OR } 24 \text { OR } 25 \text { OR } 26 \text { OR } 27 \text { OR } 28 \text { OR } 29 \text { OR } 30 \text { OR } 31 \text { OR } 32 \text { OR } 33\end{array}$ \\
\hline 35 & "Randomized Controlled Trial" [Publication Type] \\
\hline 36 & "Controlled Clinical Trial" [Publication Type] \\
\hline 37 & randomized[Title/Abstract] \\
\hline 38 & placebo[Title/Abstract] \\
\hline 39 & "drug therapy" [Subheading] \\
\hline 40 & randomly[Title/Abstract] \\
\hline 41 & trial[Title/Abstract] \\
\hline 42 & groups[Title/Abstract] \\
\hline 43 & $\begin{array}{l}\text { ("cross over" OR "crossover" OR "Follow Up") OR ("Cross-Over Studies"[MeSH] OR "Follow-Up } \\
\text { Studies"[MeSH] OR "Prospective Studies"[MeSH]) OR ("time series" OR "interrupted time series") OR } \\
\text { ("Case-Control Studies"[MeSH] OR (cases[Title/Abstract] AND controls[Title/Abstract])) OR ("Cohort } \\
\text { Studies"[MeSH] OR cohort*) OR ("Comparative Study"[Publication Type]) OR ("before after"[Title/Abstract] } \\
\text { OR "before-after"[Title/Abstract] OR "before/after"[Title/Abstract] OR "before and after"[Title/Abstract]) OR } \\
\text { (volunteer*[Title/Abstract]) OR (control*[Text Word] AND evaluation[Text Word]) OR (longitudinal[Text } \\
\text { Word]) OR (retrospective*[Text Word]) }\end{array}$ \\
\hline 45 & 35 OR 36 OR 37 OR 38 OR 39 OR 40 OR 41 OR 42 OR 43 \\
\hline 46 & 8 AND 34 AND 43 \\
\hline
\end{tabular}


Table A-2. APA Psyclnfo and CINAHL search strategies

\begin{tabular}{|c|c|c|}
\hline $\begin{array}{l}\text { Search } \\
\text { ID\# }\end{array}$ & Search Terms & Search Options \\
\hline S55 & S9 AND S34 AND S54 & $\begin{array}{l}\text { Expanders - Apply equivalent subjects } \\
\text { Search modes - Boolean/Phrase }\end{array}$ \\
\hline S54 & $\begin{array}{l}\text { S35 OR S36 OR S37 OR S38 OR S39 OR S40 OR } \\
\text { S41 OR S42 OR S43 OR S44 OR S45 OR S46 OR } \\
\text { S47 OR S48 OR S49 OR S50 OR S51 OR S52 OR } \\
\text { S53 }\end{array}$ & $\begin{array}{l}\text { Expanders - Apply equivalent subjects } \\
\text { Search modes - Boolean/Phrase }\end{array}$ \\
\hline S53 & TI retrospective OR AB retrospective & $\begin{array}{l}\text { Expanders - Apply equivalent subjects } \\
\text { Search modes - Boolean/Phrase }\end{array}$ \\
\hline S52 & TI longitudinal OR AB longitudinal & $\begin{array}{l}\text { Expanders - Apply equivalent subjects } \\
\text { Search modes - Boolean/Phrase }\end{array}$ \\
\hline S51 & $\begin{array}{l}\text { TI "before after" OR AB "before after" OR TI } \\
\text { "before-after" OR AB "before-after" OR TI ( "before } \\
\text { and after" ) OR AB ( "before and after" ) }\end{array}$ & $\begin{array}{l}\text { Expanders - Apply equivalent subjects } \\
\text { Search modes - Boolean/Phrase }\end{array}$ \\
\hline S50 & $\begin{array}{l}\text { TI "comparative study" OR AB "comparative study" } \\
\text { OR TI "comparative studies" OR AB "comparative } \\
\text { studies" }\end{array}$ & $\begin{array}{l}\text { Expanders - Apply equivalent subjects } \\
\text { Search modes - Boolean/Phrase }\end{array}$ \\
\hline S49 & $\begin{array}{l}\text { TI "cohort study" OR AB "cohort study" OR TI } \\
\text { "cohort studies" OR AB "cohort studies" }\end{array}$ & $\begin{array}{l}\text { Expanders - Apply equivalent subjects } \\
\text { Search modes - Boolean/Phrase }\end{array}$ \\
\hline S48 & $\begin{array}{l}\text { TI "case-control study" OR AB "case-control study" } \\
\text { AND TI "case-control studies" OR AB "case-control } \\
\text { studies" }\end{array}$ & $\begin{array}{l}\text { Expanders - Apply equivalent subjects } \\
\text { Search modes - Boolean/Phrase }\end{array}$ \\
\hline S47 & $\begin{array}{l}\text { TI "case controlled study" OR AB "case controlled } \\
\text { study" OR TI "case controlled studies" OR AB "case } \\
\text { controlled studies" }\end{array}$ & $\begin{array}{l}\text { Expanders - Apply equivalent subjects } \\
\text { Search modes - Boolean/Phrase }\end{array}$ \\
\hline S46 & TI "time series" OR AB Time series" & $\begin{array}{l}\text { Expanders - Apply equivalent subjects } \\
\text { Search modes - Boolean/Phrase }\end{array}$ \\
\hline S45 & $\begin{array}{l}\text { TI "Prospective study" OR AB "Prospective study" } \\
\text { OR TI "Prospective studies" OR AB "Prospective } \\
\text { studies" }\end{array}$ & $\begin{array}{l}\text { Expanders - Apply equivalent subjects } \\
\text { Search modes - Boolean/Phrase }\end{array}$ \\
\hline S44 & $\begin{array}{l}\text { TI "follow up" OR AB "follow up" OR TI "follow-up" } \\
\text { OR AB "follow-up" }\end{array}$ & $\begin{array}{l}\text { Expanders - Apply equivalent subjects } \\
\text { Search modes - Boolean/Phrase }\end{array}$ \\
\hline S43 & $\begin{array}{l}\text { TI "cross over" OR AB "cross over" OR TI crossover } \\
\text { OR AB crossover }\end{array}$ & $\begin{array}{l}\text { Expanders - Apply equivalent subjects } \\
\text { Search modes - Boolean/Phrase }\end{array}$ \\
\hline S42 & TI study OR AB study & $\begin{array}{l}\text { Expanders - Apply equivalent subjects } \\
\text { Search modes - Boolean/Phrase }\end{array}$ \\
\hline S41 & TI groups OR AB groups & $\begin{array}{l}\text { Expanders - Apply equivalent subjects } \\
\text { Search modes - Boolean/Phrase }\end{array}$ \\
\hline S40 & TI trial OR AB trial & $\begin{array}{l}\text { Expanders - Apply equivalent subjects } \\
\text { Search modes - Boolean/Phrase }\end{array}$ \\
\hline S39 & TI randomly OR AB randomly & $\begin{array}{l}\text { Expanders - Apply equivalent subjects } \\
\text { Search modes - Boolean/Phrase }\end{array}$ \\
\hline S38 & TI randomly OR AB randomly & $\begin{array}{l}\text { Expanders - Apply equivalent subjects } \\
\text { Search modes - Boolean/Phrase }\end{array}$ \\
\hline
\end{tabular}




\begin{tabular}{|c|c|c|}
\hline $\begin{array}{l}\text { Search } \\
\text { ID\# }\end{array}$ & Search Terms & Search Options \\
\hline S37 & TI randomized OR AB randomized & $\begin{array}{l}\text { Expanders - Apply equivalent subjects } \\
\text { Search modes - Boolean/Phrase }\end{array}$ \\
\hline S36 & PT "clinical trial" & $\begin{array}{l}\text { Expanders - Apply equivalent subjects } \\
\text { Search modes - Boolean/Phrase }\end{array}$ \\
\hline S35 & PT "randomized controlled trial" & $\begin{array}{l}\text { Expanders - Apply equivalent subjects } \\
\text { Search modes - Boolean/Phrase }\end{array}$ \\
\hline S34 & $\begin{array}{l}\text { S10 OR S11 OR S12 OR S13 OR S14 OR S15 OR } \\
\text { S16 OR S17 OR S18 OR S19 OR S20 OR S21 OR } \\
\text { S22 OR S23 OR S24 OR S25 OR S26 OR S27 OR } \\
\text { S28 OR S29 OR S30 OR S31 OR S32 OR S33 }\end{array}$ & $\begin{array}{l}\text { Expanders - Apply equivalent subjects } \\
\text { Search modes - Boolean/Phrase }\end{array}$ \\
\hline S33 & TI "care models" OR AB "care models" & $\begin{array}{l}\text { Expanders - Apply equivalent subjects } \\
\text { Search modes - Boolean/Phrase }\end{array}$ \\
\hline S32 & TI "care model" OR AB "care model" & $\begin{array}{l}\text { Expanders - Apply equivalent subjects } \\
\text { Search modes - Boolean/Phrase }\end{array}$ \\
\hline S31 & TI "care plan" OR AB "care plan" & $\begin{array}{l}\text { Expanders - Apply equivalent subjects } \\
\text { Search modes - Boolean/Phrase }\end{array}$ \\
\hline S30 & TI "care plans" OR AB "care plans" & $\begin{array}{l}\text { Expanders - Apply equivalent subjects } \\
\text { Search modes - Boolean/Phrase }\end{array}$ \\
\hline S29 & $\begin{array}{l}\text { TI "management plans" OR AB "management } \\
\text { plans" }\end{array}$ & $\begin{array}{l}\text { Expanders - Apply equivalent subjects } \\
\text { Search modes - Boolean/Phrase }\end{array}$ \\
\hline S28 & TI "management plan" OR AB "management plan" & $\begin{array}{l}\text { Expanders - Apply equivalent subjects } \\
\text { Search modes - Boolean/Phrase }\end{array}$ \\
\hline S27 & $\begin{array}{l}\text { TI "management processes" OR AB "management } \\
\text { processes" }\end{array}$ & $\begin{array}{l}\text { Expanders - Apply equivalent subjects } \\
\text { Search modes - Boolean/Phrase }\end{array}$ \\
\hline S26 & $\begin{array}{l}\text { TI "management process" OR AB "management } \\
\text { process" }\end{array}$ & $\begin{array}{l}\text { Expanders - Apply equivalent subjects } \\
\text { Search modes - Boolean/Phrase }\end{array}$ \\
\hline S25 & TI "training programs" OR AB "training programs" & $\begin{array}{l}\text { Expanders - Apply equivalent subjects } \\
\text { Search modes - Boolean/Phrase }\end{array}$ \\
\hline S24 & TI "training program" OR AB "training program" & $\begin{array}{l}\text { Expanders - Apply equivalent subjects } \\
\text { Search modes - Boolean/Phrase }\end{array}$ \\
\hline S23 & TI workshops OR AB workshops & $\begin{array}{l}\text { Expanders - Apply equivalent subjects } \\
\text { Search modes - Boolean/Phrase }\end{array}$ \\
\hline S22 & TI workshop OR AB workshop & $\begin{array}{l}\text { Expanders - Apply equivalent subjects } \\
\text { Search modes - Boolean/Phrase }\end{array}$ \\
\hline S21 & $\begin{array}{l}\text { TI "education materials" OR AB "education } \\
\text { materials" }\end{array}$ & $\begin{array}{l}\text { Expanders - Apply equivalent subjects } \\
\text { Search modes - Boolean/Phrase }\end{array}$ \\
\hline S20 & $\begin{array}{l}\text { TI "educational materials" OR AB "educational } \\
\text { materials" }\end{array}$ & $\begin{array}{l}\text { Expanders - Apply equivalent subjects } \\
\text { Search modes - Boolean/Phrase }\end{array}$ \\
\hline S19 & $\begin{array}{l}\text { TI "patient care guidelines" OR AB "patient care } \\
\text { guidelines" }\end{array}$ & $\begin{array}{l}\text { Expanders - Apply equivalent subjects } \\
\text { Search modes - Boolean/Phrase }\end{array}$ \\
\hline S18 & $\begin{array}{l}\text { TI "patient care guideline" OR AB "patient care } \\
\text { guideline" }\end{array}$ & $\begin{array}{l}\text { Expanders - Apply equivalent subjects } \\
\text { Search modes - Boolean/Phrase }\end{array}$ \\
\hline
\end{tabular}




\begin{tabular}{|c|c|c|}
\hline $\begin{array}{l}\text { Search } \\
\text { ID\# }\end{array}$ & Search Terms & Search Options \\
\hline S17 & $\begin{array}{l}\text { TI "patient care documents" OR AB "patient care } \\
\text { documents" }\end{array}$ & $\begin{array}{l}\text { Expanders - Apply equivalent subjects } \\
\text { Search modes - Boolean/Phrase }\end{array}$ \\
\hline S16 & $\begin{array}{l}\text { TI "patient care document" OR AB "patient care } \\
\text { document" }\end{array}$ & $\begin{array}{l}\text { Expanders - Apply equivalent subjects } \\
\text { Search modes - Boolean/Phrase }\end{array}$ \\
\hline S15 & $\begin{array}{l}\text { TI "patient care bundles" OR AB "patient care } \\
\text { bundles" }\end{array}$ & $\begin{array}{l}\text { Expanders - Apply equivalent subjects } \\
\text { Search modes - Boolean/Phrase }\end{array}$ \\
\hline S14 & $\begin{array}{l}\text { TI "patient care bundle" OR AB "patient care } \\
\text { bundle" }\end{array}$ & $\begin{array}{l}\text { Expanders - Apply equivalent subjects } \\
\text { Search modes - Boolean/Phrase }\end{array}$ \\
\hline S13 & $\begin{array}{l}\text { TI "patient care planning" OR AB "patient care } \\
\text { planning" }\end{array}$ & $\begin{array}{l}\text { Expanders - Apply equivalent subjects } \\
\text { Search modes - Boolean/Phrase }\end{array}$ \\
\hline S12 & $\begin{array}{l}\mathrm{TI} \text { "healthcare planning" OR AB "healthcare } \\
\text { planning" }\end{array}$ & $\begin{array}{l}\text { Expanders - Apply equivalent subjects } \\
\text { Search modes - Boolean/Phrase }\end{array}$ \\
\hline S11 & $\begin{array}{l}\text { TI "health care planning" OR AB "health care } \\
\text { planning" }\end{array}$ & $\begin{array}{l}\text { Expanders - Apply equivalent subjects } \\
\text { Search modes - Boolean/Phrase }\end{array}$ \\
\hline S10 & TI "health planning" OR AB "health planning" & $\begin{array}{l}\text { Expanders - Apply equivalent subjects } \\
\text { Search modes - Boolean/Phrase }\end{array}$ \\
\hline S9 & S1 OR S8 & $\begin{array}{l}\text { Expanders - Apply equivalent subjects } \\
\text { Search modes - Boolean/Phrase }\end{array}$ \\
\hline S8 & S3 AND S7 & $\begin{array}{l}\text { Expanders - Apply equivalent subjects } \\
\text { Search modes - Boolean/Phrase }\end{array}$ \\
\hline S7 & S2 OR S4 OR S5 OR S6 & $\begin{array}{l}\text { Expanders - Apply equivalent subjects } \\
\text { Search modes - Boolean/Phrase }\end{array}$ \\
\hline S6 & TI survivor OR AB survivor & $\begin{array}{l}\text { Expanders - Apply equivalent subjects } \\
\text { Search modes - Boolean/Phrase }\end{array}$ \\
\hline S5 & TI survivors OR AB survivors & $\begin{array}{l}\text { Expanders - Apply equivalent subjects } \\
\text { Search modes - Boolean/Phrase }\end{array}$ \\
\hline S4 & TI survivorship OR AB survivorship & $\begin{array}{l}\text { Expanders - Apply equivalent subjects } \\
\text { Search modes - Boolean/Phrase }\end{array}$ \\
\hline S3 & TI Cancer OR AB cancer & $\begin{array}{l}\text { Expanders - Apply equivalent subjects } \\
\text { Search modes - Boolean/Phrase }\end{array}$ \\
\hline S2 & (MM "Survivorship") & $\begin{array}{l}\text { Expanders - Apply equivalent subjects } \\
\text { Search modes - Boolean/Phrase }\end{array}$ \\
\hline S1 & (MM "Cancer Survivors") & $\begin{array}{l}\text { Expanders - Apply equivalent subjects } \\
\text { Search modes - Boolean/Phrase }\end{array}$ \\
\hline
\end{tabular}




\section{Appendix B. Data Extraction Table Content}

Table B-1. Study characteristics

\begin{tabular}{|c|c|c|c|c|c|}
\hline $\begin{array}{l}\text { Au, } \\
\text { year\{\#refID\} }\end{array}$ & $\begin{array}{l}\text { Study design } \\
\text { and } \\
\text { Purpose/Aims }\end{array}$ & $\begin{array}{l}\text { Population (include } \\
\mathrm{N} \text { and information } \\
\text { on sample that } \\
\text { might inform } \\
\text { RIGOR or } \\
\text { RELEVANCE) }\end{array}$ & $\begin{array}{l}\text { Specify the cohort used in the } \\
\text { study, where applicable (e.g., } \\
\text { CCSS, St. Jude Life, etc.) } \\
\text { Enter "NA" if not applicable }\end{array}$ & $\begin{array}{l}\text { How does this paper relate to } \\
\text { Models of Care? }\end{array}$ & $\begin{array}{l}\text { How does this paper relate to } \\
\text { Resources? }\end{array}$ \\
\hline
\end{tabular}

Table B-2. Variables and findings

\begin{tabular}{|l|l|l|l|l|}
\hline $\begin{array}{l}\text { Variables } \\
\text { evaluated }\end{array}$ & $\begin{array}{l}\text { Variables with } \\
\text { associations (specify } \\
\text { positive or negative) }\end{array}$ & $\begin{array}{l}\text { Variables without } \\
\text { associations }\end{array}$ & $\begin{array}{l}\text { Any other findings } \\
\text { as relate to IPT }\end{array}$ & $\begin{array}{l}\text { Speculations as relate to } \\
\text { context-mechanism- } \\
\text { outcome relationships (or } \\
\text { subsets thereof) }\end{array}$ \\
\hline
\end{tabular}

Table B-3. Data assessment

\begin{tabular}{|l|l|l|l|l|}
\hline $\begin{array}{l}\text { Contribution: } \\
\text { Major }\end{array}$ & $\begin{array}{l}\text { Contribution: } \\
\text { Medium }\end{array}$ & Contribution: Minor & Rigor & Relevance \\
\hline $\begin{array}{l}\text { Major: } \\
\text { Evaluates a } \\
\text { model of care } \\
\text { or resource in } \\
\text { adult survivors } \\
\text { of childhood } \\
\text { cancer }\end{array}$ & $\begin{array}{l}\text { Medium: Connects } \\
\text { some dots in IPT in } \\
\text { adult survivors of } \\
\text { childhood or adult } \\
\text { cancer and/or } \\
\text { describes a model } \\
\text { of care or resource } \\
\text { but doesn't evaluate } \\
\text { a model or resource }\end{array}$ & $\begin{array}{l}\text { Minor: Conducted in } \\
\text { non-cancer contexts but } \\
\text { mechanisms could } \\
\text { plausibly operate in the } \\
\text { context of childhood } \\
\text { cancer survivorship }\end{array}$ & $\begin{array}{l}\text { Discuss any concerns about } \\
\text { RIGOR (e.g., data credible and } \\
\text { trustworthy? Methods } \\
\text { appropriate to address research } \\
\text { goal? Research design justified? } \\
\text { Data collected to address } \\
\text { research issue?) }\end{array}$ & $\begin{array}{l}\text { Discuss any concerns about } \\
\text { RELEVANCE, such as the } \\
\text { study population (e.g., is it } \\
\text { specific to adult survivors of } \\
\text { childhood cancer), the setting } \\
\text { (e.g., do the data come from } \\
\text { outside the US), whether the } \\
\text { findings are generalizable to } \\
\text { our IPT }\end{array}$ \\
\hline
\end{tabular}




\section{Appendix C. Summary of Data Abstraction}

Table C-1. Abstraction codes*

\begin{tabular}{|l|l|}
\hline Code & Description \\
\hline Model & Models of survivorship care \\
\hline Res & Resources \\
\hline Env & Environment \\
\hline Prov F/B & Health care system and provider facilitators/barriers \\
\hline Prov Char & Provider characteristics \\
\hline Pt Needs & Patient needs \\
\hline Pt F/B & Patient facilitators/barriers \\
\hline Pt Use & Patient health service use/behaviors \\
\hline OC & Outcomes \\
\hline
\end{tabular}

* See Appendix F for details on the codes

This appendix contains a mix of text taken directly from the abstracted articles, as well as notes and paraphrasing by the review authors, identified by: "[author speculation on context-mechanism-outcome hypothesis]".

\section{Childhood Cancer Survivors}

\section{Models of Care}

\section{Prospective Studies}

Klosky 20081: Prospective, non-experimental study of 941 long-term childhood cancer survivors scheduled for attendance at the St. Jude's After Completion of Therapy long-term followup clinic between $6 / 1 / 05-5 / 31 / 06$

- Model+Pt Use: $15 \%$ of survivors classified as non-attenders

- Model+Pt Use+Pt Char+Pt Needs+Pt F/B+Env: In univariate analyses, nonattenders were more likely older, lower SES, non-white, fewer years from diagnosis, no additional cancer event, less insured, traveling by car, living shorter distance from clinic, having a scheduled social work consultation, and entering/exiting survivorship clinic

- Model+Pt Use+Pt Char+Pt F/B+Pt Needs+Env: In multivariate analysis, non-whites, uninsured, traveling by car, without secondary cancer events more likely to be nonattenders

- Model+Pt Use+Pt F/B: Work or school conflicts most commonly cited as reasons for missed appointments among non-attenders who could be contacted; others reasons listed in Figure 1

- Model+Pt Use+Pt F/B+Env: Medical care, lodging, and transportation are free at St. Jude, so these barriers probably under-represent the importance of these problems at other institutions [author speculation on context-mechanism-outcome hypothesis] 


\section{Reports From Survivors and Providers}

$\underline{\text { Blaauwbroek 2008}}^{2}$ : Survey of 133 adult survivors of childhood cancer and 115 family doctors of adult survivors of childhood cancer in the Netherlands regarding shared care by family physicians and oncologists in long term followup of adult survivors of childhood cancer

- Prov Char+Pt F/B+OC: (88\%) survivors were satisfied with the care given by the local family doctors at visit 2.14 of the 101 (14\%) survivors thought that their local family doctor's knowledge of their medical history was inadequate.

- Prov Char+Model: (82\%) participating local family doctors were satisfied with sharedcare collaboration and thought the information they had received from the LTFU clinic was adequate, $(16 \%)$ local family doctors had no opinion, and (3\%) local family doctors were dissatisfied

- Model+ Pt F/B: Before visit 1, (70\%) survivors had not received information about the possibility of late effects. At visit 3, 96\% patients received advice at the LTFU clinic and 15 patients received advice by telephone.

- Model+Pt F/B+Res: In our current study, most (85 of 121 [70\%]) of the survivors who were recalled had not received information about the possibility of late effects from treatment before their visit, and consequently, were at risk of delayed medical care if health problems were to occur. Therefore, survivors should be fully informed and family doctors should know about the possible late effects of cancer treatment and their effects on health; participation in a shared-care programm should help update family doctors' knowledge. In our study, all family doctors were given information on their patients' history, health risks, and required tests (C-O) [author speculation on context-mechanismoutcome hypothesis]

\section{Reports From Survivors}

Nathan 2008U Cross-sectional survey of 8522 participants in the Childhood Cancer Survivor Study multi-institutional cohort

- Models+Pt Use:

$\circ$ No health care $(11.2 \%)$

- General medical care (patient reported one or more medical visits, none of which was related to their previous cancer) $(57.3 \%)$

- General survivor-focused care (patient reported at least one visit related to their previous cancer, but did not report receiving advice on how to reduce risks or that screening tests for cancer-related sequelae were discussed or ordered) $(13.7 \%)$

- Risk-based, survivor-focused care (survivor-focused care that included advice about how to reduce risks or discussion or ordering of screening tests for cancerrelated sequelae) $(17.8 \%)$

- Model+Pt Char: Male survivors, those of "other" race, those with a household income $<\$ 40 \mathrm{~K} /$ year or unknown income, and high school graduates (vs. less) were more likely to report no medical visits

- Model+Pt F/B: uninsured survivors were more likely to report no medical visits

- Model+Pt Needs: Survivors who reported moderate to extreme cancer-related pain were more likely to have received care, as were those with a severe, life-threatening or disabling chronic health condition

- Model+Pt Char: Survivors who received chest radiation with or without anthracyclines were more likely to have received care 
- Model+Pt Char: Among survivors who reported some form of care, survivors who were older, male, or black were more likely to report general medical care vs. risk-based survivor-focused care

- Model+Pt F/B: Among survivors who reported some form of care, survivors who were uninsured were more likely to report general medical care vs. risk-based survivor-focused care

- Model+Pt Char: survivors who were "other race" or had more toxic therapy were more likely to report risk-based, survivor-focused care

- Model+Pt F/B: survivors who were unemployed were more likely to report risk-based, survivor-focused care

- Model+ Pt Needs: survivors who reported moderate to extreme cancer-related pain or anxiety, poor physical health, or more serious morbidity were more likely to report riskbased, survivor-focused care

- Models+Pt Char: Among survivors who received some form of survivor-focused care, being seen at a cancer center and "other" race were associated with increased odds of the care being risk-based.

- Pt Use: Among patients at increased risk for cardiomyopathy or breast cancer, $28.2 \%$ and $40.8 \%$ had undergone a recommended echocardiogram or mammogram, respectively

- Model+Pt F/B+Pt Use: Survivors who were uninsured, Canadian, lower income, or not seen at a cancer center were more likely to not report an indicated echocardiogram,

- Pt Use+Pt Needs: Survivors who had moderate to extreme anxiety were more likely to have gotten an echocardiogram

- Model+Pt Char+Pt Use: older age and care outside of a cancer center was associated with not receiving an indicated mammogram

$\underline{\text { Cassillas 2015 }}^{\text {: }}$ : Childhood Cancer Survivor Study survey of 6,176

- Pt Char+Pt Use: Male sex, an annual household income of $<\$ 20,000 /$ year, and $\leq$ high school education at baseline were independently associated with an increased risk of reporting no care at followup; race not associated

- Pt F/B+Pt Use: uninsured at risk for of reporting no care or decreased care

- Pt Needs+Pt Use: those reporting no morbidity at baseline less likely to report receiving care at followup; survivors who reported any chronic health condition at baseline were less likely to report no care at followup

- Model+Prov F/B: most were in medical system suggesting that partnerships with PCP might improve survivor-focused care [author speculation on context-mechanism-outcome hypothesis]

Christen 2016 $^{4}$ : Cross-sectional questionnaire ( $\left.\mathrm{n}=160\right)$ Swiss AYA survivors to (1) describe AYA cancer survivors' preferences for the organization and content of followup care, (2) describe their preferences for different models of followup, and (3) investigate clinical and sociodemographic characteristics associated with preferences for the different models.

- Models: Five models were evaluated: medical oncologist; hospital multidisciplinary team; pediatric oncologist; general practitioner; telephone/questionnaire

- Models+Pt Use: Of the 160 responders, $92(57.5 \%)$ reported that they still attended regular $(\mathrm{n}=84 ; 52.5 \%)$ or irregular $(\mathrm{n}=8 ; 5 \%)$ followup. Among non-attenders, $16.9 \%$ 
$(\mathrm{n}=27)$ visited their former treating doctor when they had complaints and $25.6 \%(\mathrm{n}=$ 41) never visited their former treating doctor.

- Models+Pt F/B+Pt Use: Most highly rated aspects of followup include competent staff and being taken seriously

- Models+Pt F/B+Pt Use: Medical aspects more highly rated (check for recurrence, screen late effects, educate about late effects) vs. general aspects (e.g., risk for my children, sexuality religion spirituality)

- Models+Pt F/B+Pt Use: Medical oncologist involvement most highly rated, followed by general practitioner

- Models+Pt F/B: Survivors had a clear preference for followup at the adult hospital over the GP practice and a central specialized late effects clinic. Followup at a pediatric hospital was least preferred.

- Models+Pt Use+Pt F/B: Attenders of followup rated regular appointments significantly higher, involvement of a radiotherapist significantly lower, and exchange with other survivors significantly higher vs. non-attenders.

- Models+Pt F/B: Survivors rated the followup model medical oncologist significantly higher than all other models. The followup model MDT was also rated highly and significantly higher than the two least preferred models (GP and telephone/questionnaire), but not significantly higher than followup by the pediatric oncologist. Regarding hospital-based followup by a MDT, survivors agreed that they could contact the specialists they needed or would be referred to the right specialist. Survivors believed that followup by a MDT could be less personal. They saw less of a problem that they would not know who was responsible for them.

- Models+Pt Char+Pt F/B: Females rated the followup model MDT significantly higher than males. Survivors not attending followup rated the model telephone/questionnaire higher than survivors still attending followup. Attenders of followup reported lower satisfaction with the GP model than nonattenders.

- Models + Pt F/B: We found that it is important for AYA cancer survivors to be treated by competent staff and to be taken seriously. This might be especially important for this group who is still at a young age. However, many of them have considerable knowledge about their disease and treatment and might distrust medical staff with little specialization in oncology. [author speculation on context-mechanism-outcome hypothesis]

- Models+Pt F/B: Although our survey was sent a considerable time after diagnosis, the survivors' biggest concern still was cancer relapse, followed by concerns about occurrence of late effects. These concerns might explain why survivors rated the medical oncologist as the most important specialist to be involved in followup. Survivors seem to believe that a medical oncologist would be best suited to detect a new cancer or new late effects. [author speculation on context-mechanism-outcome hypothesis]

- Models+Pt Needs: A reason why non-attenders of followup rated the GP and telephone/questionnaire model higher than attenders might be that non-attenders are healthier or suffer from fewer or less severe late effects than attenders. In our sample, non-attenders were less likely to report late effects than attenders of followup. [author speculation on context-mechanism-outcome hypothesis]

- Models+Pt Char: Women rated the model MDT higher than men, possibly because they expect that the MDT model will include supportive care to a higher level than the other models of followup. Previous research has shown that women expect psychological 
support at followup [18], have more late effects [2], and rate supportive care higher than men [17]. This raises the question whether followup care should be organized differently for male and female survivors. [author speculation on context-mechanism-outcome hypothesis]

- Models+Pt Needs+Pt F/B: Because of financial constraints, it is difficult, and from a clinical point of view also not necessary, to follow all survivors by a medical oncologist or a MDT. Therefore, alternative models have to be considered. GP-led followup might be acceptable to survivors who no longer want to attend hospital-based followup. Close collaboration with specialists and the use of survivorship passports [19] could guarantee high-quality followup for these survivors. [author speculation on context-mechanismoutcome hypothesis]

$\underline{\text { Svedberg 2016 }}^{5}$ : Mixed methods study to obtain the perspectives of 213 Swedish young adult survivors of childhood acute lymphoblastic leukemia on the healthcare services they received after treatment

- Models+Pt Use: $14 \%$ had maintained contact with pediatric care and 30\% participated in regular check-ups.

- Pt Use+Prov Pract: The respondents who had no regular contact with healthcare services reported to a higher degree that they had not received knowledge, treatment strategies and guidance for coping with physical changes. Furthermore, those who were generally satisfied with the level of contact reported that they received knowledge and treatment strategies regarding physical changes to a higher degree than those who were generally dissatisfied with the level of contact. However, even if the respondents were satisfied with the level of contact the majority reported that they had not received sufficient knowledge and treatment strategies about physical changes.

- Pt Use+Prov Pract: There was a significant difference in experience of receiving knowledge and treatment strategies regarding psychological changes between the group that had regular clinical check-ups and the group that did not have any contacts with care services, as well as between the group who were satisfied with the level of contact and those who were not. However, there were no differences between the groups regarding guidance with psychological changes. Even if the respondents, who were satisfied with the level of contact, reported that they received a higher level of knowledge and treatment strategies about psychological changes for continuing life after the illness than those who were dissatisfied with the level of contact, as many as two thirds of the former reported a low level of support.

- Pt Use+Prov Pract: There were significant differences between respondents who attended regular clinical check-ups and those who had no contact with healthcare services in terms of knowledge and treatment strategies related to social changes, but not to guidance in coping with social changes.

Cassillas 2011 ${ }^{6}$ : Survey of 376 young adult cancer survivors (LIVESTRONG)

- Model+Pt Use+Env: 73\% report they currently visit survivorship or oncology clinic, with $45 \%$ reporting having to travel greater than 30 miles

- Res+Pt F/B: $33 \%$ pts no med records; $48 \%$ no treatment summary; $55 \%$ no SCP; $19 \%$ none of any of these three. 
- Model+Prov Char+Pt F/B: Most participants indicated that their oncologist played the dominant role in tests and treatment decisions (70\%), providing cancer care in the next 6 months $(69 \%)$, and was most likely to know about or treat symptoms $(56 \%)$; PCPs scored much lower in these three areas of survivorship care $(4 \%, 10 \%$, and $18 \%$, respectively) as did the "shared-care model" (10\%, 5\%, and $6 \%$, respectively); no association was found between interval since cancer diagnosis and the predominant care model

- Pt F/B: 41\%(155/376) were classified as low confidence in managing their survivorship care using the five-item composite index.

- Model+Res+Pt Char: Education (high in this sample) and receipt of care at a cancer clinic seemed to NOT positively impact the receipt of survivorship resources $(\mathrm{M} / \mathrm{C})$ [author speculation on context-mechanism-outcome hypothesis]

- Res+Prov F/B+Prov Pract: The factors that may account for the lack of receipt of a survivorship care plan include outpatient infrastructure barriers and a focus on screening for cancer recurrence in the outpatient oncology setting rather than on health promotion and disease prevention for survivors (M); Other barriers to SCPs: outpatient setting (no incentive) $(\mathrm{M} / \mathrm{C})$ [author speculation on context-mechanism-outcome hypothesis]

- $\operatorname{Res}+\mathbf{P t}$ F/B: Survivors may not remember receipt $(\mathrm{C} / \mathrm{M})$

- Pt F/B+OC: Deficits in any one area, for example having a survivor who is neither wellinformed nor participatory in their survivorship care planning, can ultimately impact on their long-term health outcomes $(\mathrm{C} / \mathrm{O})$ [author speculation on context-mechanismoutcome hypothesis]

Absolom 2006 $^{7}$ : Cross sectional study (with 2 timepoints) of 198 survivors treated for cancer before age 16, currently aged 16-40 years, being treated at 2 hospitals in northern England to compare various measures between survivors of childhood cancers being seen in adult vs. pediatric clinic for follow up.

- Models+Pt Use: Survivors attending the paediatric clinic discussed a greater number of topics than those at the adult clinic (Table 2). Concerns about past treatment $(29.7 \% \mathrm{vs}$ $50.7 \%, \mathrm{v} 2=6.16, \mathrm{P}<0.01)$, health behaviours $(37.3 \%$ vs $95.9 \%, \mathrm{v} 2=55.63, \mathrm{P}<0.001)$ and insurance $(7.4 \%$ vs $49.3 \%, \mathrm{v} 2=29.56, \mathrm{P}<0.001)$ were discussed more frequently in the paediatric clinic.

- Models+OC: Survivors attending the paediatric clinic reported greater satisfaction than those attending the adult clinic. There was no significant direct effect of clinic type on satisfaction, but clinic type had an effect on number of topics $(\mathrm{b}=0.44, \mathrm{P}<0.01$; survivors reported discussing more topics at the paediatric clinic), which in turn had an effect on satisfaction $(b=0.31, \mathrm{P}<0.01)$.

- Models+Pt Needs: Survivors at the two clinics did not differ in number of symptoms or late-effects, vulnerability or perceived purpose of follow up.

- Models+Pt F/B: Perceptions of time waiting in clinic or time with staff did not differ between the two clinics. Clinic type was not associated with perceptions of time waiting, length of consultation, or reasons for followup.

- Models+Pt F/B: Our findings suggest that survivors themselves may be less affected by differences in location and organisation of clinics than assumed by medical staff. Regardless of the service offered, practical issues about timing and opportunities to discuss more health issues were found to be most critical to satisfaction. 
$\underline{\text { Landier 2015 }}^{8}$ : 5 health knowledge questionnaires completed, 1 before initial survivorship clinic visit and others after. Assessing knowledge of therapy and therapy-related health risk in 369 adult survivors of childhood cancers from single institution; off cancer therapy $>2 \mathrm{yrs}$

- Model+Res: survivorship clinic, plus tailored educational materials about therapy-related late effects to be used at each LTFU visit with NP or MD

- Model+Res+Pt F/B: Descriptive results: largest gain was awareness of neurocognitive impairment, smallest gain was for renal dysfunction. $60 \%$ of survivors remained unaware of most of their risks ( $>75 \%)$ even after repeated, tailored interventions.

- Res+Prov Char+Pt F/B: Proportion of personal health risks identified increase from $38.6 \%$ at baseline to $66.4 \%$ at time 3 . No significant gain after that. Patients with lowest awareness at baseline had the greatest gains, and gains didn't differ by clinician type (MD vs NP)

- Pt Char+Pt F/B: baseline low levels of knowledge associated with education, time since diagnosis, leukemia (vs. other diagnoses), nonwhite race and risk of fewer complications.

Signorelli 2019': survey and optional interviews to assess barriers to accessing and preferences for survivorship care in $\mathrm{N}=633$ : 187 parents of $<16$ years; 251 AYA; 195 (mean age 32.5); interviews with 151 survivors of childhood cancer in Australia

- Env+Pt Char+Pt F/B: older survivors in rural/remote areas endorsed more barriers than survivors in metropolitan areas (mean: 5.2 vs 4.6; $\mathrm{p}=0.006$ ). as did those longer from time of diagnosis. Hospital-based survivorship care - involving an oncologist or other clinic doctor, cancer survivorship nurse or team - was most commonly endorsed as the 'first choice' across all age groups (97\% parents, 88\% AYAs, 86\% older survivors)

- Model: lower confidence in PCPs; other specialists requested: psychologists, fertility specialists; preferred setting: outpatient hospital; flexible times

- Pt F/B: two-thirds (includes parents) noted logistical barriers that are modifiable

$\underline{\text { Signorelli 2020 }}^{\mathbf{1 0}}{ }^{\text {: }}$ Survey pre-post pilot study of nurse-led distance-delivered intervention in 27 childhood cancer survivors, all classified as high risk

- Model: "Re-engage" designed to promote cancer-focused and risk-appropriate care (details of intervention in Figure 1)

- Model+Env+Pt F/B: asked respondents why did not engage in survivorship care; most common reason was distance to clinic and low perceived need for care; self-efficacy increased post-intervention

- Model+Pt F/B: speculate program could empower pts to manage care and change behaviour[author speculation on context-mechanism-outcome hypothesis]

$\underline{\text { Szalda 2016 }}^{11}$ : online survey of 99 young adult survivors ( 80 with complete data) who were transferred to adult-focused followup care in past 1-5 years about engagement and experience after transfer to adult care

- Model: specialized practice (adult survivor or oncology), PCP, shared (both special and PCP)

- Model+Pt F/B: There was no significant difference in engagement (perceptions about cancer-related followup) by model; no difference in perceived quality of care received by model; overall satisfaction was generally high and did not differ by model/provider type 


\section{Model+Pt Use+Pt Char: That all patients formally transferred to adult care}

did not report receiving recommended care in the prior year indicates that patient-specific predictors of transition readiness and followup care should be explored. [author speculation on context-mechanism-outcome hypothesis]Reports From Providers

$\underline{\text { Suh 2014 }}^{12}$ : cross sectional survey of 1110 internists in AMA physician Masterfile regarding preferences/knowledge of care of adult survivors of childhood cancer

- Prov Char+Prov F/B: men had higher comfort levels seeing CCS pts than women

- Prov Pract+Prov F/B: internists with larger pt volume and who saw at least 1 CCS in last 5 years had higher comfort levels in seeing CCS pts

- Prov Pract+Prov F/B: internists who saw at least 1 CCS in last 5 years associated with promoting annual breast cancer surveillance (both mammo+MRI and mammo only)

- Prov Char+Res: 51\% reported having cared for at least 1 adult CCS in the past 5 years, $72 \%$ never received treatment summary

- Model+Prov Char: 5.5\% preferred to care for CCS independently, $84 \%$ preferred working in collaboration with cancer center based physician or LTFU clinic, $10.5 \%$ said they would refer pt to cancer center based physician, LTFU clinic, or another PCP

- Prov Char+Prov F/B: on average, internists somewhat uncomfortable caring for CCSs: $37 \%, 27 \%, 25 \%$ somewhat comfortable/comfortable caring for Hodgkin, ALL, osteosarcoma survivors

- Prov Char+Res: internists generally unfamiliar with available surveillance guidelines for CCS (12\% somewhat familiar)

- Prov Char+Prov Pract: clinical vignette assessed surveillance guidelines for breast cancer, cardiac, thyroid function -- 9\% were in concordance with guidelines for breast cancer surveillance in women with chest radiation $\mathrm{hx}$ (mammo+MRI), additional 18\% recognized need for mammo (but not MRI), $15 \%$ identified biennial echo for cardiac monitoring, 76\% assessed thyroid function appropriately; $5 \%$ were concordant with all 3 surveillance questions

- Prov Char+Res: most useful tools for caring for adult CCSs: LTFU guidelines ( $86 \%$ "very useful") and pt specific standardized letters from specialists with f/u recs for PCPs sent directly to PCP ( $80 \%$ "very useful")

$\underline{\text { Sima 2014 }}^{13}$ : cross sectional survey of 351 US PCPs (general internal medicine or family practice) to identify facilitators and barriers to PCPs to provide optimal care for adult survivors of childhood cancer

- Prov Char+Res: more years since graduating from med school associated with having received an SCP (other prov char NS)

- Prov Char+Res: more years since graduating from med school less likely to find SCP useful

- Prov Char+Res: women more likely to strongly agree that TS is useful, agree that SCP is useful; regional differences geographically that $\mathrm{TS} / \mathrm{SCP}$ is helpful

- Model+Prov Char: 40\% said they never cared for CCS, 37\% were neutral or disagreed that problems of CCS were important to their practice 
- Res+Prov Char: 40\% aware of CCS practice guidelines, felt their training was adequate to recognize late effects (27-38\% for chemo, surgery, radiation -- higher for surgery and radiation compared to chemo)

- Prov Char+Prov F/B: Barriers: adequate time to discuss late effects (42\%), survivors do not see late effects as health risk (33\%), insurance coverage for screening of late effects is a problem for adult CCS (39\%), difficult to get cancer history from pts $(65 \%)$ and from oncologist (51\%),

- Prov Char+Res: $84-86 \%$ reported never receiving a cancer treatment summary or survivorship care plan, 93\% never used COG late effects guidelines; $>90 \%$ PCPs thought it would be useful to receive TS or SCP, $86 \%$ agreed they would follow COG late effects guidelines; rank order importance in source of info: oncologist $45 \%$, internet $33 \%$, books $10 \%$, journal articles $7 \%$, primary care colleagues $3 \%$, other specialists $2.5 \%$; most useful format for medical screening prompt: letter (41\%), computer prompt or flowsheet in chart $(35 \%)$, phone call to office $(11 \%)$, emailer reminder $(7 \%)$, website with pt info $(6 \%)$

Guilcher 2009 $^{14}$ : Cross-sectional survey of 16 pediatric hematology/oncology programs regarding long-term followup care programs for childhood cancer survivors in Canada

- Model: 13/15 centers (87\%) had a formal long-term followup program, defined as a dedicated program for patients greater than 2 years beyond active treatment

- Model+Pt Use: 8/15 of long-term followup centers (53\%) had a formal program for adult survivors of childhood cancer. only 4 of these 10 programs felt that $50 \%$ or greater of their adult survivors were followed in their formal program.

- Model+Pt Needs+Pt Char: "Most important determinants to whether a childhood cancer survivor is followed in a formal adult late effects program were: existing late effects at the time of transfer, potential adult onset of late effects, and disease status (short time from completion of therapy)."

\section{Other Study Designs}

$\underline{\text { Marr 2017 }}^{15}$ : Retrospective population-based study using administrative data to determine if attendance at a specialized clinic for adult survivors of childhood cancer is associated with better rates of adherence to the Children's Oncology Group (COG) Long-Term Follow-Up (LTFU) guidelines for cardiomyopathy screening. $\mathrm{N}=1811$ 5-year survivors diagnosed between 1986 and 2005 at risk of therapy-related late cardiomyopathy (Ontario, Canada)

- Model: Specialized survivor clinic: The Ontario Pediatric Oncology AfterCare Program (a provincial network of 7 LTFU clinics for adult survivors of childhood cancer located at pediatric or adult cancer centers within 5 major cities in the province)

- Model+Pt Use: More frequent use of survivor clinics is highly associated with rates of adherence to cardiac screening guidelines. The relative rate of adherence increased 10.6fold for patients with highest risk of secondary cardiomyopathy (screening recommended every year), 3.3-fold for patients needing screening every 2-years, and 2.3-fold for patients needing screening every 5-years (compared to no attendance)

- Model+Pt Use: Our results show that dependence on survivor clinics alone is insufficient to ensure complete adherence to guidelines: only $55 \%$ of at-risk survivors underwent even one echocardiogram. This study was unable to identify how the rates of adherence 
differ for patients managed with survivor care combined with primary care compared to those in primary care alone and this is an area that would benefit from further study.

- Pt Char+Pt Use: Female sex was independently associated with increased adherence

- Pt Char+Env+Pt Use: Unlike prior studies of this cohort, higher treatment intensity, higher SES, and decreased distance from the clinic were not independently associated with increased adherence.

$\underline{\text { Nathan 2016 }}^{16}$ : Retrospective study linking data from a population-based pediatric cancer registry with health administrative databases to determine attendance at 5 specialized survivor clinics on Ontario between 1999 and 2012. N=912 children and adolescents cancer survivors

- Model+Pt Use: After a median of 7.8 years of followup, $43.3 \%$ had attended at least one adult survivor clinic visit

- Model+Pt Char+Pt Use: Significantly increased rates of attendance were associated with female gender, higher treatment intensity, radiation, higher alkylating agent exposure, higher SES, and an annual exam by a PCP.

- Model+Env: Distance (living >50 kim away) were less likely to attend than those living within $10 \mathrm{~km}$.

- Model+Pt F/B+Prov F/B: Despite free access to survivor clinics, the majority of survivors do not attend. Lack of insurance should not be a barrier; other barriers might include co-pays for medical visits / surveillance tests (for low-income patients) and indirect costs (e.g., time of work, travel). (From Discussion): The barriers to providing appropriate care to adult survivors of childhood cancer appear to be three-fold: (1) there is a paucity of specialized survivor clinics, (2) even when such clinics exist, many survivors do not attend, (3) most PCPs lack the comfort and knowledge to provide care to survivors, particularly in the absence of cancer center support. Given the inconsistency with which survivors attend these clinics and the general discomfort among PCPs, empowerment of the survivor to seek appropriate care (regardless of care location or provider) is critical. However, survivor knowledge about their diagnosis, treatment, and consequent risk for late effects is often inadequate, creating a significant barrier to seeking appropriate care. [author speculation on context-mechanism-outcome hypothesis]

\section{Combined Model and Resource (e.g., SCP)}

\section{Prospective Studies}

Oeffinger 2011 $^{17}$ : Prospective one-arm study of effect of a SCP and information provision intervention in 62 HL survivors aged 27-55 from the Childhood Cancer Survivor Study at increased risk for breast cancer or cardiomyopathy who had not had mammogram or ECG in 2 years

- Model+Res: mailed one-page SCP to patient; PCP also contacted and provided patientspecific information; virtual information center (web-based resource designed specifically for HL survivors and physicians) available to patient and PCP

- Res+Pt Use: $78 \%$ of survivors remembered receiving SCP in the mail and nearly all had read and understood it; 39\% wanted more risk information; nearly half of those who had seen a physician since SCP receipt had shared it; most who hadn't seen a physician planned to share the SCP when they did

- Res+Pt Use: $29 \%$ of survivors visited the website. More women than men. 
- $\operatorname{Res}+\operatorname{Prov} \mathbf{F} / \mathbf{B}$ : None of physicians who agreed to participate in the study visited the website or contacted the study team.

- Res+Pt Char+OC: Male participants reported greater fatigue after study than before $(\mathrm{p}=0.02)$

- Model+Res+OC: patient mood and anxiety didn't change with intervention; participants reacted favorably to SCP

- Model+Res+Pt Use: After study 41\% of survivors reported mammogram, 20 (CS: \%?) reported ECG (more women than men). Additional 1/3 in both groups reported plans for screening. increased levels are suggestive of the intended effect

- Res+Prov Pract: PCPs seemed too busy to be involved in the study or to utilize resources, but they ordered tests when the patient presented them with information on risks.

$\underline{\text { KadanLottic 2018 }}^{\mathbf{1 8}}$ : Randomized trial evaluating SCP provision to and implementation by PCP

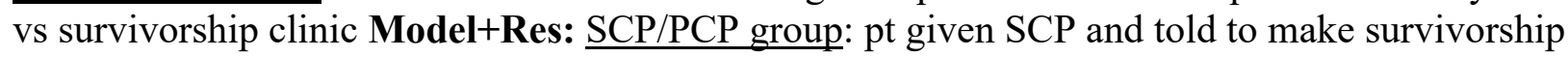
care focused visit to PCP in the next 12 months, PCPs given general educational survivorship materials (but not SCP) and info to contact study team $v s$ survivorship clinic (SC) group: Yale pediatric survivorship clinic visit in next 12 months, which included H\&P, guideline recommended surveillance testing, and education on health behaviors, SCP given at the end of the visit

- Model+Res+Pt Use: Markedly higher patient adherence to getting testing (esp bone density test, echo, blood/urine tests esp gonadotropins) in SC group vs SCP/PCP

- Model+Res+Prov Pract: Markedly higher provider adherence to ordering testing (esp bone density test, echo, blood/urine tests esp gonadotropins) in SC group vs SCP/PCP

- Model+Res+Prov Pract: markedly higher number of late complications identified in SC group (11 patients, 21 complications) vs SCP/PCP (1 patient, 1 complication)

- Model+Res+Prov Pract: many late complications are not detectable by self-report or physical examination and required specialized testing...our data suggest that lack of identification of late complications was due to lack of ordering recommended surveillance tests. [author speculation on context-mechanism-outcome hypothesis]

\section{Reports From Survivors and Providers}

Costello 2017 ${ }^{19}$ : Cross-sectional observational pilot study to evaluate shared care in the form of a telemedicine transition visit with a PCP/CCS joined by pediatric survivorship clinic team member; 19 transition visits for childhood cancer survivors age $18+$ who were $10+$ years posttreatment with a PCP also willing to participate

- Model+Res: Evaluates a telemedicine visit conducted with primary care provider/CCS dyads joined by a pediatric survivorship clinic team member who reviewed an individualized treatment summary and care plan. Transition visit included survivorship care plan and followup guidelines based on the Children's Oncology Group recommendations.

- Models+Res+Prov F/B: Underlying discomfort with using telemedicine equipment in PCP offices (resulting in low response rate; 24 of 46 eligible dyads agreed to participate); only $13 / 19$ visits could use the telemedicine equipment due to technical difficulties with the rest using audio only 
- Models+Res+Pt F/B+Prov F/B+OC: Postquestionnaires were overall positive, confirming increased knowledge, comfort and abilities, and patient satisfaction in survivorship care. Negative comments were primarily related to equipment difficulties.

\section{Reports From Survivors}

$\underline{\text { Yan 2020 }}^{\mathbf{2 0}}$ : Survey to assess current adherence to COG and ACS cancer and cardiac surveillance guidelines, and to explore predictors of adherence in 11337 survivors of childhood cancer at high risk for second malignant neoplasms or cardiac dysfunction and 2146 siblings

- Res+Pt Char: Compared with survivors treated between 1970 and 1979, survivors treated between 1980 and 1989 and between 1990 and 1999 were more likely to have a SCP

- Pt Char+Pt Use: Among high-risk, adherence to breast, colorectal, skin, and cardiac surveillance was $12.64 \%$ (CI, $10.0 \%$ to $15.3 \%$ ), $37.0 \%$ (CI, 34.1\% to $39.9 \%$ ), $22.3 \%$ (CI, $21.2 \%$ to $23.4 \%$ ), and $41.4 \%$ (CI, 40.1 to 42.7 ), respectively;

- Pt Char+Pt Use: Among average risk, adherence to ACS breast, cervical, and colorectal screening was $57.1 \%$ (CI, $53.2 \%$ to $61.0 \%$ ), $83.6 \%$ (CI, $82.7 \%$ to $84.5 \%$ ), and $68.5 \%$ (CI, $64.7 \%$ to $72.2 \%$ ), respectively

- Res+Pt Use: In multivariable analyses, among high-risk, survivor-reported SCP possession was associated with increased adherence to breast (22.3\% v 8.1\%; PR, 2.52; CI, 1.59 to 4.01$)$, skin (34.8\% v 23.0\%; PR, 1.16; CI, 1.01 to 1.33$)$, and cardiac $(67.0 \% \mathrm{v}$ $33.1 \%$; PR, 1.73; CI, 1.55 to 1.92 ) surveillance

- Pt Use+Pt Use: Among high-risk, survivors associated with increased adherence to skin surveillance $(39.6 \%$ v $23.2 \%$; PR, 1.24 ; CI, 1.08 to 1.43$)$. Having a check-up related to past cancer in the past 2 years (regardless of location or provider) increased breast (PR, 7.94; CI, 1.99 to 31.74), skin (PR, 1.50; CI, 1.28 to 1.76), and cardiac (PR, 1.58; CI, 1.39 to 1.80) surveillance. Visiting a doctor more than 5 times in the past 2 years increased skin (PR, 1.47; CI, 1.28 to 1.69) and cardiac (PR, 1.06; CI, 0.99 to 1.14) surveillance

- Model+Pt Use: Visiting a special cancer survivorship clinic in the past 2 years increased cardiac surveillance (PR, 1.16; CI, 1.04 to 1.30), but visiting a cancer specialist did not increase adherence to any of the guidelines

- Res+Prov Char+Pt Use: Among average risk, PCP SCP possession was associated with increased adherence to breast (PR, 1.28; CI, 1.13 to 1.45 ) and colorectal (PR, 1.12; CI, 1.04 to 1.21$)$

- Pt Char+Pt Use: fewer than half of high-risk survivors receive the recommended surveillance (C) [author speculation on context-mechanism-outcome hypothesis]

- Res: Despite the IOM's recommendation and the Commission on Cancer's mandate, few survivors and PCPs have a SCP; survivors treated after 1990 were more likely to have a SCP, suggesting that dissemination is increasing, potentially because of these recommendations (C-M-O) [author speculation on context-mechanism-outcome hypothesis]

- Res: Despite the current suboptimal adherence to COG guidelines, adherence improved between 2003 and 2016. This was most evident for colorectal (from 14.3\% to 41.0\%) and cardiac surveillance (from $22.4 \%$ to $38.5 \%$ ). Reasons for these increases are likely multifactorial. In 2012 , only $12 \%$ of general internists 25 and $9 \%$ of family doctors 26 felt at least "somewhat familiar" with care guidelines for childhood cancer survivors. Physician awareness of COG guidelines may be increasing as the time from initial 
publication grows. (C-M) [author speculation on context-mechanism-outcome hypothesis]

$\underline{\text { Berg 2016 }}^{\text {21. }}$ : Mixed methods study in survivors of childhood cancer aged 18-34 recruited from a university affiliated children's hospital and an NCI designated cancer center in Southeastern USA, including surveys $(\mathrm{n}=106)$ and phone-based semi-structured interviews with a subset of 26

- Models+Pt Use: $46 \%$ of survey participants had attended a survivorship clinic; $68.9 \%$ had a PCP; $17 \%$ reported no interaction with healthcare in the past 2 years, with an additional $14 \%$ reporting only seeing a provider once; 1.66 average years since had clinic visit with oncologist; .96 years since had clinic visit with survivorship clinic (among those who had a visit; $54 \%$ had never had a visit)

- Res: $42.2 \%$ reported receiving a treatment summary

- Prov Pract: $63.2 \%$ reported that, during their last clinic visit, a provider discussed any symptoms related to their cancer, with less than half reporting that they promoted adherence to care recommendations $(44.3 \%)$, interest in reproduction $(42.5 \%)$, social support (39.6\%), and mental health issues (49.1\%)

- Pt Use+Pt Char: In bivariate analyses, factors associated with less than annual healthcare provider visits included being older, being male, not being employed, lack of insurance, having chemotherapy, moving

- Pt Use+Pt Char: In multivariate analyses, factors associated with less than annual healthcare provider visits included being older, being male, lack of insurance, having chemotherapy

- Res: In terms of resources, several suggestions for using technology to facilitate communication with providers, the potential utility of blogging or message boards to promote education and support, and internet forums/social networks of young adult cancer survivors provide support

\section{Reports From Providers}

Blaauwbroek 2007 ${ }^{22}: 10$-item post-course questionnaire to assess the willingness of GPs ( $\mathrm{n}=$ $233 / 358,65 \%$ response rate), who had followed a postgraduate course on late effects of cancer treatment in the Netherlands, to participate in a shared care model for followup of adult childhood cancer survivors as well as what their requirements would be in case of participation

- Res+Model: $97 \%$ were willing to participate in a shared care model for followup; 64\% felt that it was their responsibility to be in charge of childhood cancer survivors.

- Res+Model+Prov F/B: Main requirements for participation were availability of guidelines (64\%), sufficient information about the patient's medical history (37\%), and short communication lines (45\%); main barriers were workload $(16 \%)$, lack of knowledge (15\%), and lack of communication (13\%).

- Prov Char+Model: GPs are divided on the issue, some viewing shared care as improving job satisfaction and others as another example of hospitals offloading work onto an already overloaded primary care sector. [author speculation on contextmechanism-outcome hypothesis]

Iyer $\mathbf{2 0 1 7}^{\mathbf{2 3}}$ : mixed methods study of PCP perspectives $(\mathrm{n}=134)$ on SCPs and survivorship care for childhood cancer survivors who had attended Yales' HEROS Survivorship Clinic

- PCP CHARACTERISTICS: 
○ PCPs in practice $\sim 28$ years $+/-10$ (6-60), $54 \%$ peds, $23 \%$ internists, $20 \%$ family practice

- PCPs treated 1-4 pts (ave 1, 84\% only 1 ) childhood cancer survivors in the last 5 years

○ PCPs: $55 \%$ had at least some education on survivorship care/late effects, $34 \%$ had seen $>5$ late effects, $45 \%$ had seen at least 1 late effect grade 3 or higher

- SURVIVOR CHARACTERISTICS

○ 162 survivors across 134 PCPs, age $7.5+/ 5.8$ years $(0-20)$ at diagnosis, mean age $18.2+/-8.3(3-49)$ at first survivorship visit

○ survivors' cancers: 40\% leukemia, 18\% lymphoma, 16\% sarcoma, 10\% CNS tumor; treatment: $91 \%$ chemo, $51 \%$ radiation, $48 \%$ surgery, $13 \%$ BMT

○ average 4 late effects (0-16), $18 \%$ had none, of 133 with a late effect, $52 \%$ had grade 3 or higher

- Baseline survey results of PCPs

○ $46 \%$ PCP most preferred specialty survivorship clinic as model of care, then shared $(26 \%)$, oncologist alone (21\%), PCP alone $(8 \%)$

- $31 \%$ very comfortable providing recommended surveillance on SCP, $19 \%$ very comfortable using SCP to provide recommended surveillance of neurocognitive late effects, $3 \%$ very comfortable providing all aspects of survivorship care

$\circ$ All PCPs were sent pts SCP, though only 51\% recalled receiving it -- of those recalling SCP receipt, $75 \%$ of PCPs reported often or always reviewing the plan, $42 \%$ discussing SCP with patient

○ $48 \%$ found treatment summary very helpful, $56 \%$ found $\mathrm{f} / \mathrm{u}$ surveillance recommendations helpful

- $91 \%$ had at least one moderate or large barrier to providing survivorship care -$74 \%$ lack of knowledge/training, 69\% incomplete/unclear SCPs, 58\% unclear designation of responsibility; $48 \%$ lack of communication with pediatric pncologists $45 \%$ lack of access to specialists who provide specific aspects of survivorship care, $41 \%$ lack of patient willingness to get survivorship care with PCP, 29\% lack of reimbursement

- Prov Char+Prov F/B: no association between provider characteristics and provider preferences for survivorship care

- Pt Char+Prov F/B: no association between patient characteristics and provider preferences for survivorship care

- Prov Char+Prov F/B: no association between provider characteristics and cited barriers to survivorship care

- Pt Char+Prov F/B: no association between patient characteristics and cited barriers to survivorship care

- Res+Models+Prov F/B: Our data suggests that before collaborative models with PCPs can be effectively expanded, greater emphasis must be focused on how to best address knowledge gaps related to the SCP, its use, and survivorship care more generally. Passive distribution of the SCP does not seem to be adequate. [author speculation on contextmechanism-outcome hypothesis]

Gisela 2017 ${ }^{\mathbf{2 4}}$ : Cross-sectional survey of providers (27 medical oncologists, 13 pediatric oncologists, 122 general practitioners, and 21 pediatricians) in Switzerland regarding roles and 
problems in followup care for childhood cancer survivors regarding (1) involvement in followup care; (2) content of followup care provided; (3) problems encountered; and (4) additional resources needed.

- Models: $81 \%$ of medical oncologists, $85 \%$ of pediatric oncologists, $39 \%$ of GPs and $81 \%$ of pediatricians were involved in followup.

- Prov Char+Prov Pract: Compared with generalists, more oncologists examined survivors for relapse, second tumors and late somatic effects, and informed them about their former disease, former treatment and possible late effects. Differences were less pronounced for the examination of psychological late effects; counselling on healthy behavior did not differ between oncologists and generalists.

- Prov Char+Prov F/B: Pediatric oncologists reported more problems than medical oncologists: many pediatric oncologists reported problems with transition of survivors to adult care (91\%), and because of financial resources (73\%) and time restraints $(73 \%)$. Medical oncologists reported most problems during transition (23\%). Not being aware of a survivor was the most common reason for GPs and pediatricians not participating in followup (74\%). GPs (94\%) and pediatricians (100\%) additionally desired more support from oncologists.

- Prov Char+Res: All groups reported a need for standardized protocols (85-91\%) and specialized training $(55-73 \%)$.

- Prov Char+Prov F/B+Res+Models: Generalists need to be educated and provided with guidelines sufficiently to feel comfortable in examining and discussing cancer-related issues. This was tried in a pilot project and proved effective in terms of feasibility and interest of primary care physicians [43]. One specialized primary care physician for a certain catchment area might be able to provide adequate followup care for all survivors in the area and involve other specialists if needed. [author speculation on contextmechanism-outcome hypothesis]

- Res: Each survivor should receive a personal survivorship care plan, a so called "survivorship passport". [author speculation on context-mechanism-outcome hypothesis]

\section{Survivorship Care Plans}

\section{Randomized Controlled Trials or Related}

Hudson 2014 ${ }^{25}$ : RCT to determine whether addition of tailored counseling by advanced practice nurses to mailed SCP increases proportion of at-risk Childhood Cancer Survivor Study

participants ( $\mathrm{n}=472 ; 411$ completed) who complete cardiomyopathy screening

- Res: Addition of 2 telephone counseling sessions 1 and 3 weeks after SCP with advanced practice nurse tailored to address barriers to completion of cardiomyopathy screening

- Res+Pt Use: 107 (52.2\%) of counseling arm vs 46 (22.3\%) completed screening at 1 year $(\mathrm{p}<0.001)$. No factors were identified that modified the effect of the intervention.

- Pt F/B: Of those who didn't get the screening, reasons cited were lack of time, not perceived as important, concerns about insurance coverage, couldn't afford/no insurance, not recommended by physician, forgot. Lack of physician recommendation more likely in standard care group ( $19.9 \%$ vs $8.2 \%, \mathrm{P}=0.02)$

- Model+Pt Char: Screening detected cardiomyopathy in $10 \%$ and other abnormalities consistent with evolving cardiac dysfunction. Need for regular communication between 
pediatric oncology and PCPs to facilitate understanding the unique and emerging health risks with treatment for childhood cancer.

- Res+Pt F/B: Concerns about insurance coverage for screening was a barrier, even more so in the intervention group where resources related to this were provided

Murphy 2021 26: Randomized controlled trial (Intervention, $\mathrm{n}=46$; Control, $\mathrm{n}=64$ ) evaluating Survivorship Healthcare Passport in childhood cancer survivors

- Res: This passport is based on the SCP that each patient also receives and is a durable, portable, wallet sized plastic card that provides a summary of the patient's diagnosis and treatment history along with a succinct assessment of late effects risks associated with their treatment. The passport includes recommendations for followup care based on the Children's Oncology Group (COG) Long-Term Follow-Up Guidelines of Childhood, Adolescent and Young Adult Cancers along with our institutional practice guidelines.

- Res+Pt F/B: Improved knowledge of those given passport card for diagnosis, treatment, risks, and recommended followup. The majority of those with a passport (55.8\%) showed a greater than 20-point knowledge gain in their knowledge score compared with $26.2 \%$ of those without a passport between the baseline questionnaire to the questionnaire 1 month after the clinic visit.

- authors speculate on role in relationship with PCP; increase compliance. [author speculation on context-mechanism-outcome hypothesis]

\section{Prospective Cohort Studies}

$\underline{\text { Pannier 2019 }}^{27}$ : Cohort study with acute lymphoblastic leukemia patients (under age 18) and their parents treated at 1 cancer center ( 22 families enrolled, 20 families completed all time points) to determine parent and patient experiences with SCP

- Res: SCP based on Passport for Care and ASCO SCP guidelines

- Res+OC: $90 \%$ of parents were highly satisfied with process of receiving SCP and layout, length etc.

- Res+Pt Use: $95.75 \%$ of parents intended to share SCP with someone at T2, but only $60.9 \%$ had done so at T3; only $35 \%$ of those with a PCP had shared it with them by T3. No parents had shared with school, and though $52.2 \%$ at T2 said that they would share with other HCPs, at T3, only $8.7 \%$ had done so. A few families reported that the SCP prompted them to find an SCP.

- Res+Pt F/B: Between enrollment and follow up there was a shift to wanting SCP provision during, rather than at the end, of treatment. Parents wanted oncologist to deliver SCP; $26.1 \%$ parents at $\mathrm{T} 2$ and $8.3 \%$ at $\mathrm{T} 3$ said that the provider used medical terms they didn't understand; only $38.5 \%$ of patients felt all questions were answered.

\section{Reports From Survivors}

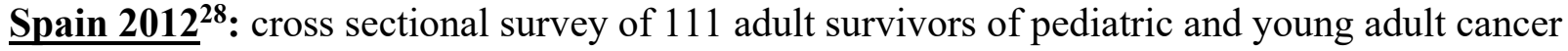
from Memorial Sloan-Kettering Cancer Center Adult Long-Term Follow-Up Program assessing retention, understanding, concerns, preferences 1-6 weeks after receipt of SCP

- Res: one-page SCP (treatment summary + f/u care plan); 1-6 weeks after receipt, 95\% had the document (55\% on hand, $40 \%$ at home in a personal file); $95 \%$ wanted online or wallet-card version of SCP; $80 \%$ said SCP contained sufficient content, $20 \%$ too little; extra info requested on SCP: meds/allergies, all health care providers with contact info, 
all surgeries, current late effects status, tests with dates/results, mental health recs/resources, all dosages of cancer treatment

- Model: 57\% had PCP outside MSKCC, 23\% were new pts who never had SCP before

- $\operatorname{Res}+$ Pt F/B: $95 \%$ understood everything on treatment summary, $40 \%$ found extremely and 43\% moderately valuable; $95 \%$ thought SCP helpful in understanding plan for their care, and important link between health care providers; of 30 who visited an outside provider since getting SCP, $1 / 3$ gave the provider a copy of the form and $44 \%$ to someone in their personal circle

- Res+Pt F/B: SCP as a whole caused concern for $14 \%$, screening recommendations concerning for $14 \%$, potential late effects caused concern for $28 \%$ (moderate/extreme worry in $13 \%$ ), $4 \%$ had mod to extreme worry about cancer, $3 \%$ with coping with survivorship, $6 \%$ with sexual/reproductive worry

- Res+Pt Char: new pts reported learning new info from SCP compared to return pts (68\% vs $39 \%$ )

- Pt Char+Pt F/B: lymphoma dx and older age increased odds of general health worry (lymphoma survivors were significantly older than non-lymphoma survivors though)

- Pt Char+Pt F/B: being male and younger associated with sexual/reproductive worry

- Res+Pt Char: being older, more time since diagnosis, and less understanding of treatment summary associated with seeking more info on treatment summary (bivariate); after adjusting for age, gender, time since $\mathrm{dx}-->$ less understanding of treatment summary was associated with seeking more info on treatment summary

- Res+Pt Char: Those who shared SCP not different significantly from those who did

Shay 2017 ${ }^{29}$ : survey of $\mathrm{n}=1395$ AYAs $(11 \%$ diagnosed $<21)$ about SCPs (treatments summary + instructions for followup care)

- $\operatorname{Res}+$ Pt F/B: SCP and instructions significantly associated with lower odds of reporting unmet needs around information about late effects of treatment

- $\operatorname{Res}+\mathbf{P t}$ F/B+OC: instructions for followup care decreased unmet needs related to fertility

- Pt Char+Pt F/B: factors associated with higher odds of unmet fertility information needs included being female, younger age at diagnosis, having a diagnosis of lymphoma or testicular cancer, and currently seeing an oncologist

- $\operatorname{Res}+\mathbf{P t}$ F/B: both receipt of a written treatment summary and instructions for followup care were associated with lower odds of reporting unmet needs around recurrence concerns

- Pt Char+Pt F/B+Pt Use: female survivors and those currently employed were more likely to report unmet needs around cancer recurrence concerns; survivors who were currently seeing a primary care physician and who were younger at the time of the survey were less likely to report unmet needs about cancer recurrence concerns

- Res+OC (family risk of cancer): In unadjusted models, but not adjusted models, survivors who reported receipt of a written treatment summary and instructions for followup care were less likely to report unmet needs around concerns about family risk of cancer.

- Pt Char+OC (family risk of cancer): factors associated with unmet needs around family risk of cancer included being female, having lower levels of education, and being 
married. Survivors with a history of leukemia, lymphoma, or other cancers had lower odds of reporting unmet needs around family risk of cancer

\section{Other Resources}

\section{Prospective Studies}

$\underline{\text { Hudson 2002 }}^{\mathbf{3 0}}$ : longitudinal, controlled prospective trial to determine if a multi-component riskcounseling intervention could induce change in 272 (251 evaluated at both time points) childhood cancer survivors' health knowledge, health perceptions, and practice of healthprotective behaviors.

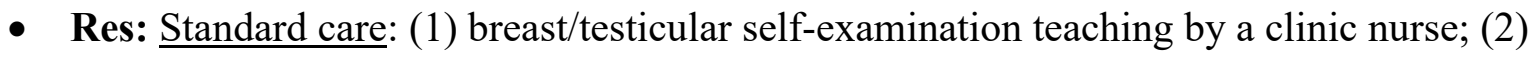
targeted late effects screening based on clinical history and treatment exposures; (3) a thorough clinical assessment by a clinic physician or NP; and (4) late effects risk counseling; Intervention arm: standard care plus: (1) distribution and discussion of written After Completion of Therapy (ACT) clinical summary; (2) health behavior training in health goal chosen by the survivor; (3) health goal commitment to practice chosen behavior during ensuing year; and (4) telephone followup at 3 and 6 months to reinforce behavioral training. The Health Belief Model was used to guide the development of the intervention and identify factors that could potentially influence behavioral change. Eligibility for ACT clinic: remission a least 5 years from diagnosis, have been off therapy for 2 years, and lack of chronic medical problems requiring frequent medical intervention by the St. Jude staff

- Res+Pt Char+Pt F/B: In a sub-group analysis, there was a significant difference between males and females in change scores for health knowledge in the Intervention Group, with the females in the cohort generating greater improvement in knowledge. | (from Discussion): Although gender difference on knowledge were statistically significant, the small increase in knowledge among females may not represent a clinically significant change in acquired health information. Nonetheless... risk counseling activities may need to account for the gender composition of the target population.

- Res+Pt F/B+Pt Pract: No significant differences in change scores (from baseline to 12month followup) between the Intervention Group and Standard Care for: (1) health knowledge, (2) health perceptions of susceptibility to health risks, (3) barriers to protective health actions; (4) benefits of protective health actions; or practices.

- Res+Pt F/B+Pt Pract: The limited effectiveness of our intervention suggests that a brief, broad-based risk counseling approach may not be sufficient to achieve a substantial long term change in knowledge, health perceptions, and health practices. Our intervention was designed to be brief, administered in a single session with telephone followup, and easily delivered in a clinical setting. A more intensive intervention conducted over several sessions with revisions of content and method of delivery may be necessary to enhance the impact of our program. [author speculation on context-mechanism-outcome hypothesis]

$\underline{\text { Steele } 2013}^{31}$ : Random assignment of survivors to receive two types of newsletters with the aim to assess their effectiveness in increasing medical followup in pediatric cancer survivors at risk of selected treatment complications (breast cancer, heart disease, and osteoporosis); 5.661 
survivors participating in the CCSS who were at least 25 years of age (as of July 2, 2001) and completed both the baseline and followup (FU-2) survey

- Res: (1) a newsletter featuring brief health risk information or (2) a newsletter including an insert providing more comprehensive health risk information.

- $\operatorname{Res}+P t$ F/B: Women at risk for osteoporosis who received the newsletter insert were more likely to have discussed their risk with a doctor than those who only received the brief information (although significance was only borderline);

- Pt F/B+Pt Use+Prov Pract: Discussing one's cancer-related risk with a doctor is the strongest predictor of getting screened for late effects.

- Res+Pt Use+Prov Pract: A physician's access to the survivor's cancer treatment summary also significantly predicted the respondent's receipt of screening for the targeted health risk.

- $\operatorname{Res}+\operatorname{Res}+\mathbf{P t}$ Use+Pt F/B: No difference between groups in terms of access to a treatment summary, medical followup, discussion of childhood cancer health risks, and medical screening for the targeted health behaviors.

- Res+Pt F/B+Pt Use+Prov Pract: More detailed content in a newsletter had minimal effect on recommended screening. However, survivor's discussion of cancer-related risks with one's doctor significantly influenced participation in health screening. These results highlight the integral role of communication in health behavior.

- Res+Pt Char: Substantially more women (72\%) than men (59\%) reported reading the newsletter. Analysis of the sample as a whole indicated that in addition to gender and older age, higher education and being currently married were all moderately but significantly correlated with higher newsletter readership.

- Res: The relatively weak intervention dose (one targeted, not tailored page of information in a 2-year period) may explain the null finding. Tailored information seems to influence desired outcomes more consistently than targeted information. However, even with more intensive interventions, effects are persistently small. [author speculation on context-mechanism-outcome hypothesis]

- Pt F/B+Pt Use+Prov Pract: Discussing cancer-related risks with one's doctor substantially increased the likelihood of undergoing recommended screening tests. These findings identify behavioral targets that can be modified through intervention (e.g., treatment summary for each patient) [author speculation on context-mechanism-outcome hypothesis]

\section{Surveys}

Nathan 2011 $^{\text {32}}$ : Survey of 56 pediatric hem/onc fellowship training programs and their training program directors in the United States and Canada to evaluate their resources and capacity for survivorship education, and to explore the barriers and opportunities for the optimal provision of survivorship-centered training.

- Res+Prov Char: 86\% require attendance at long-term followup clinic by pediatric hematology/oncology fellows. Of the 37 programs that required pediatric

hematology/oncology fellows to attend survivor clinics, 32 reported the length of the survivorship rotation. Twenty-one required rotations of 4 weeks or less, 5 required 8- to 12-week rotations, and 6 required rotations of greater than 12 weeks. Graduate medical trainees who rotate through the LTFU clinics include medical students in $21(46.7 \%)$ 
institutions, pediatric residents in 25 (55.6\%), family medicine residents in $2(4.4 \%)$, medical hematology/oncology fellows in $4(8.8 \%)$, and radiation oncology fellows in 8 $(17.7 \%)$.

- Res+Prov F/B: Thirty-six programs (80\%) provide didactic lectures on survivorship. These sessions are mandatory for pediatric hematology/oncology fellows at 26/36 (72.2\%) institutions, for pediatric residents at 5 institutions (13.9\%), and for family medicine residents at 1 institution; Only $2(4.4 \%)$ considered the training "very effective," while $20(62.5 \%)$ considered it "somewhat effective," $8(25.0 \%)$ "a little bit effective," and 5 (15.6\%) "not very effective."

- Res+Prov F/B: Majority of programs require hem/onc fellows to have experience assessing survivors (M-C); half of the programs require 4 weeks or less training (M-O); Time noted as the biggest barrier to providing training $(\mathrm{C}-\mathrm{O}) ;$; ".. LTFU clinic directors feel that there is insufficient emphasis on survivorship training as part of the fellowship curriculum." (C-O) [author speculation on context-mechanism-outcome hypothesis]

\section{Adult Survivors of Adult Cancer}

\section{Models of Care}

\section{Prospective Studies}

$\underline{\text { Nielsen 2003 }}^{\text {33 }}$ : RCT in 248 consecutive cancer patients (August - December 1998) recently transferred to the Department of Oncology at Aarhus University Hospital (Denmark) to determine the effect of a shared care program and to assess patients' reports on contacts with their GP; patients completed questionnaires at three time points.

- Model: Shared care program that had three elements: (1) knowledge transfer (discharge summary letters), (2) communication channels (providing the names / phone numbers of doctors and nurses responsible for the patient), (3) active patient involvement (oral / written information about the information package to their GP). The intervention was compared with the normal procedure of the Department of Oncology (i.e., no standard procedures for informing the GPs about newly diagnosed cancer patients).

- Model+Pt F/B+Pt Char: The shared care program had a positive effect on patient evaluation of cooperation between the primary and secondary healthcare sectors. The effect was particularly significant in men and in younger patients (18-49 years) who felt they received more care from the GP and were left less in limbo. Young patients in the intervention group rated the GP's knowledge of disease and treatment significantly higher than young patients in the control group.

- Model+Pt Use: The number of contacts with the GP was significantly higher in the intervention group.

- Model+OC: The EORTC quality of life questionnaire and performance status showed no differences between intervention and control.

- Model+Prov F/B: The discharge summary letters were the most difficult part of the program as they interfered with the daily routine of the doctors.

- Model+Prov F/B: "It is essential to have an innovator when performing shared care programs across borders in a healthcare system who has connections with both the 
primary and the secondary sectors. The innovator must be prepared to invest time and energy in getting the project accepted in both settings" (CY: innovator = boundary spanner); Challenge to improve interpersonal communication between doctors in different settings remains; further research is required into cultural barriers to communication in the healthcare system

$\underline{\text { Ramirez 2019 }}^{34}$ : RCT to compare culturally tailored navigation $(\mathrm{n}=60)$ to navigation $(\mathrm{n}=60)$ in Stage 1-3 Latina breast cancer survivors; baseline and 6-month follow up after intervention

- Model+Res+OC: Significant intervention effect for FACT-G ( $p=.02)$, FACT-B ( $p=.01)$, Social well-being $(\mathrm{p}=.04)$, Emotional well-being $(\mathrm{p}=.03)$, Functional well-being $(\mathrm{p}=.01)$, $\mathrm{BC}$ subscale ( $\mathrm{p}=.007$ ), but not physical well-being; control QoL got worse between baseline and 6-months, intervention improved

Grunfeld 1999 ${ }^{35}$ : Randomized controlled trial of 296 women with breast cancer from 2 district hospitals in UK comparing followup by specialist group vs. GP

- Models+OC: higher patient satisfaction expressed by patients receiving follow up with GPs. Patient satisfaction increased more among GP patients from baseline to mid trial. Patients in GP group were more satisfied with continuity of care and access to physician.

Joubert 2006 $^{\mathbf{3 6}}$ : RCT in 97 patients discharged from acute stroke unit in Australia to evaluate health outcomes for a shared care model vs "treatment as usual" post-discharge care

- Models+Pt Use: Percentage of patients reaching the (recommended) total cholesterol of $5.18 \mathrm{mmol} / \mathrm{L}$ was greater in the intervention group $(\mathrm{p}=0.02)$

- Models+Pt Use: Exercise participation, increased over the twelve-month period in the intervention group compared to the control group $(\mathrm{p}=0.048)$

- Models+OC: No significantly different rates of depression between arms $(\mathrm{p}=0.06)$

\section{Reports From Survivors}

$\underline{\text { Dyer 2016 }}^{37}$ : cross-sectional study of 441 long-term survivors (average age 54 years) of Bone Marrow Transplant (BMT) in New South Wales (NSW), Australia who were 17 or older when they had BMT to identify preferences for long-term care

- Models: $275(62.3 \%)$ preferred a single provider for their primary transplantation followup (ie, general practitioner [GP] alone, local hematologist [LH] alone, or transplantation physician [TP] alone). An additional 149 (33.8\%) preferred a combination of providers and $17(3.8 \%)$ indicated no preference. The majority $(44.9 \%)$ of those surveyed indicated a preference for their TP alone to be primarily responsible for their LTFU care. The second preferred option included a combination of TP and LH (14.2\%), followed by LH alone (13.1\%), GP and LH and TP (7.7\%), GP and TP (7.7\%), GP alone (4.3\%), or GP and LH (4.1\%). Of the 441 patients surveyed, $329(74.6 \%)$ indicated a followup preference that included a TP, $173(39.2 \%)$ included a LH, and $105(23.8 \%)$ included a GP. In terms of location, 328 of 441 (74\%) BMT survivors reported a preference for followup at transplant center (TC) alone or in combination with other provider locations, such as satellite clinics linked with a TC or telemedicine services administered by the primary TC. Of the entire cohort, TC alone was the preferred option by $121(27.4 \%)$ and LH practice alone was preferred by $57(12.9 \%)$. Twenty-one (5\%) 
indicated a preference for follow- up with GP practice alone, $18(4.1 \%)$ for telemedicine alone, and 17 (3.8\%) for satellite clinic alone.

- Models+Pt Char+Pt Needs: Variables associated with an increased preference for TC or TC-linked followup included being in a married/de facto relationship (OR, 1.67; P 1/4 $.04)$ and sexual dysfunction (OR, 2.15; P 1/4 .006). Those in full-time or part-time employment indicated a decreased though nonsignificant preference for followup with TC or TC-linked services (OR, .67; P 1/4 .08). On multivariable analysis, no variables showed a significant association with a preference for TC or TC-linked followup. Those reporting increased severity of GVHD symptoms showed a trend towards increased preference for TC or TC-linked followup (AOR, 1.16; 95\% CI, .99 to 1.36; P 1/4 .06) and those in full- or part- time employment showed a trend towards decreased preference for TC or TC-linked followup (AOR, .44; 95\% CI, .19 to 1.03; P 1/4 .06). Those preferring LTFU in satellite clinic settings were more likely to be from a middle/high income group (OR, 1.98; 95\% CI, 1.20 to 3.33 ; P 1/4 .005) and to have a higher educational status (OR, 2.07; $95 \%$ CI, 1.23 to 3.49 ; P 1/4 .003), defined as partial or complete attainment of a university qualification.

- Models+Pt Needs: No significant differences in nontransplantation-related chronic disease, cancer, or psychological morbidity were observed in those who preferred LTFU in a TC or TC-linked service. Self- reported chronic graft vs host disease (cGVHD) symptoms described as moderate/severe were significantly lower in those preferring followup in a satellite clinic setting (OR, .55; 95\% CI, .29 to 1.0; P 1/4 .04), and median self-reported current GVHD severity scores were significantly lower (P 1/4 .05). Sexual dysfunction was significantly higher in those expressing a preference for satellite clinic followup (OR, 2.61; 95\% CI, 1.46 to 4.74; $\mathrm{P}<.001)$.

- Models+Pt Use: No significant difference in cancer screening was observed between survivors preferring LTFU with TC or TC-linked service, with the exception of Pap smear uptake in females. Females preferring followup through TC or TC-linked services were less likely to report having had a post-transplantation Pap smear (OR, .50; 95\% CI, .22 to 1.06: P 1/4 .05). After adjusting for potential confounders including age, educational status, residential location, marital status, GVHD severity, and sexual dysfunction, no significant difference was observed (AOR, .19; 95\% CI, .03 to 1.25; P 1/4 $.08)$.

$\underline{\text { Viscuse 2019 }}^{38}$ : survey to assess the impact of survivorship clinic (SC) use on patient centered outcomes in 154 adult lymphoma survivors (65 who attended survivorship clinic and 89 who did not) treated at Mayo Clinic

- Models+Res: Patients who went to SC were more likely to receive SCP

- Models+Pt F/B: Patients who went to survivorship clinic (SC) were more likely to at least "somewhat recall discussing preventative health, lifestyle improvements, nutrition and exercise $(p<0.01)$, less likely to need at least some more information on long-term side effects and decreasing risk of having cancer again, or sexual function (all $\mathrm{p}<0.05$ ). More in SC group were confident to get information on cancer if needed $(p<0.05)$

- Pt Char+OC: Younger patients in SC group had better overall health perception

- Pt Char+OC: Among HL patients in SC group, physical QoL was higher - not significant once adjusted by age. 
- Models+OC: No difference between SC group and non-SC group in overall health, physical QoL, mental QoL and distress

- Model+Res: "It's not the piece of paper - it is the conversations initiated as a result of the piece of paper that matters" "comprehensive survivorship care, delivered within a specialized SC in our study, improves patient outcomes and does not increase distress of worsen emotional QOL"

$\underline{\text { Mao 2009 }}^{39}$ : Cross-sectional survey to describe perceptions of PCP-related survivorship care from 286 post-menopausal breast cancer survivors (BCSs) seen in an outpatient breast oncology clinic at UPENN who had a PCP

- Model+Pt F/B: BCSs rated PCP-related survivorship care as 65 out of 100. The lowest rated item was interspecialty communication; only $28 \%$ felt that their PCPs and oncologists communicated well.

- Model+Prov Pract: Most items related to specific cancer survivorship care (symptom diagnosis and management, followup, and surveillance for late effects of cancer therapies) had lower endorsements $(41 \%, 50 \%$, and $59 \%$, respectively); areas of PCPrelated care most strongly endorsed: general care (78\%), psychosocial support (73\%), and health promotion $(73 \%)$

- Model+Pt Char+Pt F/B: In a multivariate regression analysis, nonwhite race and level of trust in the PCP were significantly associated with higher perceived level of PCPrelated survivorship care

- Model+Pt F/B+Prov F/B: Survivors' recommendations for enhancing primary care delivery of survivorship care:

- Teaching PCPs about issues relevant to BCS

- Improving communication between oncologists and PCPs

- Teaching themselves about how to communicate with their PCPs and oncologists so that the care could be cohesive (note: this strategy was more likely to be endorsed by nonwhite and less educated BCSs)

- Developing a primary care clinic specific for BCSs

- Model+Pt Char+Pt F/B: Nonwhite (predominantly black) patients rated survivorship care delivery by PCPs higher and wanted to be taught strategies for communicating better with PCPs and oncologists. They also preferred having a primary care-based survivorship clinic. We hypothesize that nonwhite survivors may rely on PCPs more than specialists in chronic disease management because of access, communication, and cultural issues. Research is needed to evaluate interventions to facilitate communication between oncology and PCPs for survivorship care. [author speculation on context-mechanismoutcome hypothesis]

\section{Reports From Providers}

Mani 2020 $^{\mathbf{4}}$ : cross sectional survey of 86 PCPs across 2 health systems (Cleveland Clinic and Mayo Clinic in AZ) regarding practice patterns and barriers to caring for heme cancer survivors

- Prov Pract+Prov F/B: PCPs who had cared for greater number of heme cancer survivors in the last 5 years more likely to report receiving periodic updates from oncologist

- Prov Pract+Prov F/B: as number of survivors increased in past 5 years, proportion of PCPs who often/always had issues with inadequate resources or inadequate communication from oncologist decreased; PCPs who cared for greater number of survivors past 5 years more confident in managing cancer-related medical issues, cancer 
surveillance, secondary cancer screening, and general medical issues (multivariate analysis)

- Prov Pract+Prov F/B: as \# patients seen/week increased, proportion of PCPs often/always having difficulty transferring patient care responsibilities and lacking awareness of screening/prevention guidelines dropped

- Prov Pract+Prov F/B: higher percentage of PCPs with teaching responsibilities were more confident in dealing with general medical issues (also in multivariate analysis)

- Prov Pract+Prov Pract: PCPs who had cared for greater number of heme cancer survivors in the last 5 years more likely to report discuss screening/late effects with pts, and more likely to prefer being involved in cancer surveillance

- Prov Pract+Prov Pract: higher percentage of PCPs with teaching responsibilities preferred managing general medical issues, general cancer screening, management of psychosocial issues

- Prov Char+Prov F/B: more years since residency associated with higher confidence in managing cancer-related medical issues, cancer surveillance (including in multivariate analysis) and decreases in proportion of PCPs who often/always lack awareness of clinical/psychosocial needs of survivors and awareness of screening/prevention guidelines

- Prov Char+Prov F/B: $63 \%$ of PCPs had no difficulty transferring patient care responsibilities with oncologist

- Prov Pract: $45 \%$ discussed cancer surveillance, secondary cancer screening, or late effects

- Prov F/B: barriers = lack of resources to facilitate survivor care (69\%), lack of awareness of psychosocial needs and insufficient time (65\% for each), lack of awareness of guidelines $(55 \%)$, lack of clarity by patient on which provider to call for health issue (77\%), pt prefers fu with oncologist $(66 \%)$, inadequate communication with oncologist (52\%), insufficient reimbursement (43\%)

- Prov Char+Prov Pract: categories of care that PCP preferred to handle (vs oncologist/shared with onc/other consultant), only general medical issues (84\%), general cancer screening (73\%), and psychosocial (49\%) -- rest were $20 \%$ or less (cancer related medical issue, cancer surveillance, secondary cancer screening, fertility)

- (experience caring for survivors) the more PCPs were involved in caring for survivors (more survivors seen or longer time in practice) the more comfortable they were doing it and the fewer obstacles they perceived. [author speculation on context-mechanismoutcome hypothesis]

Stephens 2021 ${ }^{41}$ : cross sectional survey of 29 of 100 eligible PCPs at an academic center regarding confidence and care coordination of older breast cancer survivors

- Prov Char: PCPs mean age 43.5, 13.5 years of clinical experience

- Prov Char+Prov F/B: $28 \%$ not confident $/ 17 \%$ somewhat confident in addressing late effects of cancer treatment

- Prov Char+Prov F/B: $24 \%$ not confident/somewhat confident addressing chronic comorbidities in pts

- Prov Char+Prov F/B: 3\% reach out to patient's oncologist all of the time, 52\% reach out little/none of the time. 
- Prov Char+Prov F/B: 48\% answered no to regularly addressing patient's followup clinical visits with oncologists

$\underline{\text { Worster 1996 }}^{\mathbf{4 2}}$ : Survey to identify experience and willingness of family physicians $(n=154)$ in Ontario to take on followup care for breast cancer survivors

- Model+Prov Char+Env: 90\% said would accept responsibility of followup care; $77 \%$ said appropriate for FPs to take on followup care for stage 1 breast cancer; $3.9 \%$ said not appropriate; distance to cancer clinic did not seem to influence responses

$\underline{\text { Brennan 2010 }}^{\mathbf{4 3}}$ : cross sectional survey of attitudes of 161 practitioners in Australia (breast surgeon, med onc, rad onc, breast physicians, breast care nurses) who care for breast cancer survivors

- Model: 36\% specialists discharged pts from their care after 5 years, 56\% after 10 years

- Prov Char+Prov Pract: gaps in psychological care, social care and general health care from specialists

- Prov Char+Prov Pract: specialists felt cancer recurrence was main role (99\%); fewer (67 and 29\%) felt psychological and social care were part of their role - none felt general health care was their main role in $\mathrm{f} / \mathrm{u}$ care

- Res+Prov Char: 51\% specialists, $83 \%$ breast physicians, and 92\% breast care nurses thought SCP would improve care for survivors

- Model+Prov F/B: specialists have an interest in sharing care with other providers - but 96\% specialists felt that in order to share care, PCPs need more training

- Prov Char+Prov Pract: $10-20 \%$ of specialists felt they spent too much time in $\mathrm{f} / \mathrm{u}$ care

$\underline{\text { Dixit 2020 }}^{44}$ : Cross-sectional survey to assess the knowledge and attitudes of 110 PCPs (MD, NP, PA) and preferences for care models for breast and colon cancer survivors in the 12 primary care clinics of the San Francisco Health Network (safety net)

- Models+Prov F/B: PCPs felt that communication from the oncologist could be significantly improved when transitioning the patient to primary care

- Models+Prov F/B: About half of PCPs strongly/somewhat agreed (vs. strongly/somewhat disagreed) that PCPs have the knowledge needed to provide followup care related to breast (50\%) and colon cancer (54\%).

- Models+Prov F/B: $33 \%$ of providers reported receiving any survivorship training

- Models+Prov F/B: The most preferred model for survivorship care was shared care model (40\%).

- Models+Prov Pract: Monitoring of long-term adverse effects of treatments was less well-recognized than surveillance practices, $12 \%$ of PCPs correctly identifying neuropathy as adverse effect of oxaliplatin; $44 \%$ recognizing cardiotoxicity as adverse effect of Doxorubicin (Adriamycin), and 28\% recognizing paclitaxel-induced neuropathy.

- Models+Res+Prov F/B: $20 \%$ of PCPs reported that they often or always received a comprehensive cancer treatment summary from oncology

Forsythe 2012 $^{\mathbf{4 5}}$ : Survey of Physician Attitudes Regarding the Care of Cancer Survivors (SPARCCS) in 1,130 oncologists and 1,021 PCPs who care for breast or colon cancer survivors; this analysis focuses on psychosocial care 
- Model+Prov Char+Prov Pract: both reported involvement with psychosocial care; greater percentage of PCPs reported involvement in health promotion ( $87 \%$ vs $79 \%)$, sole provision of health promotion (47 vs 31); oncologists were more likely than PCPs to report shared provision for psychologic care, health promotion, and psychosocial care overall, whereas PCPs were more likely to report shared provision for management of physical symptoms and sole provision for health promotion and psychosocial care overall

- Model+Prov Char+Prov Pract: oncologists' and PCPs' perceptions of who delivers specific aspects of care differ substantially; each frequently endorsed his or her own provider group as involved in this care and rarely endorsed the other as usual providers. [turf issue? Acceptance of shared care model?]. [author speculation on contextmechanism-outcome hypothesis]

Numico 2014 ${ }^{46}$ : Survey to assess attitudes and feelings of Italian oncologists ( $\left.\mathrm{n}=91\right)$ in reference to creating more efficient models of survivorship care delivery.

Basically describes Italian oncologists description of their role in providing survivorship care to adult survivors of adult cancers. [author speculation on context-mechanism-outcome hypothesis]

- The final part of the survey assessed the meaning of follow up as perceived by oncologists (Figure 3). Only $6 \%$ and $14 \%$ of the oncologists feel that follow up has an important role in reducing mortality or anticipating the detection of recurrences in breast cancer. These figures grow to $31 \%$ and $47 \%$ for colorectal cancer. An important role for management of late toxicities is perceived by $26 \%$ for breast cancer and $17 \%$ for colorectal cancer. Management of comorbidities is perceived a major role in survivorship care by $24 \%$ for breast and $17 \%$ for colorectal cancer. The educational role is considered important in $36 \%$ and $32 \%$ while the psychological and supportive role for $44 \%$ and $37 \%$.

Bober 2009 ${ }^{47}$ : Cross-sectional survey to detail practice patterns, attitudes, and challenges in caring for long-term cancer survivors among community and academic based general internal medicine physicians in Denver, Colorado ( $\mathrm{n}=227)$.

- Prov Char+Prov Pract: PCPs in practice $>10$ years were 7 times more likely to deliver multidimensional care $(\mathrm{OR}, 7.17 ; 95 \% \mathrm{CI}, 1.7-30.9[\mathrm{P}<.008])$ than PCPs with $<10$ years in practice.

- Prov Char+Prov F/B: $47.9 \%$ of PCPs reported feeling unprepared to evaluate the longterm effects of adult cancer survivors and $49.8 \%$ reported feeling unprepared to manage these long term.

- Prov Char+Prov F/B+Res: Commonly endorsed barriers to delivering survivorship care were: lack of standards for survivorship care (52.5\%); inadequate preparation/formal training around survivorship (47.2\%); and limited access to mental health referrals for cancer survivors $(45.7 \%)$.

- Prov Char+Prov Pract: $37.3 \%$ of PCPs reported never or only rarely screening for medical late effects, $62 \%$ of PCPs reported never or only rarely addressing issues of sexual dysfunction, and $33 \%$ reported never or rarely addressing mental health issues.

- Prov Char+Prov F/B+Res: $98 \%$ of PCPs would like to receive additional descriptions of patients' previous cancer treatments, including therapeutic exposures, information regarding short-term complications, and individualized guidelines for care. 
$\underline{\text { Cheung 2012 }}^{\mathbf{4 8}}$ : Surveyed primary care providers (PCPs) $(\mathrm{n}=938)$ and oncologists $(\mathrm{n}=1088)$ to assess how physician attitudes toward and self-efficacy with cancer followup affect preferences for different cancer survivorship models

- Models+Prov F/B+Prov Char: Most oncologists were confident in their knowledge regarding following patients for cancer recurrences, and managing cancer and its longterm and late effects (80 and $70 \%$, respectively), PCPs had lower self-efficacy in these areas (34 and $19 \%$, respectively; both $\mathrm{p}<0.001$, comparing PCPs vs. oncologists)

- Models+Prov F/B+Prov Char: Most PCPs reported they were better able to provide routine cancer followup (57\%), detect and work-up recurrent cancers $(74 \%)$, and offer psychosocial support to survivors (50\%). However, only $32 \%$ felt they were able to assume primary responsibility for survivorship care.

- Models+Prov F/B+Prov Char: Majority of cancer specialists did not feel that PCPs were adequately supported or trained to offer routine cancer followup (78\%), detect cancer recurrences (62\%), provide psychosocial support (92\%), or take on the principal role for survivorship care $(87 \%$; all $\mathrm{p}<0.001)$.

- Model+Prov Char: PCPs and oncologists who were 40 years or older were significantly more likely to prefer an oncologist-based model instead of a PCP/shared care or specialized survivor clinic model than those under 40 years, after controlling for covariates (both overall $\mathrm{p}<0.05$ ).

- Model+Prov F/B+Prov Char: PCPs already involved with cancer surveillance (43\%) were more likely to prefer a PCP/shared care than oncologist-based survivorship model (OR, 2.08; $95 \%$ CI, 1.34-3.23).

- Model+Prov F/B+Prov Char: Proportion of oncologists (87\%) did not feel that PCPs should take on the primary role of cancer followup.

$\underline{\text { Buriak 2015 }}^{\mathbf{4 9}}$ : Cross sectional survey of 1809 providers who took online course [physicians (n $=229)$, NPs $(n=213)$ and RNs $(n=1367)]$ to assess predictors of intention to provide survivorship care guided by the theory of planned behavior (TPB)

- Res: Educational intervention to enhance survivorship expertise among PCPs through a "cancer survivorship primer containing epidemiological information, patient cases, guidelines for clinical followup, and reference materials for delivery through the WebMD platform

- Associated with intent to provide survivorship care:

○ Model+Prov Char: physicians less likely than NP/RN; 6-10 years in practice more likely (11-20 years approached significantly more likely); provider survivorship status not associated

- Model+Prov F/B: perceived presence of barriers less likely

- Res+Prov F/B+Prov Char: A smaller percentage of RNs (39.73\%) perceived barriers to survivorship care than in either the physicians $(51.53 \%)$ or NP $(49.77 \%)$ groups. Most common barrier for all groups: lack of survivorship care plans and treatment summaries; lack of education and training was 2nd for NPs and RNs, 3rd for physicians; lack of insurance coverage and lack of standard survivorship model also reported by $>10 \%$ in all groups; coordination of care between PCP and oncology and lack of EMR less often cited as barriers; $2 \%$ identified no barriers 
- Prov F/B+Prov Pract: theory of planned behavior with focus on knowledge, attitudes, and perceived barriers in relation to intent to deliver survivorship care. [author speculation on context-mechanism-outcome hypothesis]

- Prov Char+Prov F/B: observation that RNs did not perceive as many barriers as NPs or physicians may be attributable to lower responsibility for overall patient care; physician and NP alignment on barrier recognition could arguably be due to similarities in accountability for and volume of patient care. [author speculation on context-mechanismoutcome hypothesis]

- Res+Prov F/B: recommend extending this educational strategy to include video vignettes and multimedia presentations featuring midcareer provider champions who have successfully incorporated survivorship care into their daily practice and can share experiential examples of how they overcame specific barriers to providing this care; these firsthand examples should be accompanied by direct links to current survivorship plan templates and guidelines for surveillance and followup in order to provide practical, "how-to" resources to help less experienced clinicians develop confidence in their capacity to perform competently in this arena. [author speculation on contextmechanism-outcome hypothesis].

Chubak 2012 ${ }^{\mathbf{5 0}}$ : Interviews with 40 of 48 approached clinical leaders, administrators, and providers in oncology, primary care, nursing administration, and specialty care from 10/14 sites of the Cancer Research Network to find out about survivorship care in integrated health care delivery systems

- Models+Prov F/B: Interviewees reported belief that oncology and PCPs are jointly responsible for the care of cancer survivors and highlighted the role of electronic medical records in coordinating and sharing care; highlights evidence gaps, including value, effectiveness, and cost-effectiveness of different care models, group visits, and selfmanagement programs; financial costs and benefits of formalizing survivorship care (including reimbursement issues)

- Res: Many respondents were not familiar with survivorship care plans

- Models+Prov Char+Prov F/B: Only 2 sites had formal cancer survivorship programs in place (1 nurse-led program, 1 physician assistant-led consultative service), both with dedicated funding and a provider champion; 2 were developing programs

- Res+Prov F/B: Some use and some lack of awareness of survivorship care plans and planning; some mention of guidelines; provided some non-survivorship-specific tools such as educational materials and seminars and support groups

\section{Other Study Designs}

Etim 2006 $^{51}$ : Retrospective cohort using SEER-Medicare to evaluate receipt of mammography in 3828 breast cancer survivors followed by generalist physicians, specialists, or both

- Prov Char+Pt Use: Patients who saw generalists only, receipt of mammography was $57.7 \%, 55.1 \%$ and $54.2 \%$ respectively in followup years $1-3$; in shared care patients was $84.0 \%, 81.0 \%$ and $78.6 \%$.

- Prov Char+Pt Use: In a multivariate logistic regression model, women receiving shared care had substantially greater mammography use than others, OR 2.13 (95\% CI: 1.74, 2.58) 
- Pt Char+Pt Use: Stage II disease and patients of African American race were significantly less likely to receive followup mammography during the first and third followup years.

- Model+Pt Char: "patients followed up only by generalists had greater comorbidity, resided in less affluent and less urban areas, and received different treatments from those followed up by specialists"

$\underline{\text { Worster }}$ 1995 $^{\mathbf{5 2}}$ : Chart review to assess how frequently family physicians were involved in posttreatment followup care of their patients with stage I breast cancer $(n=183)$ in Canada

- Model+Pt Char+Env: PCP involved in followup care in 12\% for cases of surgery and radiation and 29\% surgery. PCPs infrequently involved and less so for those receiving radiation; no differences based on distance of patient to cancer clinic

$\underline{\text { Choi 2020 }}^{53}$ : Description of a survivorship program and survivors who attended the clinic

- Model: Primary Care for Cancer Survivor Clinic; patients are seen by 1 of 6 general internists with interest/expertise in cancer survivorship; providers also see non-cancerrelated longitudinal patients; primarily funded by provider relative value units; providers hold monthly meetings to review clinical operations, provide updates on cancer survivorship-related topics, and discuss ongoing research initiatives

- Model+Env: 2 general internal medicine practices (1 urban hospital-based, 1 suburban ambulatory)

- Model+Prov Pract: The visits address cancer surveillance, late and long-term effects, preventive health including lifestyle counseling, comorbidities, and/or palliative care as appropriate, and initial visits are guided by a template; services include medication prescriptions, counseling, and referrals to specialists and ancillary services

- Model+Res: Collated a shared list of medical providers and community resources

- Model+Prov F/B: Describes the high quality of communication between the clinic and oncology providers, which is perceived as valuable; discusses the value of embedding survivorship care in primary care, where survivorship issues are seen as part of the entirety of the patient's health; RVUs are higher for PCCS clinic patients, supporting the clinic's economic viability; had more demand for longitudinal than consultative care, creating capacity issues. [author speculation on context-mechanism-outcome hypothesis]

\section{Combined Model and Resource (e.g., SCP)}

\section{Prospective Studies}

$\underline{\text { Mayer 2016 }}^{54}$ : Pilot, randomized controlled trial comparing SCP transition visit with oncology NP vs SCP transition visit+PCP followup visit in 34 adult cancer survivors in community cancer center

- Pt F/B+Pt F/B: patient activation associated with baseline knowledge in cancer history (lower activation with lower score, $\mathrm{p}=0.05$ )

- Model+OC: SCP/PCP decreased worry while SCP only increased worry $(p=0.05)$

- Pt F/B+Pt F/B: high patient activation associated with better baseline care coordination

- Model+Pt F/B: Confidence in survivorship improved from baseline to $\mathrm{f} / \mathrm{u}$ in all survivors, not different by model 
- Model+Pt F/B+OC: no association in level of satisfaction, model, and pt activation

- Model+Prov F/B: no association in PCP confidence and model

- Model+Res+Pt F/B: Survivors reported less responsibility of the oncologist to follow them for cancer at $\mathrm{f} / \mathrm{u}$ and increased discussions about who would provide followup for medical problems other than cancer (oncologist, $82 \%$; PCP, 32\%). Survivors had greater expectations that the PCP would provide preventive health compared to the oncologist and treat other medical problems. Survivors placed similar responsibility on both the PCP and oncologist in screening for other cancers

$\underline{\text { Taylor 20195 }}^{55}$ : Pragmatic phase II pilot RCT evaluating nurse-led survivorship model of care in 60 adult lymphoma survivors from single tertiary cancer center in Australia

- Model: nurse-led lymphoma survivorship model comprised of $3 \mathrm{~F} 2 \mathrm{~F}$ appts, delivery of tailored resources (see Excel abstraction for full list), and SCP shared with PCP; coinciding with heme appt to decrease travel burden

- Pt Char+Pt needs: Women had more unmet needs than men in general; men had higher information needs

- Pt Char+Pt F/B: Women had more anxiety/depression/stress than men

- Model+Pt F/B: control had higher fighting spirit over time compared to model

- Model+Pt F/B: those in intervention had greater confidence in PCP at study completion

- Models+Pt F/B: no association between intervention (survivorship clinic) and anxiety/depression/stress

- Pt F/B: $50 \%$ identified fear of recurrence, $1 / 3$ identified fatigue and/or cognitive impairment

- Model+Res+Pt Use: $83 \%$ recorded goal to increase or start exercise, $53 \%$ wanted to make lifestyle changes, $13 \%$ used the motivational chart to assist with smoking cessation, $10 \%$ quit smoking by study completion, $6 \%$ used motivational chart to address excessive alcohol intake (3\% abstinent, $3 \%$ reduced intake by end of study)

- Res+Prov Pract: 16 (89\%) PCPs had read the SCP and seen their patients within the last six months; 11 (61\%) indicated they had discussed SCP with patient; 16 PCPs completed the Likert-type scale on the usefulness of SCP; majority ( $\mathrm{n}=13,81 \%)$ to SCP was good to very good (M=4.19).Ten (56\%) GPs provided responses which included a desire for more information on treatment, chemotherapy agents, frequency of hematologist review, side effect management and what was required of the PCP

$\underline{\text { Parker 2018 }}^{56}$ : 4-site cluster randomized trial examining efficacy of survivorship planning consultation (communication skills training for physician then applied in survivorship visit with SCP) in 42 physicians of 198 adult lymphoma survivors at the time of remission

- Res: 5 hours of in person communication skills training, including info about lymphoma survivorship, videos of communication strategies for survivorship consultation, standardized pts, and discussion about benefits/barriers to SCP implementation (control arm was 2 hour in person training focused on wellness/lifestyle factors only)

- Model+Res: 15 minute one time new survivorship consult

- Model+Res+Pt F/B: Pts knowledge increased with time in both groups but more for intervention 
- Model+Pt Use: pts in intervention $>2 x$ likely to get flu shot, more likely to get colorectal cancer screening but not pneumococcal shot, other cancer screenings (mammogram, pap smear, skin check), annual PCP visit (overall adherence to preventive care was low)

- Pt F/B+Pt Use: patient knowledge of lymphoma not associated with adherence

Jefford 2016 ${ }^{57}$ : RCT: Survivor Care vs usual care in 221 colorectal cancer survivors in Australia

- Model+Res: Survivor Care: (a) information package, (b) nurse-led, face-to-face end-oftreatment session; (c) a tailored SCP; and (d) telephone followup

- Model+Res+OC: those in SCP group expressed more satisfaction with care; similar levels of psychological distress; cancer survivor needs (measured by Cancer Survivors' Unmet Needs measure (CaSUN)); QoL

\section{Reports From Survivors}

Rosenberg 2017 ${ }^{29}$ : questionnaire in $\mathrm{n}=1713$ adult survivors of adult cancers evaluating a riskadapted visit for developing an SCP (model and resource are intertwined)

- Models/Res+Prov F/B: 95.7 \% (1545) felt that the survivorship nurse was knowledgeable about the information discussed during the RAV

- Models/Res+Pt F/B+Prov F/B: felt more comfortable recognizing signs and symptoms of recurrence to report to their health care provider; $98 \%$ (1583) had a better appreciation for potentially helpful community programs geared to survivorship services

- Models/Res+Pt F/B+Prov F/B: $94 \%$ (1518) felt more confident in their ability to communicate information about their cancer treatments to other members of their health care team; $72 \%$ stated that they had discussed their SCP with their primary care physician (PCP) or another health care provider

- Models/Res+Pt F/B: Survivors were asked to select any of five ways they found the SCP personally useful since the time of their RAV. Of the 488 respondents, nearly $100 \%$ found the SCP a useful tool to summarize medical information, $97 \%$ to reinforce followup care, $85 \%$ to recognize symptoms of recurrence to report, $93 \%$ to identify healthy lifestyle practices, and $91 \%$ to assist in identifying resources for support. Almost $85 \%$ found the SCP a useful tool in all five domains.

- Models/Res+Pt Use: $97 \%$ stated they made at least one positive lifestyle change since their RAV (dietary modification and/or increased physical activity and/or discontinuation of non-prescribed supplements); $89 \%$ attended at least one LIFE health promotion seminar and identified which seminar was attended

- (M) being nurse-led may be the (M) giving the model/resource gas; takes place in 3 location affiliates with one academic center (C); Patients discussed SCP with their PCPs and this could be the catalyst for transitioning back to primary care $(\mathrm{C} / \mathrm{M})$. [author speculation on context-mechanism-outcome hypothesis]

\section{Reports From Providers}

Forsythe 2013 ${ }^{58}$ : Survey of Physician Attitudes Regarding the Care of Cancer Survivors (SPARCCS) survey of oncologists $(\mathrm{N}=1130)$ and PCPs $(\mathrm{N}=1020)$ caring for breast and colorectal cancer survivors

- Res+Prov F/B: Nearly half of oncologists (49.1\%) reported always/almost always providing treatment summaries (TSs), whereas $34.2 \%$ of PCPs reported always/almost always receiving TSs $(\mathrm{P}<.001)$; Furthermore, $20.2 \%$ of oncologists reported 
always/almost always providing SCPs, whereas $13.4 \%$ of PCPs reported always/almost always receiving SCPs $(\mathrm{P}=.001)$.

- Res+Prov Char+Prov F/B+Model: Detailed training in late and long-term effects, use of partial or full electronic medical records in clinical practice, Asian or "other" race/ethnicity, and older age were positively associated with always/almost always providing TSs to PCPs $(\mathrm{P}<.05)$. Detailed training in late and long-term effects, use of partial or full electronic medical records in clinical practice, and Asian race/ ethnicity were positively associated with always/almost always providing SCPs to PCPs, whereas an oncology practice size of 6 or more physicians was inversely associated with SCP provision $(\mathrm{P}<.05)$.

- Res+Prov F/B+Prov Pract: Compared to PCPs who received neither TSs nor SCPs, PCPs who reported always/almost always receiving SCPs were more likely to report never having uncertainty or difficulties related to care coordination, always communicating with other physicians about cancer care and care for other medical issues, and being very confident in knowledge about surveillance, late and long-term effects, and psychosocial needs (all $\mathrm{P}<.05$ ). Moreover, PCPs who reported always/almost always receiving SCPs were more likely than PCPs who received TSs alone to report always communicating with other physicians about cancer care and other medical issues, and being very confident in knowledge about late and long term effects $(\mathrm{P}<.05)$. PCPs receiving TSs alone were more likely than PCPs who received neither document to report never having difficulties transferring patient care responsibilities $(\mathrm{P}<.05)$

- Model+Res: No associations with preferred model of survivorship care or confidence in SCP

- $\operatorname{Res}+\operatorname{Prov}$ F/B: This is a communication issue at its core: a PCP who does NOT receive an SCP reported difficulty transferring patient care responsibilities.

- Model+Res+Prov F/B: PCPs not receiving SCP may not work in the same shared care model as the Onco ( $\mathrm{C}$ and $\mathrm{M})$. [author speculation on context-mechanism-outcome hypothesis]

- $\quad \operatorname{Res}+\operatorname{Prov}$ F/B: Treatment summaries and SCP being combined may support communication and coordination $(\mathrm{C}$ and $\mathrm{M})$. [author speculation on context-mechanismoutcome hypothesis]

- $\operatorname{Res}+\operatorname{Prov}$ F/B: Electronic records may enable implementation of SCPs (M). [author speculation on context-mechanism-outcome hypothesis]

Jabson 2015 $^{\text {59: }}$ : Test associations between receipt of followup care instructions (FCI) and treatment summaries (TS) paired with patient navigation $(\mathrm{PN})$ and survivor's receipt of cancer surveillance, preventive cancer screening, and attendance at regular medical appointments in 3541 cancer survivors who participated in the 2010 LIVESTRONG online survey and "who had completed their cancer treatment"

- Res: "The purposes of patient navigation and SCP are complementary. Patient navigation is designed to improve cancer-related outcomes by reducing barriers to survivor's timely progression through the cancer care continuum, and SCPs are designed to improve health and followup care by informing and directing followup care after cancer treatment is completed"

- Model+Res+Pt Use: Each of the support services was independently associated with survivor's attendance at medical visits, receipt of surveillance for previous cancer, and 
being up to date on preventive care screening. Followup care instructions had strongest association with receipt of followup care. Likelihood of receiving followup care (i.e., attendance at medical appointments and receipt of preventive cancer screening) was greatest when survivors received FCI, TS, and patient navigation.

- Model+Res+Pt Use: In order for SCPs to achieve their maximum intended effect, it is possible that they could be enhanced by patient navigation. If forced to choose only one component of the SCP or patient navigation, the findings suggest that followup care instructions are the best single support service for ensuring followup cancer surveillance. [author speculation on context-mechanism-outcome hypothesis]

\section{Other Study Design}

$\underline{T h o m ~ 2019}^{60}$ : record review of 622 adult survivors of early stage breast, prostate and colorectal cancer from one hospital's (MSK) survivorship clinic

- Models: NP-led survivorship clinic with SCP as part of the intervention

- Models+Prov Char: "NPs were able to deliver care in adherence with established guidance and to function as independent providers"

- Models+Prov Char+Prov F/B: Authors discuss the fact that in NY, where the research was conducted, NPs can bill for their independent visits, which provides a financial structure that supports this model - at least in this policy context. [author speculation on context-mechanism-outcome hypothesis]

\section{Survivorship Care Plans}

\section{Randomized Controlled Trials or Related}

Nicolaije 2015 ${ }^{\mathbf{6 1}}$ : ROGY cluster RCT 12 -month findings in endometrial cancer survivors; $\mathrm{n}=221$ at baseline; $\mathrm{n}=158$ at 6 months, $\mathrm{n}=147$ at 12 months

- $\operatorname{Res}+\mathbf{P t}$ F/B: Intention-to-treat analyses at 12 months showed that patients in the SCP care arm reported receiving more information about other services than patients in the usual care arm. Per-protocol analyses revealed three overall effects: SCP patients reported receiving more information about treatment, other services, and different places of care vs. usual care. Patients receiving SCP care reported receiving more information about things you can do to help yourself get well at diagnosis, treatment at 12 months, and other services at 12 months.

- Res+OC: No overall effects regarding satisfaction but lower satisfaction with nurse interpersonal skills at 12 months in SCP arm in ITT but not per-protocol analysis.

- $\operatorname{Res}+\mathbf{P t}$ F/B: In intention-to-treat analyses, overall, patients in the SCP care arm reported that they experienced more symptoms, were more concerned about their illness, and were more affected emotionally. Per-protocol analyses showed two overall effects: Patients receiving SCP care reported that they experienced more symptoms and were more concerned about their illness. Furthermore, at diagnosis, patients receiving SCP care reported that they experienced more symptoms, were more concerned about their illness, and were more affected emotionally. At 12 months, patients receiving SCP care reported that they were more concerned about their illness and more affected emotionally. 
- Res+Pt Use: Overall, patients receiving SCP care reported having had more cancerrelated contact with their primary care physician than patients in the usual care arm, both in intention-to-treat and per-protocol analyses.

- Res+Pt F/B+OC: SCP care increased the amount of information received, suggesting that SCPs may be useful for providing more patient tailored information in areas for which existing information provision is insufficient. However, SCP care did not show an effect on satisfaction with information provision and care. Future research is needed to investigate whether certain patients may or may not benefit from SCPs. [author speculation on context-mechanism-outcome hypothesis]

- Res+Pt F/B+Prov Pract+OC: Patients in the SCP care arm reported being more concerned about their illness, feeling more affected emotionally, and experiencing more symptoms. However, it is unclear whether these effects are harmful or perhaps beneficial for patients. Health care providers are often reluctant to provide information on potential late effects, because they believe it may have negative effects on psychological adjustment and increase the experience of late effects. However, one could also argue that receiving an SCP raises patients' awareness of cancer related symptoms and empowers them to find the necessary support. The current outcome that patients receiving SCP care reported more cancer-related contact with their primary care physician is in line with this assumption. Because followup care for cancer survivors is increasingly transferred to the primary care physician, SCPs may be a useful tool to enable this transition. [author speculation on context-mechanism-outcome hypothesis]

$\underline{\text { Jeppesen 2018 }}^{\mathbf{6}}$ : ROGY RCT to assess the impact of survivorship care plan (SCP) provision and moderating factors on health care use following endometrial cancer treatment $(\mathrm{n}=221)$

- Res: web-based registration of medical history, treatment, followup care, ROGY enables automatic generation of a survivorship care plan including information of possible late effects, effects on social/sexual life, recurrence signs, psychosocial/supportive care services

- Res+Pt Use: In the first year after diagnosis, women allocated to the SCP group had more visits to their GP compared to women receiving usual care. This difference was only significant directly after treatment (mean 2.7 visits vs. 1.7 visits, $95 \%$ CI: $0.4-1.9$ ).

- Res+Pt Use: At 24-month followup, observed a higher use of GP in the usual care arm (mean 1.2 visits vs. 0.3 visits, $95 \%$ CI: $-1.8-0.2$ )

- Res+Pt Char+Pt Use: Women treated with radiotherapy, and women with anxious symptoms had a higher use of primary care when receiving an SCP compared to those not receiving an $\mathrm{SCP}$

- Res+Pt Use: No difference was found with regard to the cancer-related use of specialist care between the two groups

- Res+Pt Use: The survivorship care plan may help women seek relevant care in the early stages of treatment, but maybe not in later stages. [author speculation on contextmechanism-outcome hypothesis]

deRooii 2017 $^{62}$ : ROGY cluster RCT 24-month findings in ovarian cancer survivors; $\mathrm{n}=174$ eligible (61=SCP, $113=$ control), $n=123$ at 6 -month, $n=100$ at 12 -month, $n=75$ at 24 -month

- Res+OC: No overall differences in satisfaction in the overall ITT analysis. At 6 and 24 months, patients in the SCP care arm reported lower perceived information on other 
services. In the PP analysis ( $\mathrm{N}=153)$, this difference remained only significant after 24 months. Further, in ITT analysis only after 24 months, patients in the SCP care arm reported lower satisfaction with the interpersonal skills of the nurses, but this difference was not significant in the PP analysis.

- Res+Pt F/B: In the ITT analysis overall, after diagnosis and after 6 and 12 months, patients in the SCP care arm reported lower beliefs that the treatment would help to cure the illness compared to patients in the usual care arm. In PP analysis this finding was only significant overall and after 12 months.

- Res+Pt Use: In ITT analysis, patients in the 'SCP care' arm reported less visits to the medical specialist in the 6 months after treatment.

- ovarian cancer patients who received an SCP had less trust that the treatment would help to cure the disease. This may be explained by the fact that ovarian cancer generally has a poor prognosis, and information provided in the SCP (i.e., on chance of recurrence) may not support the patients' belief that she will be cured. This is negative yet realistic information that the patient otherwise would mostly not receive, as oncology providers are often reluctant to provide such information in order to prevent the patient from negative psychosocial effects. [author speculation on context-mechanism-outcome hypothesis]

Nicolaije 2014 ${ }^{63}$ : pre-post intervention questionnaire to assess oncologists $(n=38$ preintervention [21 SCP, 17 control]; $n=35$ [20 SCP; 15 control] post-intervention) perceptions of SCPs used in ROGY cluster RCT of endometrial and ovarian cancer survivors in the Netherlands

- Res+Prov F/B: Both before and after patient inclusions, oncology providers were motivated to start or keep using the SCP $(\mathrm{M}=7.7$ and 7.9, respectively, with $1=$ not at all$10=$ very much; $\mathrm{p}=0.55)$.

- Res+Prov F/B: Before patient inclusion, $61 \%$ of the oncology providers in the SCP care arm expected to need more time for their consultations, with an average of $7.5 \mathrm{~min}$ (range, 2-10 min). After patient inclusion, 25\% reported that they had actually needed more time for their consultations, with an average of $7.3 \mathrm{~min}$ (range, 2-10 min). Selfreported consultation time also did not differ before $(\mathrm{M}=21.8 \mathrm{~min})$ and after $(\mathrm{M}=18.7$ $\min$ ) patient inclusion ( $\mathrm{p}=0.22)$.

- $\operatorname{Res}+\operatorname{Prov} \mathbf{F} / \mathbf{B}+\mathbf{P t} \mathbf{F} / \mathbf{B}$ : When asked to what extent they expected the SCP to affect the patient positively, oncology providers in the SCP care arm reported a 7.3 (with $1=$ not at all-10=very much). After patient inclusion, when asked whether they believed the SCP had actually affected the patient positively, oncology providers reported a $7.5(\mathrm{p}=0.85)$.

- Res+Prov Pract: The oncology providers rated the extent to which the SCP contributed positively to the communication between themselves and the patient, and between themselves and the primary care physician with a 5.4 and a 3.0, respectively (with $1=$ not at all $-10=$ very much).

- Res+Prov F/B: In general, oncology providers rated their satisfaction with the use of the SCP with a 7.1 (with $1=$ not at all-10=very much).

- Res+Prov F/B: Approximately two thirds (64\%) of the oncology providers indicated that they encountered practical problems in the use of the SCP. Most frequently reported practical problems were not finding an appropriate moment to discuss the SCP with the patient (56\%), not completing ROGY in time for the SCP (44\%), and technical issues with ROGY (11\%). Other practical problems that were reported were all related to the 
availability of time to prepare and discuss the SCP. Suggestions for improvement of the SCP were the participation of an oncology nurse in small hospitals to ease the burden on gynecologists, to involve other specialists in the different aspects of the SCP, and to already provide an SCP before treatment.

- Res+Prov F/B: Pragmatic concerns about the implementation of SCPs that were identified in previous studies were finding the time, reimbursement, personnel, and resources necessary to create SCPs [4, 10-14]. In order to overcome these barriers, the circumstances to provide an SCP in the ROGY Care trial were designed to ease the burden on oncology providers as much as possible. By automatically abstracting all the relevant information for the SCP from the registration system ROGY, oncology providers were able to generate an SCP by merely pushing a button. Consequently, in the current study, reimbursement issues and resources necessary to create SCPs were not reported as barriers by the oncology providers. [author speculation on context-mechanism-outcome hypothesis]

- $\operatorname{Res}+\operatorname{Prov}$ F/B: The most frequently reported practical barrier was finding the time to discuss the SCP. Interestingly, the self-reported consultation time of the oncology providers in the SCP care arm did not differ before and after patient inclusions. Moreover, self-reported consultation time did not differ between the SCP care arm and the usual care arm. These findings indicate that there is a difference between oncology providers' perceptions of how much time they spent on SCP care and actual reported consultation time. [author speculation on context-mechanism-outcome hypothesis]

- Res+Prov Pract: oncology providers reported to have low confidence that the SCP contributed positively to the communication between themselves and primary care physicians. For future research, it would be interesting to examine how the contribution of SCPs to the communication between medical specialists and primary care physicians can be increased. Furthermore, primary care physicians' evaluation of receiving an SCP is needed. It is important to note, however, that the SCP in the present study was not specifically tailored to the needs of primary care physicians. [author speculation on context-mechanism-outcome hypothesis]

deRooij 2019 $^{\mathbf{6 4}}$ : analysis of ROGY cluster RCT examining whether the impact of SCPs on PROs differed between patients with an information-seeking coping style (monitoring) versus those with an information-avoiding coping style (blunting); endometrial ( $n=131)$, ovarian $(n=95)$

- Res+Pt F/B: Among patients who had a monitoring coping style, compared with patients in the usual care arm, those in the SCP care arm reported higher receipt of information about treatments, information about things to do to get well, satisfaction with information received, perceived helpfulness of the information received, and higher general satisfaction with care.

- Res+Pt F/B: Among patients who had a blunting coping style, compared with patients in the usual care arm, those in the SCP care arm reported a higher impact of the disease on life and more concerns about the illness.

- Res+Pt F/B: Patients in the SCP care arm with a monitoring coping style who did not use the internet for medical information reported higher helpfulness of the information received, whereas those with a monitoring coping style who did use the internet for medical information did not report higher helpfulness. 
- $\operatorname{Res}+\mathbf{P t}$ F/B: SCPs may be beneficial for patients who desire information about their disease, whereas SCPs may be less beneficial for patients who avoid medical information, suggesting a need for tailored SCP delivery to improve survivorship care. [author speculation on context-mechanism-outcome hypothesis]

- $\operatorname{Res}+P t$ F/B: Monitors more often used the internet to look up information about their disease compared with blunters, whereas SCPs appeared most helpful for monitors who did not do so. These findings suggest that SCPs are most valuable for patients who desire information about their disease but do not have access to resources like the internet. [author speculation on context-mechanism-outcome hypothesis]

- Res+Pt F/B: Determining whether SCPs should be individualized according to information dose only (ie, extensive vs brief SCPs) or also on content (ie, focus on physical vs psychological aspects) 40 will require further research. [author speculation on context-mechanism-outcome hypothesis]

$\underline{\text { Brothers 2013 }}^{\mathbf{6 5}}$ : RCT to examine the impact of receiving a SCP on patients' perceptions of quality of care in physicians who had completed gynecologic oncology fellowship training among gynecologic cancer survivors who were up to one-year post-treatment

- Models+Pt Use: No significant difference in perception of health services and quality of care between patients receiving SCP vs patients who did not receive SCP, for all domains (administrative services, clinical services, educational services, helpfulness of writing materials, overall experience)

$\underline{\text { Majhail 2019 }}^{66}$ : RCT ( $\mathrm{n}=231$ intervention, $\mathrm{n}=227$ control) adult survivors of adult cancers receiving stem cell transplant

- Res+OC: decrease in distress in the intervention group

- Pt Char+OC: lower distress among older patients.

- Res+Pt F/B+Prov F/B: The SCP helped survivors better communicate about HCT and its side effects with their medical providers. The 6-month interview included an openended question about patients' experience with the SCP; dominant themes identified on qualitative analyses included patients reporting that the SCP helped survivors focus on their overall health, supported them in making care decisions with providers, and supported emotional health and coping

- (M) by increasing autonomy patients felt more comfortable making decisions and discussing them with their physicians. [author speculation on context-mechanismoutcome hypothesis]

$\underline{\text { Smith 2016 }}^{\mathbf{6 7}}$ : Randomized controlled trial with qualitative component comparing providerinitiated vs. patient-initiated survivorship care plan; breast, prostate, colorectal cancer patients $(\mathrm{n}=41$ randomized; 25 completed followup [13 provider-initiated, 12 patient-initiated; 13/40 providers invited participated])

- Res: Provider initiated web-based care plan: "Care Plan Builder" vs. Patient initiated web-based care plan: "My Care Plan"

- $\operatorname{Res}+\mathbf{P t} \mathbf{F} / \mathbf{B}$ : patients in the provider-initiated arm had statistically significant improvement on family/partner needing information (mean change: $0.5 ; \mathrm{P}=.04$ ), handling the topic of cancer in social/work situations (mean change: $0.8 ; \mathrm{P}=.03$ ), and exploring spiritual beliefs (mean change: $0.6 ; \mathrm{P}=.04$ ) 
- Res+Pt F/B: In patient-initiated arm, needing help managing my concerns about cancer improved by 0.3 in the provider-initiated arm but worsened by 0.7 in the patient-initiated $\operatorname{arm}(\mathrm{P}=.03)$.

- Res+Prov F/B: limited participation of eligible oncologists in this research initiative (33\%) suggests that the imminent Commission on Cancer accreditation standards do not provide a sufficient incentive for many oncologists to develop systems for survivorship care planning, nor does the Web-based nature or potential patient-initiated structure overcome existing barriers. [author speculation on context-mechanism-outcome hypothesis]

- Res+Pt F/B: Patients showed higher knowledge and confidence when participating in a SCP. [author speculation on context-mechanism-outcome hypothesis]

$\underline{\text { Boekhout 2015 }}^{38}$ : Randomized controlled trial ( $\mathrm{n}=408$ patients [ 337 in analysis] from 9 Canadian cancer centers) 24-month outcomes of health service use and QoL effects of SCP intervention to transition breast cancer patients from oncology to primary care

- Res: Randomized to PCP-led followup with or without SCP. Intervention group had 30minute survivorship visit and SCP. Document and followup visit reminder table sent to PCP.

- Env+Pt Use: Geographic region ( $<<0.01)$, associated with guideline adherence

- Pt Char+OC: SF-36 associated with guideline adherence as was time from completion of primary treatment $(\mathrm{p}<0.013)$

- Res+Pt Use: No difference in receipt of guideline recommended mammography. No difference in post discharge cancer center visits, care coordination

- Res+Pt F/B: SCP not associated with identifying PCP as responsible for follow up. Not associated with cancer-specific distress, psychological distress, Health-related QoL, patient satisfaction

- Res+Model: Less than 3\% of patients visited oncologist for routine follow up once transitioned to a PCP - patient and PCP were willing to have PCP led follow up.

$\underline{\text { Irene Su 2019 }}^{68}$ : Randomized controlled trial comparing online SCP to curated online resource list in young breast cancer survivors ( $\mathrm{n}=182$ : 86 intervention, 96 control), with at least one significant women's health issue

- Res: web-based SCP with information regarding women's health issues+twice weekly text message women's health action prompts compared to control (curated web-based resource lists and twice weekly study adherence text messages)

- Res+OC: positive impact on fertility-related concerns; non-significant impacts on hot flashes, contraception, and vaginal symptoms

- Res+OC: Sixty-one intervention group participants (70.9\%) improved, compared to 55 controls $(57.3 \%)(\mathrm{OR} 1.82,95 \% \mathrm{CI} 0.99-3.4, \mathrm{p}=0.057)$ on at least one issue (primary outcome).

$\underline{\text { Hershman 2013 }}^{\text {69: }}$ : Single-blinded randomized trial evaluating in-person SCP with 126 breast cancer survivors (60 control, 66 intervention). Secondary objective to look for differences between Hispanic and non-Hispanic patients (41\% Hispanic. Hispanic were less educated, less likely to be married, less likely to be F/T employed, lower income, less likely drinker) 
- Res: All participants given NCI "Facing forward: Life after cancer treatment". Intervention group also met with NP and nutritionist for an hour to receive personalized summary and recommendations

- Res+Pt F/B: SCP associated with less health worry

- Res+Pt F/B: No association at 3 months with SCP and cancer worry, or function or depression

- Res+OC: Although the negative outlook scale was higher in control group at 3 months, the change from baseline wasn't significant between groups. At 3 months the health worry subscale was higher for control group, but this did not persist at 6 months.

- Res+Pat Char: No interaction between treatment and ethnicity

Teverwaark 2019 ${ }^{70}$ : RCT in which breast cancer patients $(\mathrm{n}=127)$ were randomized to two arms: (1) immediate receipt of an individualized SCP and (2) delayed receipt of an individualized SCP (i.e., 4 weeks after baseline). Conducted between November 2013 and December 2014.

- Res+Pt F/B: Positive increase (4\%) in individual knowledge between baseline and week 12 for both arms; receipt of SCP does not appear to be driving the improvement (at week 4 , there was no difference in knowledge between those who received the SCP at baseline, and those who were about to receive the SCP at week 4;

- Res+Pt F/B+OC: No change in satisfaction with communication and care coordination over time

- Res+Pt F/B: At baseline, the population was generally well-informed (76/8\% of answers were correct). The current "1-size-fits-all" approach to SCP provision may not benefit all survivors (those who are already well informed or for whom reviewing the information is particularly distressing). [author speculation on context-mechanism-outcome hypothesis]

- Res+Pt F/B: Authors hypothesized that the improvement in knowledge was because of repeated administration of the knowledge survey. "We suspect that much of the improvement was the result of survivors seeking out information in response to their own uncertainty with regard to the correct survey answers." Further research into the best strategies for improving survivor knowledge is warranted (e.g., small, targeted pushes of key information, "test-your-knowledge" surveys, and/or reviews in video or group sessions). [author speculation on context-mechanism-outcome hypothesis]

- Res+OC: "We hypothesize that satisfaction declined as each survivor's attention was repeatedly drawn to knowledge gaps" suggesting a potentially harmful consequence of SCP provision." [author speculation on context-mechanism-outcome hypothesis]

- (CY): Note mediating effect of patient's baseline knowledge on the effect of resources (like SCPs); goes with the theme of varying impact of information. [author speculation on context-mechanism-outcome hypothesis]

Maly 2017 ${ }^{71}$ : RCT: usual care vs. individualized SCP provided with nurse counseling in 212 lower income Latina breast cancer survivors

- Res+Prov Pract: increased physician implementation of recommended care with SCP and counseling (mean score 60.8 vs 48.6 , scores 0 to 100)

- Res+Pt Use: patient adherence to recommended care increased, but not statistically significantly (score 55.7 vs 48.7 )

- $\operatorname{Res}+\mathbf{P t}$ F/B: no impact on 'perceived efficacy in patient-physician interactions' 
- Res+Prov Pract: They argue first RCT to show clinical outcome improvement with SCP (outcome being PCP implementation of rec care). [author speculation on contextmechanism-outcome hypothesis]

$\underline{\text { Fang 2020 }}^{\text {72}}$ : RCT of web-based survivorship care plan app vs usual care in 165 breast cancer survivors within 5 yrs of tx completion, from single medical center in Taiwan

- Res+Pt F/B: decrease in unmet needs with SCP app after 6 months (specified significance in domains of physical and psychological effect, information, and communication); no difference between groups in symptom distress, anxiety, depression

- Res+Pt F/B: decrease in fear of recurrence for both groups but significantly more with SCP app after 12 months

- Res+OC: increase in QoL with SCP app compared to control after 12 months (trend in increased QoL over time for both groups)

\section{Prospective Cohort Studies}

$\underline{\text { Hua 2020}}^{\text {73: }}$ : Single-center intervention study at a community oncology practice in the Midwest to evaluate the impact of delivery SCPs by examining the awareness of SCP receipt as well as how provision affects survivors' perception of care quality and of their condition; $\mathrm{N}=30$ ( 24 in final analysis) cancer survivors (breast, colon, and prostate)

- Res: Most participants preferred to review the SCP with a cancer navigator (58\%), as part of a regular followup visit (63\%), via a paper document at the end of the visit (66\%).

- Res+Pt F/B: 34\% of survivors could not correctly identify whether they received an SCP (suggesting that communication about the SCP may need to be clearer); Increase in (1) survivor activation and involvement in care, and (2) perceived knowledge of care was significant at 4-week followup.

- Res+OC: Satisfaction with knowledge did not change significantly at followup.

- Res: Future research should delve into the reasons why not all survivors who receive SCPs are aware that they have. "As it is evident that not everyone who receives an SCP is aware they have, providers should be explicit during followup visits about the information that survivors are receiving, and where they can go to access it so they may utilize it optimally." [author speculation on context-mechanism-outcome hypothesis]

Chrischillies 2015 $^{74}$ : Prospective cohort to evaluate relationship between SCP and survivorship care and health outcomes reported by 832 long-term lung and colorectal cancer survivors

- Pt Use+Res: $25 \%$ reported receiving a written summary of their treatment and instructions on who to see for routine care; $47 \%$ indicated they received either a written summary of their treatment or instructions on who to see for routine care (but not both); and $28 \%$ received neither SCP element

- Pt Char+Res: Older people and lung cancer survivors were significantly less likely to report receiving survivorship care planning, while those who received chemotherapy were more likely.

- Res+OC: Physical health $(\mathrm{p}=0.012)$ and good-to-excellent self-perceived health status (OR 2.2; $95 \%$ CI 1.3-3.9) were better for those receiving both elements

- Res+Pt Use: Participants receiving both SCP elements were more certain which doctor was in charge, more likely to report followup checkup, and had an MRI/PET/CT scan in the past 2 years compared to those receiving neither. 
Palmer 2015 $^{75}$ : Longitudinal, quasi-experimental, pre-post pilot study to explore outcomes associated with delivery of an SCP in $n=139$ stage $0-3$ breast cancer survivors from 7 NCIdesignated cancer centers

- Res+Pt F/B+Pt Char: individuals closer to diagnosis reporting greater use of SCP

- Res+OC: Satisfaction with SCPs was significantly positively related to time since diagnosis

- Res+OC: Approximately 56\% reported being "very" or "extremely satisfied" with SCP, whereas only $10 \%$ of participants reported being "unsatisfied."

- $\quad \operatorname{Res}+\mathbf{P t}$ F/B: Overall, participants reported significant increases in perceived coordination among providers

- $\operatorname{Res}+\mathbf{P t}$ F/B: knowledge concerning cancer care increased significantly after the SCP visit

- $\quad$ Res+Pt F/B+Pt Char: Change in perceived knowledge was also independent of time since diagnosis. Perceived provider knowledge increased after the SCP visit, independent of time since diagnosis. Increased coordination was independent of time since diagnosis.

- time since diagnosis (C.M) impacted some of the outcomes-- may infer more time for pt to use the SCP and gain more benefits (M)

$\underline{\text { Nissen 2013 }}^{\text {76}}$ : survey pre and post receipt of treatment summaries (17-month interval) in 344 breast and colorectal cancer survivors who requested the summaries

- Res+Pt F/B: improved Pt knowledge of nodal status, morphology and receptor status of breast cancer; did not improve knowledge of treatment received (but this was already very high, $>96 \%$ accurate pre-treatment summary); year of diagnosis knowledge declined

- Res+Pt Char: breast cancer survivors and younger more likely to use treatment summaries but all mostly relied on their own memory

- study rationale based on our phrase 'can share their history, know risks' etc. through receipt of treatment summaries. i.e., without universal health record patients need to convey to clinicians diagnosis, treatment, etc. [author speculation on context-mechanismoutcome hypothesis]

$\underline{\text { Mayer 2014 }}^{77}$ : Prospective, descriptive single arm study looking at feasibility, usability and satisfaction with SCP for colon cancer survivors; 34 SCPs, 28 stage 1-3 colon cancer survivors finishing treatment, 15 PCPs

- Models+Res: oncology nurse completed Journey Forward SCP and delivered to patient by oncology NP during routine f/u visit, SCP mailed to PCP

- Res+Prov F/B: 49 minutes to complete surgery SCP, 90 minutes surgery+chemo SCP -$>$ too labor intensive

- $\operatorname{Res}+\operatorname{Prov}$ F/B: 16 minutes to deliver surgery SCP, 26 minutes to deliver surgery+chemo SCP - NPs didn't feel SCP added to time of visit, but provided structure

- $\operatorname{Res}+$ Pt F/B: survivors identified before treatment ended or within first 3 months was preferred time to get SCP

- $\operatorname{Res}+$ Pt F/B+OC: survivors found SCP easy to use/understand, high satisfaction and usability ratings 
- $\operatorname{Res}+$ Prov F/B: PCPs rated SCP usability highly but commented it was too long for their use

$\underline{\text { Napoles 2019 }}^{\mathbf{7 8}}$ : Longitudinal cohort study to evaluate the feasibility, acceptability, and preliminary efficacy of a culturally and linguistically suitable SCP for Spanish-speaking breast cancer patients $(n=23)$ in public hospital settings, approaching the end of active treatment

- Res: Nuevo Amanecer (New Dawn) Survivorship Care Planning Program: smart phone app for Spanish speaking cancer patients; weekly calls with health coach; adapted from the American Clinical Society of Oncology (ASCO) template for low-literacy, Spanishspeaking Latinas

- Res+ Pt F/B+Pt Use+OC (Positive): Compared with baseline, postintervention fatigue and health distress levels were significantly lower and knowledge of recommended followup care and resources and emotional well-being improved significantly; selfefficacy for managing cancer followup care did not change

- Pt Char+Pt F/B: Tailoring programs for language and culture could make long term management more feasible for minority populations. [author speculation on contextmechanism-outcome hypothesis]

$\underline{\text { Brant 2016 }}^{\text {79: }}$ : Prospective cohort intervention at The Billings Clinic, an integrated cancer center in Montana, to examine symptom and quality-of-life (QOL) trajectories survivors and to explore patient, caregiver, and PCP satisfaction with receipt of a SCP. $\mathrm{N}=67$ patients with breast cancer or lymphoma, $\mathrm{N}=39$ caregivers, $\mathrm{N}=23$ PCPs

- Res: Survivorship care plan (SCP) delivered by a survivorship navigator (who conducted the survivorship visit, followed up with the PCP regarding the SCP, and answered phone calls from patients enrolled in the study about persistent symptoms and survivorship concerns)

- Res+Pt Needs: Patients with more comorbidities and higher symptom burden were more likely to telephone the survivorship navigator.

- Res+OC: Majority of patients $(n=49)$ strongly agreed that they were satisfied with the SCP.

- $\quad \operatorname{Res}+$ Pt F/B: At first, most patients perceived that their PCP was better able to manage their needs with the SCP, but this declined at six months

- Res+Pt F/B: Authors argue that the SCP may help patients feel more confident about their PCP's ability to provide survivorship care HOWEVER, they note that this perception declined at 6 months. (CY: May echo findings from Tevaarwerk, 2019 that SCPs may highlight knowledge gaps by individual and/or primary care team). [author speculation on context-mechanism-outcome hypothesis]

$\underline{\text { Hebert 2017 }}^{\mathbf{8 0}}$ : Pre-experimental research design with a non-equivalent control group, an end of treatment (T0), and a three-month followup (T1) to assess and document the feasibility and acceptability of implementing an individualized survivorship care plan among women with endometrial cancer ( $n=18$ intervention; $n=12$ control), oncology nurse navigators $(n=2)$, general practitioners $(\mathrm{n}=12)$ in Canada

- $\quad \operatorname{Res}+\mathbf{P t} \mathbf{F} / \mathbf{B}$ : at followup, the group with an ISCP had lower scores on all needs subscales: (1) information: $35 \%$ of the exposed group versus $74 \%$ of the control group, $\mathrm{p}=$ .030 ; (2) professional and financial: $6 \%$ with an ISCP versus $19 \%$ control, $p=.057 ;$ (3) 
access and continuity: $9 \%$ with ISCP versus $25 \%$ control, $\mathrm{p}=.078$; (4) relational (support): $18 \%$ with ISCP versus $50 \%$ control, $\mathrm{p}=.007$, and; emotional: $13 \%$ with ISCP versus $28 \%$ control, $\mathrm{p}=.044$.

- $\operatorname{Res}+\mathbf{F} / \mathbf{B}$ : differences in empowerment not generally different between groups at followup, except ISCP group presents a trend towards a higher score than the control group on the skill and technique acquisition sub-scale 75.00 (10.21) versus 64.06 (10.67), $\mathrm{p}=.097$.

- $\quad$ Res+Pt F/B: No difference in fear of recurrence; both intervention and control groups had clinically significant fear of recurrence

- Res+Prov F/B: a positive impact on needs seems to be observed, although it does not have a repercussion on distress or behaviors of empowerment. [author speculation on context-mechanism-outcome hypothesis]

$\underline{\text { Nahm 2019 }}^{81}$ : (pre-post $\mathrm{n}=30$ adult survivors of adult cancers evaluating interactive electronic Cancer Survivorship Patient Engagement Toolkit (CaS-PET) developed to deliver survivorship care plans

- Res+Pt Use+OC: In adult survivors of cancer, this SCP has a positive impact on diet and exercise, better physical QOL, reduced symptom burden, particularly physical symptoms

- (M) potentially is increasing awareness via the SCP

\section{Reports From Survivors and Providers}

$\underline{\text { Morken 2019 }}^{\mathbf{8 2}}$ : Cross-sectional survey of hematopoietic stem cell transplantation (HSCT) survivors $(n=29)$ and nontransplantation clinicians $(n=18)$ (oncologists and primary care) to determine their HSCT survivorship information needs (SCP content and format, preferred timing of SCP provision)

- Res: sample 17-page HSCT-specific SCP with a treatment summary autopopulated from an EHR. The SCP included a diagnosis and treatment summary, care team contact information, followup care and health maintenance recommendations, information on late and chronic effects of treatment, and HSCT survivor resources.

- Res+Prov F/B+Pt F/B: Content that was perceived to be very useful for the following:

- Clinicians: radiation treatments received, preventative screenings

- Patients: Diagnosis, Cancer workup and staging, contact information for care team, all sections on GVHD

○ Both: recommended vaccinations

- $\operatorname{Res}+\operatorname{Prov}$ F/B+Pt F/B: Much of the highly rated and personalized content is not included in generic SCP templates (e.g., care team contact information, dates/results of most recent laboratory tests); areas of improvement (for the sample SCP): more information about GVHD and immunosuppresion, timeliness for exposures to avoid, increased clarity in survivor and clinician roles, simplification of language, and a desire for the SCP to be dynamic and personalized.

- $\operatorname{Res}+\operatorname{Prov}$ F/B+Pt F/B: Delivery: $93 \%$ of patients preferred a paper SCP at the end of a clinic visit / 89\% of clinicians preferred a copy in the EHR. Both survivor and clinician groups would also accept SCPs as a copy on an electronic patient portal, mailed letter or fax, or a secure PDF via email.

- $\operatorname{Res}+\operatorname{Prov} \mathbf{F} / \mathbf{B}+\mathbf{P t} \mathbf{F} / \mathbf{B}$ : If the SCP is not up-to-date or specific to what the patient is currently experiencing, it will be less useful or not used at all. 
- $\operatorname{Res}+\operatorname{Prov}$ F/B: "We believe using the EHR for creation and provision of HSCT-specific SCPs is the best option. Benefits include reducing the time and resources needed to compile records to create an SCP, creating an electronically searchable document, and allowing for updates to the plan as the survivor progresses through follow-up care." "However, an SCP will be unlikely to optimize survivorship care if (1) the EHR and clinical workflows do not support discrete HSCT data capture, (2) work systems do not have organizational policies and technologies that are designed to support clinician needs, and (3) roles and responsibilities to support clinical workflows for accomplishing survivorship tasks are not clearly assigned. [author speculation on context-mechanismoutcome hypothesis]

Campbell 2012 ${ }^{83}$ : Survey of 20 patients and their physicians ( 21 physicians or dentists) between 3 and 4 years after receipt of the SCP to determine the perceived value of SCPs

- Res+Pt F/B: Of the 20 survivors, only 2 (10\%) remembered receiving the SCPs (when asked 3 years after). Only 1 survivor said the document was useful, had referred to it, and still had it available.

- Res+Prov F/B: Of the 32 community physicians and dentists, 34\% could either locate the SCP document or recalled having received it.

- $\operatorname{Res}+$ Pt F/B: A potential barrier to successful SCP implementation in our population includes the insensitivity of the template SCPs to individual survivors' levels of health literacy. [author speculation on context-mechanism-outcome hypothesis]

- Res+Prov F/B: Another barrier might have been the volume of data generated during cancer care; a single document, even an important summary, becomes a needle in a haystack for other health care professionals who use differing EHRs or paper charts. [author speculation on context-mechanism-outcome hypothesis]

$\underline{\text { Ashing 2013 }}^{84}$ : Get perspective from 12 Latina breast cancer survivors/advocates and 10 health care providers on the Treatment Summary and Survivorship Care Plan (TSSCP-S) Spanish language template

- Res+Pt F/B: TSSCP-S template was rated more favorably than the TSSCP-ASCO on the following domains: content $(\mathrm{p}=0.02)$, clarity $(\mathrm{p}=0.02)$, utility $(\mathrm{p}=0.04)$, cultural and linguistic responsiveness $(p=0.03)$, and socioecological responsiveness $(p=0.01)$.

- $\operatorname{Res}+P t$ F/B: Several evaluators noted that the TSSCP-S template was more patient-userfriendly

- $\quad \operatorname{Res}+$ Pt F/B: Evaluators noted that perhaps some more commonly-used Spanish words might be used to replace some of the ones selected by the template translators. Others noted that there was plenty of medical terminology that makes the form appear as though it is geared towards the medical providers

- Res+Pt Char: Preference of survivors for culture and language specific SCPs, could be better for comprehension, knowledge gain, and follow through. [author speculation on context-mechanism-outcome hypothesis]

$\underline{\text { Benci 2019 }}^{85}$ : Analyzed data from survivors, family members, providers $(n=46,408)$ who completed OncoLink to generate SCP

- Pt Char+Prov Char+Prov F/B+Env most linked to receipt of SCP was cancer type, provider type (those treated by combo of oncologist and PCP most likely to receive SCP), 
receipt of Tx summary, geographic region (NE least likely), setting (those treated at private offices lowest rate of SCP)

\section{Reports From Survivors}

Jabson 2015 $^{\mathbf{8 6}}$ : Subset findings from RefID 3364, which tested associations between receipt of followup care instructions (FCI) and treatment summaries (TS) and survivor's receipt of cancer surveillance, preventive cancer screening, and attendance at regular medical appointment in 3541 cancer survivors who participated in the 2010 LIVESTRONG online survey and "who had completed their cancer treatment"

- Res+Pt Use: Survivors who received FCI were:

- more than two-fold as likely to report attendance at medical visits

- $62 \%$ more likely to report receipt of surveillance

- more than two-fold as likely to report receipt of preventive screenings

- (compared to survivors who did not receive FCI)

- Res+OC: Survivors who received FCI were:

- $25 \%$ fewer late/long term effects

- (compared to survivors who did not receive FCI)

- Res+Pt Use: Survivors who received TC were:

○ $79 \%$ more likely to report attendance at medical visits

- $41 \%$ more likely to report receipt of preventive screenings

- (compared to survivors who did not receive TS)

- TS was not associated with receipt of cancer surveillance or number of late/long term effects

- Res+Pt Use+OC: "the evidence could suggest that when cancer survivors receive FCI, they become more aware of the symptoms for late/long term effects sooner and pursue necessary care earlier, thereby diminishing the number of symptoms reported." Further investigation into the relationship between TS and late/long term effects is needed to clarify the mixed findings and understand the value of TS in survivorship care. [author speculation on context-mechanism-outcome hypothesis]

Jabson 2014 ${ }^{87}$ : To determine how treatment summary (TS) and followup care instructions (FCI) related to cancer survivors' cancer-related pain using in 7917 cancer survivors who participated in the 2010 Behavioral Risk Factor Surveillance System, Cancer Survivorship module (crosssectional survey)

- OC: Pain was reported by $9 \%$ of survivors.

- Res+OC: Compared with cancer survivors who had not received TS or FCI, those who received TS had greater odds of pain (OR, 1.57; 95\% CI, 1.08-2.28) and those who received FCI had more than twice the odds of pain (OR, 2.36; 95\% CI, 1.52-3.67); odds of pain were largest among survivors who had received FCI or TS and had less than a high school education;

- Pt Char+OC: Odds of pain were nine times higher for survivors aged 18-34 years compared with other age groups.

- Res+OC: It is possible that receipt of FCI and TS heightens cancer survivors' awareness and report of cancer-related pain, but they do not diminish the occurrence of cancerrelated pain. Our finding also may be explained by the severity of cancer treatment (those 
who undergo more severe cancer treatments are more likely to receive FCI and TS). [author speculation on context-mechanism-outcome hypothesis]

$\underline{\text { Hawkins-Taylor 2019 }}^{\mathbf{8 8}}$ : Examine the use of and assess patient satisfaction with survivorship care plans among 189 Stage 1-3 adult cancer survivors from 5 treatment centers in 2 health systems in SD

- Res+Pt Use: patients reporting any use of SCP were 11.1 times more likely to report having undertaken a health action. $84 \%$ of survivors reported taking a health action attributed to SCP.

- Res+Pt Char: patients with male reproductive cancer were 4 times less likely to be satisfied with SCP information

- Pt Char+Pt Use: no demographic or cancer-related characteristics were associated with undertaking a health action

- Prov Char+OC: Nurses have an ongoing relationship with patients that is based on trust, therefore providing them with ability to increase patient satisfaction in the use of SCPs. [author speculation on context-mechanism-outcome hypothesis]

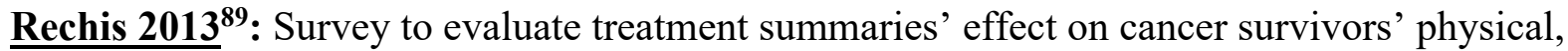
emotional and practical patient concerns through an online survey $(\mathrm{N}>12,000)$

- Res+OC: Participants who did not report receiving a treatment summary experienced more emotional concerns vs those who did receive a treatment summary, $p<0.05$,

- Res+OC: Respondents who did not receive a TS were more likely to report that they had learned to live with their concerns, physical $(51 \%)$ and emotional $(58 \%$; both $\mathrm{P}<.01)$

- Res+Pt Use: Participants who received a TS were more likely to report that their care providers met all of their needs both during and after treatment (both $\mathrm{P}<0.01$ )

$\underline{\text { Blanch Hartigan 2015 }}^{90}$ : Cross-sectional survey to assess the relationship between treatment summary receipt and patient-centered communication (PCC) and overall quality of care in 359 cancer survivors who had received treatment summaries in the Health Information National Trends Survey

- Res+Pt Char: Survivors who had reported two or three or more types of cancer treatments were over two times as likely to have received a treatment summary compared with survivors who only had a single type of cancer treatment $(\mathrm{OR}=2.30, \mathrm{p}=.003$ and $\mathrm{OR}=2.49, \mathrm{p}=.03$, respectively).

- Res+OC: Survivors who received a treatment summary were over 3 times as likely to report excellent/very good care quality compared to those who did not receive a treatment summary $(\mathrm{OR}=3.44, \mathrm{p}=.005)$.

- Res+Pt F/B: Survivors who received a treatment summary reported higher PCC scores compared with survivors who did not receive a treatment summary (unstandardized beta $=7.03, \mathrm{p}=.004)$

- Res+Pt F/B: Receipt of treatment summaries was associated with a higher likelihood of optimal PCC for exchanging information $(\mathrm{OR}=1.93, \mathrm{p}=.024)$, making decisions $(\mathrm{OR}=$ $1.96, \mathrm{p}=.020)$, enabling self-management $(\mathrm{OR}=1.91, \mathrm{p}=.027)$, managing uncertainty $(\mathrm{OR}=1.94, \mathrm{p}=.015)$ and fostering healing relationships $(\mathrm{OR}=2.13, \mathrm{p}=.010)$. 
$\underline{\text { Cahine 2019 }}^{91}$ : population-based survey of 1,514 breast, colorectal, melanoma, other cancer survivors in Canada using modified Cancer Survivors Unmet Needs Measure (CaSUN)

- Pt Char: (Tbl 3): survivors below the age of 34 were more likely to disagree with overall needs met, information availability, support for physical concerns, and practical challenges than those of other age groups; males were more likely to agree with receiving support for physical concerns $(84.8 \%)$ than females $(79.9 \%)$. No differences based on cancer type or comorbidities.

- Res+OC: those who received a SCP reported higher agreement with having needs met in all domains (overall, information, physical, emotional, and practical) than those who did not

$\underline{\text { Rosenberg 2019 }}^{92}$ : survey study; pilot in 60 adult survivors of lymphoma, breast, colorectal testicular or prostate cancer in the survivorship program at Dana-Farber

- Res+participation in research study: SCP can increase uptake of participation in research in survivorship// this study was looking at 'effectiveness' of using survivorship visit to provide survivors with research opportunities; 44\% expressed interest and 6/30 eligible enrolled in study

$\underline{\text { Lyson 2020 }}^{93}$ : Cross-sectional survey to assess the content and communication appropriateness of 16 SCPs collected from breast and colorectal cancer from diverse health care settings (safetynet, community hospitals, academic centers, and integrated delivery systems)

- Res+Prov F/B: The plans ranged from including an average of $48 \%$ of the IOM recommended components (safety-net plans), 51\% integrated delivery, 56\% community hospital, $60 \%$ of the recommended components (academic medical center plans).

- $\operatorname{Res}+\operatorname{Prov} \mathbf{F} / \mathbf{B}+\mathbf{P t} \mathbf{F} / \mathbf{B}$ : None of the SCPs received a "superior" overall suitability rating (SAM) score, which includes readability and cultural appropriateness scores.

- $\operatorname{Res}+\operatorname{Prov} \mathbf{F} / \mathbf{B}+\mathbf{P t} \mathbf{F} / \mathbf{B}$ : By hospital type: academic hospitals had the highest mean SAM rating of $47 \%$ (adequate), community hospitals 39\% (not suitable), safety-net health systems 38\% (not suitable), and IDSs $29 \%$ (not suitable)

- $\operatorname{Res}+\operatorname{Prov}$ F/B+Pt F/B: Only one plan in received a "superior" rating for cultural appropriateness. The remaining plans failed to demonstrate tailoring for diverse racial/ethnic groups and received an "adequate" score for presenting information most appropriate for English-speaking, Caucasian patient populations

$\underline{\text { Rosales 2015 }}^{94}$ : evaluation of pilot process to deliver SCPs to breast cancer patients $(\mathrm{n}=118)$ in a multidisciplinary visit in 5 outpatient clinics in southern Idaho

- $\quad$ Res: SCP completed by Registered Health Information Technologist (RHIT). Interdisciplinary 1-hour survivorship visit jointly run by oncology NP and oncology social worker. Visit reviews SCP, discusses effects of treatment, surveillance and care coordination. Description of sharing document with PCP. Social worker completes psychosocial assessment. Survivorship info and goals provided, and 2 SMART goals are set. SCP sent to referring physician and PCP.

- $\operatorname{Res}+\mathbf{P t}$ F/B+OC: 50 of 118 were contacted in follow up call; 88\% (of 50) understood plan, $86 \%$ said visit met their needs, $80 \%$ working on goals

- $\operatorname{Res}+\operatorname{Prov}$ F/B: $6 \%$ return on investment on average (calculation of cost for time spent vs. reimbursement) 
$\underline{\text { Tarver 2019 }}^{95}$ : Survey (open- and close-ended questions) to characterize the usefulness of a CRC personal health record (PHR) and survivorship care plan and to describe the usability of these technologies in 22 colorectal cancer survivors recruited from academic and VA health systems in Indiana

- Res: Colorectal Cancer Survivor's Personal Health Record (CRCS-PHR): developed by adapting an open-source electronic health record (OpenMRS) [20] to deliver an online survivorship care plan to CRC survivors; Users could create online relationships with their doctors of choice, whether primary care or specialist physicians.

- Res+Pt F/B: CRC survivors perceived features related to their health care (ie, summary of cancer treatment history, followup care schedule, description of side effects, and list of community resources) to be more useful than communication features (ie, creating online relationships with family members or caregivers, communicating with doctor, and secure messages). CRC survivors typically described utilizing traditional channels (eg, via telephone or in person) to communicate with their health care provider. Participants expressed interest in communicating with other CRC survivors in online networks in order to have a support group of individuals who had similar experiences. $77 \%$ reported preferring to have access to the resource "right away" at diagnosis. Major challenges experienced by participants included barriers to system log-in, lack of computer literacy or experience, and difficulty entering their patient information.

- Res+OC: Participants had overall positive perceptions with respect to ease of use and overall satisfaction.

Frick 2017 ${ }^{96}$ : cross sectional survey in 117 pancreatic cancer survivors to evaluate publicly available internet-based SCP tool, only 21 of whom completed 5-question survey about SCP

- Res: OncoLink and OncoLife SCP from Penn; 24\% had received a treatment summary, $5 \%$ survivorship information following treatment for pancreatic cancer; SCP completed by survivor $50 \%$, family/friend in $31 \%, 16 \%$ by HC provider (13 nurses, $6 \mathrm{NPs}$ )

- Models: $47 \%$ had ongoing care from oncologist, 35\% from oncologist+PCP; \% seeing oncologist decreased $\mathrm{w} /$ time $-86 \%$ of survivors were managed or comanaged by an oncologist if less than 2 years from diagnosis vs $72 \%$ if 2 or more years from diagnosis (301 of 462 survivors); increase in PCP alone mgmt (11\% if less than 2 years in followup to $22 \%$ after)

- $\operatorname{Res}+$ Pt F/B:38\% felt amount of info was adequate, $50 \%$ felt there was not enough info

- Res+Pt Use: $83 \%$ reported they would share the plan with their providers

$\underline{\text { Frick 2018 }}^{97}$ : cross sectional survey; to evaluate publicly available internet-based SCP tool in 964 adult lymphoma survivors, only 150 completed 5 question survey regarding SCP

- Res: OncoLink and OncoLife SCP from Penn

- Models: Few survivors (13\% of 964) reported survivorship health services being offered at their treatment center; $13 \%$ and $4 \%$ had received previous survivorship information or a treatment summary, respectively; $49 \%$ had ongoing care from an oncologist or onc +PCPs (19\%); \% seeing oncologist decreased w/ time (97\% of survivors were managed or comanaged by an oncologist if less than 2 years from diagnosis vs $65 \%$ if 2 or more years from diagnosis (301 of 462 survivors)); increase in PCP alone mgmt (4\% if less than 5 years in followup to $43 \%$ once $5+$ years); $50 \%$ (of 150 completing 5 question 
survey of SCP) did not know if their cancer ctr had survivorship program, 15\% said theirs did

- Res+OC: $84 \%$ (of 150 ) rated satisfaction with care plan as good (33\%), v good (32\%), or excellent (19\%). 53\% felt amount of info was adequate, $40 \%$ felt there could be more or there was not enough

- Res+Pt Use: $66 \%$ reported they would share the plan with their providers; $29 \%$ did not feel info was relevant to their current care and $27 \%$ thought their provider would not care

\section{Reports From Providers}

Birken 2017 ${ }^{98}$ : cross sectional survey of 23 cancer programs in South Atlantic Division of American Cancer Society regarding SCP use

- Models+Res: $52 \%$ were community hospital comprehensive cancer programs, $10 \% \mathrm{NCI}$ designated comprehensive cancer programs, $38 \%$ teaching hospital cancer programs; $86 \%$ reported someone had used SCPs at least once; $76 \%$ said $<25 \%$ of providers had ever used an SCP, 100\% reported SCPs used in $<$ half of survivors; $62 \%$ reported SCPs were delivered to half or fewer survivors for whom SCPs were completed, $50 \%$ reported SCPs were delivered to $25 \%$ or fewer PCPs

- Res+Prov F/B:61\% reported the cancer program began using SCPs due to professional societies' recommendations (though $62 \%$ reported a barrier was that some societies do not yet require SCP use for accreditation)

- Res+Prov F/B: barriers to SCP use cited -- 75\% reported insufficient organizational resources including time, staff, money, training; open ended responses included lack of systems to facilitate use such as electronic SCP that autopopulates from EMR, lack of systemic referral to survivorship RN

- Res+Prov F/B: no relationship between SCP use and barriers (e.g., barriers did not differ between programs reporting no vs some SCP use; no assoc between barriers and \% providers using SCPs or \% being delivered to pts)

- Models+Res: no assoc between programs reporting no SCP use and program type (though all programs reporting no SCP use were teaching hospitals)

- Models+Res: no assoc between program type and \%providers using SCPs or $\%$ delivered to pts

Birken 2014 $^{99}$ : cross sectional survey of 81 US cancer programs with membership in at least one cancer care QI organization looking at determinants of SCP use

- Res: $44 \%$ used SCPs (30\% sometimes, $15 \%$ regularly); of 56\% not using SCPs, $93 \%$ planned to in future

- Res: of those using SCPs, $58 \%$ only had $<1 / 4$ providers ever using SCPs; of these, $69 \%$ said SCPs were used for $<50 \%$ of pts; of these, $61-63 \%$ reported SCPs were delivered $<=50 \%$ of pts or PCPs

- Res+Pt Char: current/planned SCP use limited to breast (82\%) and colorectal (56\%)

- Res+Prov F/B: Most common barriers: lack of resources (76\%), difficult to use (29\%), influential ppl didn't advocate for their use (24\%)

- Res+Prov F/B: Reason for use: COC standards (78\%), SCPs may improve qual of care (75\%), NCCN guidelines (54\%) 
- Res+Env: Academic program and those with National Cancer Institute Community Cancer Centers Program membership more likely to indicate current SCP use (both $(89 \%$ vs $38 \%, \mathrm{p}<0.01)$; freestanding programs less likely to use SCP (0 vs $48 \%, \mathrm{p}=0.02)$

- Res+Prov F/B: our results suggest that support, specifically intended to facilitate SCP use, may promote SCP use more effectively than the non-specific resources (e.g., time, staff, training, money) that are commonly cited as determinants of SCP use. [author speculation on context-mechanism-outcome hypothesis]

Birken 2019 $^{\mathbf{1 0 0}}$ : cross sectional survey regarding SCP implementation of 395 cancer care providers (of $69 \mathrm{~K}$ potential) from various cancer groups (e.g., CoC, ONS)

- Res+Pt Char: SCPs primarily used for breast, colorectal, and lung cancers

- Res: $61 \%$ report SCP developed within 3 months of completion of adjuvant tx, similar for delivery to $\mathrm{pt} ; 1 / 3$ delivered via mail, $>50 \%$ in person delivery; SCPs developed within individual programs $(42 \%)$

- Res+Prov Char: 50\% said nurse navigators are responsible for each stage of SCP implementation; onc NP/oncologist develop the f/u part of SCP (22/26\%) and deliver SCPs to survivors $(31 / 24 \%)$

- Res+Prov F/B: $>2$ hours to complete all stages of SCP implementation, not reimbursed except delivery

- Res+Porv F/B: EHR/automated processes were underused for developing SCPs, while direct communication was underused in SCP delivery

Merport 2012 $^{\text {101}}$ : Survey of cancer specialist physicians $(\mathrm{n}=108)$ and PCPs $(\mathrm{n}=400)$ in Massachusetts to estimate the current use of treatment summaries and care plans, receipt of treatment summaries and care plans by PCPs, and obstacles to use among cancer specialist physicians

- Res+Prov Char: 56\%) prepare treatment summaries; $14 \%$ prepare SCPs

- Res+Prov F/B: $47 \%$ of specialists identified having no training as a barrier, $46 \%$ had no template available and $40 \%$ reported no reimbursement for time for preparation

Dittus 2014 ${ }^{102}$ : Survey of 39 primary care providers (PCP) regarding SCPs developed for breast and colorectal cancer survivors in their practice and to determine whether PCP response to the SCPs varied according to characteristics of the practitioner and their practice

- Res: JourneyForward SCP

- Prov Char+Prov F/B+Env: $15 \%$ disagreed that the care plan helped them understand their role in facilitating survivorship care and $15 \%$ disagreed that they understood how responsibility would be shared with the oncologist. The PCPs from the rural site were less likely to agree that the SCP contributed to their understanding of their role (pinteraction $=0.05$ ).

- Prov Char+Prov F/B+Env: the rural PCP's were less likely than the urban PCPs to agree that the SCPs provided sufficient detail about monitoring for late and long term effects and tests to obtain for monitoring. There was no significant difference in the perception of SCP content detail by years in practice

- Res+Prov F/B: Over 70\% of PCP's at each site agreed that SCPs would improve communication with medical providers and patients. 
- Res+Prov F/B: Among those with $\leq 18$ years in practice, $95 \%$ of PCPs agreed that the SCP would improve communication with patients, whereas only $60 \%$ of PCPs with $>18$ years in practice agreed ( $\mathrm{p}$-interaction $=0.03$ ).

- Model+Prov F/B: The most common barrier to providing followup care was "limited access to survivors since they stay with their oncologist" with $64 \%$ considering this a significant or moderate barrier.

- Prov Char+Prov F/B: PCP's practicing for less than 18 years were significantly more likely to feel that insufficient knowledge of cancer survivorship issues was a barrier to survivor care (79\% vs. $36.8 \%)$.

- Res+Prov Char+Env+Prov F/B: There is a difference in perspective between PCPs with different characteristics (this example rural/urban and years of experience). [author speculation on context-mechanism-outcome hypothesis]

$\underline{\text { Salz 2012 }}^{103}$ : Cross-sectional survey to determine 156 PCPs' preferences for the content of survivorship care plans for colorectal cancer (CRC) survivors

- Prov Char+Prov F/B: The topics which respondents needed more information were: knowing the reason for terminating chemotherapy $(60 \%)$, whether there were any problems with chemotherapy (67\%) or radiation therapy $(60 \%)$, and whether complementary services were provided during treatment (psychosocial $(66 \%)$, nutritional (66\%), and other (63\%) supportive services).

- Prov Char+Prov Pract: 70\% felt they did not have enough information on whether the oncologist intended to monitor the patient for cancers at other sites.

- Prov Char+Prov F/B: A substantial minority of PCPs reported not having enough information about key clinical aspects of a cancer diagnosis, such as stage (40\%) and grade (44\%); F/B: $83 \%$ wanted more information about genetic counseling and testing to identify high-risk individuals; $78 \%$ typically needed more information about increased risks for second colorectal cancers, other cancers, and other diseases.

- Prov Char+Prov F/B+Res: Nearly all respondents would like to receive a summary of the diagnosis $(100 \%)$, a summary of treatment $(99 \%)$, recommendations for ongoing care the patient should receive from the PCP (96\%), and information from the patient's cancer care provider about what aspects of care after treatment the PCP and the cancer care provider are each responsible for $(97 \%)$.

$\underline{\text { Donohue 2019 }}^{104}$ : Cross-sectional survey (November 2016) of primary care physicians and advanced practice providers (APPs) with the objectives of (1) understanding the real-world utilization of SCPs within a primary care outpatient context and (2) assessing the impact that SCPs had on clinical decision-making within primary care clinic visits for breast cancer survivors. $\mathrm{N}=40$ PCPs and APPs participated $(\mathrm{n}=40 / 88,45 \%$ response rate) within a large academic center utilizing a single EHR

- Res: Survivorship care plan (SCP) [generated through the EHR, survivor-facing]

- Models+Res: Most primary care visits (60\%) included discussion of cancer or cancerrelated issues. However, providers largely did NOT use the SCP for the visit (only 10\% (4/40) reported using the SCP for the visit in question). Instead, most cancer-related information was obtained by asking the patient or family (79\%) or checking oncologist 
notes in the EHR (75\%). One-third of providers aware of the SCP reported using it ( $\mathrm{n}=$ $4 / 11)$

- Res+Prov F/B: Barriers to SCP use: being unaware of the SCP (73\%), difficulty locating it $(30 \%)$, and finding needed information faster via another mechanism $(15 \%)$

- Res+Prov F/B: "There appears to be a persistent and significant barrier to SCP utilization at the point-of-care despite increasing SCP provision as well as primary care education." Even though the SCPs have been housed in a standardized location (the EHR problem list), primary care utilization of SCPs has not substantially increased within our system. We speculate that perhaps we have not reached a "critical mass" of routine provision to enough survivors over a sufficient duration. However, additional significant barriers to SCP utilization may very well lie within the realm of integration into primary practice. We should address the assumption that primary care providers know where to locate SCPs and can readily integrate them into routine clinical practice. Future work needs to address primary care-centered design of SCP format and content, location in the EHR, and the ability to "push" relevant or needed survivorship information to primary care at the right time. [author speculation on context-mechanism-outcome hypothesis]

- relates to use of information. [author speculation on context-mechanism-outcome hypothesis]

Donohue 2015 $^{105}$ : Examine perspectives regarding EHR-generated SCPs for breast cancer survivors among 81 PCPs who had or were currently seeing survivors enrolled in one of University of Wisconsin's survivorship clinical trials

- Res+Prov F/B: Main barriers to SCP use were not knowing a plan existed (83\%), not knowing how to find the plan $(75 \%)$, not being able to locate the plan in the patient chart (71\%), and not knowing to look for the plan (72\%); potential facilitators to SCP use were increased awareness of plan existence (85\%), standardized location within medical records $(89 \%)$, and consistent provision of care plans for all patients (81\%); many PCPs (57\%) indicated that a PCP-facing SCP (rather than an SCP designed to be both PCP and patient facing) would facilitate use; most PCPs (89\%) preferred to receive plans via the EHR

- Res+Prov F/B: Our data suggests that PCPs may prefer a shorter plan (1 - 3 pages), oriented to clinicians, with a uniform location in the medical record). Future research into clinician-oriented care plans; challenges of consistently providing, delivering, and updating care plans; and developing processes for better informing PCPs regarding the existence and value of care plans. [author speculation on context-mechanism-outcome hypothesis]

$\underline{\text { Ashing 2016 }}^{\text {106}}$ : Survey to evaluate a Treatment Summary and Survivorship Care Plan for African-American breast cancer (TSSCP-AABC) in 54 healthcare professionals from researchbased medical centers, community oncology and general medical practices, nurses associations, and survivor advocates

- Res: The TSSCP-AABC was rated more favorably than the standard TSSCP-ASCO by health professionals on all domains: content $(\mathrm{p}<0.01)$, clarity $(\mathrm{p}<0.01)$, utility $(\mathrm{p}<0.01)$, cultural responsiveness $(p<0.01)$, socioecological responsiveness $(p<0.01)$

- Res+Prov Char: PCPs rated clarity of the TSSCP-AABC more highly than the oncology health professionals $(\mathrm{p}<0.01)$. "May be influenced by oncology professionals' extensive 
experience with the complexities of cancer, from patients' perspectives, and practical understanding that documentation is not equivalent to patient education".

- Res+Pt Char: Targeted SCP for specific groups can be considered more favorable for patient centered care. [author speculation on context-mechanism-outcome hypothesis]

\section{Other Study Designs}

$\underline{\text { Hamlish 2020 }}^{\mathbf{1 0 7}}$ : Retrospective review of 109 breast cancer survivors' charts at five federally qualified health centers (FQHCs) in Chicago (35 MDs, 9 APNs, and 6 PAs) to understand breast cancer care coordination at FQHCs before implementation of COC treatment summary and survivorship care planning standard, looking at how external information facilitates cancer care coordination for underserved populations

- Prov F/B+Prov Pract: The presence of external cancer records did not increase documentation of PCP engagement in breast cancer-relevant followup care. Neither external records nor PCP documentation of tumor information (i.e., breast cancer pathology, lymph node status, or hormone receptor status) correlated with care engagement. The most significant predictor of PCP care engagement was when PCPs ascertained information about their patient's breast cancer treatments themselves. Greater PCP documentation of their patient's breast cancer treatment correlated with higher rates of cancer-relevant followup.

- Prov F/B+Prov Pract+Res: This paper suggests that an important mechanism for increasing PCP engagement is the act of PCPs' eliciting information for himself/herself (e.g., through patient history taking, talking to the cancer specialist directly). On the one hand, this could represent a form of sensemaking / processing of information. On the other hand, it could just be a way to avoid sifting through the mess of information in the EMR - in which case - a resource such as the TS-SCP would be helpful. [author speculation on context-mechanism-outcome hypothesis]

- Prov F/B+Prov Pract+Res: PCPs more frequently incorporate information into patient care when they elicit it themselves / may be due to information in the EMR being "unorganized and difficult to find." "As a result, providers find themselves navigating a data storm." The TS-SCP attempts to address effective communication; however, "our work suggests providing information is not enough." (CY: I don't fully agree with this interpretation - seems like having a tool such as the TS-SCP that might help navigate the "data storm" would be beneficial). [author speculation on context-mechanism-outcome hypothesis]

- ROGY trial looks at information from the patient perspective; this study provides insights regarding information from the provider perspective. [author speculation on contextmechanism-outcome hypothesis]

$\underline{\text { Philips 2020 }}^{108}$ : Retrospective evaluation comparing knowledge of cancer history and health maintenance recommendations among survivors who had received an $\mathrm{SCP}(\mathrm{n}=80)$ vs. those who hadn't $(n=126)$ from a community cancer center in US southwest

- Res+Pt Char: Receipt of SCP was associated with current working status (those working more likely to have had an SCP) $(\mathrm{p}<0.05)$

- $\operatorname{Res}+$ Pt F/B: Patients who received SCP were less likely to correctly answer what screening tests for other cancers were recommended (p.0.001). Breast patients who 
received chemo were more likely to know when this ended if they had had an SCP $(\mathrm{p}<0.05)$.

- Res+Pt Char: remission, health insurance, working at diagnosis, health insurance, working during treatment were NOT associated with receipt of SCP

- Res+Pt Char: In analysis only of breast patients, SCP receipt was not associated with knowledge of stage.

\section{Workshops}

Chen 2015 ${ }^{\mathbf{1 0 9}}$ : Exploratory, secondary analyses of data from 127 cancer survivors participating in the "Cancer: Thriving and Surviving" online self-management program

- Res: 6-week workshop containing both educational material on cancer survivorship skills and a social networking aspect. A self-management program for cancer survivors named "Cancer: Thriving and Surviving" (developed by Stanford and the University of Hawaii Cancer Center). Based on self-efficacy theory and techniques to enhance efficacy (e.g., weekly action plans, modeling by peer facilitators, and discussions among participants, reinterpretation of symptoms by describing them as having many causes, and social persuasion by having public disclosure of both action plans and action plan completion).

- Res+OC: Writing posts on the online discussion board was associated with improvements in health outcomes, but reading posts was less important. Completing, but not merely making, action plans and self-tailoring were statistically associated with future positive health outcomes.

- Res+OC: "We often control participants interactions by asking questions or completing set exercises. It would appear that self expression of support is an important component of this and possibly other interventions." Those who posted the most appeared to get the most benefit, indicating that helping others (posting) is in and of itself an important intervention. Allowing participants to freely choose (i.e., self-tailoring) both the problem they wish to work on and the way(s) in which they will address this problem may have positive results. / "The common denominator was that the program provided an efficacyenhancing structure in which these individuals could gain confidence in their abilities." [author speculation on context-mechanism-outcome hypothesis]

Risendal 2014 ${ }^{110}$ : Survey of adults and adult caregivers $(\mathrm{n}=191)$ who participated in the Cancer Thriving and Surviving (CTS) workshop to explore factors in the implementation of an adapted chronic self-management program

- Res+OC: satisfaction with content, leaders, and group process were all high, with 65$81 \%$ indicating they were very satisfied and an additional $16-24 \%$ indicating they were somewhat satisfied. Less than $5 \%$ of people indicated they were only a little satisfied; The majority of participants indicated that the content on coping and decision-making was appropriate in length ( $80-90 \%$, respectively). In contrast, $25 \%$ of participants indicated the section on CAM was too short.

Sabatino 2013 ${ }^{111}$ : 2010 National Health Interview Survey including adult survivors (of adult cancer) not in treatment $(\mathrm{N}=1345)$ diagnosed in the last 4 years

- Res+Pt Char: age $(p=0.0035)$, race $(p=0.0173)$, education $(p=0028)$, income $(p=0.0007)$, insurance $(\mathrm{p}=0.01)$, diagnosis $(\mathrm{p}=0.0046)$, treatment modality $(\mathrm{p}=0.0064)$, treatment recency $(\mathrm{p}=0.0033)$, clinical trial participation $(\mathrm{p}=0.0001)$ are significantly associated 
with reporting receipt of followup instructions for all survivors; diagnosis less than 4years NS. for all participants, years since diagnosis greater than 4 years was sig associated with receipt of followup $\mathrm{p}=0.0001$

- Res+Pt Char: Adjusted analyses: Race is significantly associated with reporting receipt of TS for all survivors, $\mathrm{p}=0.0001$. Most recent diagnosis is associate with receiving a TS, $p=0.0024$ ( $p=0.0256$ for $p t$ diagnosed in the last 4 years). Years since diagnosis $(>4$ years $)$ is associated with receipt of treatment summary $(p=0.018)$ and followup plan $(p<$ $0.0001)$. Treatment summaries are more likely reported received by those diagnosed within 4 years based on treatment modality $(\mathrm{p}=0.0296)$, as are receipt of followup instructions for all time since diagnosis $(\mathrm{p}=0.0116)$. Clinical trial participants are significantly more likely to report receipt of treatment summaries and followup instructions. Patients with excellent to very good health status are also significantly more likely to report receiving TS and followup instructions.

- Pt F/B: many survivors are unsure who manages their followup, are insufficiently aware of their continuing health risks, and may lack regular followup for possible late effects and recommended services.(M). [author speculation on context-mechanism-outcome hypothesis]

- Res+Pt Char: Greater reported receipt of treatment summaries by colorectal vs. breast cancer survivors both overall and among those recently diagnosed is somewhat surprising given the relatively early promotion of breast cancer care plan templates. Potential contributing factors might include differences in treatment modalities, facilities, or providers. Breast cancer survivors were more likely to report receiving radiation (44 vs. $19 \%$ ), hormonal treatments (26 vs. $<1 \%$ ), and chemotherapy (39 vs. $33 \%$ ). (M). [author speculation on context-mechanism-outcome hypothesis]

- Res+Prov Pract: written followup instructions may be more highly associated with some recommended surveillance than screening, most notably provider surveillance recommendations. $(\mathrm{C} / \mathrm{M})$ [author speculation on context-mechanism-outcome hypothesis]

$\underline{\text { Shay 2019 }}^{112}$ : Survey of off-treatment adult survivors of adult cancer $(n=1855)$ in BRFSS

- Res+Pt Char: Overall, 37\% (669/1855) of survivors reported receiving a written survivorship care plan. In bivariate analyses, receipt of a SCP was associated with higher levels of educational attainment, being currently uninsured, cancer type (i.e., having a cervical cancer diagnosis), current provider type (i.e., seeing an oncologist or surgeon for majority of care), exercise in the past month, and up-to-date mammography $(\mathrm{p}<0.05)$

- Res+Pt Use: Logistic regression model found positive associations between receipt of a SCP and recent medical appointments, exercise, non-smoking status, and mammography but no association with colorectal cancer screening

- Pt Char+Pt Use: Racial differences were found in several health behaviors, with black survivors being significantly more likely to have attended a recent medical checkup, but less likely to have exercised in the past month, or to report having a mammography in the past 2 years. Additionally, Hispanic survivors were less likely to be up-to-date on colorectal cancer screening.

- Res+Pt Use: Additionally, our secondary analysis does not allow us to explore the mechanisms by which receipt of a survivorship care plan impacts health outcomes. It may be that the act of planning itself motivates survivors to adhere to recommended health 
behaviors, or it may be that the actual written document acts as a reminder and guidepost for followup care. Additionally, the cross-sectional nature of BRFSS data does not allow for test of causality. [author speculation on context-mechanism-outcome hypothesis]

Ostroff 2004 ${ }^{113}$ : Two-phase study to examine interest in and barriers to participation in a multiple family group intervention (MFG) for adult head-and-neck cancer survivors and their family caregivers recruited from Memorial Sloan-Kettering registry. In the first phase, subjects completed a survey addressing quality of life issues $(\mathrm{n}=80)$. In the second phase, patients and caregivers were invited to participate in a one-day MFG workshop designed to address posttreatment psychosocial adjustment issues

- Res: Multiple family group intervention (MFG) was developed to assist families in coping with the persistent challenges of cancer diagnosis, treatment, and rehabilitation. MFG was designed as a 6-hour day-long "workshop" cofacilitated by two mental health clinicians

- Res+Pt F/B: Only 15 of the 80 [19\%] agreed to attend the MFG workshop (even after they made every attempt to accommodate scheduling needs) ; not able to identify any psychosocial predictors of MFG participation.

- Res+OC: Those families who did participate in the MFG workshops were quite positive in their endorsement of the experience and urged us to make it more widely available to other families

- Res+Pt Char: Patients who refused to participate in the MFG groups were more likely to be older, suggesting the family focus of this program was perceived to be more germane to patients/caregivers at an earlier phase in the family life cycle. Given the need to enhance the reach and acceptability of promising MFG interventions, further research exploring patients' and families' reluctance or reasons for declining MFG participation is warranted. [author speculation on context-mechanism-outcome hypothesis]

\section{Other Resources}

\section{Prospective Studies}

Chubak 2014 $^{114}$ : Survey to describe perspectives on survivorship care one year after cancer diagnosis from patients with breast, colorectal, and lung cancer enrolled in a randomized controlled trial (RCT) of a nurse navigator intervention to improve support, communication and coordination of care around the time of diagnosis and through treatment, $\mathrm{N}=230$ in an integrated care delivery system

- Model: The majority considered their cancer specialist (such as medical, radiation, surgical or gynecological oncologist) to be their main provider for cancer followup

- Model+Pt F/B: Participants who considered their PCP to be their ideal provider of cancer followup care were more likely to report confidence in the PCP's skills, knowledge, and communication

- Model+Pt F/B: Approximately half of patients were uncertain how well their PCP communicated with the oncologist and how knowledgeable s/he was in caring for cancer survivors, with breast cancer patients expressing greater uncertainty 
- Model+Pt F/B: patients who indicated their PCP was their ideal doctor for cancer-related followup were more likely to report discussions with their oncologist about who would provide their non-cancer care

- Pt Char+Pt F/B: None of our results in any of the analyses differed meaningfully when restricted to patients with at least 6 months between their last treatment and their survey

Wiljer 2010 ${ }^{115}$ : pilot prospective study of survivorship consult's impact on self-efficacy in 40 breast cancer survivors

- Res: 1-hour reflective interview (survivorship consultation)

- Res+Pt F/B: info very useful -- 75\% dx/tx, 77\% support systems, $59 \%$ challenges in various domains, $80 \%$ care plan recommendations; very likely to pursue more info - $80 \%$ for $\mathrm{dx} / \mathrm{tx}, 64 \%$ support systems; $2 / 3$ report info on putting plans in place to solve identified challenges very useful, $61 \%$ very likely to put plans into place; best time for interview $-35 \%$ at $\mathrm{dx}, 30 \%$ during tx, $20 \%>6$ months post tx, $12.5 \% \mathrm{w} / \mathrm{i}$ first 6 months post tx

- Res+F/B: non-significant increase in Stanford self-efficacy score (7.5 to 7.8), significant increase in Cancer Behavior Inventory median score (median change 101.5 to 103.0) pre/post intervention

Turner 2019116: 3-arm RCT comparing Head and Neck Cancer Survivor Self-Management Care Plan (HNCP) delivered by nurses $(\mathrm{n}=10)$ for patients who had completed treatment for head and neck cancer $(\mathrm{n}=36)$ vs. survivorship information $(\mathrm{n}=36)$ vs. usual care $(\mathrm{n}=37)$.

- Res: HNCP consisted of one face-to-face hour-long meeting in which the patient's treatment was recorded, as were contact details of health professionals involved in their care and followup schedules. Patients were guided to nominate up to three goals for their future well-being and assisted to devise an action plan to achieve these. The HNCP was given to the patient and a copy was forwarded to their PCP. HNCP was based on principles of chronic disease self-management and survivorship care plans advocated by the Institute of Medicine with a focus on promotion of self-efficacy of patients to manage their own health concerns.

- Res+OC: For the primary outcome at 6 months, the intervention group did not differ significantly from the usual care group on any QoL measure; the only significant mean difference in quality of life was for the information group on the physical well-being domain (mean different $0.4, \mathrm{p}<0.05$ ). For secondary outcomes, for the intervention group, at 6 months (relative to baseline), the magnitude of change was significant for (1) maintaining activity and independence, (2) accepting the cancer, (3) seeking social support.

- Res+Pt F/B: Interviews with 14 participants who had received the intervention revealed that although most recalled the nurse-delivered interview, only one had actually used the HNCP. None had discussed the HNCP with their PCP as instructed. Five participants indicated that a single meeting was insufficient for their needs and more intensive support and followup were required.

- Res+OC: The reason for improvement in the physical well-being of those who received information only is not clear. It may be that these patients felt isolated (as expressed by interview participants) and, in the absence of any specific guidance, focused on the written resource which included information about physical activity and specific 
exercises to improve range of movement. The failure of our HNCP to improve quality of life may be due to the timing or insufficient "dose" of the intervention. Our results indicate that more active steps are needed to facilitate PCP-patient followup rather than reliance on a posted written care plan. [author speculation on context-mechanismoutcome hypothesis]

Cairo 2020 $^{117}$ : Non-randomized controlled trial to examine if use of a $\$ 65$ per month app in 127 Stage 1-3 breast cancer survivors

- Res: \$65/month app (VIDA) that gives breast cancer survivors access to a health and wellness coach, maintaining regular video/phone consultation and motivation vs. selfguided toolkit and one-time health education session

- $\quad$ Res+Pt Use: More patients in the VIDA app group experienced weight loss and greater reduction in BMI $(\mathrm{p}<0.01)$, improvement in strenuous physical activity $(\mathrm{p}=0.04)$, and improvement in dietary patterns $(\mathrm{p}<0.001)$; non-significant reduction in fatigue and improvement in depression

- Res+Pt F/B: At 12 months none of the participants were using the app anymore due to cost and technical difficulties

\section{Surveys}

Cheung 2010 $^{118}$ : Cross-sectional survey of 431 cancer survivors to explore the associations among cancer survivorship discussions, patient-physician expectations, and receipt of followup care in cancer survivors

- Prov Pract+Pt F/B: A conversation about cancer followup was associated with a statistically significant higher mean concordance score between patients and oncologists, but not for patients and their PCPs or between physicians.

- Prov Pract+Pt F/B: Survivorship care expectations were most discrepant between PCPs and oncologists (mean score, 1.78), moderate between patients and oncologists (mean score, 1.97), and most similar between patients and PCPs (mean score, 2.82).

$\underline{\text { Belkora 2006 }}^{119}$ : cross sectional survey of the satisfaction with a visit preparation resource from 67 cancer survivors (not childhood) from 3 rural, underserved resource centers in Northern California

- Res: Consultation plan document -- a structured interview with pt that helps pts make a list of questions/set an agenda for an upcoming office visit

- Res+OC: concordance of breast cancer provider and breast cancer pt increased satisfaction

- Other provider characteristics, patient characteristics, and resource center site were not associated with satisfaction

$\underline{\text { Bazzell 2015 }}^{\mathbf{1 2 0}^{20}}$ : Cross-sectional capstone project that assessed unmet needs in 52 rural cancer survivors and developed a pamphlet to educate about available evidence-based interventions or resources and promote use of a shared care model

- Res: Intervention included identifying survivorship resources in the target rural area and linking survivors with these resources using a pamphlet (but this pamphlet was not evaluated) 
- Env+Pt Char+Pt F/B: association between unmet needs related to access and continuity of care and year(s) since diagnosis in a rural population (directionality not clear)

- Env+Pt Char+Pt F/B: association between age and unmet emotional needs (directionality not clear)

- Env+Res+F/B: Access to interventions and survivorship resources were found to be limited in these rural areas. Interventions or resources found to exist require technology access or substantial travel

- Env+Pt F/B: Providers must understand that a considerable amount of the resources presently available to rural survivors are technology dependent. As such, assisting survivors may involve technology instruction as well as taking steps to make technology available. Unfortunately, the vast majority of face-to-face EBP interventions were found to be located in more urban areas and substantial travel by the survivors is required.

Playdon 2016 $^{121}$ : Survey of the Study of Cancer Survivors-I (survivors of 10 most common cancers) to investigate information needs and preferred resources (educational material, supportive tools and services) for long-term cancer survivors

- Pt F/B: Need for more information on cancer screening tests for other cancer types (42.5\% participants), more information on long term cancer side effects $(33.1 \%$ participants), more information on healthy lifestyle behaviors (31.9\%), and more information on tests post cancer treatment (27.0\% participants)

- Pt F/B: Need more information health insurance/finance consequences of cancer $(28.2 \%$ participants)

- Res: Preferred source of information reading a book, magazine, or other printed publication (60.9\% participants), followed by personalized reading materials $(57.6 \%$ participants), and meeting in person with a health care professional (44.4\% participants)

- Pt Char+Pt Needs+Pt F/B: Younger patients, patients with more education, patients with fair/poor self-reported health, and patients of race/ethnicity other than non-Hispanic white had more information needs.

\section{Not Specific to a Model or Resource}

$\underline{\text { deRooij 2018 }}^{\mathbf{1 2 2}^{2}}$ : cross-sectional self-administered survey of 292 early-stage cancer survivors (predominantly if not completely adult cancers) to characterize patterns of health care needs

- describes needs classifications and how sociodemographics are associated with them, but no connection with models or resources. [author speculation on context-mechanismoutcome hypothesis]

- Pt Needs: the highest unmet needs were related to the domains of side effects (53\%), self-care (51\%), and emotional coping (43\%).

- Pt Char+Pt Needs+Pt F/B: Our analysis identified four clusters of survivors: (a) low needs $(\mathrm{n}=123,42 \%)$, (b) mainly physical needs $(\mathrm{n}=46,16 \%)$, (c) mainly psychological needs $(\mathrm{n}=57,20 \%)$, and $(\mathrm{d})$ both physical and psychological needs $(\mathrm{n}=66,23 \%)$. Compared with cluster 1 , those in clusters 2,3 , and 4 were younger $(\mathrm{p}<.03)$, those in clusters 3 and 4 had higher levels of psychological distress $(p<.05)$, and those in clusters 2 and 4 reported higher levels of fatigue $(p<.05)$.

- The identification of these subgroups and sociodemographic and clinical predictors of each subgroup does not diminish the need to screen each individual patient for 
survivorship care needs. It does, however, provide clinical insight into how programs to address the needs of diverse survivors might be structured, and it underscores the importance of both tailored information provision and accounting for differences in needs when testing interventions in survivorship care research. [author speculation on contextmechanism-outcome hypothesis]

- our study may provide an explanation for the failure of some survivorship interventions, such as SCPs, to improve outcomes in randomized trials [9-12]. The effect of these interventions may be diluted by a substantial subgroup of survivors with few or no needs. [author speculation on context-mechanism-outcome hypothesis]

Brennan 2016 ${ }^{123}$ : cross sectional study of 68 Australian early stage breast cancer survivors' needs and satisfaction with care soon after completing treatment

- Pt Char+OC: younger women $<=51$ (compared to older women 52-68 and $>68$ ) reported significantly lower QoL in all areas (physical, emotional, functional) except social wellbeing

- Pt Char+Pt F/B: younger women $<=51$ (compared to older women 52-68 and >68) reported higher number of unmet needs overall and higher number of mod and weak strength unmet needs

- Pt Char+Pt F/B: no assoc between age and satisfaction with overall care and care coordination

- Most common unmet need: fear of cancer recurrence, then expectations/image as cancer survivor

- High level of satisfaction with overall care $(9.2 / 10)$ and care coordination $(8.6 / 10)$

Ford 2019 ${ }^{124}$ : cross sectional survey on factors influencing adherence to CRC surveillance from 150 stage 1-3 colorectal cancer survivors identified from S Carolina Central Cancer Registry Prov Pract+Pt Use: doctor telling them regular f/u care was needed more likely to be adherent to office visit and CEA tests

- Res: $75 \%$ sought cancer info from one or more sources (internet, friends, support group)

- Res+Pt Use: $44 \%$ never received written summary of cancer care, $98 \%$ still reported good to excellent quality of care since treatment

- Pt Use: adherence $55 \%$ cscope, $92 \%$ office visit, $73 \%$ CEA

- Model: $86 \%$ saw PCP, $83 \%$ surgeon, $71 \%$ med onc, $62 \%$ GI since ending treatment; $53 \%$ reported cancer care decisions made with equal input from family and doctor, $30 \%$ with input from family while considering doctor's input

$\underline{\operatorname{Lim}}_{2013}{ }^{125}$ : cross-sectional survey of survivors recruited from the California Cancer Surveillance Program and hospitals in LA (1) to compare family communication, supporting the patient in making decisions, patient's confidence level in communicating with physicians, and health-related quality of life (HRQOL) between Chinese- $(\mathrm{n}=86)$ and Korean- $(\mathrm{n}=71)$ American breast cancer survivors (BCS), and (2) to investigate how family communication, decision support, and self-efficacy in patient-physician communication influences HRQOL for Chineseand Korean-American BCS.

- Pt F/B+OC: For Chinese Americans: direct, positive association between self-efficacy in patient-physician communication and HRQOL 
- Pt F/B+OC: For Korean Americans: direct, positive association between family communication and HRQOL

- Pt F/B+OC: Hypothesis of a direct relationship between decision support and HRQOL was not supported

- Pt F/B+OC: Socio-cultural differences between two ethnic subgroups might influence how communication components among survivors, family, and healthcare providers are associated with HRQOL. 


\section{References}

1. Klosky JL, Cash DK, Buscemi J, et al. Factors influencing long-term follow-up clinic attendance among survivors of childhood cancer. Journal of cancer survivorship : research and practice. 2008 Dec;2(4):225-32. doi: 10.1007/s11764-008-0063-0. PMID: 18787958.

2. Blaauwbroek R, Tuinier W, Meyboom-de Jong B, et al. Shared care by paediatric oncologists and family doctors for long-term follow-up of adult childhood cancer survivors: a pilot study. The Lancet Oncology. 2008 Mar;9(3):232-8. doi: 10.1016/s1470-2045(08)70034-2. PMID: 18282804 .

3. Casillas J, Oeffinger KC, Hudson MM, et al. Identifying Predictors of Longitudinal Decline in the Level of Medical Care Received by Adult Survivors of Childhood Cancer: A Report from the Childhood Cancer Survivor Study. Health services research. 2015 Aug;50(4):1021-42. doi: 10.1111/1475-6773.12282. PMID: 25600956.

4. Christen S, Vetsch J, Mader L, et al. Preferences for the organization of long-term follow-up in adolescent and young adult cancer survivors. Supportive care in cancer : official journal of the Multinational Association of Supportive Care in Cancer. 2016 Aug;24(8):3425-36. doi: 10.1007/s00520-016-3157-7. PMID: 26988228.

5. Svedberg P, Einberg EL, Wärnestål P, et al. Support from healthcare services during transition to adulthood - Experiences of young adult survivors of pediatric cancer. European journal of oncology nursing : the official journal of European Oncology Nursing Society. 2016 Apr;21:105-12. doi: 10.1016/j.ejon.2016.02.008. PMID: 26952685.

6. Casillas J, Syrjala KL, Ganz PA, et al. How confident are young adult cancer survivors in managing their survivorship care? A report from the LIVESTRONG ${ }^{\text {TM }}$ Survivorship Center of Excellence Network. Journal of Cancer Survivorship. 2011;5(4):371-81. doi: 10.1007/s11764-011-0199-1. PMID: 104603085. Language: English. Entry Date: 20120330. Revision Date: 20171109. Publication Type: journal article.

7. Absolom K, Greenfield D, Ross R, et al. Predictors of clinic satisfaction among adult survivors of childhood cancer. European journal of cancer (Oxford, England : 1990). 2006 Jul;42(10):1421-7. doi: 10.1016/j.ejca.2006.01.053. PMID: 16759851.
8. Landier W, Chen Y, Namdar G, et al. Impact of Tailored Education on Awareness of Personal Risk for Therapy-Related Complications Among Childhood Cancer Survivors. Journal of clinical oncology : official journal of the American Society of Clinical Oncology. 2015 Nov 20;33(33):3887-93. doi: 10.1200/jco.2015.62.7562. PMID: 26324371.

9. Signorelli C, Wakefield C, McLoone JK, et al. Childhood cancer survivorship: barriers and preferences. BMJ supportive \& palliative care. 2019 Nov 11. doi: 10.1136/bmjspcare-2019002001. PMID: 31712388.

10. Signorelli C, Wakefield CE, Johnston KA, et al. Re-Engage: A Novel Nurse-Led Program for Survivors of Childhood Cancer Who Are Disengaged From Cancer-Related Care. Journal of the National Comprehensive Cancer Network : JNCCN. 2020 Aug;18(8):1067-74. doi: 10.6004/jnccn.2020.7552. PMID: 32755982.

11. Szalda D, Pierce L, Hobbie W, et al. Engagement and experience with cancer-related follow-up care among young adult survivors of childhood cancer after transfer to adult care. Journal of cancer survivorship : research and practice. 2016 Apr;10(2):342-50. doi: 10.1007/s11764-0150480-9. PMID: 26303367.

12. Suh E, Daugherty CK, Wroblewski K, et al. General internists' preferences and knowledge about the care of adult survivors of childhood cancer: a cross-sectional survey. Annals of internal medicine. 2014 Jan 7;160(1):11-7. doi: 10.7326/m13-1941. PMID: 24573662.

13. Sima JL, Perkins SM, Haggstrom DA. Primary care physician perceptions of adult survivors of childhood cancer. Journal of pediatric hematology/oncology. 2014 Mar;36(2):118-24. doi: $10.1097 / \mathrm{mph} .0000000000000061$. PMID: 24309612.

14. Guilcher GM, Fitzgerald C, Pritchard S. A questionnaire based review of long-term followup programs for survivors of childhood cancer in Canada. Pediatric blood \& cancer. 2009 Jan;52(1):113-5. doi: 10.1002/pbc.21701. PMID: 18821577.

15. Marr KC, Agha M, Sutradhar R, et al. Specialized survivor clinic attendance increases adherence to cardiomyopathy screening guidelines in adult survivors of childhood cancer. Journal of cancer survivorship : research and practice. 2017 Oct;11(5):614-23. doi: 10.1007/s11764-017-0634-z. PMID: 28785871. 
16. Nathan PC, Agha M, Pole JD, et al. Predictors of attendance at specialized survivor clinics in a population-based cohort of adult survivors of childhood cancer. Journal of cancer survivorship : research and practice. 2016 Aug;10(4):611-8. doi: 10.1007/s11764-016-0522-y. PMID: 26868681.

17. Oeffinger KC, Hudson MM, Mertens AC, et al. Increasing rates of breast cancer and cardiac surveillance among high-risk survivors of childhood Hodgkin lymphoma following a mailed, one-page survivorship care plan. Pediatric blood \& cancer. 2011 May;56(5):81824. doi: 10.1002/pbc.22696. PMID: 21370417.

18. Kadan-Lottick NS, Ross WL, Mitchell HR, et al. Randomized Trial of the Impact of Empowering Childhood Cancer Survivors With Survivorship Care Plans. Journal of the National Cancer Institute. 2018 Dec 1;110(12):1352-9. doi: 10.1093/jnci/djy057. PMID: 29771337.

19. Costello AG, Nugent BD, Conover N, et al. Shared Care of Childhood Cancer Survivors: A Telemedicine Feasibility Study. Journal of Adolescent \& Young Adult Oncology. 2017;6(4):535-41. doi:

10.1089/jayao.2017.0013. PMID: 126600837. Language: English. Entry Date: 20171213. Revision Date: 20181203. Publication Type: Article.

20. Yan AP, Chen Y, Henderson TO, et al. Adherence to Surveillance for Second Malignant Neoplasms and Cardiac Dysfunction in Childhood Cancer Survivors: A Childhood Cancer Survivor Study. Journal of Clinical Oncology. 2020;38(15):1711-22. doi: 10.1200/JCO.19.01825. PMID: 143226932. Language: English. Entry Date: In Process. Revision Date: 20201113. Publication Type: journal article. Journal Subset: Biomedical.

21. Berg CJ, Stratton E, Esiashvili N, et al. Young Adult Cancer Survivors' Experience with Cancer Treatment and Follow-Up Care and Perceptions of Barriers to Engaging in Recommended Care. Journal of cancer education : the official journal of the American Association for Cancer Education. 2016 Sep;31(3):430-42. doi: 10.1007/s13187-015-0853-9. PMID: 25948413.

22. Blaauwbroek R, Zwart N, Bouma M, et al. The willingness of general practitioners to be involved in the follow-up of adult survivors of childhood cancer. Journal of cancer survivorship : research and practice. 2007 Dec;1(4):292-7. doi: 10.1007/s11764-007-0032-z. PMID: 18648964.

23. Iyer NS, Mitchell HR, Zheng DJ, et al. Experiences with the survivorship care plan in primary care providers of childhood cancer survivors: a mixed methods approach.

Supportive care in cancer : official journal of the Multinational Association of Supportive Care in Cancer. 2017 May;25(5):1547-55. doi: 10.1007/s00520-016-3544-0. PMID: 28050709.

24. Michel G, Gianinazzi ME, Vetsch J, et al. Physicians' experience with follow-up care of childhood cancer survivors - challenges and needs. Swiss medical weekly. 2017;147:w14457. doi: 10.4414/smw.2017.14457. PMID: 28722079.

25. Hudson MM, Leisenring W, Stratton KK, et al. Increasing cardiomyopathy screening in at-risk adult survivors of pediatric malignancies: a randomized controlled trial. Journal of clinical oncology : official journal of the American Society of Clinical Oncology. 2014 Dec 10;32(35):3974-81. doi: 10.1200/jco.2014.57.3493. PMID: 25366684.

26. Murphy P, Levine A, Lerma T, et al. A portable survivorship care plan: a tool that helps educate and improve knowledge in childhood cancer survivors. Supportive care in cancer : official journal of the Multinational Association of Supportive Care in Cancer. 2021 Jan;29(1):16977. doi: 10.1007/s00520-020-05422-z. PMID: 32328773.

27. Pannier ST, Mann K, Warner EL, et al. Survivorship care plan experiences among childhood acute lymphoblastic leukemia patients and their families. BMC pediatrics. $2019 \mathrm{Apr}$ 13;19(1):111. doi: 10.1186/s12887-019-1464-0. PMID: 30979365.

28. Spain PD, Oeffinger KC, Candela J, et al. Response to a treatment summary and care plan among adult survivors of pediatric and young adult cancer. Journal of oncology practice. 2012 May;8(3):196-202. doi: 10.1200/jop.2011.000345. PMID: 22942816.

29. Shay LA, Parsons HM, Vernon SW. Survivorship Care Planning and Unmet Information and Service Needs Among Adolescent and Young Adult Cancer Survivors. Journal of adolescent and young adult oncology. 2017 Jun;6(2):327-32. doi: 10.1089/jayao.2016.0053. PMID: 28103126.

30. Hudson MM, Tyc VL, Srivastava DK, et al. Multi-component behavioral intervention to promote health protective behaviors in childhood cancer survivors: the protect study. Medical and pediatric oncology. 2002 Jul;39(1):2-1; discussion 2. doi: 10.1002/mpo.10071. PMID: 12116072.

31. Shi Q, Smith TG, Michonski JD, et al. Symptom burden in cancer survivors 1 year after diagnosis: 
a report from the American Cancer Society's Studies of Cancer Survivors. Cancer. 2011 Jun 15;117(12):2779-90. doi: 10.1002/cncr.26146. PMID: 21495026.

32. Nathan PC, Schiffman JD, Huang S, et al. Childhood cancer survivorship educational resources in North American pediatric hematology/oncology fellowship training programs: a survey study. Pediatric blood \& cancer. 2011 Dec 15;57(7):1186-90. doi: 10.1002/pbc.23214. PMID: 21674761.

33. Nielsen JD, Palshof T, Mainz J, et al. Randomised controlled trial of a shared care programme for newly referred cancer patients: bridging the gap between general practice and hospital. Quality \& safety in health care. 2003 Aug;12(4):263-72. doi: 10.1136/qhc.12.4.263. PMID: 12897359.

34. Ramirez AG, Muñoz E, Long Parma D, et al. Quality of life outcomes from a randomized controlled trial of patient navigation in Latina breast cancer survivors. Cancer medicine. 2020 Nov;9(21):7837-48. doi: 10.1002/cam4.3272. PMID: 32979042.

35. Grunfeld E, Fitzpatrick R, Mant D, et al. Comparison of breast cancer patient satisfaction with follow-up in primary care versus specialist care: results from a randomized controlled trial. The British journal of general practice : the journal of the Royal College of General Practitioners. 1999 Sep;49(446):705-10. PMID: 10756611.

36. Joubert J, Reid C, Joubert L, et al. Risk factor management and depression post-stroke: the value of an integrated model of care. Journal of clinical neuroscience : official journal of the Neurosurgical Society of Australasia. 2006 Jan;13(1):84-90. doi: 10.1016/j.jocn.2005.07.003. PMID: 16410202.

37. Dyer G, Gilroy N, Brown L, et al. What They Want: Inclusion of Blood and Marrow Transplantation Survivor Preference in the Development of Models of Care for Long-Term Health in Sydney, Australia. Biology of blood and marrow transplantation : journal of the American Society for Blood and Marrow Transplantation. 2016 Apr;22(4):731-43. doi: 10.1016/j.bbmt.2015.12.019. PMID: 26746819.

38. Boekhout AH, Maunsell E, Pond GR, et al. A survivorship care plan for breast cancer survivors: extended results of a randomized clinical trial. Journal of cancer survivorship : research and practice. $2015 \mathrm{Dec} ; 9(4): 683-91$. doi: 10.1007/s11764-015-0443-1. PMID: 25896265 .
39. Mao JJ, Bowman MA, Stricker CT, et al. Delivery of survivorship care by primary care physicians: the perspective of breast cancer patients. Journal of clinical oncology : official journal of the American Society of Clinical Oncology. 2009 Feb 20;27(6):933-8. doi: 10.1200/jco.2008.18.0679. PMID: 19139437.

40. Mani S, Khera N, Rybicki L, et al. Primary Care Physician Perspectives on Caring for Adult Survivors of Hematologic Malignancies and Hematopoietic Cell Transplantation. Clinical lymphoma, myeloma \& leukemia. 2020 Feb;20(2):70-7. doi: 10.1016/j.clml.2019.11.008. PMID: 31810888.

41. Stephens C, Klemanski D, Lustberg MB, et al. Primary care physician's confidence and coordination regarding the survivorship care for older breast cancer survivors. Supportive care in cancer : official journal of the Multinational Association of Supportive Care in Cancer. 2021 Jan;29(1):223-30. doi: 10.1007/s00520-02005448-3. PMID: 32338315.

42. Worster A, Bass MJ, Wood ML. Willingness to follow breast cancer. Survey of family physicians. Canadian family physician Medecin de famille canadien. 1996 Feb;42:263-8. PMID: 9222575.

43. Brennan ME, Butow P, Spillane AJ, et al. Survivorship care after breast cancer: follow-up practices of Australian health professionals and attitudes to a survivorship care plan. Asia-Pacific journal of clinical oncology. 2010 Jun;6(2):11625. doi: $10.1111 / \mathrm{j} .1743-7563.2010 .01286 . x$. PMID: 20565424.

44. Dixit N, Burke N, Rodriguez G, et al. Knowledge and self-efficacy for caring for breast and colon cancer survivors among safety net primary care providers. Supportive care in cancer : official journal of the Multinational Association of Supportive Care in Cancer. 2020 Oct;28(10):4923-31. doi: 10.1007/s00520-01905277-z. PMID: 32016600.

45. Forsythe LP, Alfano CM, Leach CR, et al. Who provides psychosocial follow-up care for posttreatment cancer survivors? A survey of medical oncologists and primary care physicians. Journal of clinical oncology : official journal of the American Society of Clinical Oncology. 2012 Aug 10;30(23):2897-905. doi: 10.1200/jco.2011.39.9832. PMID: 22778322.

46. Numico G, Pinto C, Gori S, et al. Clinical and organizational issues in the management of surviving breast and colorectal cancer patients: attitudes and feelings of medical oncologists. PloS one. 2014;9(7):e101170. doi: 
10.1371/journal.pone.0101170. PMID: 24983237.

47. Bober SL, Recklitis CJ, Campbell EG, et al. Caring for cancer survivors: a survey of primary care physicians. Cancer. 2009 Sep 15;115(18 Suppl):4409-18. doi: 10.1002/cncr.24590. PMID: 19731354.

48. Cheung WY, Aziz N, Noone AM, et al. Physician preferences and attitudes regarding different models of cancer survivorship care: a comparison of primary care providers and oncologists. Journal of cancer survivorship : research and practice. 2013 Sep;7(3):343-54. doi: 10.1007/s11764-013-0281-y. PMID: 23526165.

49. Buriak SE, Potter J, Bleckley MK. Using a predictive model of clinician intention to improve continuing health professional education on cancer survivorship. The Journal of continuing education in the health professions.

2015 Winter;35(1):57-64. doi: 10.1002/chp.21266. PMID: 25799973.

50. Chubak J, Tuzzio L, Hsu C, et al. Providing care for cancer survivors in integrated health care delivery systems: practices, challenges, and research opportunities. Journal of oncology practice. 2012 May;8(3):184-9. doi: 10.1200/jop.2011.000312. PMID: 22942814.

51. Etim AE, Schellhase KG, Sparapani R, et al. Effect of model of care delivery on mammography use among elderly breast cancer survivors. Breast cancer research and treatment. 2006 Apr;96(3):293-9. doi: 10.1007/s10549-0059141-4. PMID: 16538537.

52. Worster A, Wood ML, McWhinney IR, et al. Who provides follow-up care for patients with early breast cancer? Canadian family physician Medecin de famille canadien. 1995 Aug;41:1314-20. PMID: 7580380.

53. Choi Y, Radhakrishnan A, Mahabare D, et al. The Johns Hopkins Primary Care for Cancer Survivor Clinic: lessons learned in our first 4 years. Journal of cancer survivorship : research and practice. $2020 \mathrm{Feb}$;14(1):19-25. doi: 10.1007/s11764-019-00816-3. PMID: 31650473.

54. Mayer DK, Deal AM, Crane JM, et al. Using Survivorship Care Plans to Enhance Communication and Cancer Care Coordination: Results of a Pilot Study. Oncology nursing forum. 2016 Sep 1;43(5):636-45. doi: 10.1188/16.onf.636-645. PMID: 27541556.

55. Taylor K, Chivers P, Bulsara C, et al. Care After Lymphoma (CALy) trial: A phase II pilot pragmatic randomised controlled trial of a nurseled model of survivorship care. European journal of oncology nursing : the official journal of European Oncology Nursing Society. 2019
Jun;40:53-62. doi: 10.1016/j.ejon.2019.03.005. PMID: 31229207.

56. Parker PA, Banerjee SC, Matasar MJ, et al. Efficacy of a survivorship-focused consultation versus a time-controlled rehabilitation consultation in patients with lymphoma: A cluster randomized controlled trial. Cancer. 2018 Dec 1;124(23):4567-76. doi: 10.1002/cncr.31767. PMID: 30335188.

57. Jefford M, Gough K, Drosdowsky A, et al. A Randomized Controlled Trial of a Nurse-Led Supportive Care Package (SurvivorCare) for Survivors of Colorectal Cancer. The oncologist. 2016 Aug;21(8):1014-23. doi: 10.1634/theoncologist.2015-0533. PMID: 27306909.

58. Forsythe LP, Parry C, Alfano CM, et al. Use of survivorship care plans in the United States: associations with survivorship care. Journal of the National Cancer Institute. 2013 Oct 16;105(20):1579-87. doi: 10.1093/jnci/djt258. PMID: 24096621.

59. Jabson JM. Treatment summaries, follow-up care instructions, and patient navigation: could they be combined to improve cancer survivor's receipt of follow-up care? Journal of cancer survivorship : research and practice. 2015 Dec;9(4):692-8. doi: 10.1007/s11764-015-0444-0. PMID: 25764272.

60. Thom B, Boekhout AH, Corcoran S, et al. Advanced Practice Providers and Survivorship Care: They Can Deliver. Journal of oncology practice. 2019 Mar;15(3):e230-e7. doi: 10.1200/jop.18.00359. PMID: 30615587.

61. Nicolaije KA, Ezendam NP, Vos MC, et al. Impact of an Automatically Generated Cancer Survivorship Care Plan on Patient-Reported Outcomes in Routine Clinical Practice: Longitudinal Outcomes of a Pragmatic, Cluster Randomized Trial. Journal of clinical oncology : official journal of the American Society of Clinical Oncology. 2015 Nov 1;33(31):3550-9. doi: 10.1200/jco.2014.60.3399. PMID: 26304900.

62. Jeppesen MM, Ezendam NPM, Pijnenborg JMA, et al. The impact of the survivorship care plan on health care use: 2-year follow-up results of the ROGY care trial. Journal of cancer survivorship : research and practice. $2018 \mathrm{Feb}$;12(1):18-27. doi: 10.1007/s11764-017-0639-7. PMID: 28875470.

63. Nicolaije KA, Ezendam NP, Vos MC, et al. Oncology providers' evaluation of the use of an automatically generated cancer survivorship care plan: longitudinal results from the ROGY Care trial. Journal of cancer survivorship : research 
and practice. 2014 Jun;8(2):248-59. doi: 10.1007/s11764-013-0327-1. PMID: 24357226.

64. de Rooij BH, Ezendam NPM, Vos MC, et al. Patients' information coping styles influence the benefit of a survivorship care plan in the ROGY Care Trial: New insights for tailored delivery. Cancer. 2019 Mar 1;125(5):788-97. doi: 10.1002/cncr.31844. PMID: 30500067.

65. Brothers BM, Easley A, Salani R, et al. Do survivorship care plans impact patients' evaluations of care? A randomized evaluation with gynecologic oncology patients. Gynecologic oncology. 2013 Jun;129(3):554-8. doi: 10.1016/j.ygyno.2013.02.037. PMID: 23474344.

66. Majhail NS, Murphy E, Laud P, et al. Randomized controlled trial of individualized treatment summary and survivorship care plans for hematopoietic cell transplantation survivors. Haematologica. 2019 May;104(5):1084-92. doi: 10.3324/haematol.2018.203919. PMID: 30514795 .

67. Smith KC, Tolbert E, Hannum SM, et al. Comparing Web-Based Provider-Initiated and Patient-Initiated Survivorship Care Planning for Cancer Patients: A Randomized Controlled Trial. JMIR cancer. 2016 Aug 30;2(2):e12. doi: 10.2196/cancer.5947. PMID: 28410187.

68. Irene Su H, Stark S, Kwan B, et al. Efficacy of a web-based women's health survivorship care plan for young breast cancer survivors: a randomized controlled trial. Breast cancer research and treatment. $2019 \mathrm{Aug} ; 176(3): 579$ 89. doi: 10.1007/s10549-019-05260-6. PMID: 31054032 .

69. Hershman DL, Greenlee H, Awad D, et al. Randomized controlled trial of a clinic-based survivorship intervention following adjuvant therapy in breast cancer survivors. Breast cancer research and treatment. 2013 Apr;138(3):795806. doi: 10.1007/s10549-013-2486-1. PMID: 23542954.

70. Tevaarwerk AJ, Hocking WG, Buhr KA, et al. A randomized trial of immediate versus delayed survivorship care plan receipt on patient satisfaction and knowledge of diagnosis and treatment. Cancer. 2019 Mar 15;125(6):1000-7. doi: 10.1002/cncr.31875. PMID: 30690714.

71. Maly RC, Liang LJ, Liu Y, et al. Randomized Controlled Trial of Survivorship Care Plans Among Low-Income, Predominantly Latina Breast Cancer Survivors. Journal of clinical oncology : official journal of the American Society of Clinical Oncology. 2017 Jun 1;35(16):1814-21. doi: 10.1200/jco.2016.68.9497. PMID: 28418767.
72. Fang SY, Wang YL, Lu WH, et al. Long-term effectiveness of an E-based survivorship care plan for breast cancer survivors: A quasiexperimental study. Patient education and counseling. 2020 Mar;103(3):549-55. doi: 10.1016/j.pec.2019.09.012. PMID: 31558323.

73. Hua A, Sesto ME, Zhang X, et al. Impact of Survivorship Care Plans and Planning on Breast, Colon, and Prostate Cancer Survivors in a Community Oncology Practice. Journal of cancer education : the official journal of the American Association for Cancer Education. 2020 Apr;35(2):249-55. doi: 10.1007/s13187018-1457-y. PMID: 30610655.

74. Chrischilles EA, McDowell BD, Rubenstein L, et al. Survivorship care planning and its influence on long-term patient-reported outcomes among colorectal and lung cancer survivors: the CanCORS disease-free survivor follow-up study. Journal of cancer survivorship : research and practice. 2015 Jun;9(2):269-78. doi: 10.1007/s11764-014-0406-y. PMID: 25354481.

75. Palmer SC, Stricker CT, Panzer SL, et al. Outcomes and satisfaction after delivery of a breast cancer survivorship care plan: results of a multicenter trial. Journal of oncology practice. 2015 Mar;11(2):e222-9. doi: 10.1200/jop.2014.001404. PMID: 25784579.

76. Nissen MJ, Tsai ML, Blaes AH, et al. Effectiveness of treatment summaries in increasing breast and colorectal cancer survivors' knowledge about their diagnosis and treatment. Journal of cancer survivorship : research and practice. 2013 Jun;7(2):211-8. doi: 10.1007/s11764-012-0261-7. PMID: 23417167.

77. Mayer DK, Gerstel A, Walton AL, et al. Implementing survivorship care plans for colon cancer survivors. Oncology nursing forum. 2014 May;41(3):266-73. doi: 10.1188/14.onf.266-273. PMID: 24769591.

78. Nápoles AM, Santoyo-Olsson J, Chacón L, et al. Feasibility of a Mobile Phone App and Telephone Coaching Survivorship Care Planning Program Among Spanish-Speaking Breast Cancer Survivors. JMIR cancer. 2019 Jul 9;5(2):e13543. doi: 10.2196/13543. PMID: 31290395.

79. Brant JM, Blaseg K, Aders K, et al. Navigating the Transition From Cancer Care to Primary Care: Assistance of a Survivorship Care Plan. Oncology nursing forum. 2016 Nov 1;43(6):7109. doi: 10.1188/16.onf.710-719. PMID: 27768126.

80. Hébert J, Fillion L. Assessment of the feasibility and acceptability, and pre-test of the utility of an individualized survivorship care plan (ISCP) for 
women with endometrial cancers during the transition of the end of active treatment to cancer survivorship. Canadian oncology nursing journal $=$ Revue canadienne de nursing oncologique. 2017 Spring;27(2):153-63. doi: 10.5737/23688076272153163. PMID: 31148628 .

81. Nahm ES, Miller K, McQuaige M, et al. Testing the Impact of a Cancer Survivorship Patient Engagement Toolkit on Selected Health Outcomes. Oncology nursing forum. 2019 Sep 1;46(5):572-84. doi: 10.1188/19.onf.572-584. PMID: 31424456.

82. Morken CM, Tevaarwerk AJ, Swiecichowski AK, et al. Survivor and Clinician Assessment of Survivorship Care Plans for Hematopoietic Stem Cell Transplantation Patients: An Engineering, Primary Care, and Oncology Collaborative for Survivorship Health. Biology of blood and marrow transplantation : journal of the American Society for Blood and Marrow Transplantation. 2019 Jun;25(6):1240-6. doi: 10.1016/j.bbmt.2019.02.003. PMID: 30763727.

83. Campbell BH, Massey BL, Myers KB. Survivorship care plans for patients with head and neck cancer. Archives of otolaryngology-head \& neck surgery. 2012 Dec;138(12):1116-9. doi: 10.1001/jamaoto.2013.683. PMID: 23247230.

84. Ashing K, Serrano M, Weitzel J, et al. Towards developing a bilingual treatment summary and survivorship care plan responsive to Spanish language preferred breast cancer survivors. Journal of cancer survivorship : research and practice. 2014 Dec;8(4):580-94. doi: 10.1007/s11764-014-0363-5. PMID: 24859132.

85. Benci JL, Vachani CC, Hampshire MK, et al. Factors Influencing Delivery of Cancer Survivorship Care Plans: A National Patterns of Care Study. Frontiers in oncology. 2019;9:1577. doi: 10.3389/fonc.2019.01577. PMID: 32083018.

86. Jabson JM. Follow-up care instructions, treatment summaries, and cancer survivors' receipt of follow-up health care and late/long term effects. Supportive care in cancer : official journal of the Multinational Association of Supportive Care in Cancer. 2015 Jul;23(7):18516. doi: 10.1007/s00520-014-2532-5. PMID: 25471181.

87. Jabson JM, Bowen DJ. How do follow-up care instructions and treatment summaries relate to cancer survivors' cancer-related pain? Journal of pain and symptom management. 2014 Dec;48(6):1247-53. doi: 10.1016/j.jpainsymman.2014.03.004. PMID: 24780182.

88. Hawkins-Taylor C, Carson P, Anderson DG, et al. Survivorship Care Plans: Health Actions Taken and Satisfaction After Use. Oncology nursing forum. 2019 Sep 1;46(5):585-94. doi: 10.1188/19.onf.585-594. PMID: 31424453.

89. Rechis R, Beckjord EB, Nutt S. Potential benefits of treatment summaries for survivors' health and information needs: results from a LIVESTRONG survey. Journal of oncology practice. 2014 Jan;10(1):75-8. doi: 10.1200/jop.2013.000973. PMID: 24003173.

90. Blanch-Hartigan D, Chawla N, Beckjord EI, et al. Cancer survivors' receipt of treatment summaries and implications for patient-centered communication and quality of care. Patient education and counseling. 2015

Oct;98(10):1274-9. doi: 10.1016/j.pec.2015.06.005. PMID: 26146237.

91. Chahine S, Urquhart R. A cross-sectional population-based survey looking at the impact of cancer survivorship care plans on meeting the needs of cancer survivors in the posttreatment stage. Supportive Care in Cancer. 2019;27(10):3785-92. doi: 10.1007/s00520-01904685-5. PMID: 138431417. Language: English. Entry Date: 20191121. Revision Date: 20200930. Publication Type: journal article. Journal Subset: Biomedical.

92. Rosenberg SM, Ligibel JA, Meyerhardt JA, et al. Developing a Novel Model to Improve Research and care for Cancer Survivors: a Feasibility Study. Journal of cancer education : the official journal of the American Association for Cancer Education. 2019 Apr;34(2):229-33. doi: 10.1007/s13187-017-1291-7. PMID: 29052110.

93. Lyson HC, Haggstrom D, Bentz M, et al. Communicating Critical Information to Cancer Survivors: an Assessment of Survivorship Care Plans in Use in Diverse Healthcare Settings. Journal of cancer education : the official journal of the American Association for Cancer Education. 2020 Mar 4. doi: 10.1007/s13187020-01725-1. PMID: 32128714.

94. Rosales AR, Byrne D, Burnham C, et al. Comprehensive survivorship care with cost and revenue analysis. Journal of oncology practice. 2014 Mar;10(2):e81-5. doi: 10.1200/jop.2013.000945. PMID: 24065401.

95. Tarver WL, Robb BW, Haggstrom DA. Usefulness and Usability of a Personal Health Record and Survivorship Care Plan for Colorectal Cancer Survivors: Survey Study. JMIR cancer. 2019 Aug 20;5(2):e10692. doi: 10.2196/10692. PMID: 31432780. 
96. Frick MA, Vachani CC, Hampshire MK, et al. Survivorship after treatment of pancreatic cancer: insights via an Internet-based survivorship care plan tool. Journal of gastrointestinal oncology. 2017 Oct;8(5):890-6. doi: 10.21037/jgo.2017.08.07. PMID: 29184694.

97. Frick MA, Vachani CC, Hampshire MK, et al. Patient-Reported Survivorship Care Practices and Late Effects After Treatment of Hodgkin and Non-Hodgkin Lymphoma. JCO clinical cancer informatics. $2018 \mathrm{Dec} ; 2: 1-10$. doi: 10.1200/cci.18.00015. PMID: 30652594.

98. Birken SA, Mayer DK, Weiner BJ. Survivorship care plans: prevalence and barriers to use. Journal of cancer education : the official journal of the American Association for Cancer Education. 2013 Jun;28(2):290-6. doi: 10.1007/s13187-013-0469-x. PMID: 23526552.

99. Birken SA, Deal AM, Mayer DK, et al. Determinants of survivorship care plan use in US cancer programs. Journal of Cancer Education. 2014;29(4):720-7. doi: 10.1007/s13187-0140645-7. PMID: 109761532. Language: English. Entry Date: 20150814. Revision Date: 20200708. Publication Type: journal article.

100. Birken SA, Raskin S, Zhang Y, et al. Survivorship care plan implementation in US cancer programs: A national survey of cancer care providers. Journal of Cancer Education. 2019;34(3):614-22. doi: 10.1007/s13187-0181374-0. PMID: 2019-36497-028.

101. Merport A, Lemon SC, Nyambose J, et al. The use of cancer treatment summaries and care plans among Massachusetts physicians. Supportive Care in Cancer. 2012;20(7):1579-83. doi: 10.1007/s00520-012-1458-z. PMID: 104451414. Language: English. Entry Date: 20121102. Revision Date: 20200708. Publication Type: journal article.

102. Dittus KL, Sprague BL, Pace CM, et al. Primary Care Provider Evaluation of Cancer Survivorship Care Plans Developed for Patients in their Practice. Journal of general practice (Los Angeles, Calif). 2014 Jul;2(4):163. doi: 10.4172/2329-9126.1000163. PMID: 26451385.

103. Salz T, Oeffinger KC, Lewis PR, et al. Primary care providers' needs and preferences for information about colorectal cancer survivorship care. Journal of the American Board of Family Medicine : JABFM. 2012 Sep-Oct;25(5):635-51. doi: 10.3122/jabfm.2012.05.120083. PMID: 22956699.

104. Donohue S, Haine JE, Li Z, et al. Cancer Survivorship Care Plan Utilization and Impact on Clinical Decision-Making at Point-of-Care Visits with Primary Care: Results from an
Engineering, Primary Care, and Oncology Collaborative for Survivorship Health. Journal of cancer education : the official journal of the American Association for Cancer Education. 2019 Apr;34(2):252-8. doi: 10.1007/s13187-0171295-3. PMID: 29098650.

105. Donohue S, Sesto ME, Hahn DL, et al. Evaluating primary care providers' views on survivorship care plans generated by an electronic health record system. Journal of oncology practice. 2015 May;11(3):e329-35. doi: 10.1200/jop.2014.003335. PMID: 25804989.

106. Ashing KT, Lai L, Brown S, et al. Developing a treatment summary and survivorship care plan responsive to African-American breast cancer survivors. Psycho-oncology. 2016;25(6):729-31. doi: 10.1002/pon.3939. PMID: 116102489. Language: English. Entry Date: 20170316. Revision Date: 20180712. Publication Type: journal article.

107. Hamlish T, Liu L, Zhang Z, et al. Care Coordination for Breast Cancer Survivors in Urban Underserved Communities: Will Treatment Summaries and Survivorship Care Plans Be Enough? Journal of racial and ethnic health disparities. 2020 Jun;7(3):577-83. doi: 10.1007/s40615-019-00687-5. PMID: 31900747.

108. Phillips CS, Ko J, Porter J, et al. Does Receipt of a Survivorship Care Plan Enhance Survivors' Knowledge of Cancer History: A Retrospective Study. Journal of Oncology Navigation \& Survivorship. 2020;11(3):72-80. PMID: 141965522. Language: English. Entry Date: 20200302. Revision Date: 20200303. Publication Type: Article.

109. Chen Z, Koh PW, Ritter PL, et al. Dissecting an online intervention for cancer survivors: Four exploratory analyses of Internet engagement and its effects on health status and health behaviors.

Health Education \& Behavior. 2015;42(1):32-45. doi: 10.1177/1090198114550822. PMID: 201501449-005.

110. Risendal B, Dwyer A, Seidel R, et al. Adaptation of the chronic disease selfmanagement program for cancer survivors: feasibility, acceptability, and lessons for implementation. Journal of cancer education : the official journal of the American Association for Cancer Education. 2014 Dec;29(4):762-71. doi: 10.1007/s13187-014-0652-8. PMID: 24903138.

111. Sabatino SA, Thompson TD, Smith JL, et al. Receipt of cancer treatment summaries and follow-up instructions among adult cancer survivors: results from a national survey. Journal of cancer survivorship : research and practice. 
2013 Mar;7(1):32-43. doi: 10.1007/s11764-0120242-x. PMID: 23179495.

112. Shay LA, Schmidt S, Dioun SI, et al. Receipt of a survivorship care plan and self-reported health behaviors among cancer survivors. Journal of Cancer Survivorship. 2019;13(2):180-6. doi: 10.1007/s11764-019-00740-6. PMID: 136067650. Language: English. Entry Date: In Process. Revision Date: 20200415. Publication Type: journal article. Journal Subset: Biomedical.

113. Ostroff J, Ross S, Steinglass P, et al. Interest in and barriers to participation in multiple family groups among head and neck cancer survivors and their primary family caregivers. Family process. 2004 Jun;43(2):195-208. doi: 10.1111/j.1545-5300.2004.04302005.x. PMID: 15603503.

114. Chubak J, Aiello Bowles EJ, Tuzzio L, et al. Perspectives of cancer survivors on the role of different healthcare providers in an integrated delivery system. Journal of cancer survivorship : research and practice. 2014 Jun;8(2):229-38. doi: 10.1007/s11764-013-0335-1. PMID: 24352871.

115. Wiljer D, Urowitz S, Frasca E, et al. The role of a clinician-led reflective interview on improving self-efficacy in breast cancer survivors: a pilot study. Journal of cancer education : the official journal of the American Association for Cancer Education. 2010 Sep;25(3):457-63. doi: 10.1007/s13187-010-0103-0. PMID: 20383675.

116. Turner J, Yates P, Kenny L, et al. The ENHANCES study: a randomised controlled trial of a nurse-led survivorship intervention for patients treated for head and neck cancer. Supportive care in cancer : official journal of the Multinational Association of Supportive Care in Cancer. 2019 Dec;27(12):4627-37. doi: 10.1007/s00520-019-04748-7. PMID: 30941580.

117. Cairo J, Williams L, Bray L, et al. Evaluation of a Mobile Health Intervention to Improve Wellness Outcomes for Breast Cancer Survivors. Journal of patient-centered research and reviews. 2020 Fall;7(4):313-22. doi: 10.17294/23300698.1733. PMID: 33163551.

118. Cheung WY, Neville BA, Earle CC. Associations among cancer survivorship discussions, patient and physician expectations, and receipt of follow-up care. Journal of clinical oncology : official journal of the American Society of Clinical Oncology. 2010 May 20;28(15):2577-83. doi: 10.1200/jco.2009.26.4549. PMID: 20406932.
119. Belkora J, Katapodi M, Moore D, et al. Evaluation of a visit preparation intervention implemented in two rural, underserved counties of Northern California. Patient education and counseling. 2006 Dec;64(1-3):350-9. doi: 10.1016/j.pec.2006.03.017. PMID: 16904859.

120. Bazzell JL, Spurlock A, McBride M. Matching the unmet needs of cancer survivors to resources using a shared care model. Journal of cancer education : the official journal of the American Association for Cancer Education. 2015 Jun;30(2):312-8. doi: 10.1007/s13187-014-07089. PMID: 25103849.

121. Playdon M, Ferrucci LM, McCorkle R, et al. Health information needs and preferences in relation to survivorship care plans of long-term cancer survivors in the American Cancer Society's Study of Cancer Survivors-I. Journal of cancer survivorship : research and practice. 2016 Aug;10(4):674-85. doi: 10.1007/s11764-0150513-4. PMID: 26744339.

122. de Rooij BH, Park ER, Perez GK, et al. Cluster Analysis Demonstrates the Need to Individualize Care for Cancer Survivors. The oncologist. 2018 Dec;23(12):1474-81. doi: 10.1634/theoncologist.2017-0558. PMID: 29739897.

123. Brennan ME, Butow P, Spillane AJ, et al. Patient-reported quality of life, unmet needs and care coordination outcomes: Moving toward targeted breast cancer survivorship care planning. Asia-Pacific journal of clinical oncology. 2016 Jun;12(2):e323-31. doi: 10.1111/ajco.12254. PMID: 25244662.

124. Ford ME, Sterba KR, Armeson K, et al. Factors Influencing Adherence to Recommended Colorectal Cancer Surveillance: Experiences and Behaviors of Colorectal Cancer Survivors. Journal of Cancer Education. 2019;34(5):938-49. doi: 10.1007/s13187-018-1398-5. PMID: 139028875. Language: English. Entry Date: 20200701. Revision Date: 20201002. Publication Type: journal article. Journal Subset: Biomedical.

125. Lim J-W, Paek M-S. The relationship between communication and health-related quality of life in survivorship care for Chinese-American and Korean-American breast cancer survivors. Supportive Care in Cancer. 2013;21(4):1157-66. doi: 10.1007/s00520-012-1641-2. PMID: 104242876. Language: English. Entry Date: 20130927. Revision Date: 20200708. Publication Type: journal article. 


\section{List of Acronyms}

\begin{tabular}{|c|c|}
\hline 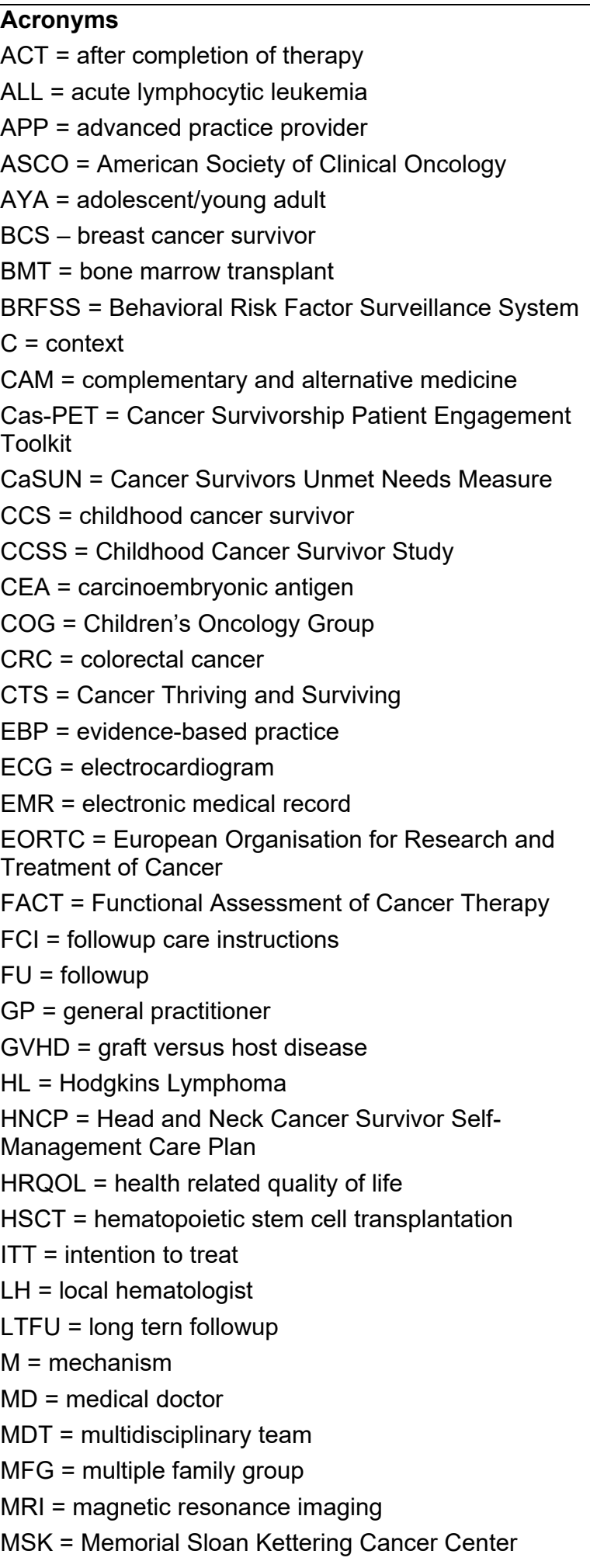 & $\begin{array}{l}\mathrm{NCI}=\text { National Cancer Institute } \\
\mathrm{NP}=\text { nurse practitioner } \\
\mathrm{NSW} \text { = New South Wales } \\
\mathrm{O}=\text { outcome } \\
\text { OR = odds ratio } \\
\mathrm{PA}=\text { physician assistant } \\
\text { PCCS = Primary Care for Cancer Survivors clinic } \\
\text { PCP = primary care provider } \\
\text { PHR = personal health record } \\
\text { PN = patient navigation } \\
\text { QoL = quality of life } \\
\text { RAV = risk adapted visit } \\
\text { RHIT = Registered Health Information Technologist } \\
\text { ROGY = Registration system Oncological Gynecology } \\
\text { RVU = relative value units } \\
\text { SAM = self-assessment and management } \\
\text { SC = standard care } \\
\text { SCP = survivorship care plan } \\
\text { SEER = Surveillance, Epidemiology, and End Results } \\
\text { SES = socioeconomic status } \\
\text { SMART = Specific, Measurable, Achievable, Relevant, } \\
\text { Timely } \\
\text { TC = treatment center } \\
\text { TS = treatment summary } \\
\text { TSSCP-S = Treatment Summary and Survivorship } \\
\text { Care Plan } \\
\text { US = United States }\end{array}$ \\
\hline
\end{tabular}




\section{Appendix D. List of References Used To Inform the Contextual Questions and Initial Program Theory}

Childhood Cancer Survivorship: Improving Care and Quality of Life, ed. W.S. Hewitt M, Simone JV. 2003: Institute of Medicine, National Research Council.

Aune, G.J., Health-care delivery for long-term survivors of childhood cancer. Lancet, 2017. 390(10112): p. 2545.

Avila, J.C., et al., Preventive services use among female survivors of adolescent and young adult cancer. J Cancer Surviv, 2017. 11(2): p. 167-174.

Aziz, N.M., et al., Comprehensive long-term followup programs for pediatric cancer survivors. Cancer, 2006. 107(4): p. 841-8.

Berg, C.J., et al., Pilot results of an online intervention targeting health promoting behaviors among young adult cancer survivors. Psychooncology, 2014. 23(10): p. 1196-9.

Berg, C.J., et al., Young Adult Cancer Survivors' Experience with Cancer Treatment and FollowUp Care and Perceptions of Barriers to Engaging in Recommended Care. J Cancer Educ, 2016. 31(3): p. 430-42.

Blaauwbroek, R., et al., Family doctor-driven followup for adult childhood cancer survivors supported by a web-based survivor care plan. J Cancer Surviv, 2012. 6(2): p. 163-71.

Blaauwbroek, R., et al., Shared care by paediatric oncologists and family doctors for long-term follow-up of adult childhood cancer survivors: a pilot study. Lancet Oncol, 2008. 9(3): p. 232-8.

Bowers, D.C., et al., Survey of long-term follow-up programs in the United States for survivors of childhood brain tumors. Pediatr Blood Cancer, 2009. 53(7): p. 1295-301.

Casillas, J., et al., Impact of insurance type on survivor-focused and general preventive health care utilization in adult survivors of childhood cancer: the Childhood Cancer Survivor Study (CCSS). Cancer, 2011. 117(9): p. 1966-75.

Casillas, J., et al., Transitioning childhood cancer survivors to adult-centered healthcare: insights from parents, adolescent, and young adult survivors. Psychooncology, 2010. 19(9): p. 98290.
Cherven, B., et al., Knowledge and risk perception of late effects among childhood cancer survivors and parents before and after visiting a childhood cancer survivor clinic. J Pediatr Oncol Nurs, 2014. 31(6): p. 339-49.

Choi, Y., et al., The Johns Hopkins Primary Care for Cancer Survivor Clinic: lessons learned in our first 4 years. J Cancer Surviv, 2020. 14(1): p. 1925.

Chubak, J., et al., Providing care for cancer survivors in integrated health care delivery systems: practices, challenges, and research opportunities. J Oncol Pract, 2012. 8(3): p. 184-9.

Coccia, P.F., et al., Adolescent and Young Adult Oncology, Version 2.2018, NCCN Clinical Practice Guidelines in Oncology. J Natl Compr Canc Netw, 2018. 16(1): p. 66-97.

Coccia, P.F., et al., Adolescent and young adult oncology. Clinical practice guidelines in oncology. J Natl Compr Canc Netw, 2012. 10(9): p. $1112-50$.

Cox, C.L., et al., Determinants of Mammography Screening Participation in Adult Childhood Cancer Survivors: Results From the Childhood Cancer Survivor Study. Oncol Nurs Forum, 2009. 36(3): p. 335-344.

Crabtree, B.F., et al., Cancer Survivorship Care Roles for Primary Care Physicians. Ann Fam Med, 2020. 18(3): p. 202-209.

Del Giudice, M.E., et al., Primary care physicians' views of routine follow-up care of cancer survivors. J Clin Oncol, 2009. 27(20): p. 333845.

Duffey-Lind, E.C., et al., Transitioning to survivorship: a pilot study. J Pediatr Oncol Nurs, 2006. 23(6): p. 335-43.

Earle, C.C., Failing to plan is planning to fail: improving the quality of care with survivorship care plans. J Clin Oncol, 2006. 24(32): p. 51126.

Earle, E.A., et al., Follow-up care for childhood cancer survivors: a focus group analysis. Eur J Cancer, 2005. 41(18): p. 2882-6. 
Essig, S., et al., Follow-up programs for childhood cancer survivors in Europe: a questionnaire survey. PLoS One, 2012. 7(12): p. e53201.

Fardell, J.E., et al., Transition of childhood cancer survivors to adult care: The survivor perspective. Pediatr Blood Cancer, 2017. 64(6).

Feldman-Stewart, D., M.D. Brundage, and C. Tishelman, A conceptual framework for patientprofessional communication: an application to the cancer context. Psychooncology, 2005. 14(10): p. 801-9; discussion 810-1.

Gibson, T.M., G.T. Armstrong, and L.L. Robison, Reply to Perceptions of future health and cancer risk in adult survivors of childhood cancer: Implications for engagement in follow-up care. Cancer, 2019. 125(6): p. 1009-1010.

Halpern, M.T. and K.E. Argenbright, Evaluation of effectiveness of survivorship programmes: how to measure success? Lancet Oncol, 2017. 18(1): p. e51-e59.

Henderson, T.O. and K.C. Oeffinger, Enhancing Health Care of Survivors of Childhood Cancer With Tailored Education. J Clin Oncol, 2015. 33(33): p. 3849-50.

Henderson, T.O., D.L. Friedman, and A.T. Meadows, Childhood cancer survivors: transition to adultfocused risk-based care. Pediatrics, 2010. 126(1): p. 129-36.

Henderson, T.O., et al., Physician preferences and knowledge gaps regarding the care of childhood cancer survivors: a mailed survey of pediatric oncologists. J Clin Oncol, 2010. 28(5): p. 878 83.

Hohmann, N.S., et al., Patient perspectives on primary care and oncology care coordination in the context of multiple chronic conditions: A systematic review. Res Social Adm Pharm, 2020. 16(8): p. 1003-1016.

Hudson, M.M., A model for care across the cancer continuum. Cancer, 2005. 104(11 Suppl): p. 2638-42.

Konsler, G.K. and G.R. Jones, Transition issues for survivors of childhood cancer and their healthcare providers. Cancer Pract, 1993. 1(4): p. 319-24.

Landier, W., W.H. Wallace, and M.M. Hudson, Long-term follow-up of pediatric cancer survivors: education, surveillance, and screening. Pediatr Blood Cancer, 2006. 46(2): p. 149-58.
Langer, T., G. Henze, and J.D. Beck, Basic methods and the developing structure of a late effects surveillance system (LESS) in the long-term follow-up of pediatric cancer patients in Germany. For the German Late Effects Study Group in the German Society Pediatric Oncology and Hematology (GPOH). Med Pediatr Oncol, 2000. 34(5): p. 348-51.

Long-term follow-up care for pediatric cancer survivors. Pediatrics, 2009. 123(3): p. 906-15.

Marjerrison, S. and R.D. Barr, Unmet Survivorship Care Needs of Adolescent and Young Adult Cancer Survivors. JAMA Netw Open, 2018. 1(2): p. e180350.

McLaughlin, S., et al., Here, There and Nowhere: Following Adult Survivors of Childhood Cancer A Case Report of Recurrent Osteosarcoma in a Young Adult. R I Med J (2013), 2016. 99(8): p. 25-6.

Meeske, K.A. and M.B. Nelson, The role of the longterm follow-up clinic in discovering new emerging late effects in adult survivors of childhood cancer. J Pediatr Oncol Nurs, 2008. 25(4): p. 213-9.

Michel, G., et al., Evidence-based recommendations for the organization of long-term follow-up care for childhood and adolescent cancer survivors: a report from the PanCareSurFup Guidelines Working Group. J Cancer Surviv, 2019. 13(5): p. 759-772.

Nagel, K., et al., The development of an off-therapy needs questionnaire and protocol for survivors of childhood cancer. J Pediatr Oncol Nurs, 2002. 19(6): p. 229-33.

Nathan, P.C., et al., Family physician preferences and knowledge gaps regarding the care of adolescent and young adult survivors of childhood cancer. J Cancer Surviv, 2013. 7(3): p. 275-82.

Nathan, P.C., et al., Health behaviors, medical care, and interventions to promote healthy living in the Childhood Cancer Survivor Study cohort. J Clin Oncol, 2009. 27(14): p. 2363-73.

Nekhlyudov, L., et al., Developing a Quality of Cancer Survivorship Care Framework: Implications for Clinical Care, Research, and Policy. J Natl Cancer Inst, 2019. 111(11): p. 1120-1130.

Nekhlyudov, L., Integrating primary care in cancer survivorship programs: models of care for a growing patient population. Oncologist, 2014. 19(6): p. 579-82. 
Nekhlyudov, L., M. O'Malley D, and S.V. Hudson, Integrating primary care providers in the care of cancer survivors: gaps in evidence and future opportunities. Lancet Oncol, 2017. 18(1): p. e30e38.

Oeffinger, K.C. and M.S. McCabe, Models for delivering survivorship care. J Clin Oncol, 2006. 24(32): p. 5117-24.

Oeffinger, K.C. and W.H. Wallace, Barriers to follow-up care of survivors in the United States and the United Kingdom. Pediatr Blood Cancer, 2006. 46(2): p. 135-42.

Oeffinger, K.C., et al., Programs for adult survivors of childhood cancer. J Clin Oncol, 1998. 16(8): p. 2864-7.

O'Malley, D., et al., Learning the landscape: implementation challenges of primary care innovators around cancer survivorship care. $\mathrm{J}$ Cancer Surviv, 2017. 11(1): p. 13-23.

Prasad, P.K., T. Bowles, and D.L. Friedman, Is there a role for a specialized follow-up clinic for survivors of pediatric cancer? Cancer Treat Rev, 2010. 36(4): p. 372-6.

Reed, S.C., A.H. Partridge, and L. Nekhlyudov, Shared Medical Appointments in Cancer Survivorship Care: A Review of the Literature. J Oncol Pract, 2015. 11(1): p. 6-11.

Richardson, R.C., M.B. Nelson, and K. Meeske, Young adult survivors of childhood cancer: attending to emerging medical and psychosocial needs. J Pediatr Oncol Nurs, 1999. 16(3): p. 13644.
Ristovski-Slijepcevic, S., et al., A cross-Canada survey of clinical programs for the care of survivors of cancer in childhood and adolescence. Paediatr Child Health, 2009. 14(6): p. 375-8.

Sadak, K.T., Associates of Engagement in AdultOriented Follow-Up Care for Childhood Cancer Survivors. J Adolesc Health, 2017. 60(2): p. 127128.

Schulmeister, L., Childhood cancer survival: the good news and the not-so-good news. Clin J Oncol Nurs, 2004. 8(1): p. 11.

Schwartz, C.L., Creating a bridge for transition: From pediatric cancer survival to life-long, risk-based health care of the adult cancer survivor. Cancer, 2020. 126(3): p. 473-476.

Seehusen, D.A., D. Baird, and D. Bode, Primary care of adult survivors of childhood cancer. Am Fam Physician, 2010. 81(10): p. 1250-5.

Study: Many survivors of childhood cancer lack follow-up care. Cancer, 2017. 123(3): p. 371.

Suh, E., et al., General internists' preferences and knowledge about the care of adult survivors of childhood cancer: a cross-sectional survey. Ann Intern Med, 2014. 160(1): p. 11-7.

Tonorezos, E.S. and K.C. Oeffinger, Specialty care for adult survivors of childhood cancer. Cancer, 2015. 121(24): p. 4279-81.

Weaver, S.J., et al., Unpacking Care Coordination Through a Multiteam System Lens: A Conceptual Framework and Systematic Review. Med Care, 2018. 56(3): p. 247-259. 


\section{Appendix E. List of Survivorship Resources Available to Cancer Survivors, Their Families, and Their Medical Care Providers}

Table D-1. List of survivorship resources available to cancer survivors, their families, and their medical care providers

\begin{tabular}{|c|c|c|c|c|}
\hline Resource & Link & $\begin{array}{l}\text { For Cancer } \\
\text { Survivors }\end{array}$ & $\begin{array}{l}\text { For } \\
\text { Providers }\end{array}$ & Description \\
\hline $\begin{array}{l}\text { American Cancer Society: } \\
\text { Survivorship: During and After } \\
\text { Treatment }\end{array}$ & $\begin{array}{l}\text { https://www.cancer.org/treatment/survivor } \\
\text { ship-during-and-after-treatment.html }\end{array}$ & Yes & No & $\begin{array}{l}\text { Information and tips on staying active } \\
\text { and healthy during and after cancer } \\
\text { treatment for cancer survivors. There is } \\
\text { also information on dealing with the } \\
\text { possibility of cancer recurrence, and } \\
\text { stories about other people whose lives } \\
\text { have been touched by cancer. }\end{array}$ \\
\hline $\begin{array}{l}\text { American Cancer Society: Tools } \\
\text { for Cancer Survivors and } \\
\text { Caregivers }\end{array}$ & $\begin{array}{l}\text { https://www.cancer.org/health-care- } \\
\text { professionals/national-cancer- } \\
\text { survivorship-resource-center/tools-for- } \\
\text { cancer-survivors-and-caregivers.html }\end{array}$ & Yes & No & $\begin{array}{l}\text { Guidance documents for cancer } \\
\text { survivors, including "Life After Treatment } \\
\text { Guide" and a "Life After Treatment } \\
\text { Guide" for Native Americans and Alaska } \\
\text { Natives. }\end{array}$ \\
\hline $\begin{array}{l}\text { American Cancer Society: Tools } \\
\text { for Health Care Professionals }\end{array}$ & $\begin{array}{l}\text { https://www.cancer.org/health-care- } \\
\text { professionals/national-cancer- } \\
\text { survivorship-resource-center/tools-for- } \\
\text { health-care-professionals.html }\end{array}$ & No & Yes & $\begin{array}{l}\text { Cancer survivorship care tools and } \\
\text { resources for providers, organizations, } \\
\text { and communities. }\end{array}$ \\
\hline $\begin{array}{l}\text { American Institute for Cancer } \\
\text { Research }\end{array}$ & $\begin{array}{l}\text { https://www.aicr.org/?gclid=Cl6fkJ6Y1rEC } \\
\text { FYao4AodiE0Aqw }\end{array}$ & No & Yes & $\begin{array}{l}\text { Monthly updates with resources, tools, } \\
\text { and guidance for healthcare } \\
\text { professionals }\end{array}$ \\
\hline $\begin{array}{l}\text { American Society of Clinical } \\
\text { Oncology: Cancer.Net* }\end{array}$ & $\begin{array}{l}\text { https://www.cancer.net/survivorship/surviv } \\
\text { orship-resources }\end{array}$ & Yes & No & $\begin{array}{l}\text { Cancer.Net provides timely, } \\
\text { comprehensive information to help } \\
\text { patients and families make informed } \\
\text { health care decisions. }\end{array}$ \\
\hline $\begin{array}{l}\text { American Society of Clinical } \\
\text { Oncology: Survivorship } \\
\text { Compendium }\end{array}$ & $\begin{array}{l}\text { https://www.asco.org/practice- } \\
\text { policy/cancer-care-initiatives/prevention- } \\
\text { survivorship/survivorship/survivorship- } \\
\underline{\text { compendium }}\end{array}$ & No & Yes & $\begin{array}{l}\text { A repository of tools and resources for } \\
\text { oncology providers to implement or } \\
\text { improve survivorship care within their } \\
\text { practices. The compendium serves as an } \\
\text { accompaniment to the educational } \\
\text { opportunities and clinical-guidance } \\
\text { ASCO offers on survivorship care. }\end{array}$ \\
\hline Cancer + Careers & https://www.cancerandcareers.org/en & Yes & No & $\begin{array}{l}\text { Provide resources for cancer patients } \\
\text { and survivors on thriving in the } \\
\text { workplace, providing expert advice, } \\
\text { interactive tools, and educational events. }\end{array}$ \\
\hline
\end{tabular}




\begin{tabular}{|c|c|c|c|c|}
\hline Resource & Link & $\begin{array}{l}\text { For Cancer } \\
\text { Survivors }\end{array}$ & $\begin{array}{l}\text { For } \\
\text { Providers }\end{array}$ & Description \\
\hline Cancer Care & https://www.cancercare.org/ & Yes & No & $\begin{array}{l}\text { Providing comprehensive services } \\
\text { including case management, counseling, } \\
\text { and support groups over the phone, } \\
\text { online, and in-person, educational } \\
\text { workshops, publications and financial } \\
\text { and co-payment assistance. }\end{array}$ \\
\hline $\begin{array}{l}\text { Cancer Financial Assistance } \\
\text { Coalition }\end{array}$ & https://www.cancerfac.org/ & Yes & No & $\begin{array}{l}\text { Coalition of organizations helping cancer } \\
\text { patients find financial and practical help } \\
\text { (e.g., home care, housing, fertility } \\
\text { assistance, genetic testing). }\end{array}$ \\
\hline Cancer Match & https://cancermatch.com/ & Yes & No & $\begin{array}{l}\text { A cancer survivor networking and dating } \\
\text { website. }\end{array}$ \\
\hline Cancer Support Community & https://www.cancersupportcommunity.org/ & Yes & No & $\begin{array}{l}\text { Non-profit network of support } \\
\text { organizations, hospitals, and clinic } \\
\text { partnerships delivering free support and } \\
\text { navigation services for cancer patients } \\
\text { and families. }\end{array}$ \\
\hline $\begin{array}{l}\text { Cancer Support Community: } \\
\text { Gilda's Club }\end{array}$ & https://www.cancersupportcommunity.org/ & Yes & No & $\begin{array}{l}\text { A member of the Cancer Support } \\
\text { Community, offers support through } \\
\text { counseling, connecting patients with a } \\
\text { wider patient and caregiver community, } \\
\text { and professionally led programs. }\end{array}$ \\
\hline Cancer SurvivorLink & https://www.cancersurvivorlink.org/ & Yes & Yes & $\begin{array}{l}\text { A patient-centric communication tool and } \\
\text { mechanism for cancer survivors } \\
\text { providing repository for key health } \\
\text { documents and ability to share their } \\
\text { online health records with providers. }\end{array}$ \\
\hline $\begin{array}{l}\text { Centers for Disease Control \& } \\
\text { Prevention: Cancer Survivors }\end{array}$ & $\begin{array}{l}\text { https://www.cdc.gov/cancer/survivors/inde } \\
\underline{\text { x.htm }}\end{array}$ & Yes & Yes & $\begin{array}{l}\text { CDC website with guidance and } \\
\text { information for cancer patients and } \\
\text { survivors, caregivers, and health care } \\
\text { providers. }\end{array}$ \\
\hline Children's Oncology Group † & http://survivorshipguidelines.org/ & No & Yes & $\begin{array}{l}\text { Children's Oncology Group guideline is a } \\
\text { resource for healthcare professionals } \\
\text { who provide ongoing care to survivors of } \\
\text { pediatric malignancies. }\end{array}$ \\
\hline $\begin{array}{l}\text { Dana-Farber Cancer Institute: } \\
\text { Continuing Medical Education }\end{array}$ & $\begin{array}{l}\text { https://www.dana-farber.org/for- } \\
\text { physicians/education-and- } \\
\text { training/continuing-medical-education/ }\end{array}$ & No & Yes & $\begin{array}{l}\text { Offering continuing medical education } \\
\text { courses, sharing advances in cancer } \\
\text { research and treatment with the } \\
\text { healthcare community. }\end{array}$ \\
\hline
\end{tabular}




\begin{tabular}{|c|c|c|c|c|}
\hline Resource & Link & $\begin{array}{l}\text { For Cancer } \\
\text { Survivors }\end{array}$ & $\begin{array}{l}\text { For } \\
\text { Providers }\end{array}$ & Description \\
\hline $\begin{array}{l}\text { George Washington University } \\
\text { Cancer Center: Advancing } \\
\text { Patient-Centered Cancer } \\
\text { Survivorship Care Toolkit }\end{array}$ & $\begin{array}{l}\text { https://smhs.gwu.edu/cancercontroltap/res } \\
\text { ources/advancing-patient-centered- } \\
\underline{\text { cancer-survivorship-care-toolkit }}\end{array}$ & No & Yes & $\begin{array}{l}\text { A toolkit to support training and technical } \\
\text { assistance from Comprehensive Cancer } \\
\text { Control Programs/Coalitions to health } \\
\text { care providers/organizations to improve } \\
\text { patient-centered cancer survivorship } \\
\text { care in their state, tribe or territory. }\end{array}$ \\
\hline Job Accommodation Network & https://askjan.org/ & Yes & No & $\begin{array}{l}\text { Offers guidance on workplace } \\
\text { accommodations and disability } \\
\text { employment issues. }\end{array}$ \\
\hline JourneyForward & https://www.journeyforward.org/ & Yes & No & $\begin{array}{l}\text { Health website offering information on } \\
\text { important health related topics, including } \\
\text { cancer symptom management and post- } \\
\text { treatment care. }\end{array}$ \\
\hline LIVESTRONG & https://www.livestrong.org/ & Yes & No & $\begin{array}{l}\text { Non-profit organization providing support } \\
\text { for people affected by cancer. Website } \\
\text { offers contact information and list of } \\
\text { available programs for cancer patients } \\
\text { and survivors. }\end{array}$ \\
\hline $\begin{array}{l}\text { LIVESTRONG Survivorship } \\
\text { Centers of Excellence }\end{array}$ & $\begin{array}{l}\text { https://www.livestrong.org/who-we- } \\
\text { are/news/livestrong-survivorship-centers- } \\
\text { excellence-network-celebrates-10-years- } \\
\text { success }\end{array}$ & Yes & No & $\begin{array}{l}\text { Website celebrates the LIVESTRONG } \\
\text { Survivorship Centers of Excellence's } 10 \\
\text { year anniversary and summarizes the } \\
\text { work done by the centers and } \\
\text { foundation. }\end{array}$ \\
\hline $\begin{array}{l}\text { National Cancer Survivors Day } \\
\text { Foundation }\end{array}$ & https://ncsd.org/ & Yes & No & $\begin{array}{l}\text { The nonprofit National Cancer Survivors } \\
\text { Day Foundation provides free guidance, } \\
\text { education, and networking to hospitals, } \\
\text { support groups, and other cancer-related } \\
\text { organizations that host NCSD events in } \\
\text { their communities. }\end{array}$ \\
\hline $\begin{array}{l}\text { National Coalition for Cancer } \\
\text { Survivorship }\end{array}$ & https://canceradvocacy.org/ & Yes & No & $\begin{array}{l}\text { A survivor-led cancer advocacy } \\
\text { organization, advocating for research, } \\
\text { policy, and providing tools for self- } \\
\text { advocacy. }\end{array}$ \\
\hline $\mathrm{NIH} / \mathrm{NCl}$ Cancer Survivorship & $\begin{array}{l}\text { https://www.cancer.gov/about- } \\
\text { cancer/coping/survivorship }\end{array}$ & Yes & No & $\begin{array}{l}\text { Offers information for cancer patients to } \\
\text { help cope with the transition to post- } \\
\text { treatment. }\end{array}$ \\
\hline
\end{tabular}




\begin{tabular}{|c|c|c|c|c|}
\hline Resource & Link & $\begin{array}{l}\text { For Cancer } \\
\text { Survivors }\end{array}$ & $\begin{array}{l}\text { For } \\
\text { Providers }\end{array}$ & Description \\
\hline $\begin{array}{l}\mathrm{NIH} / \mathrm{NCl} \text { : Cancer-Related Post- } \\
\text { traumatic Stress (PDQ®)-Patient } \\
\text { Version }\end{array}$ & $\begin{array}{l}\text { https://www.cancer.gov/about- } \\
\text { cancer/coping/survivorship/new- } \\
\text { normal/ptsd-pdq }\end{array}$ & Yes & No & $\begin{array}{l}\text { The National Cancer Institute's Physician } \\
\text { Data Query on post-traumatic stress } \\
\text { targeted for patients, providing } \\
\text { summaries on the latest published } \\
\text { information on cancer prevention, } \\
\text { detection, genetics, treatment, supportive } \\
\text { care, and complementary and alternative } \\
\text { medicine. }\end{array}$ \\
\hline $\begin{array}{l}\mathrm{NIH} / \mathrm{NCl} \text { : Office of Cancer } \\
\text { Survivorship }\end{array}$ & $\begin{array}{l}\text { https://cancercontrol.cancer.gov/ocs/resou } \\
\text { rces }\end{array}$ & Yes & Yes & $\begin{array}{l}\text { Offers resources and information for } \\
\text { many different audiences, including } \\
\text { survivors and caregivers, researchers, } \\
\text { health care professionals, and } \\
\text { advocates. }\end{array}$ \\
\hline $\begin{array}{l}\mathrm{NIH} / \mathrm{NCl} \text { : Office of Cancer } \\
\text { Survivorship }\end{array}$ & $\begin{array}{l}\text { https://cancercontrol.cancer.gov/ocs/resou } \\
\text { rces/survivors/follow-up-medical-care }\end{array}$ & No & Yes & $\begin{array}{l}\text { Links to resources for patients to assist } \\
\text { in dialogue with physicians on followup } \\
\text { care. }\end{array}$ \\
\hline NIH/NCI: SEER-CAHPS & $\begin{array}{l}\text { https://healthcaredelivery.cancer.gov/seer } \\
\text {-cahps/ }\end{array}$ & No & Yes* & $\begin{array}{l}\text { A resource for quality of cancer care } \\
\text { research based on a linkage between the } \\
\text { NCl's Surveillance, Epidemiology and } \\
\text { End Results (SEER) cancer registry data } \\
\text { and the Centers for Medicare \& Medicaid } \\
\text { Services' (CMS) Medicare Consumer } \\
\text { Assessment of Healthcare Providers and } \\
\text { Systems (CAHPS®) patient surveys. }\end{array}$ \\
\hline $\begin{array}{l}\text { Northwestern University: } \\
\text { Oncofertility }\end{array}$ & $\begin{array}{l}\text { https://www.nm.org/healthbeat/medical- } \\
\text { advances/oncofertility }\end{array}$ & Yes & No & $\begin{array}{l}\text { Offers resources on oncofertility, such as } \\
\text { options for fertility treatment and } \\
\text { methods to preserve a child's fertility } \\
\text { before cancer treatment. }\end{array}$ \\
\hline $\begin{array}{l}\text { OncoLink: OncoLife Survivorship } \\
\text { Care Plan }\end{array}$ & https://oncolife.oncolink.org/ & Yes & No & $\begin{array}{l}\text { A program providing cancer survivors } \\
\text { with information regarding the health } \\
\text { risks they face as a result of cancer } \\
\text { therapies, and resources to develop a } \\
\text { cancer survivorship care plan. }\end{array}$ \\
\hline $\begin{array}{l}\text { Passport for Care (with the } \\
\text { Children's Oncology Group) }\end{array}$ & $\begin{array}{l}\text { https://cancersurvivor.passportforcare.org/ } \\
\text { en/ }\end{array}$ & Yes & Yes & $\begin{array}{l}\text { A free online resource tool for childhood } \\
\text { cancer survivors allowing them to access } \\
\text { their treatment summary and followup } \\
\text { care recommendations. }\end{array}$ \\
\hline Patient Advocate Foundation & https://www.patientadvocate.org/ & Yes & No & $\begin{array}{l}\text { A non-profit organization offering case } \\
\text { management services and financial aid } \\
\text { to Americans with chronic, life } \\
\text { threatening, and debilitating illnesses. }\end{array}$ \\
\hline
\end{tabular}




\begin{tabular}{|c|c|c|c|c|}
\hline Resource & Link & $\begin{array}{l}\text { For Cancer } \\
\text { Survivors }\end{array}$ & $\begin{array}{l}\text { For } \\
\text { Providers }\end{array}$ & Description \\
\hline Save My Fertility & https://www.savemyfertility.org/ & Yes & No & $\begin{array}{l}\text { An online fertility preservation toolkit for } \\
\text { adult cancer patients to learn more on } \\
\text { preserving fertility before and during } \\
\text { cancer treatment. }\end{array}$ \\
\hline $\begin{array}{l}\text { St. Jude Children's Research } \\
\text { Hospital: Childhood Cancer } \\
\text { Survivor Study Tools and } \\
\text { Documents }\end{array}$ & $\begin{array}{l}\text { https://ccss.stjude.org/tools-and- } \\
\underline{\text { documents.html }}\end{array}$ & No & Yes & $\begin{array}{l}\text { Tools and documents for the St. Jude } \\
\text { Children's Research Hospital's } \\
\text { Childhood Cancer Survivor Study. }\end{array}$ \\
\hline $\begin{array}{l}\text { St. Jude Children's Research } \\
\text { Hospital: Cure4Kids }\end{array}$ & https://www.cure4kids.org/ & No & Yes & $\begin{array}{l}\text { An online resource for healthcare } \\
\text { professionals to enhance the care for } \\
\text { children with cancer. }\end{array}$ \\
\hline $\begin{array}{l}\text { St. Jude Children's Research } \\
\text { Hospital: Together }\end{array}$ & $\begin{array}{l}\text { https://together.stjude.org/en-us/life-after- } \\
\underline{\text { cancer.html }}\end{array}$ & Yes & No & $\begin{array}{l}\text { Offers resources for childhood cancer } \\
\text { survivors on late effects, survivorship } \\
\text { care plans, etc. }\end{array}$ \\
\hline Stupid Cancer®\| & $\begin{array}{l}\text { https://stupidcancer.org/get- } \\
\underline{\text { help/resources/ }}\end{array}$ & Yes & No & $\begin{array}{l}\text { Provides links to resources for } \\
\text { adolescent and young adults with cancer } \\
\text { to find resources and help on topics such } \\
\text { as college and career, mental health, } \\
\text { caregiving, and finances. }\end{array}$ \\
\hline $\begin{array}{l}\text { University of Pennsylvania: } \\
\text { OncoLink }\end{array}$ & https://www.oncolink.org/ & Yes & Yes & $\begin{array}{l}\text { Provides resources and tools for } \\
\text { oncology healthcare professionals, as } \\
\text { well as information for patients on } \\
\text { cancer, diagnosis, treatment, coping, and } \\
\text { survivorship. }\end{array}$ \\
\hline $\begin{array}{l}\text { University of Texas: MD Anderson } \\
\text { Cancer Center Continuing } \\
\text { Medical Education }\end{array}$ & $\begin{array}{l}\text { https://www.mdanderson.org/education- } \\
\underline{\text { training/professional-education/cme- }} \\
\underline{\text { conference-management.html }}\end{array}$ & No & Yes & $\begin{array}{l}\text { Source of information on Continuing } \\
\text { Professional Education/Conference } \\
\text { Management conferences, e-learning } \\
\text { activities, and external educational } \\
\text { partners }\end{array}$ \\
\hline UptoDate & $\begin{array}{l}\text { https://www.uptodate.com/contents/table- } \\
\text { of-contents/oncology/cancer-survivorship }\end{array}$ & No & Yes & $\begin{array}{l}\text { A source of reference with links to most } \\
\text { up to date evidence and clinical guidance } \\
\text { on cancer survivorship related topics. }\end{array}$ \\
\hline $\begin{array}{l}\text { Individual guidelines/reports } \\
\text { Institute of Medicine report: } \\
\text { Childhood Cancer Survivorship: } \\
\text { Improving Care and Quality of Life }\end{array}$ & $\begin{array}{l}\text { Hewitt M, Weiner SL, Simone JV. } \\
\text { Childhood Cancer Survivorship: Improving } \\
\text { Care and Quality of Life. Washington } \\
\text { (DC): National Academies Press (US); } \\
2003\end{array}$ & No & Yes & $\begin{array}{l}\text { Guideline outlining a comprehensive } \\
\text { policy agenda linking improved health } \\
\text { care delivery, investments in education } \\
\text { and training, and expanded research to } \\
\text { improve the long-term outlook for } \\
\text { survivors of childhood cancer. }\end{array}$ \\
\hline
\end{tabular}




\begin{tabular}{|c|c|c|c|c|}
\hline Resource & Link & $\begin{array}{l}\text { For Cancer } \\
\text { Survivors }\end{array}$ & $\begin{array}{l}\text { For } \\
\text { Providers }\end{array}$ & Description \\
\hline $\begin{array}{l}\text { Individual guidelines/reports } \\
\text { Institute of Medicine report: } \\
\text { Ensuring Quality Cancer Care }\end{array}$ & $\begin{array}{l}\text { Hewitt M, Simone JV. Ensuring Quality } \\
\text { Cancer Care. Washington DC. National } \\
\text { Academy Press.1999 }\end{array}$ & No & Yes & $\begin{array}{l}\text { A status report on current health services } \\
\text { (circa 1999) and issues related to } \\
\text { medical coverage, social and economic } \\
\text { status, patient beliefs, physician } \\
\text { decision-making, and other factors. }\end{array}$ \\
\hline $\begin{array}{l}\text { Individual guidelines/reports } \\
\text { Institute of Medicine report: From } \\
\text { Cancer Patient to Cancer } \\
\text { Survivor: Lost in Transition }\end{array}$ & $\begin{array}{l}\text { Hewitt M, Greenfield S, Stovall E. From } \\
\text { Cancer Patient to Cancer Survivor: Lost in } \\
\text { Transition. Washington DC. National } \\
\text { Academy Press.2006 }\end{array}$ & No & Yes & $\begin{array}{l}\text { A book focusing on survivors of adult } \\
\text { cancer during followup phase post } \\
\text { primary treatment. }\end{array}$ \\
\hline $\begin{array}{l}\text { Individual guidelines/reports } \\
\text { President's Cancer Panel Annual } \\
\text { Report 2003-2004, Living Beyond } \\
\text { Cancer: Finding a New Balance }\end{array}$ & $\begin{array}{l}\text { https://deainfo.nci.nih.gov/advisory/pcp/an } \\
\text { nualReports/pcp03-04rpt/Survivorship.pdf }\end{array}$ & No & Yes & $\begin{array}{l}\text { Recommendations for cancer } \\
\text { survivorship compiled from meetings of } \\
\text { the President's Cancer Panel involving } \\
\text { stakeholders such as survivors, } \\
\text { caregivers, advocates, providers, } \\
\text { insurers, Federal and State government } \\
\text { employees, media representatives, and } \\
\text { support organization representatives. }\end{array}$ \\
\hline $\begin{array}{l}\text { Individual guidelines/reports } \\
\text { Shepherd et al. (2010) }\end{array}$ & $\begin{array}{l}\text { Shepherd EJ, Woodgate RL. Cancer } \\
\text { survivorship in children and young adults: } \\
\text { a concept analysis. J Pediatr Oncol Nurs. } \\
2010 \text { Mar-Apr;27(2):109-18. Doi: } \\
\text { 10.1177/1043454209349807. PMID: } \\
20044589 .\end{array}$ & No & Yes & $\begin{array}{l}\text { An article providing a conceptual } \\
\text { framework helping to define survivorship } \\
\text { and useful for research and clinical } \\
\text { practice in pediatric oncology. }\end{array}$ \\
\hline $\begin{array}{l}\text { Individual guidelines/reports } \\
\text { NCCN: Guidelines for Patients }\end{array}$ & $\begin{array}{l}\text { https://www.nccn.org/patients/guidelines/c } \\
\text { ancers.aspx }\end{array}$ & Yes & No & $\begin{array}{l}\text { National Comprehensive Cancer } \\
\text { Network's guidelines for cancer patients. }\end{array}$ \\
\hline $\begin{array}{l}\text { Individual guidelines/reports } \\
\text { NCCN: Clinical Practice } \\
\text { Guidelines }\end{array}$ & $\begin{array}{l}\text { https://www.nccn.org/patients/clinical/defa } \\
\text { ult.aspx }\end{array}$ & No & Yes & $\begin{array}{l}\text { National Comprehensive Cancer } \\
\text { Network's guidelines for clinicians and } \\
\text { health professionals on cancer } \\
\text { treatments. }\end{array}$ \\
\hline $\begin{array}{l}\text { Individual guidelines/reports } \\
\text { COG: Survivorship Guidelines }\end{array}$ & $\begin{array}{l}\text { https://childrensoncologygroup.org/index.p } \\
\text { hp/survivorshipguidelines }\end{array}$ & No & Yes & $\begin{array}{l}\text { Cancer survivorship guidelines for } \\
\text { clinicians who are providing ongoing } \\
\text { healthcare to survivors. }\end{array}$ \\
\hline $\begin{array}{l}\text { Individual guidelines/reports } \\
\text { ASCO: Patient and Survivor Care }\end{array}$ & $\begin{array}{l}\text { https://www.asco.org/research- } \\
\text { guidelines/quality- } \\
\text { guidelines/guidelines/patient-and-survivor- } \\
\text { care }\end{array}$ & No & Yes & $\begin{array}{l}\text { Source of clinical guidelines on cancer } \\
\text { treatment and survivor care. }\end{array}$ \\
\hline
\end{tabular}




\begin{tabular}{|c|c|c|c|c|}
\hline Resource & Link & $\begin{array}{l}\text { For Cancer } \\
\text { Survivors }\end{array}$ & $\begin{array}{l}\text { For } \\
\text { Providers }\end{array}$ & Description \\
\hline $\begin{array}{l}\text { International } \\
\text { Dutch Childhood Oncology Group }\end{array}$ & https://www.dcog-ectc.nl/ & No & Yes* $^{*}$ & $\begin{array}{l}\text { The Dutch Childhood Oncology Group - } \\
\text { Early Clinical Trial Consortium (DCOG- } \\
\text { ECTC) implements clinical trials in } \\
\text { children with cancer. The website } \\
\text { provides tools for investigators in } \\
\text { participating DCOG-ECTC studies. }\end{array}$ \\
\hline $\begin{array}{l}\text { International } \\
\text { International Guideline } \\
\text { Harmonization Group }\end{array}$ & www.ighg.org & No & Yes & $\begin{array}{l}\text { An organization that develops } \\
\text { international guidelines for the long-term } \\
\text { followup of childhood, adolescent, and } \\
\text { young adult cancer survivors. }\end{array}$ \\
\hline $\begin{array}{l}\text { International } \\
\text { PanCareSurFup }\end{array}$ & https://www.pancaresurfup.eu/ & No & Yes & $\begin{array}{l}\text { An organization consisting of } 16 \\
\text { European institutions establishing } \\
\text { guidelines for followup; provide training } \\
\text { and workshops to stakeholders. }\end{array}$ \\
\hline $\begin{array}{l}\text { International } \\
\text { Scottish Intercollegiate Guidelines } \\
\text { Network }\end{array}$ & https://www.sign.ac.uk/our-guidelines/ & No & Yes & $\begin{array}{l}\text { Healthcare group to improve the quality } \\
\text { of healthcare for patients in Scotland } \\
\text { through development and dissemination } \\
\text { of clinical guidelines. }\end{array}$ \\
\hline $\begin{array}{l}\text { International } \\
\text { Union for International Cancer } \\
\text { Control: Sustainable Development } \\
\text { Goals }\end{array}$ & $\begin{array}{l}\text { https://www.uicc.org/search/site/?f\%5B0\% } \\
\text { 5D=sm index page type } \% 3 \text { AResources }\end{array}$ & No & Yes & $\begin{array}{l}\text { Union for International Cancer Control } \\
\text { (UICC) represents over } 1200 \\
\text { organizations including major cancer } \\
\text { societies, ministries of health and patient } \\
\text { groups, providing an avenue for } \\
\text { advocacy, guidelines, and policy } \\
\text { development. }\end{array}$ \\
\hline $\begin{array}{l}\text { International } \\
\text { United Kingdom Children's } \\
\text { Cancer and Leukaemia Group }\end{array}$ & https://www.cclg.org.uk/ & Yes & Yes & $\begin{array}{l}\text { A professional association providing } \\
\text { advice and information to government } \\
\text { agencies, and childhood cancer } \\
\text { survivors on childhood cancer, as well as } \\
\text { funding research. }\end{array}$ \\
\hline
\end{tabular}

CAHPS $=$ Medicare Consumer Assessment of Healthcare Providers and Systems; NCI $=$ National Cancer Institute; NIH $=$ National Institutes of Health; SEER $=$ Surveillance Epidemiology and End Results data 


\section{Appendix F. Program Theory Variable List}

\section{Environment}

- Urban, suburban, rural

- Distance to clinic

- Regional differences (added to refined program theory)

- Crisis events

\section{HealthCare System}

- Degree of fragmentation/integration

- Availability of financial and other resources

- Telemedicine

- Integrated electronic health records

- Use of multidisciplinary teams

- Availability of needed specialists

\section{Provider Characteristics}

- Identity/Specialty (oncology, primary care)

- Years in practice

- Race/language/cultural concordance

- Gender (added to refined program theory)

- Survivor volume (added to refined program theory)

\section{Survivor Characteristics}

- Age at treatment

- Time since diagnosis (longitudinal and calendar time)

- Developmental age/Cognitive function/Educational attainment

- Cancer type (added to refined program theory)/complexity of diagnosis

- Treatment exposures

- Genetics

- Life transitions (e.g., marriage, moving)

- Race

- Gender

- Education (removed from the initial program theory)

- Marital status (added to refined program theory)

- Social determinants of health

- Work status (added to refined program theory)

- Current age (added to refined program theory)

\section{Survivor Needs}

- Presence of chronic medical conditions

- Incidence and severity of late effects, including neurocognitive effects 


\section{Facilitators/Barriers}

\section{Provider}

- Financial and other resources

- Clinic staffing

- Time

○ Reimbursement

○ Volume

- Incentives (e.g., monetary, quality measures)

- Relationship with survivors

- Willingness to transition away (oncology)/accept (PCP) care for cancer survivors

- Knowledge

- About childhood cancer survivors' needs generally

- About a particular survivor's needs (e.g., through a survivorship care plan, access to medical records)

- Awareness of survivorship resources/guidelines

- Comfort treating childhood cancer survivors (relates to knowledge base and professional expertise)

- Communication and care coordination between and among the pediatric cancer center, the primary care provider, and the survivor

\section{Survivor}

- Financial and other resources

- Financial costs (e.g., reimbursement, ability to pay co-pay)

- Access to care

$\circ$ Health insurance

- Time

- Transportation

- Willingness to transition away from cancer providers (or not)

○ Motivation

- Provider influences

- Belief in PCP ability to meet their needs

- Knowledge

- Of their treatment and evolving associated risks

- Of their evolving followup care needs

- Ability to find appropriate care

- Coordination of multiple specialists

- Psychosocial factors

- Fear of recurrence

- Post-traumatic stress disorder

- Anxiety

- Depression

- Cognitive deficit

- Impact of cancer on identity 
- Autonomy/Personal responsibility/Self-reliance/Self-efficacy/Patient activation

- Degree of family/parental support for followup care

- Awareness and availability of (culturally appropriate) resources (added to refined program theory)

\section{Provider Health Practices}

- Oncologist (added to refined program theory): return survivor to informed PCP/help survivors identify knowledgeable providers; $P C P$ : accept survivors into practice and learn about survivorship needs (added to refined program theory)

- Connect survivors with survivorship resources and services

- Conduct guideline-concordant surveillance for long-term and late effects

- Manage symptoms/late effects

- Educate survivors about late effects

- Assess psychosocial needs and provide psychosocial support

- Counsel regarding healthy behaviors

- Coordinate care

- Refer to appropriate specialists (medical, legal, financial)

\section{Survivor Health Behaviors}

- Receive surveillance for long-term and late effects and appropriate preventive care

- Receive social support, nutritional, rehabilitative, and fertility preservation services

- Health behaviors

- Alcohol/Tobacco/Other drugs

- Physical activity

- Loss to followup

- Emergency department visits

- Hospitalizations

\section{Health Status}

- Mortality

- Morbidity/Late effects

- Peripheral neuropathy

- Cardiac dysfunction

- Cognitive problems

- Bone loss

○ Infertility

- Sexual dysfunction

- Insomnia

- Obesity

- Quality of life (health-related)/Functional status

$\circ$ Fatigue

○ Pain

- Vasomotor and menopausal symptoms 
- Psychosocial needs

○ Depression

$\circ$ Anxiety

\section{Consumer Satisfaction}

\section{Costs}

\section{Models Of Survivorship Care}

- 4 dimensions:

- Presence of survivorship expertise (i.e., does at least one provider in the model have particular expertise in survivorship?) [yes/no/unclear]

- Is the survivorship expert trained in oncology, primary care, other, unclear?

- Is the survivorship expertise $\mathrm{MD}, \mathrm{NP} / \mathrm{PA}$, multidisciplinary, unclear?

- Role of PCP [main provider of survivorship care, provides survivorship care under the guidance of survivorship expert, provides primary care with no particular attention to survivorship, unclear]

- Access to academic/cancer center support (for survivors and/or providers (added to refined program theory)) [yes/no/unclear]

- Consultative vs longitudinal or unclear

\section{Resources}

- long-term followup guidelines

- educational materials directed at either survivor/family or care providers regardless of media (i.e., electronic, hard copy)

$\circ \quad$ e.g., website with information and opportunity for Q\&A

○ "health links"

- in-person or virtual trainings (i.e., workshops, conferences, CME courses) directed at either survivor/family or care providers (MDs, NPs, PAs; both current and for students/trainees)

- survivor care documents (i.e., survivor -specific standardized letters, treatment summaries, survivorship care plans)

- survivorship care management processes (i.e., expedited routes of contact for consultation, re-referral, support services; methods for digitizing and securely distributing health records; and other provider-to-provider and provider-to- survivor communications)

- survivor supportive tools and services (i.e., in-person or digital)

$\circ$ text messaging/peer navigator program

- support groups (in-person, telephone-based, or online)

○ professional psychosocial counselors (in-person, telephone based, or online) 


\section{Appendix G. Initial Program Theory}

Figure G-1. Initial program theory

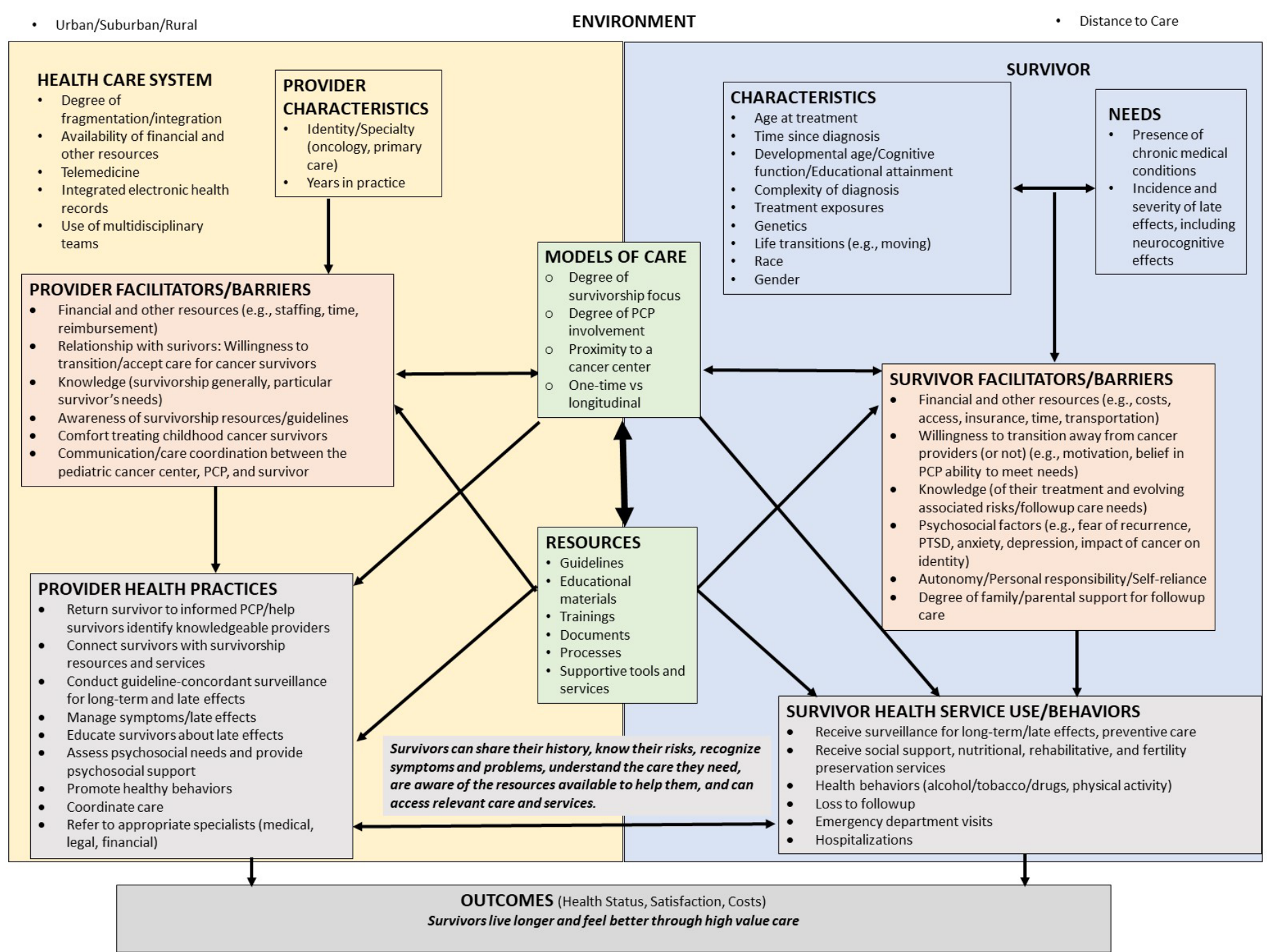

